\title{
Synthesis of 2-(1H-Indol-2-yl)acetamides via Bronsted Acid-Assisted Cyclization Cascade
}

Nicolai A. Aksenov,,$^{\dagger *}$ Dmitrii A. Aksenov ${ }^{\dagger}$ Anton A. Skomorokhov, ${ }^{\dagger}$ Lidiya A. Prityko, ${ }^{\dagger}$ Alexander V. Aksenov, ${ }^{\dagger}$

Georgii D. Griaznov, and Michael Rubin,,$^{\dagger, *^{*}}$

${ }^{\dagger}$ Department of Chemistry, North Caucasus Federal University, 1a Pushkin St., Stavropol 355009, Russia;

${ }^{\ddagger}$ Department of Chemistry, University of Kansas, 1567 Irving Hill Road, Lawrence, KS 66045, USA.

\section{SUPPORTING INFORMATION}

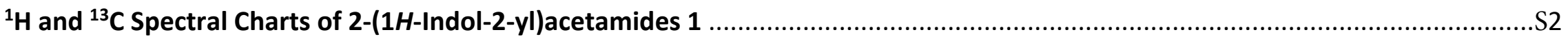

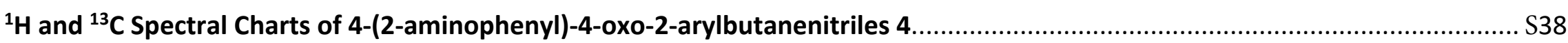

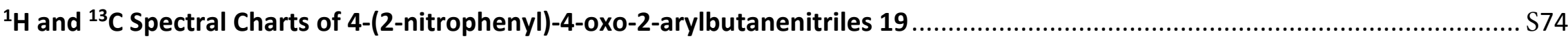

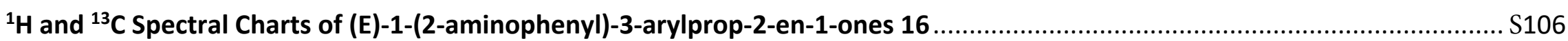

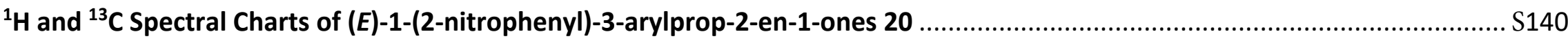

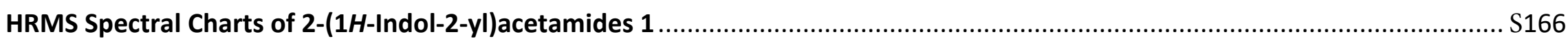

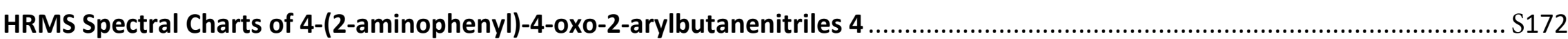

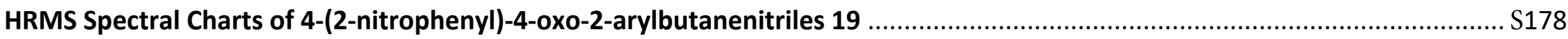

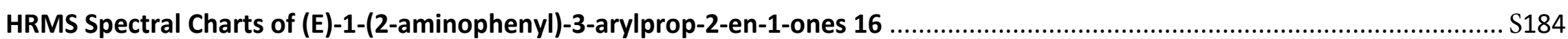

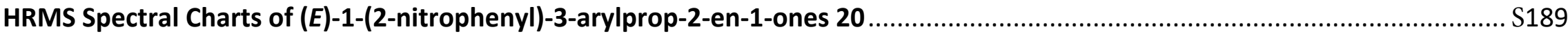

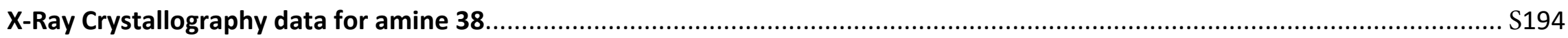


${ }^{1} \mathrm{H}$ and ${ }^{13} \mathrm{C}$ Spectral Charts of 2-(1H-Indol-2-yl)acetamides 1

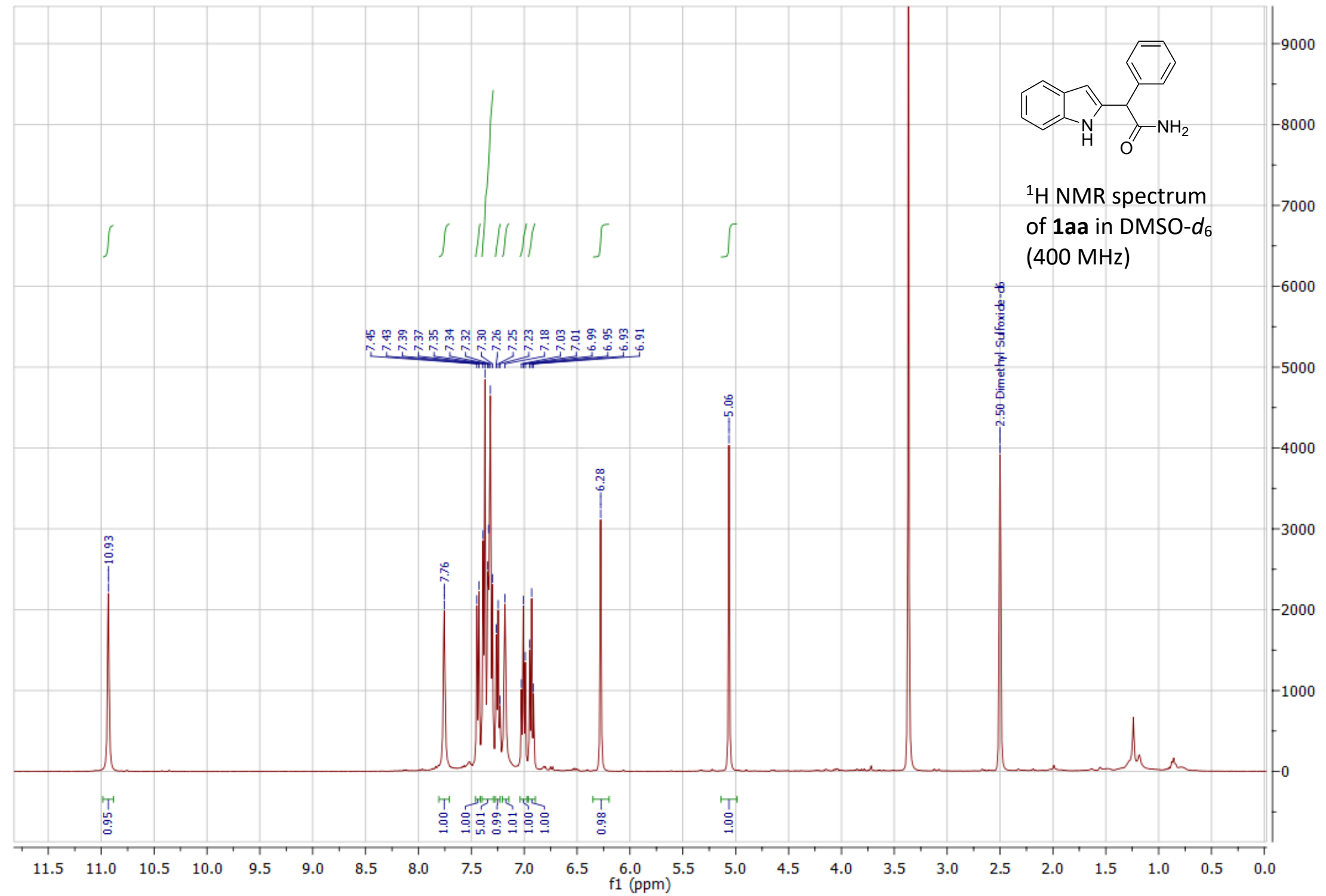




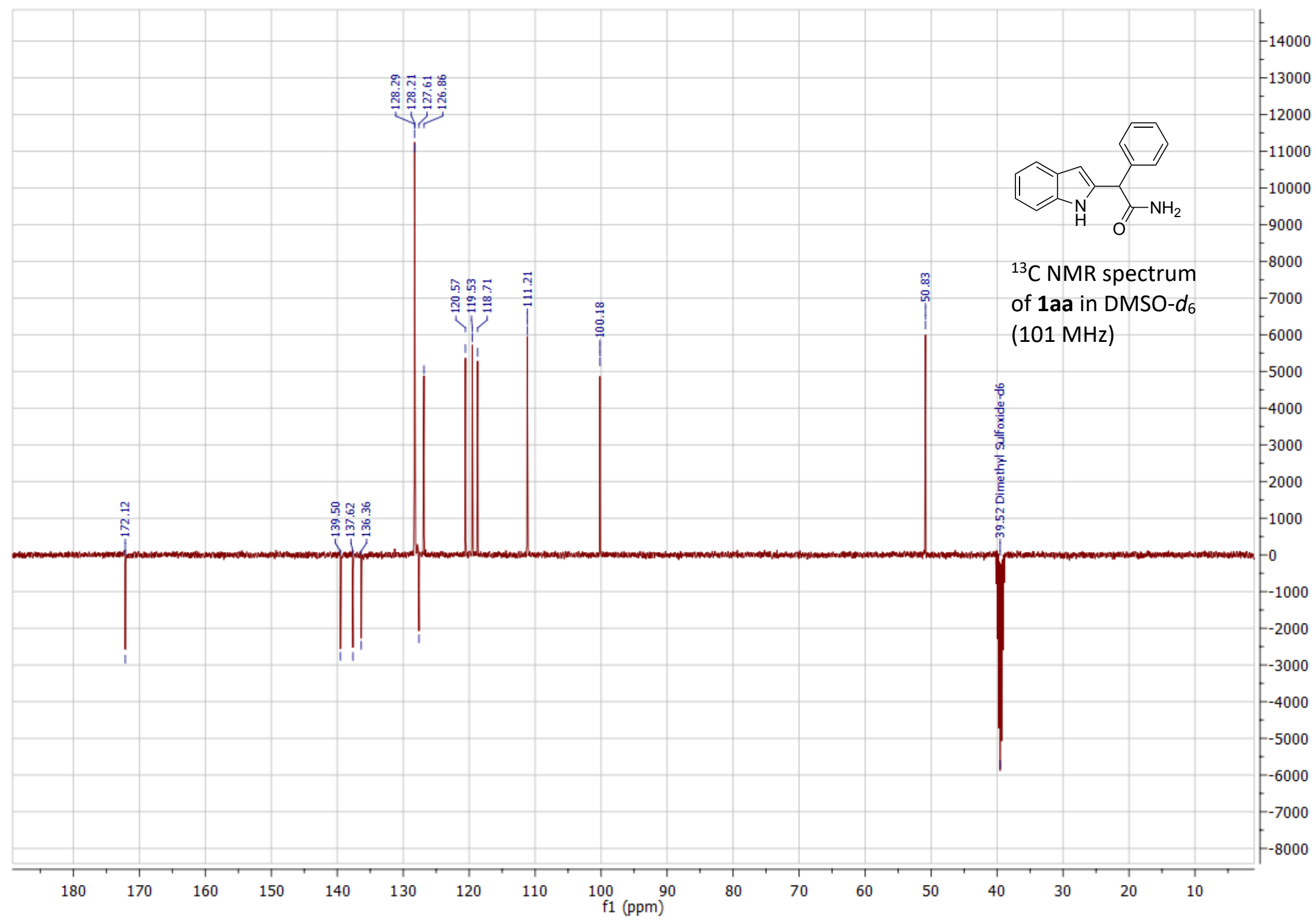




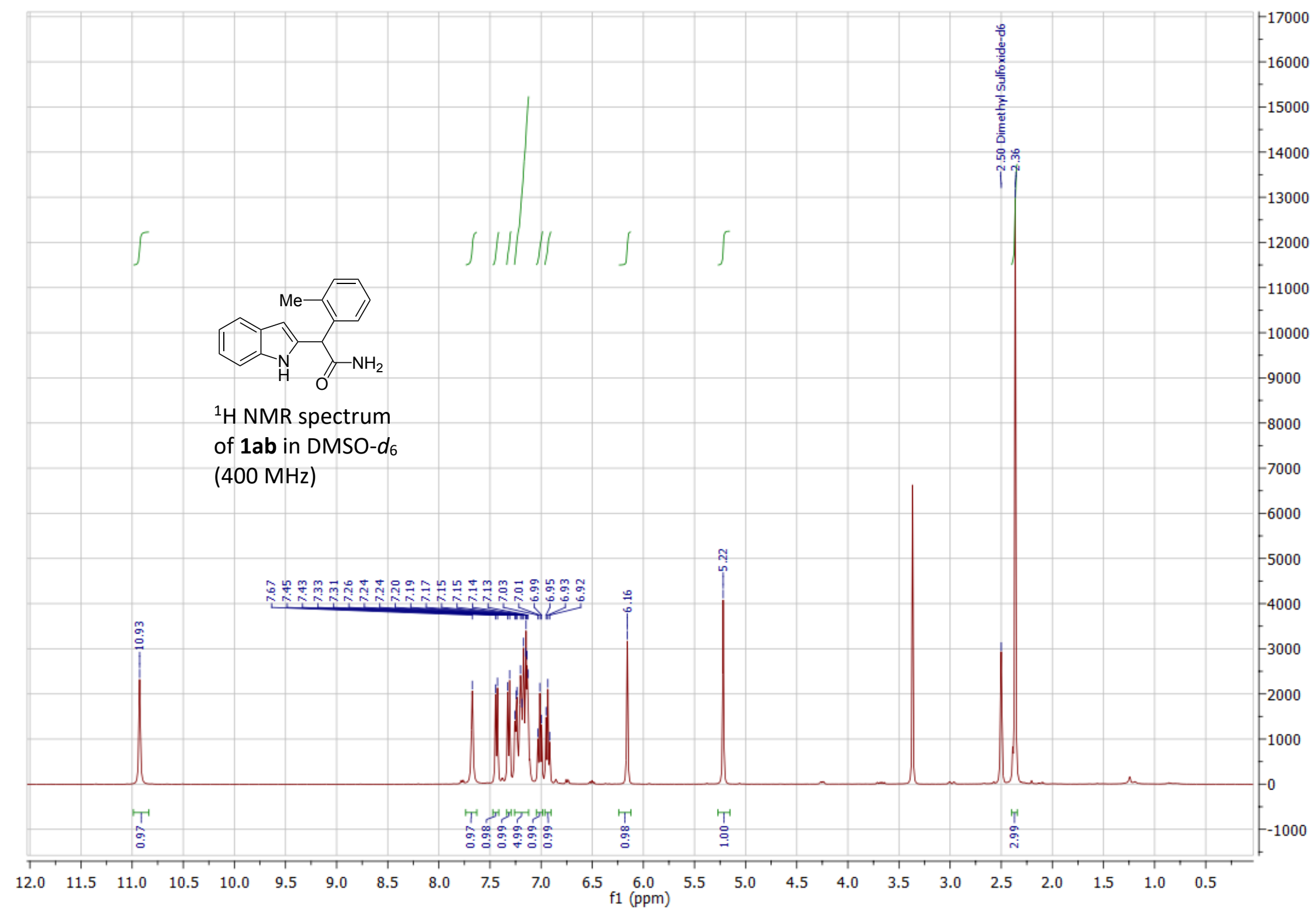




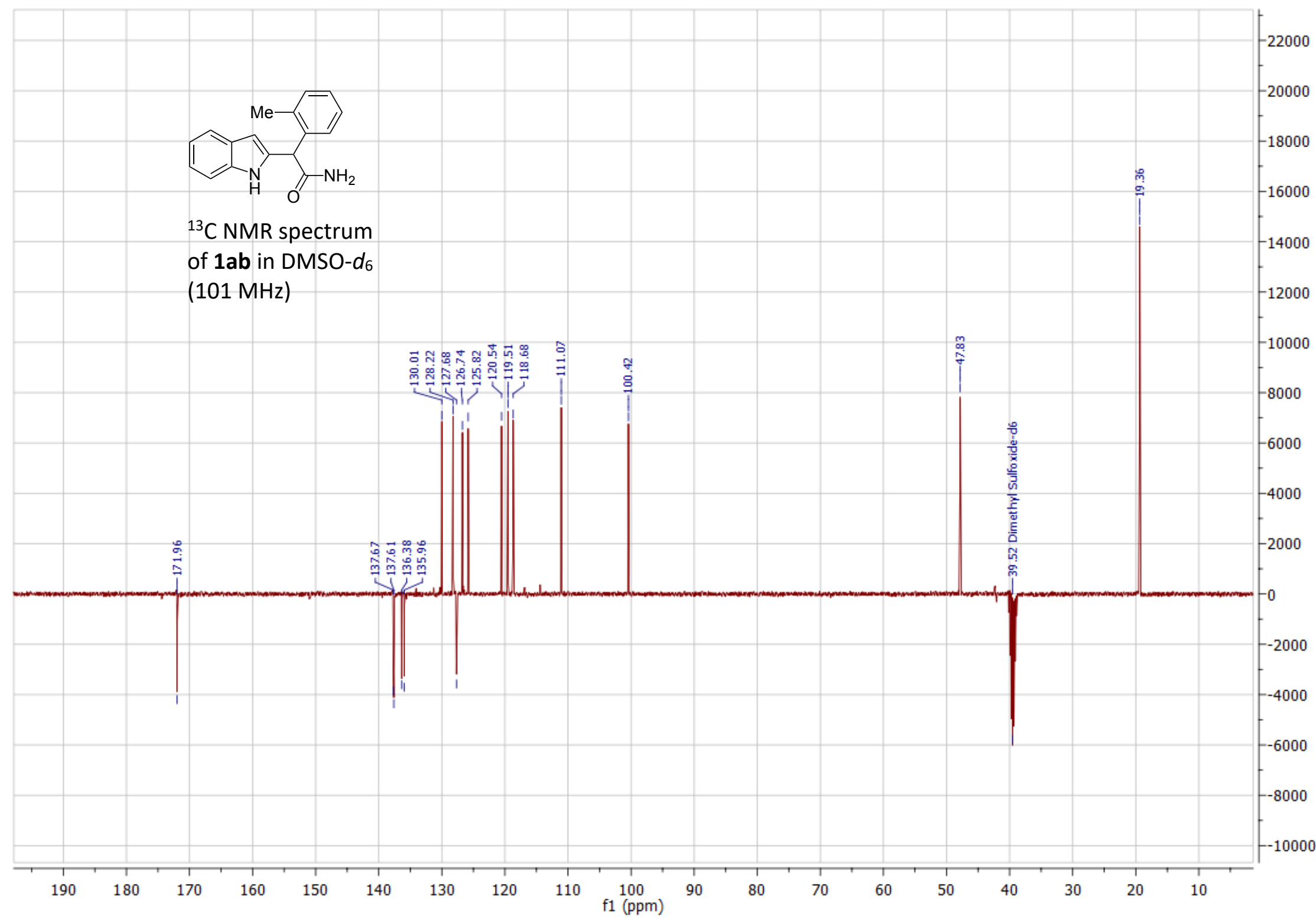




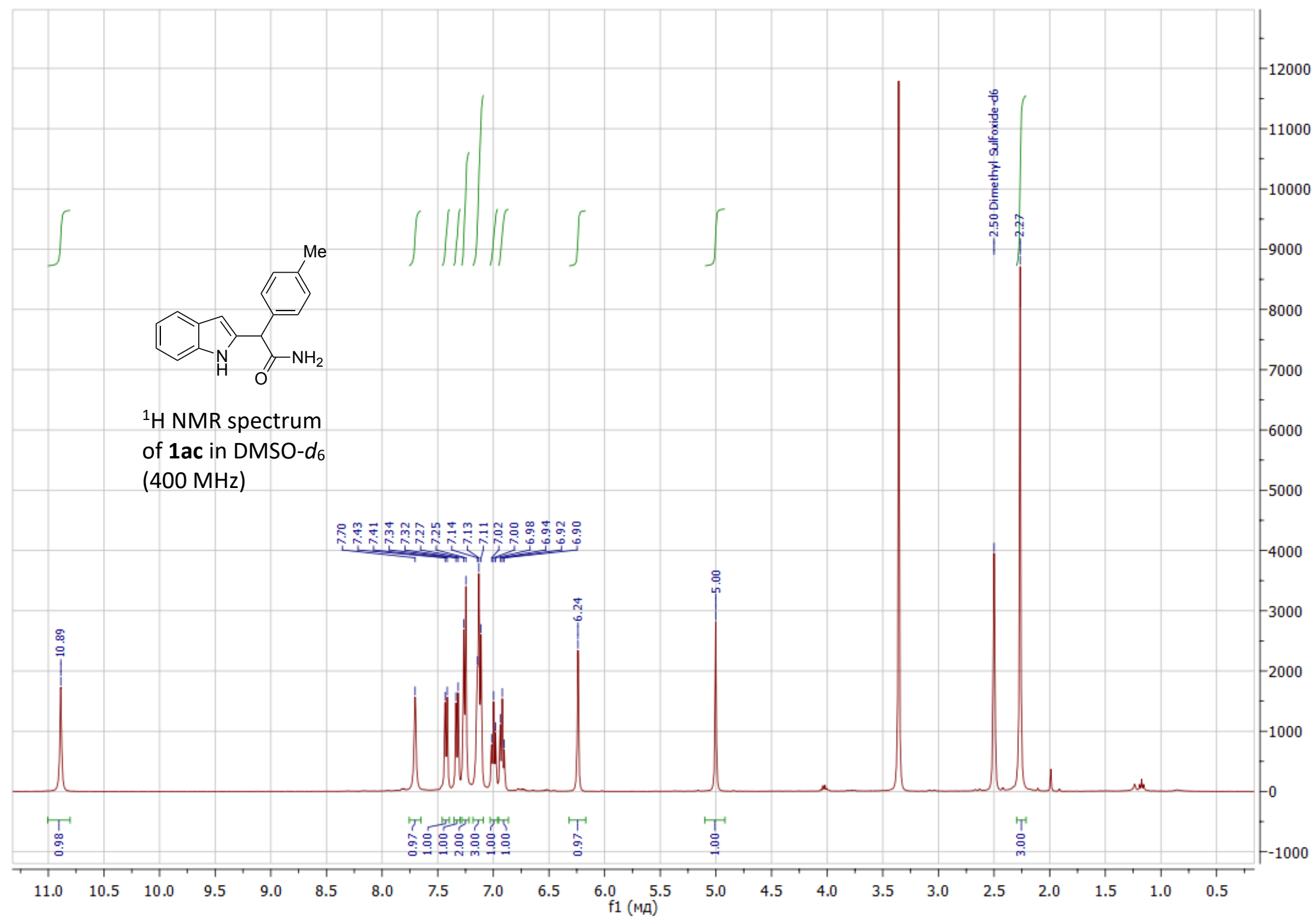




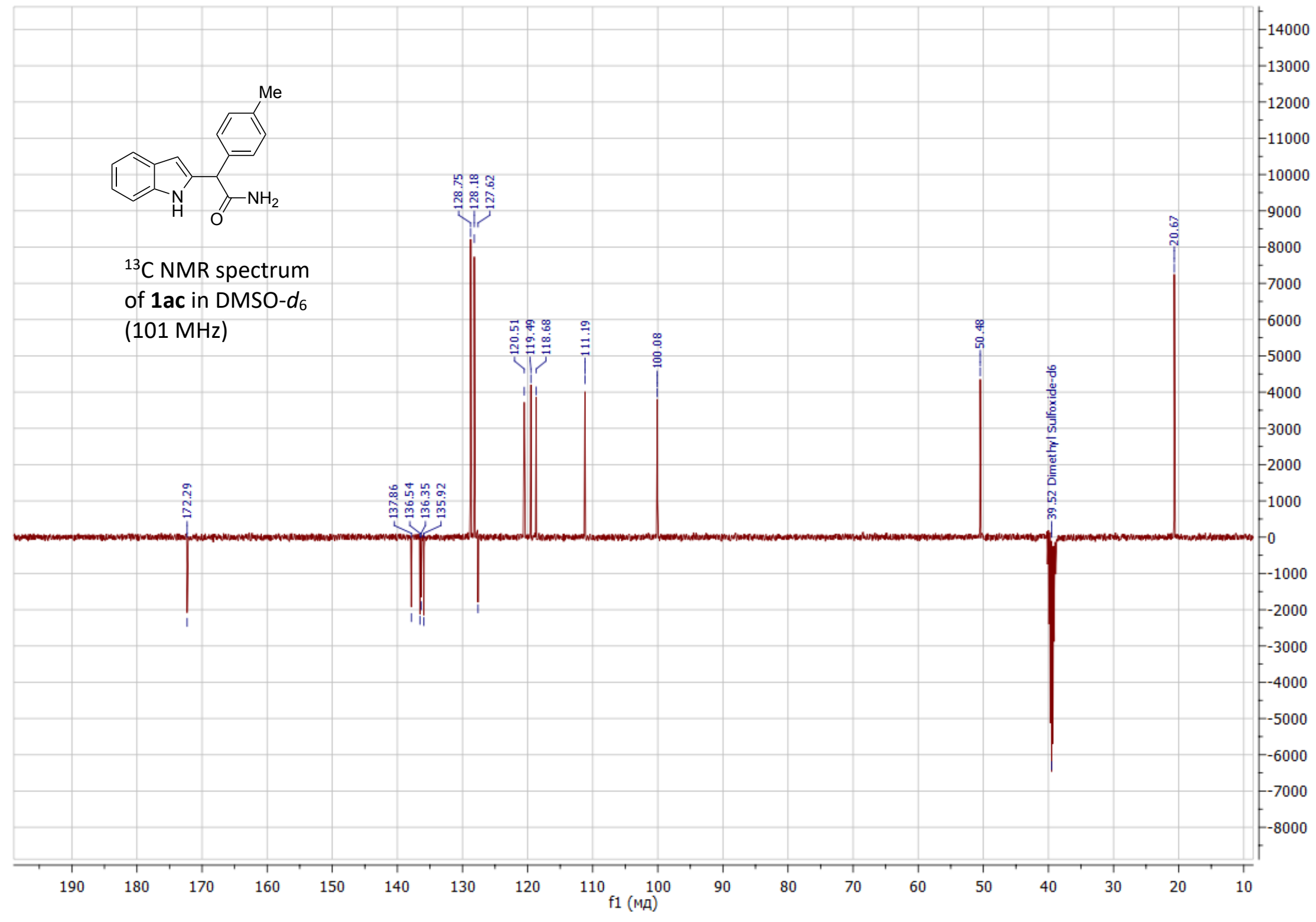




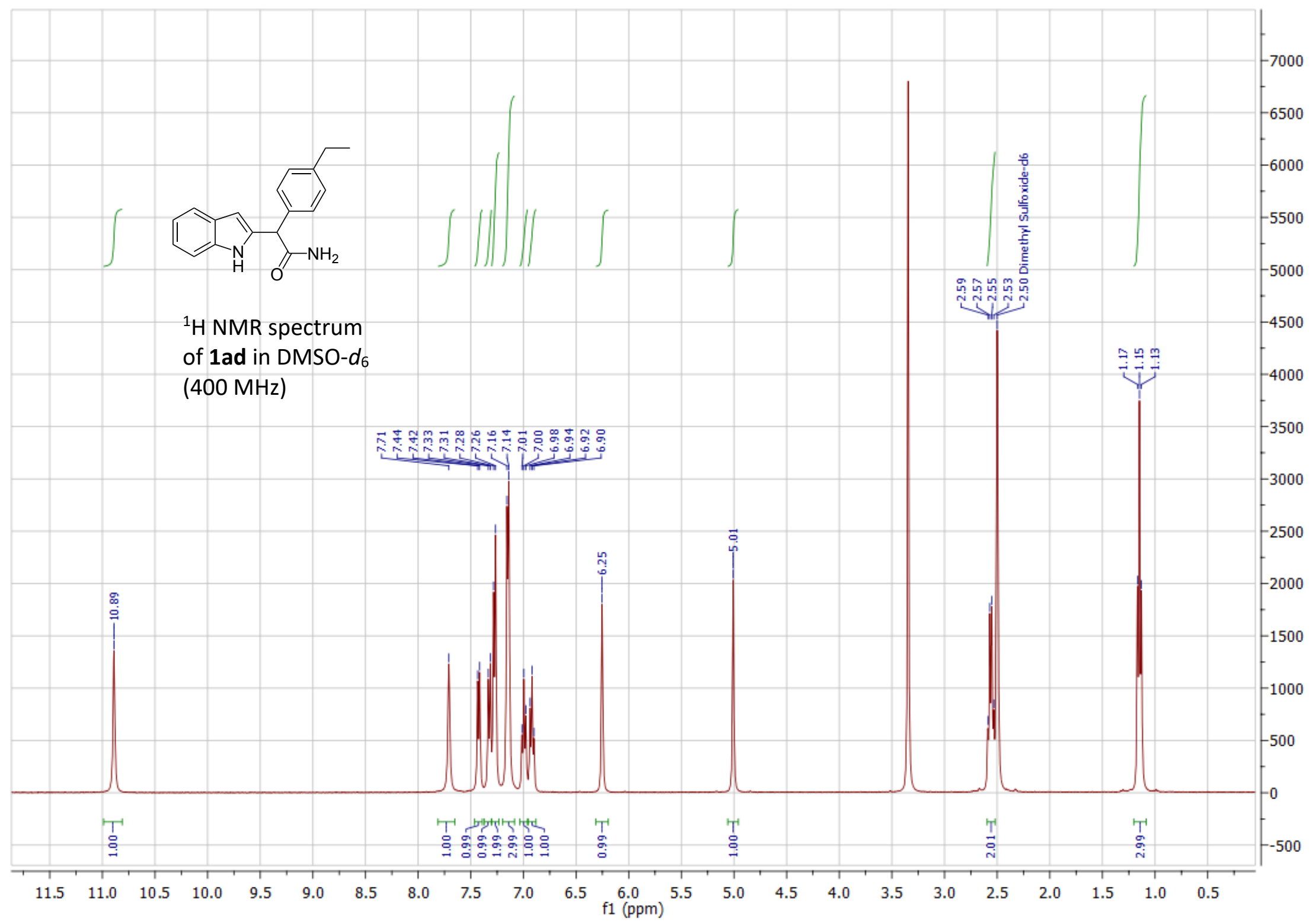




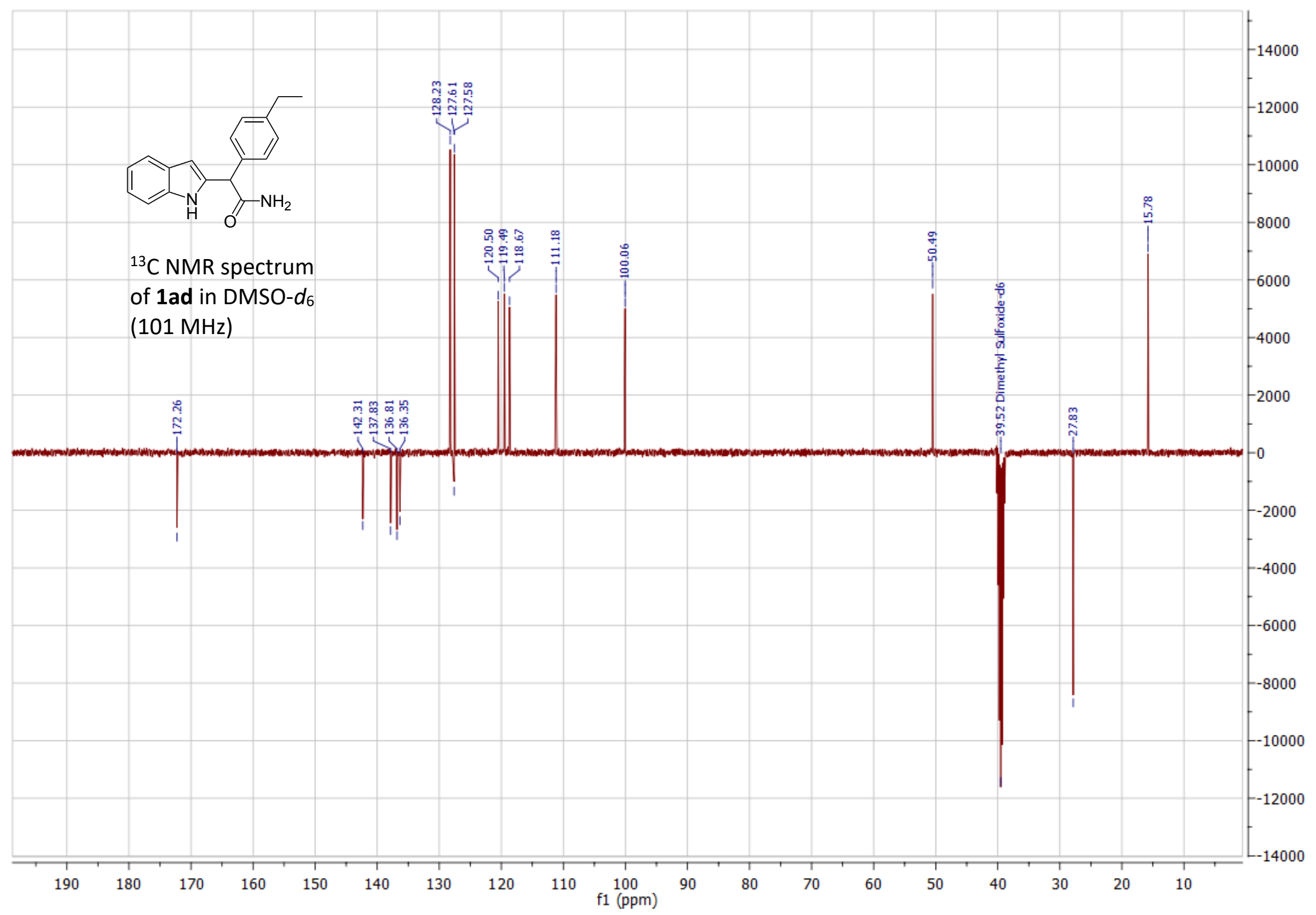




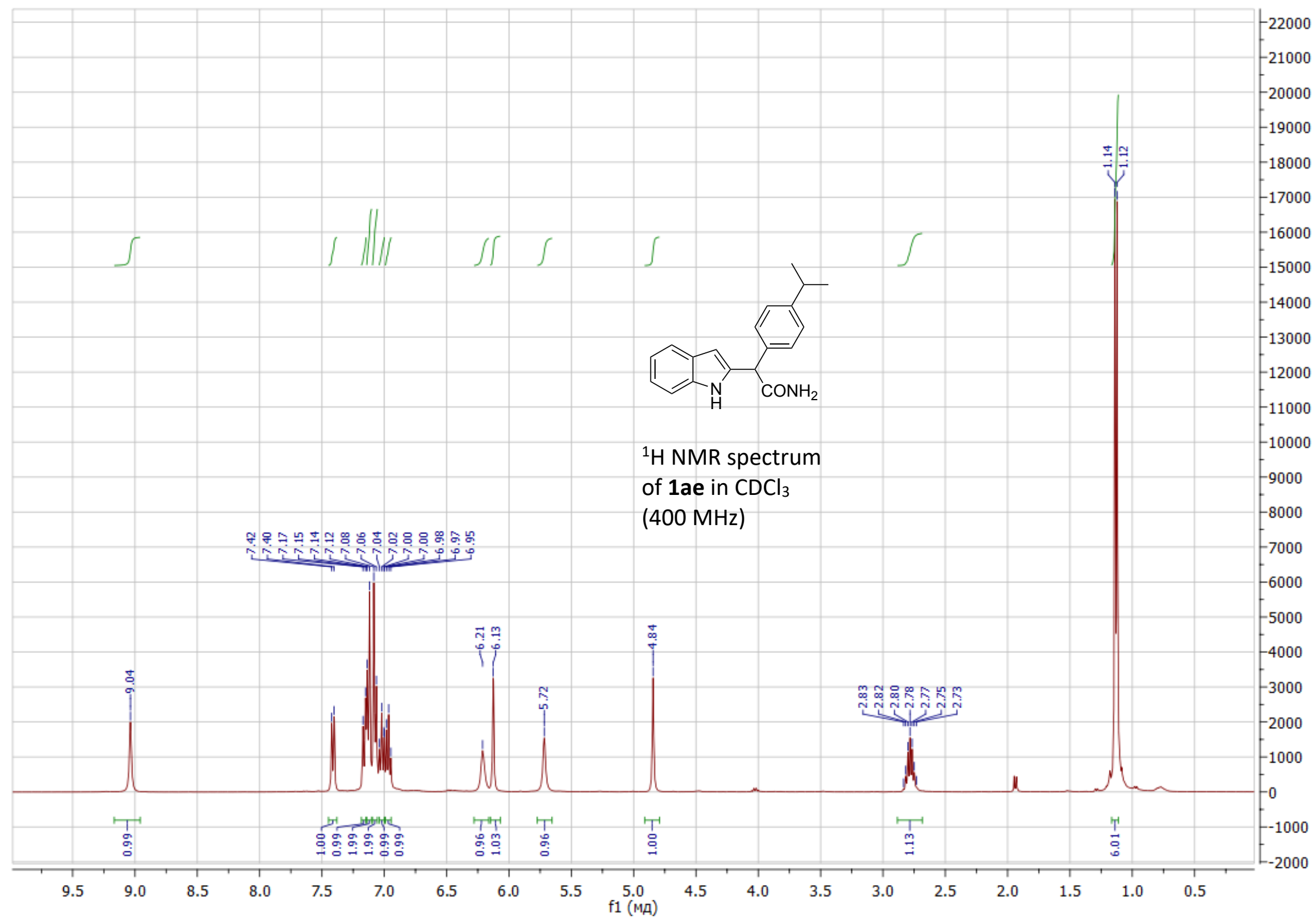




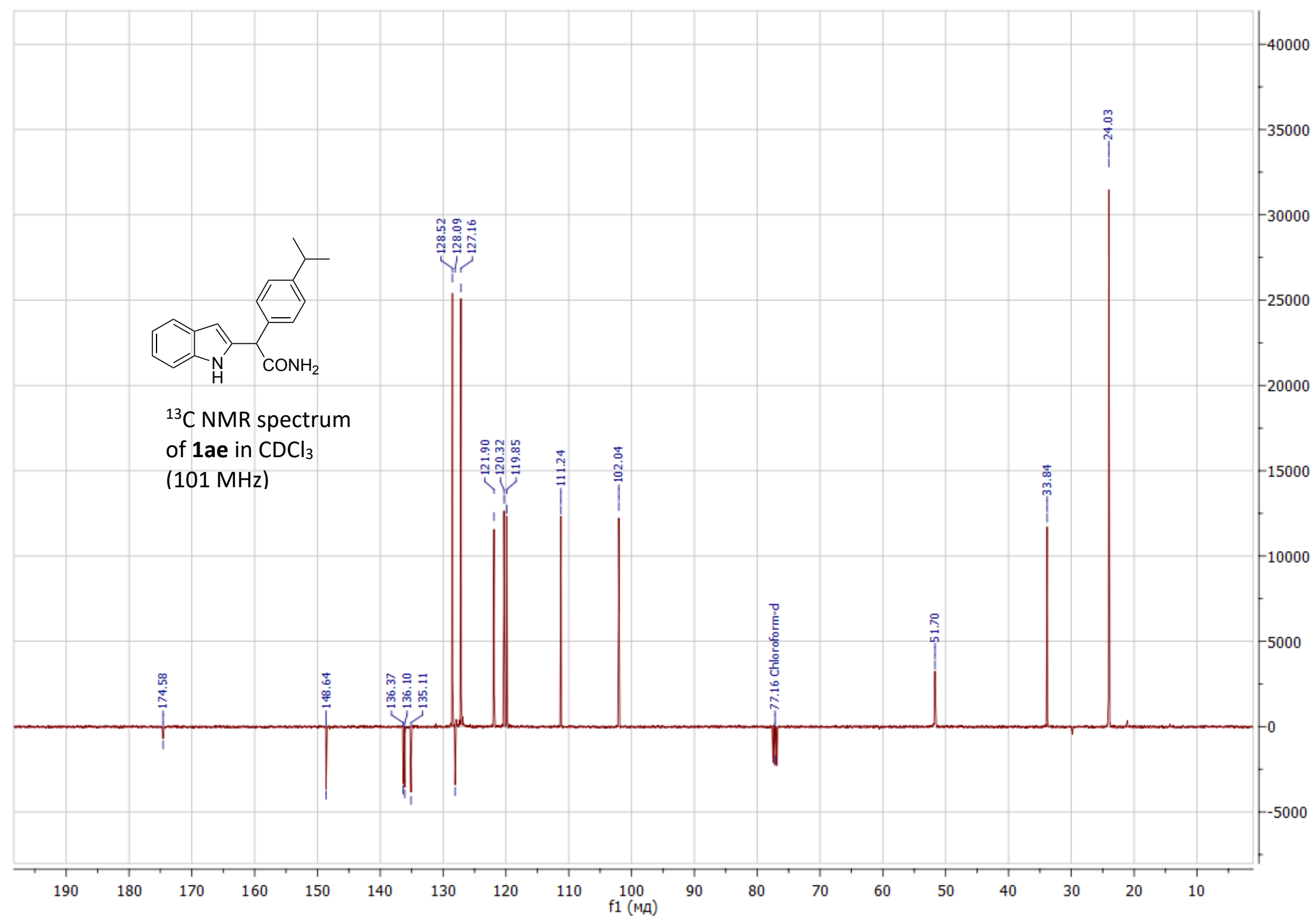




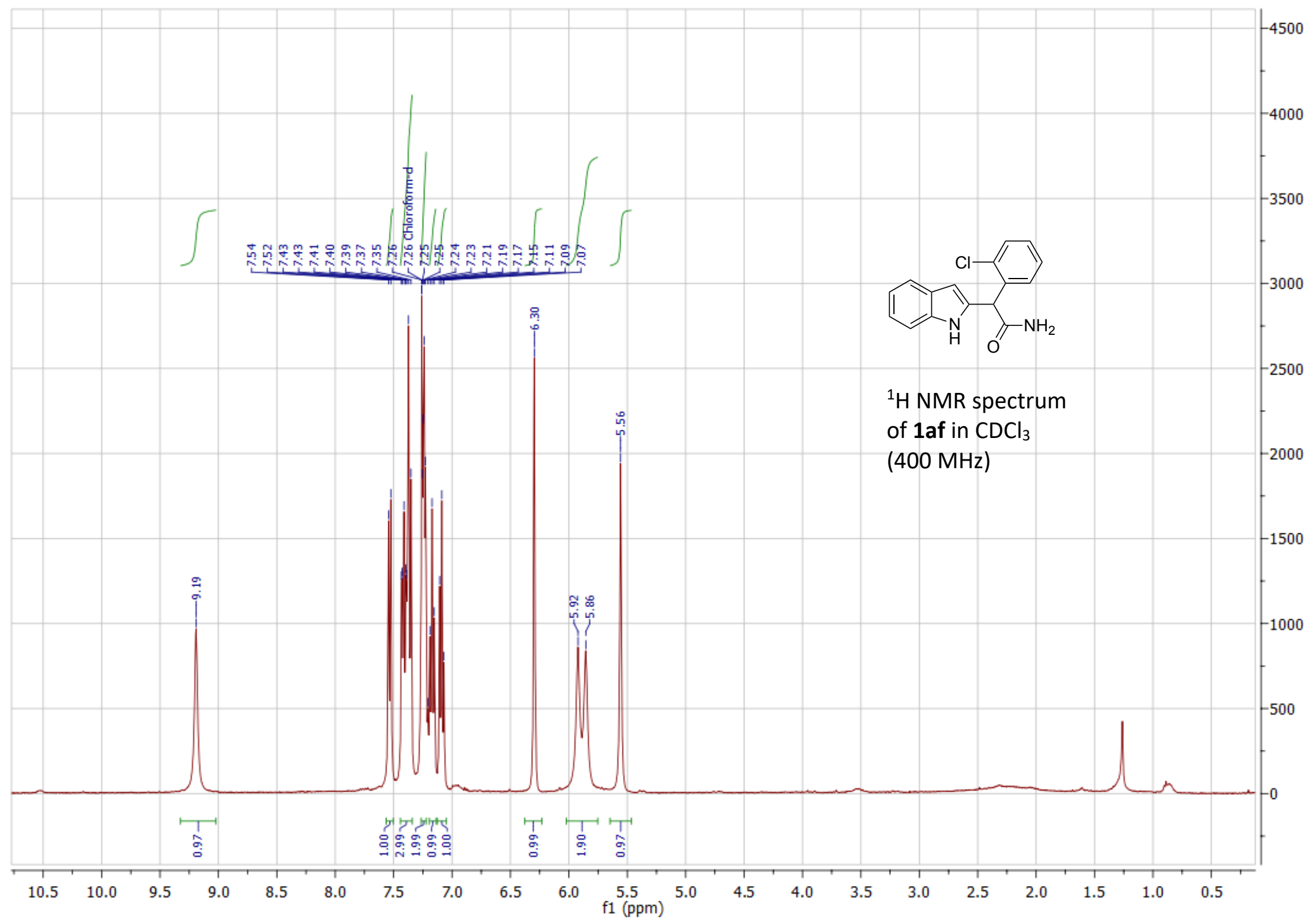




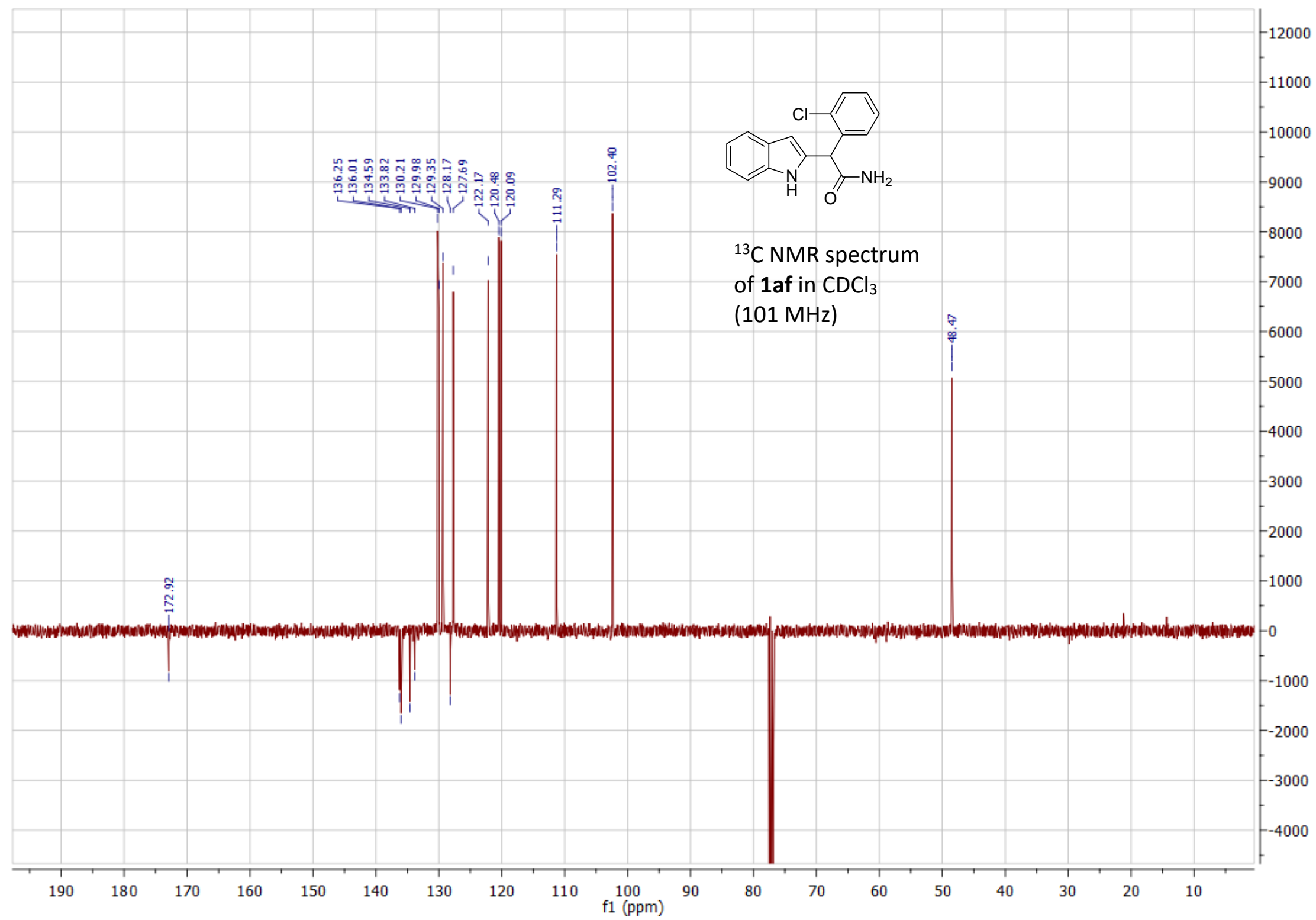




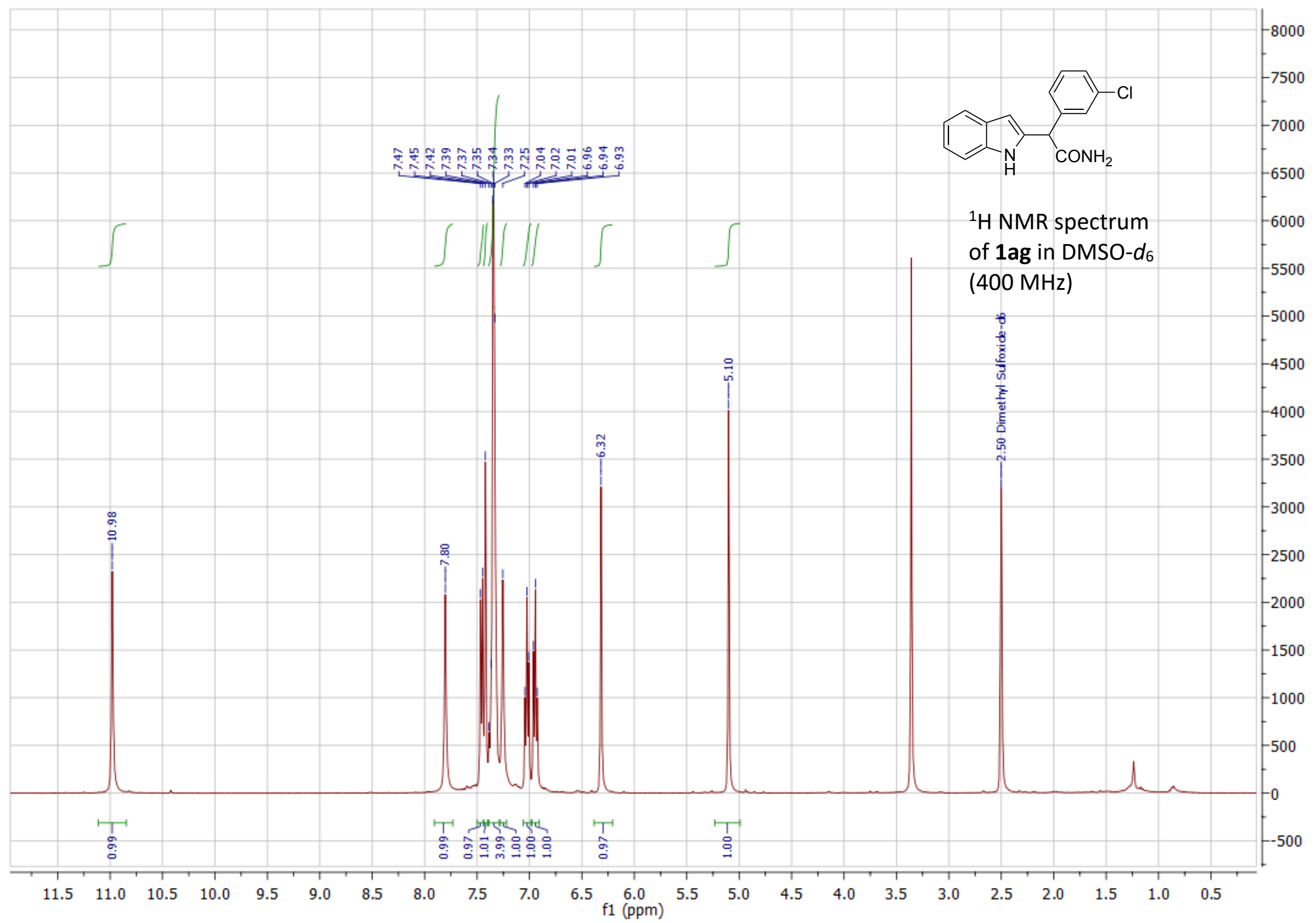




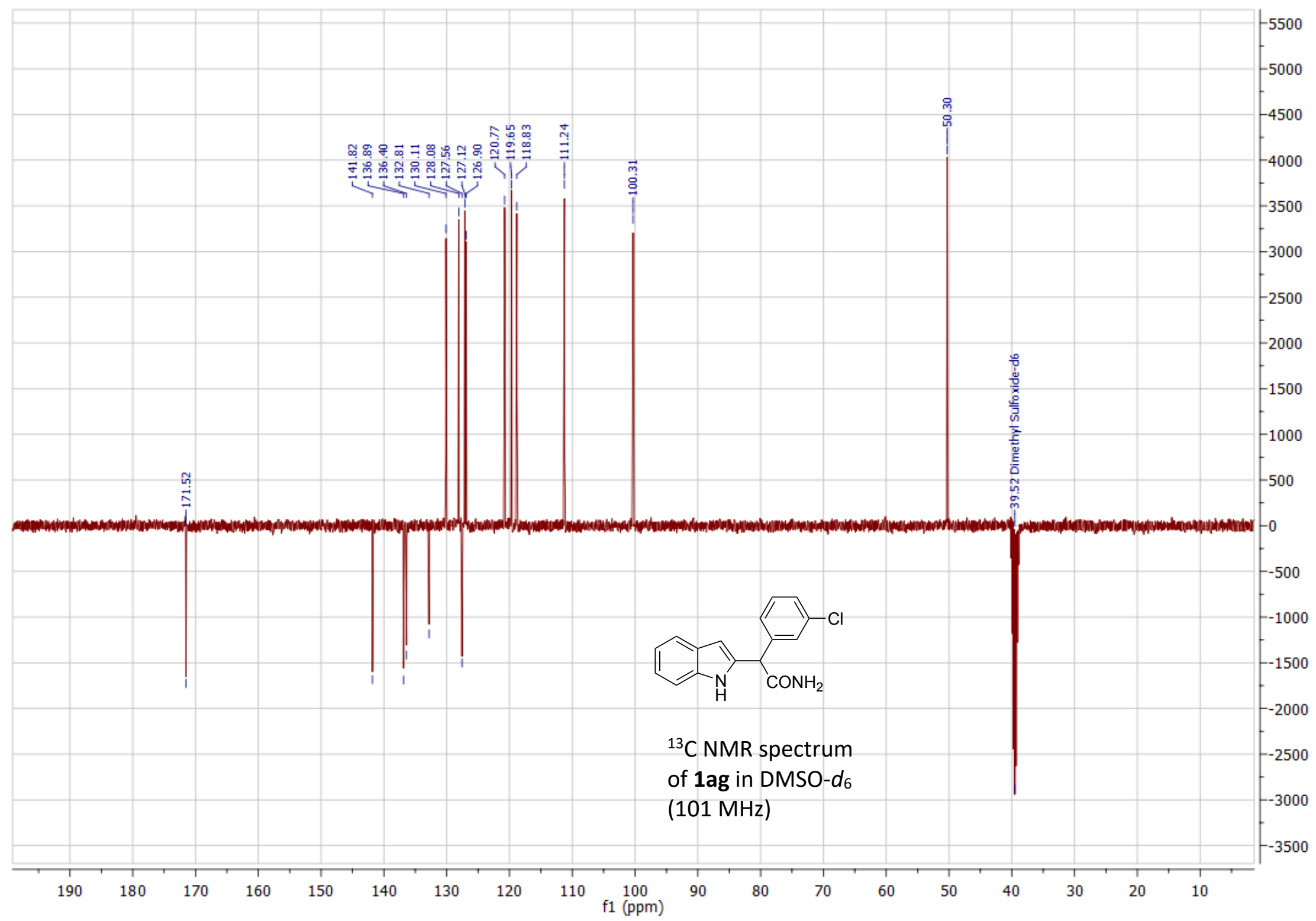




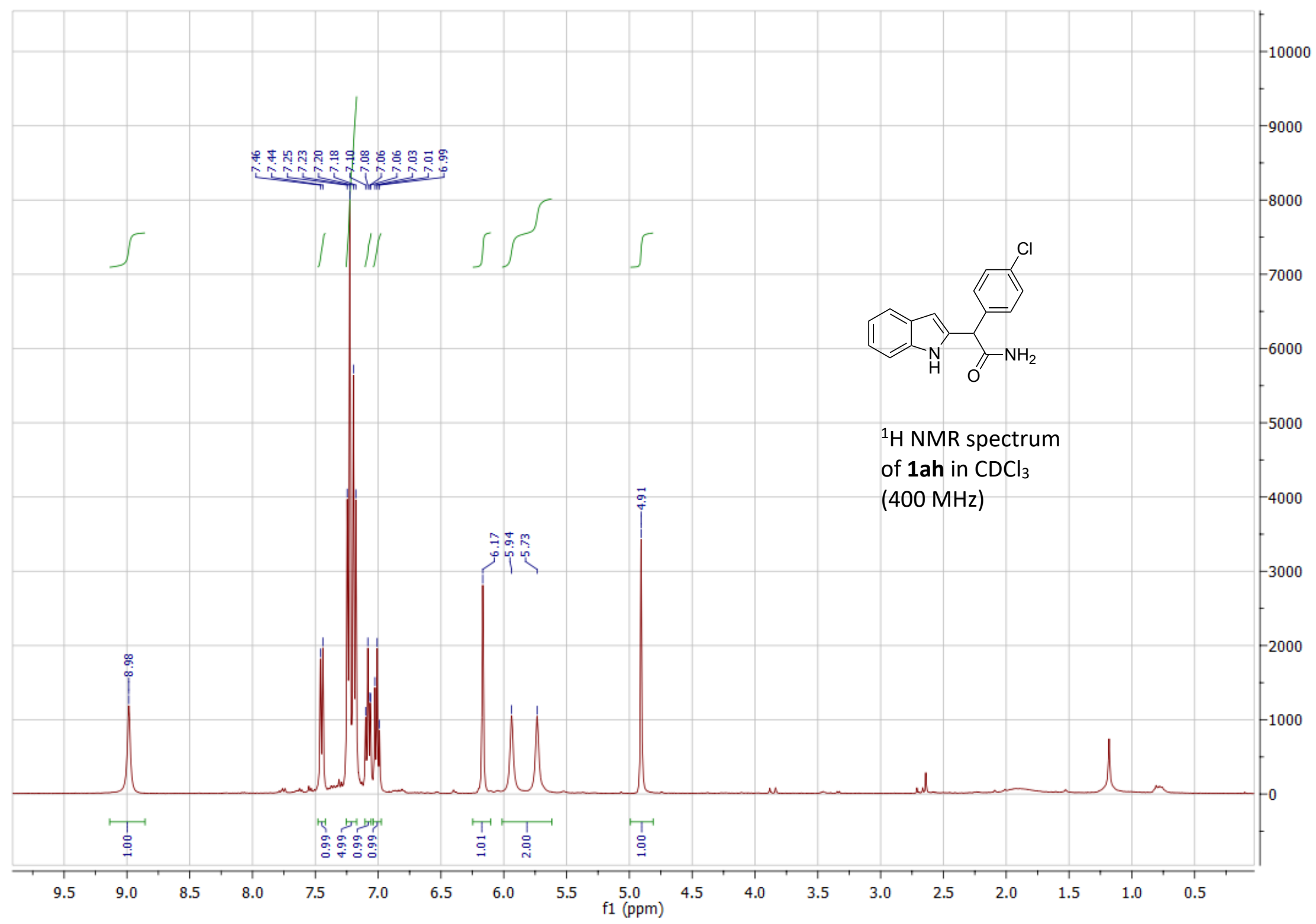




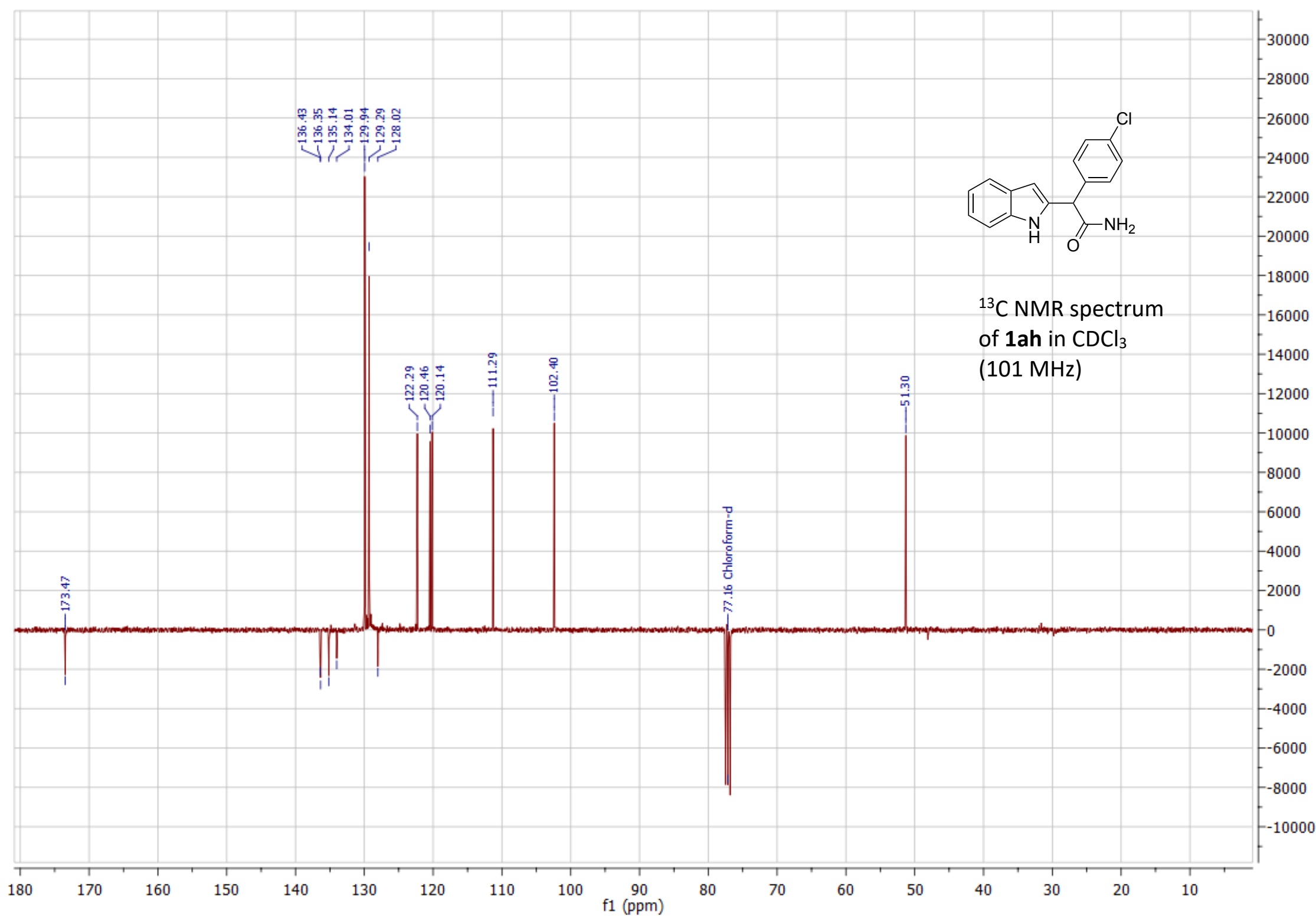




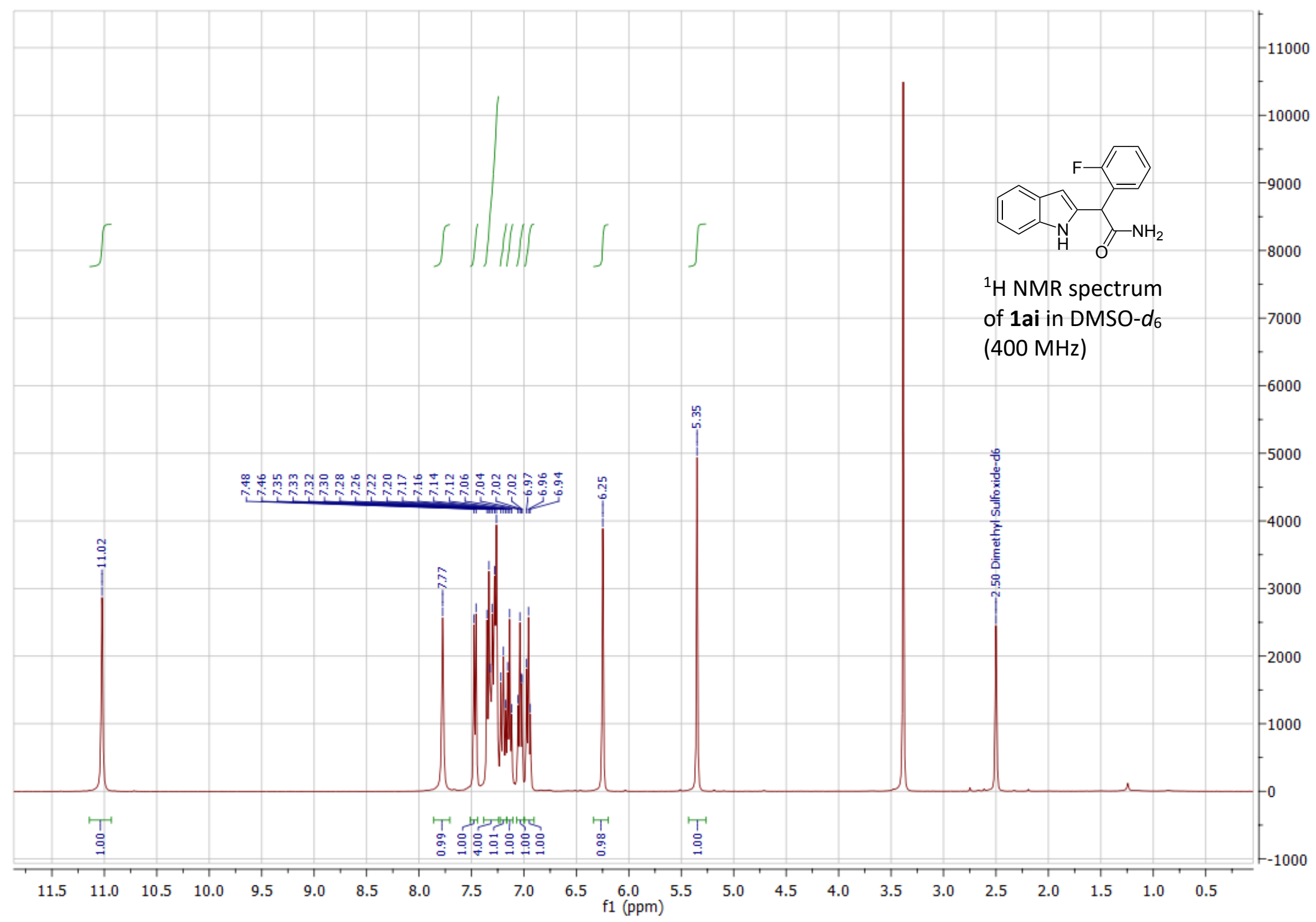




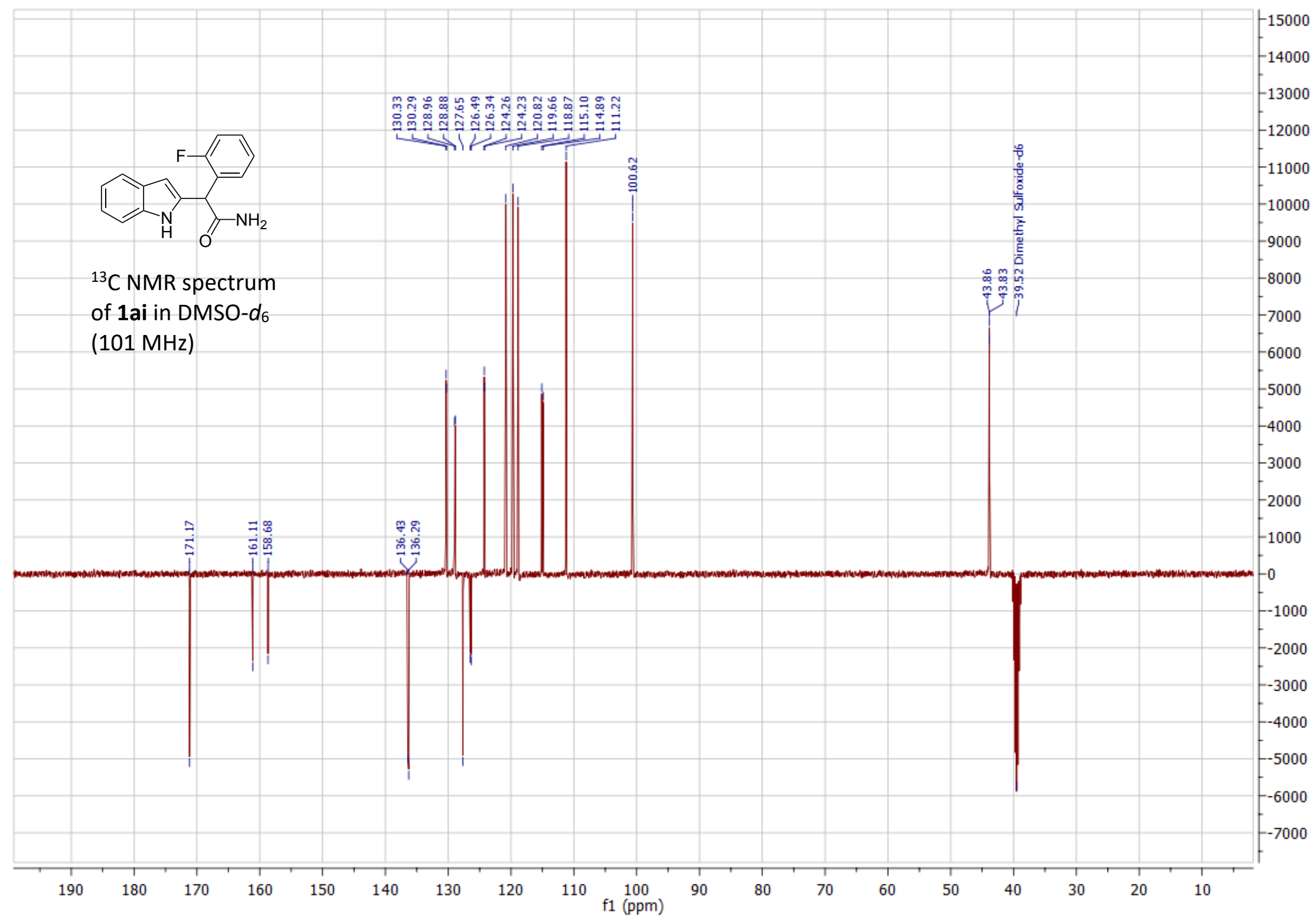




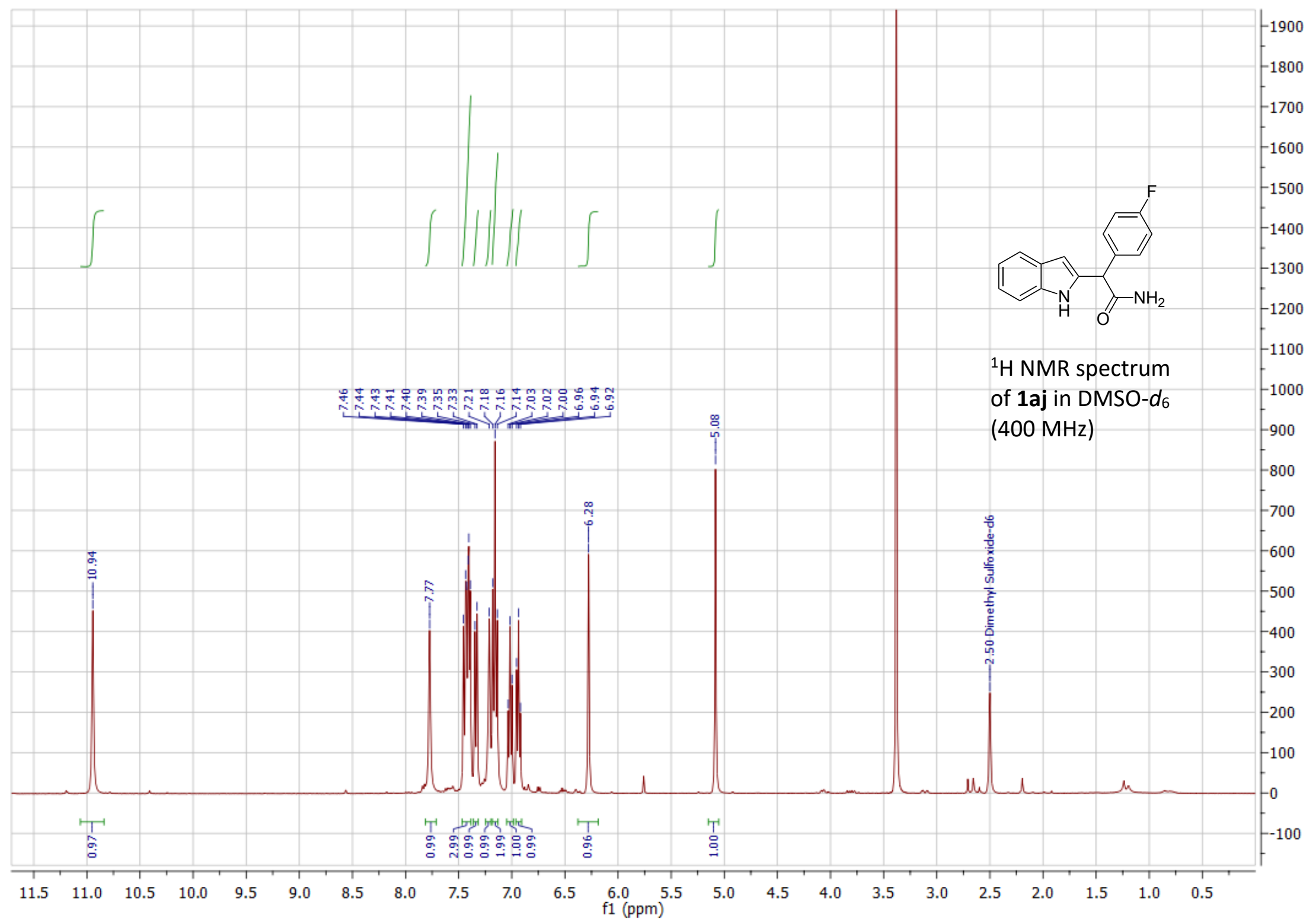




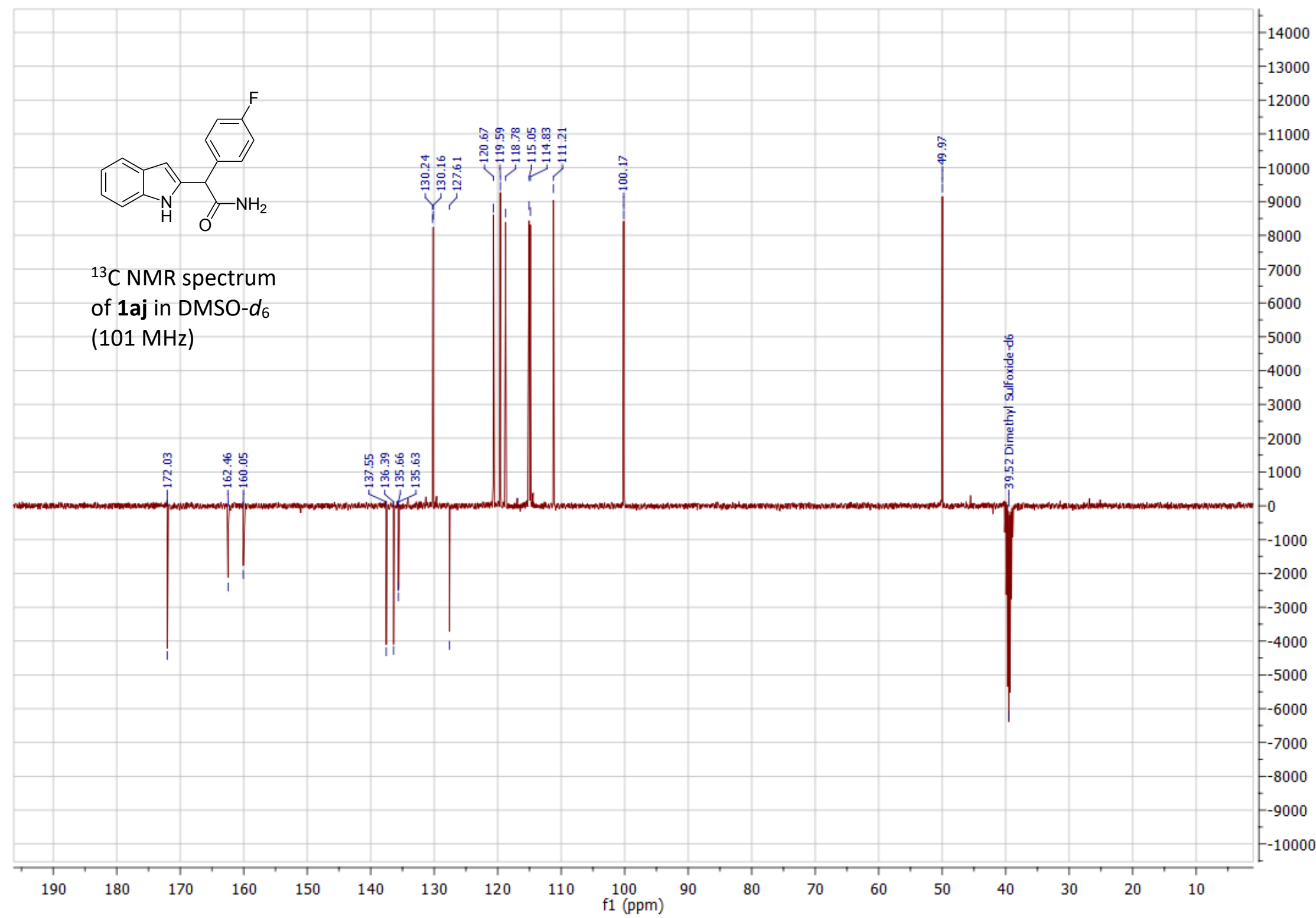




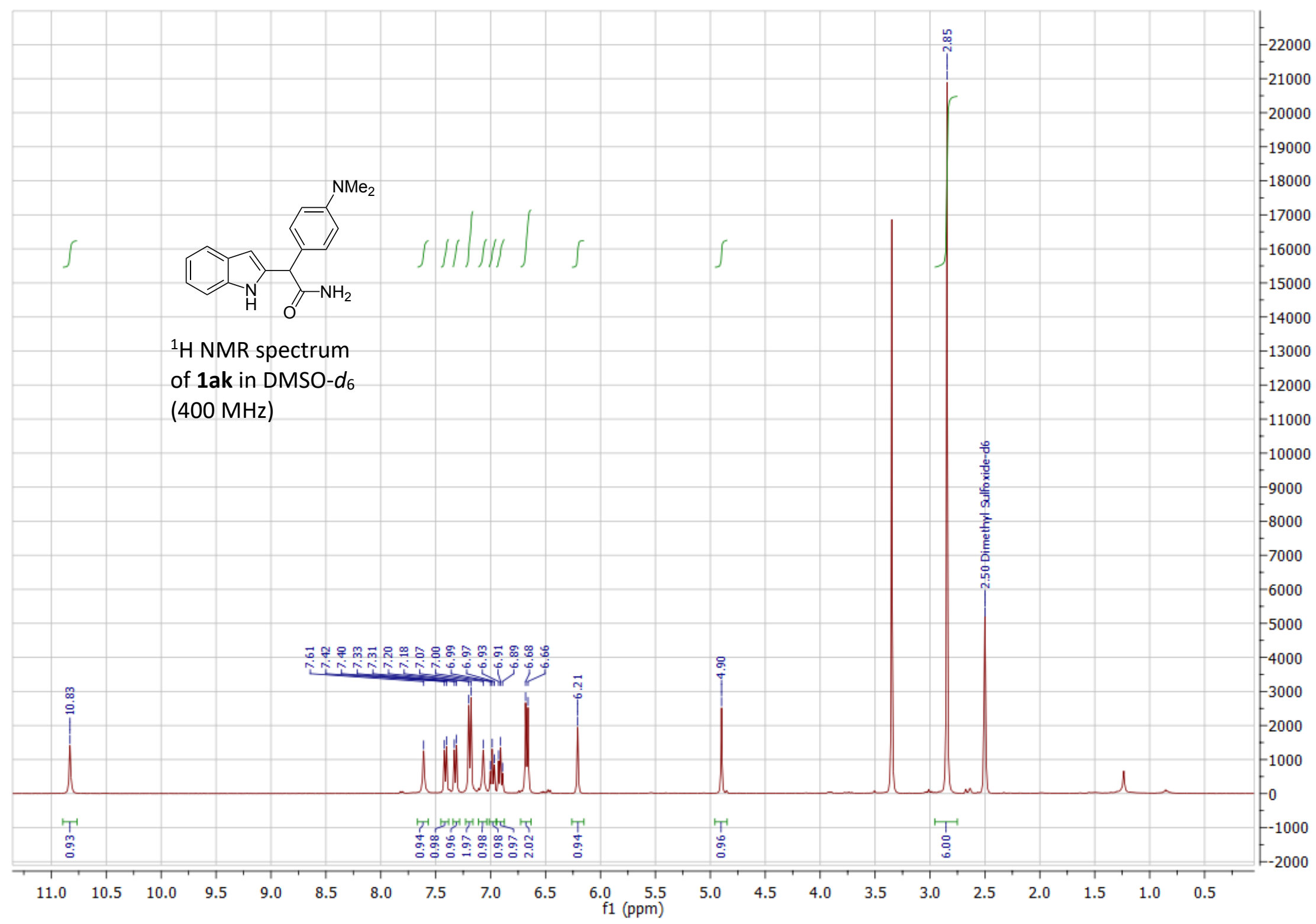




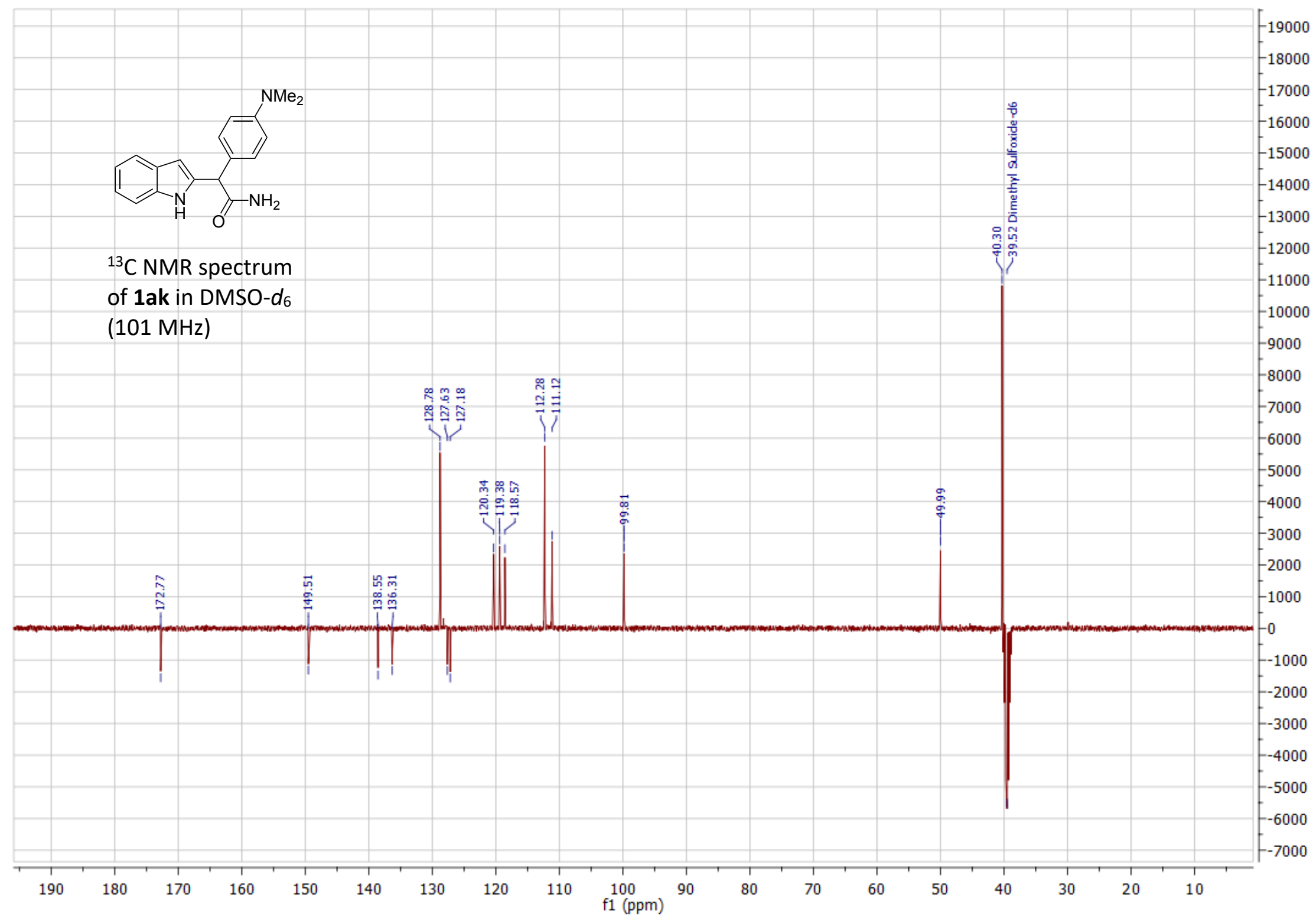




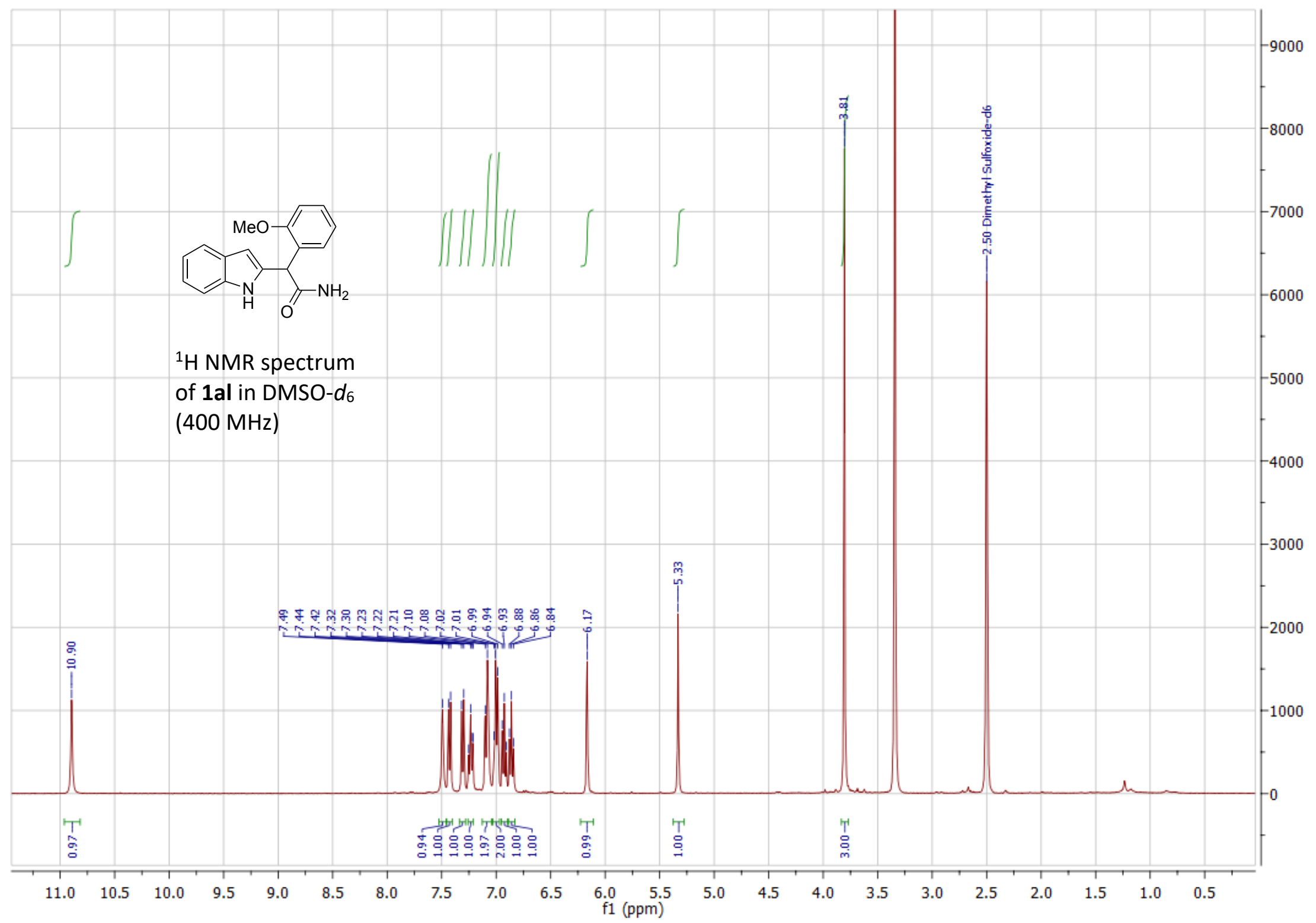




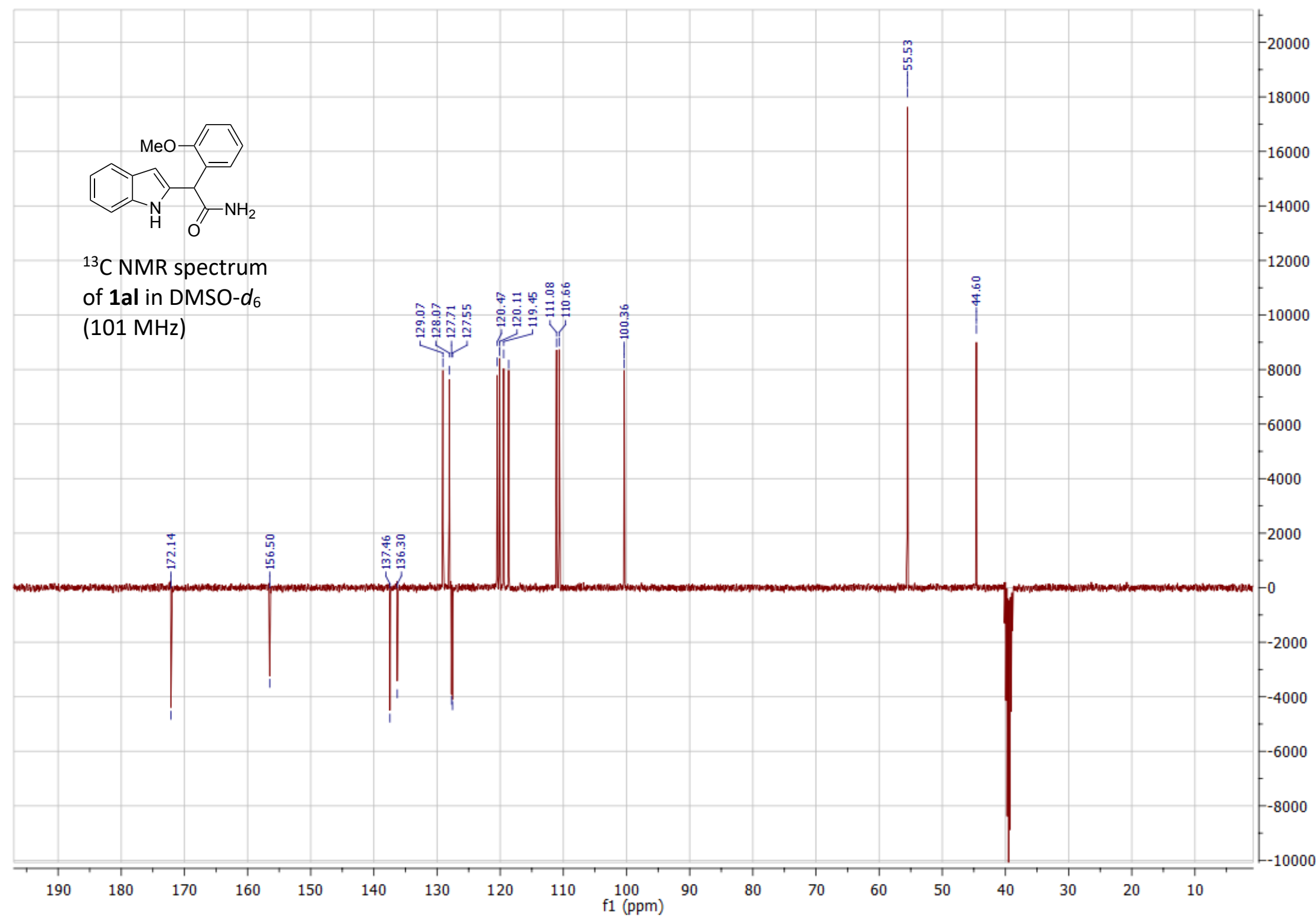




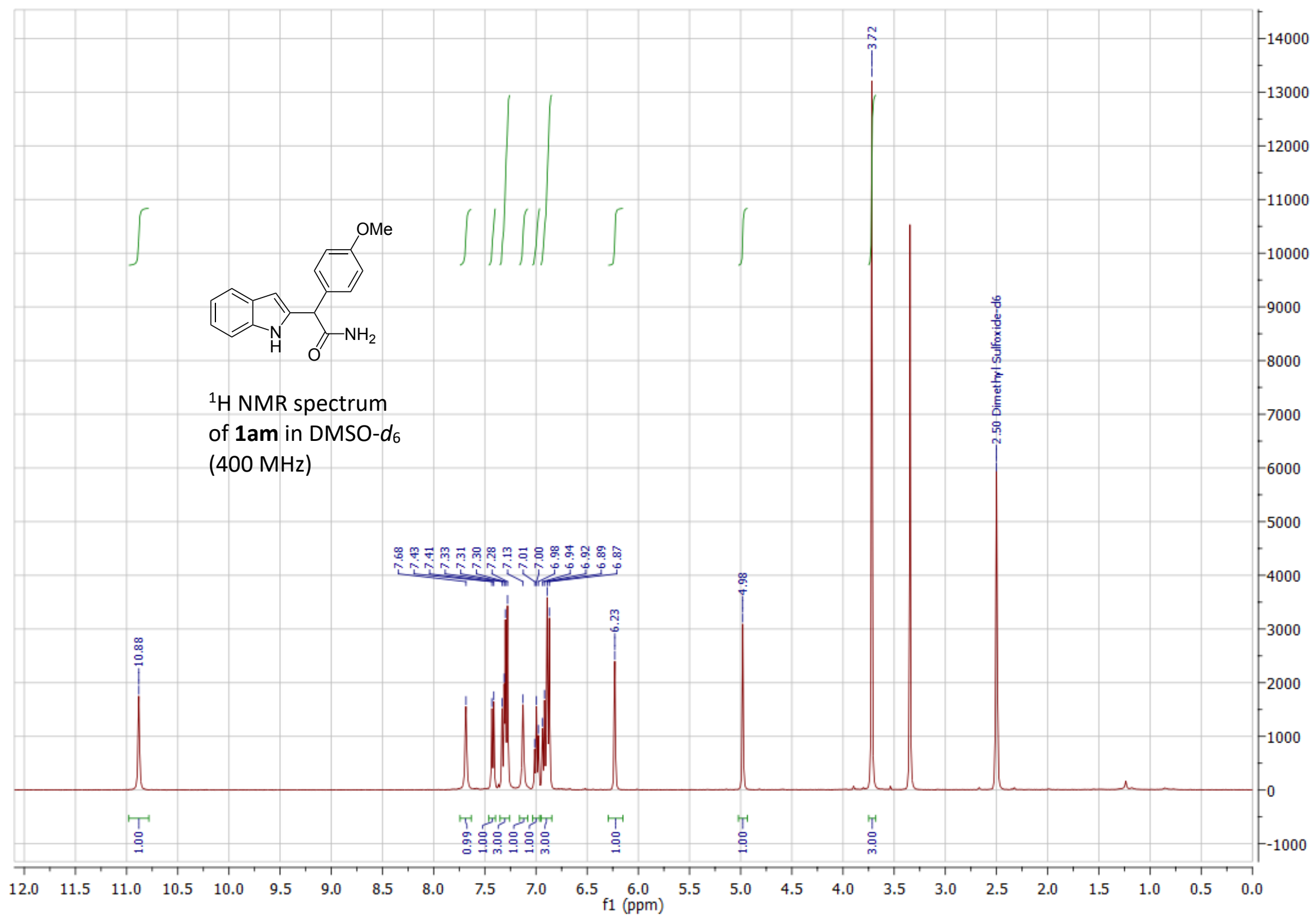




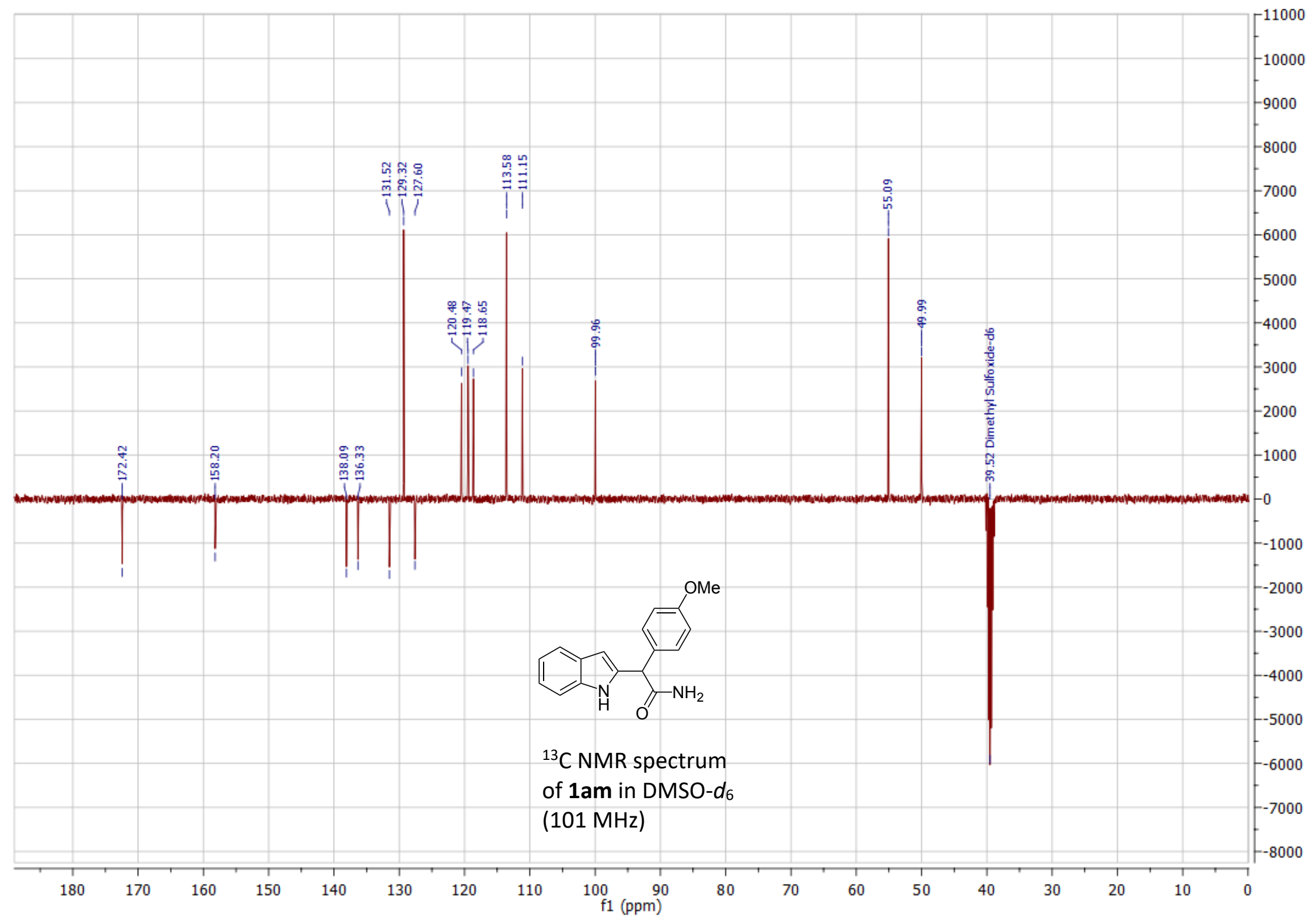




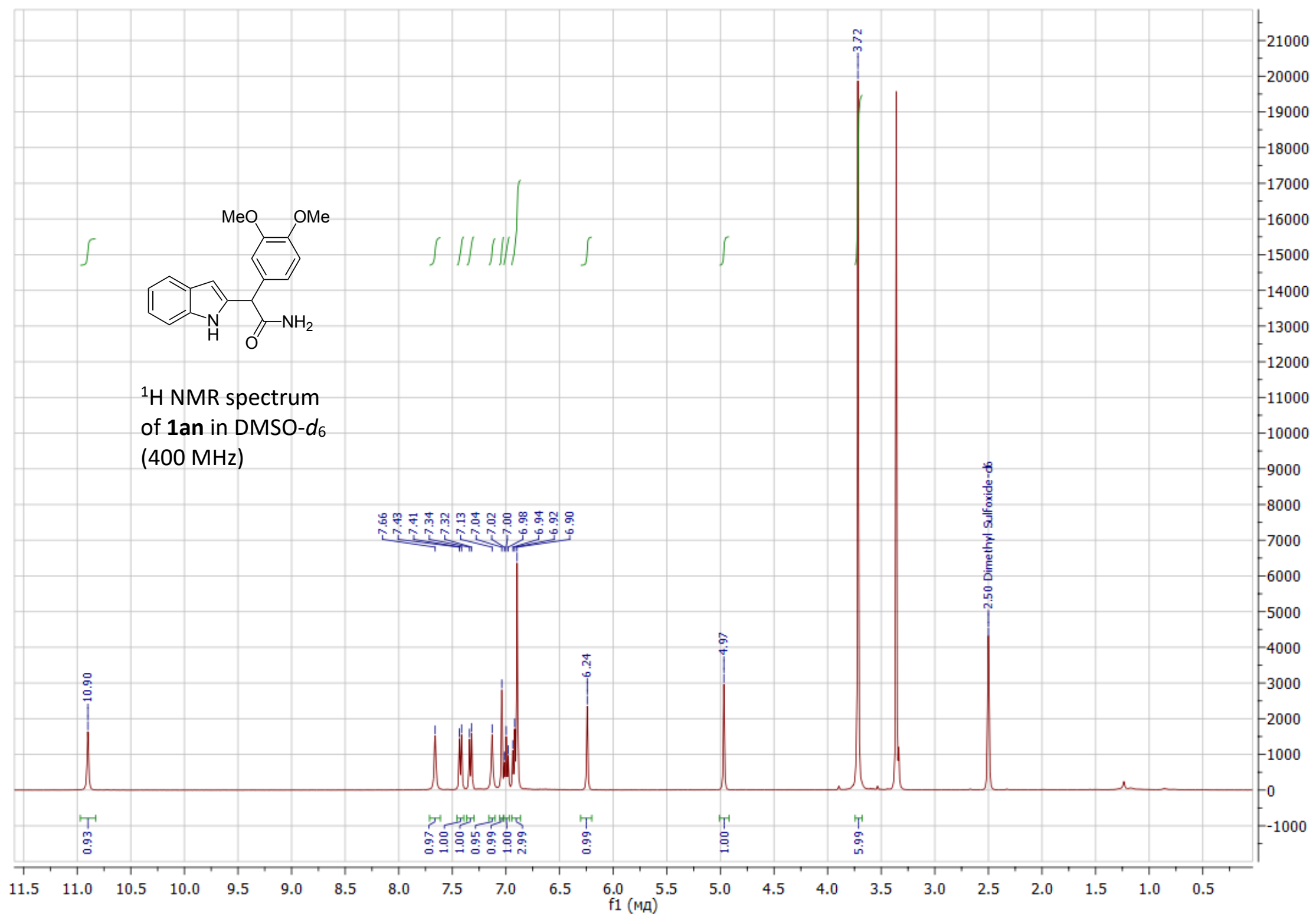




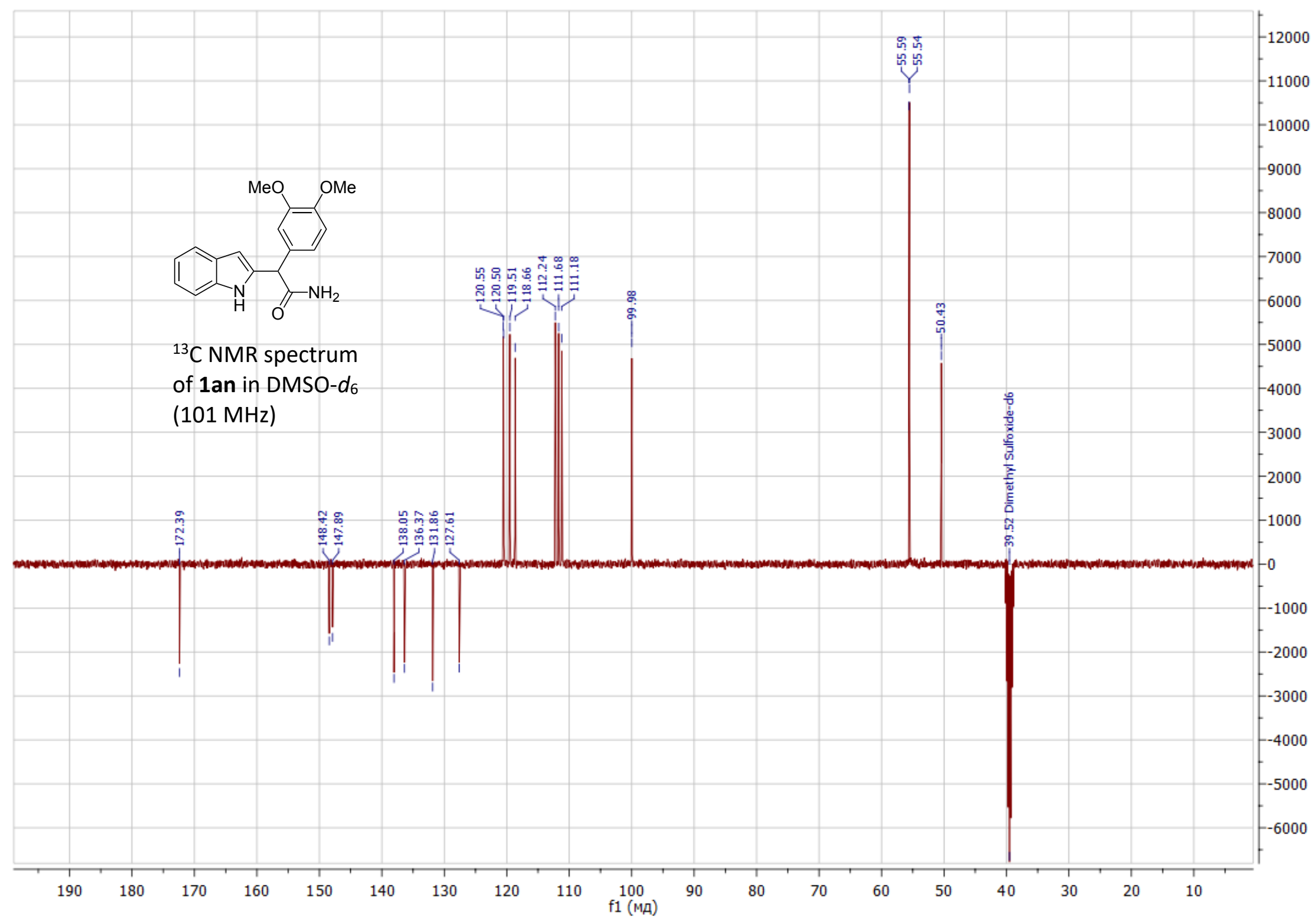




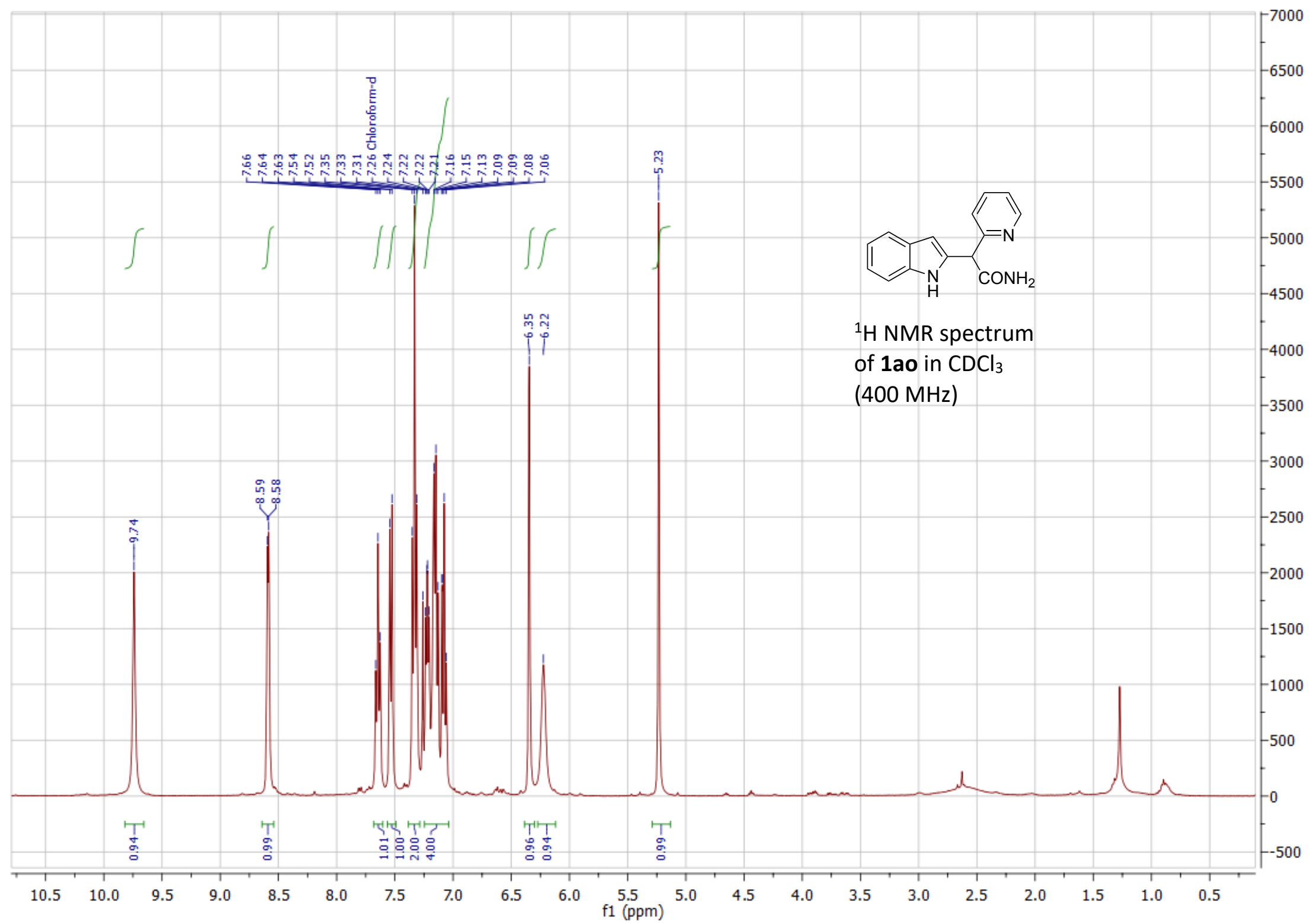




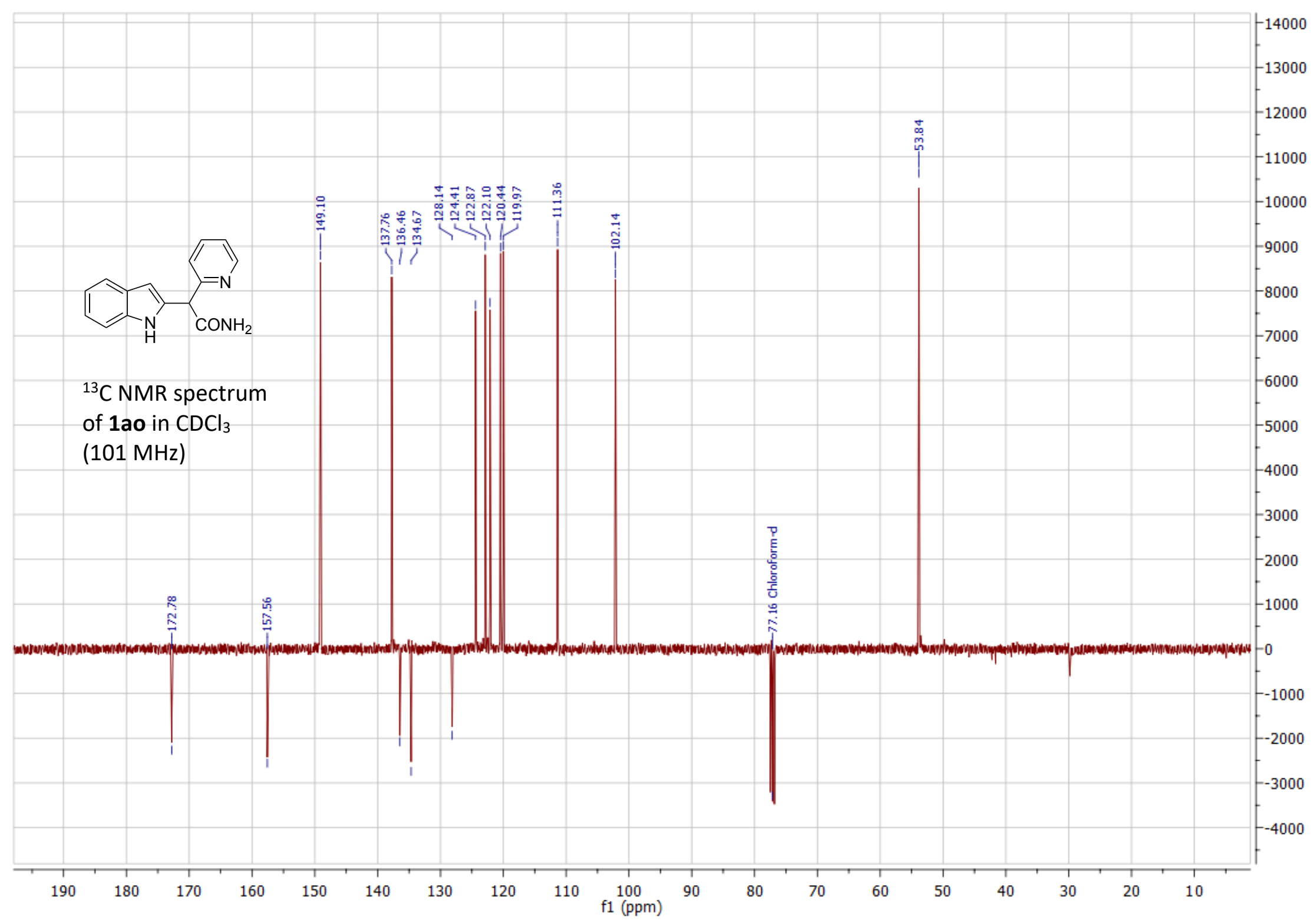




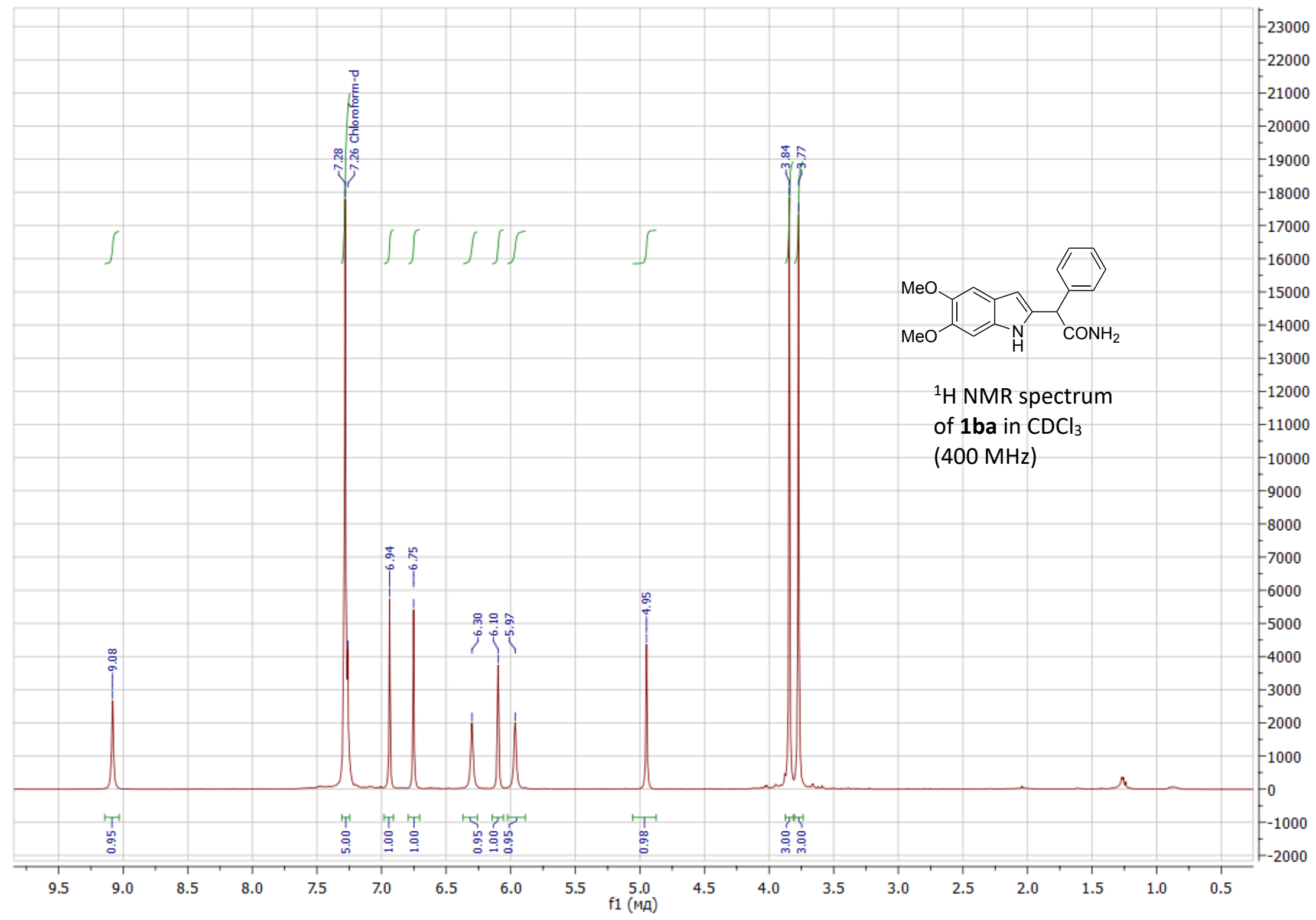




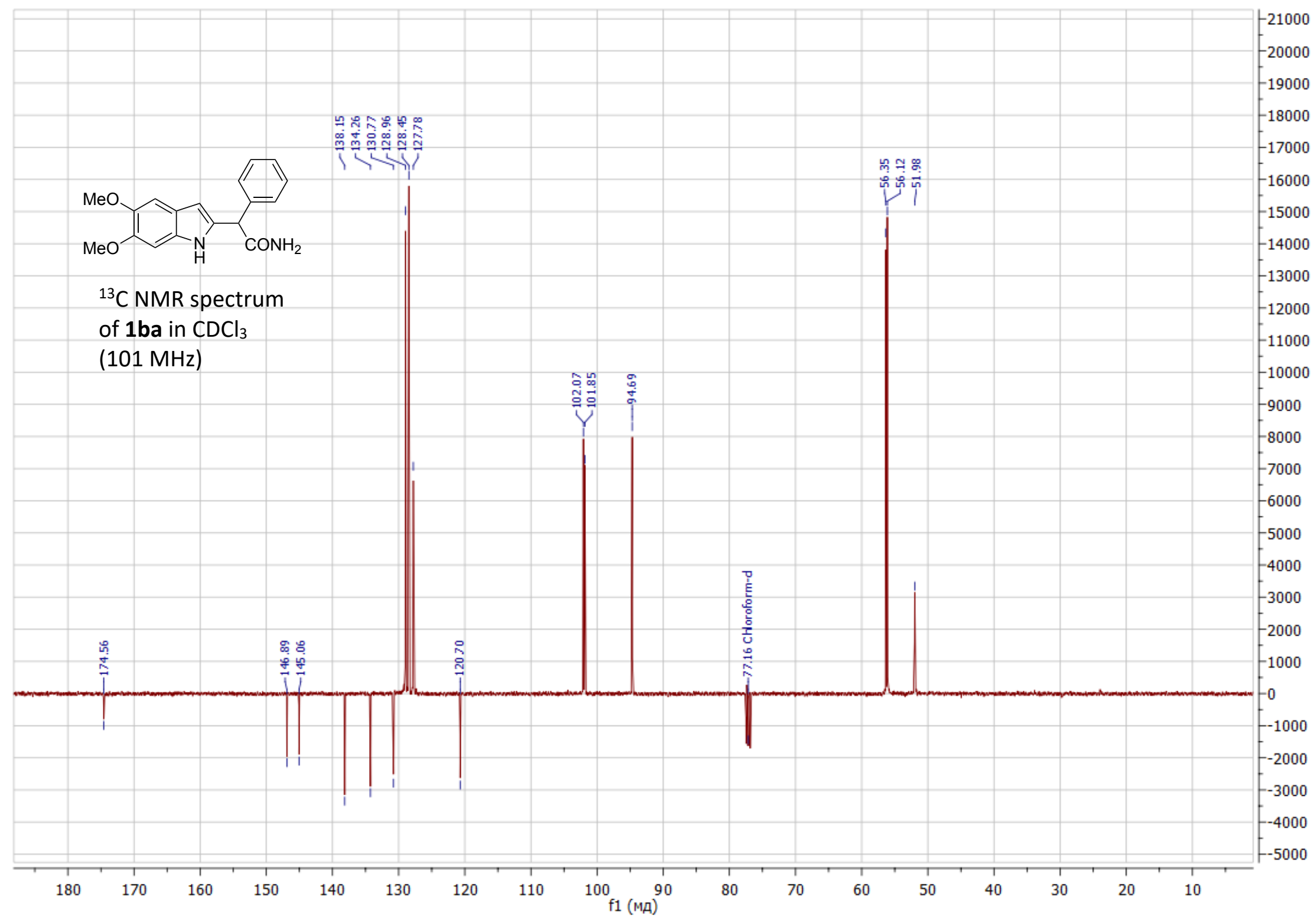




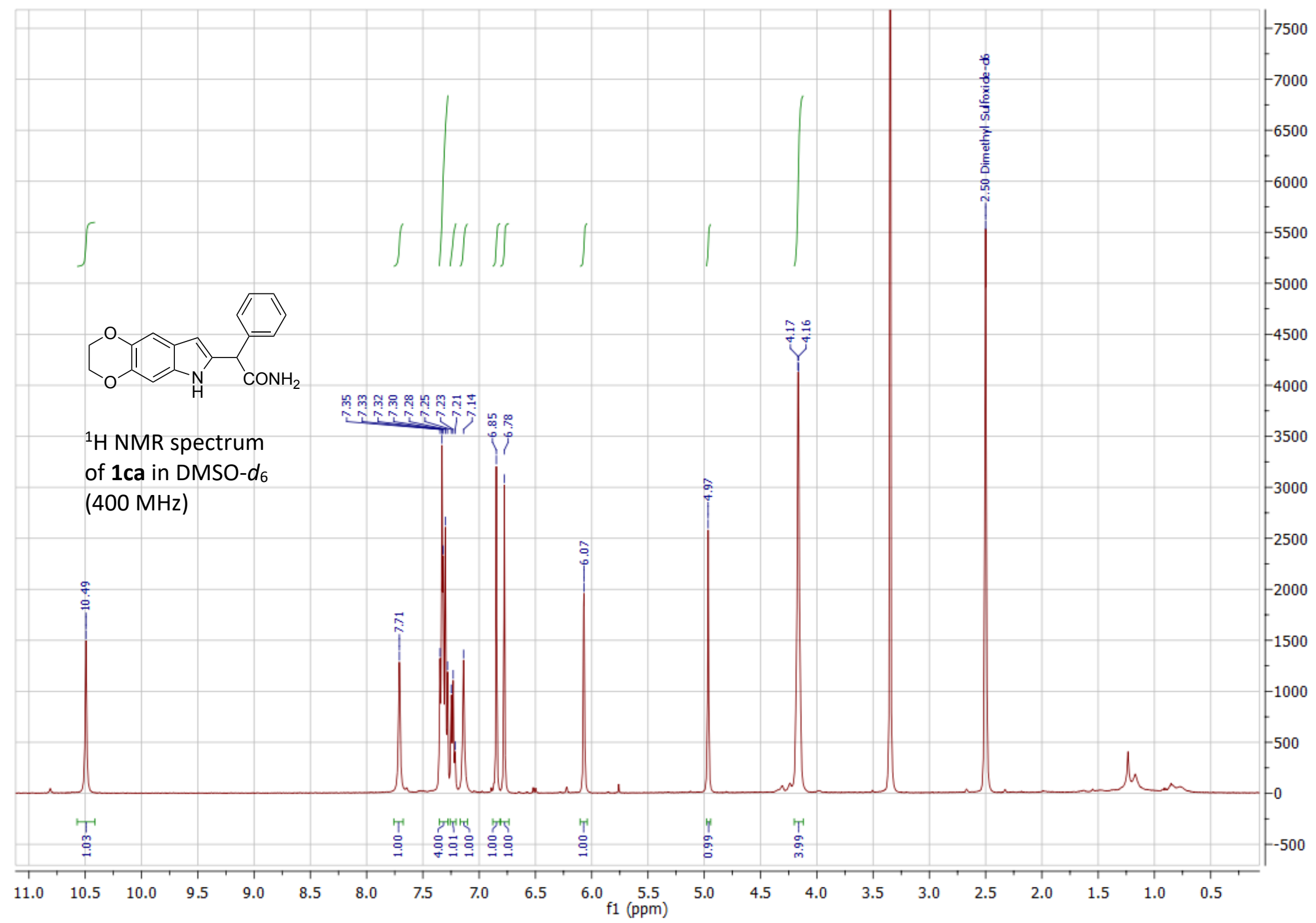




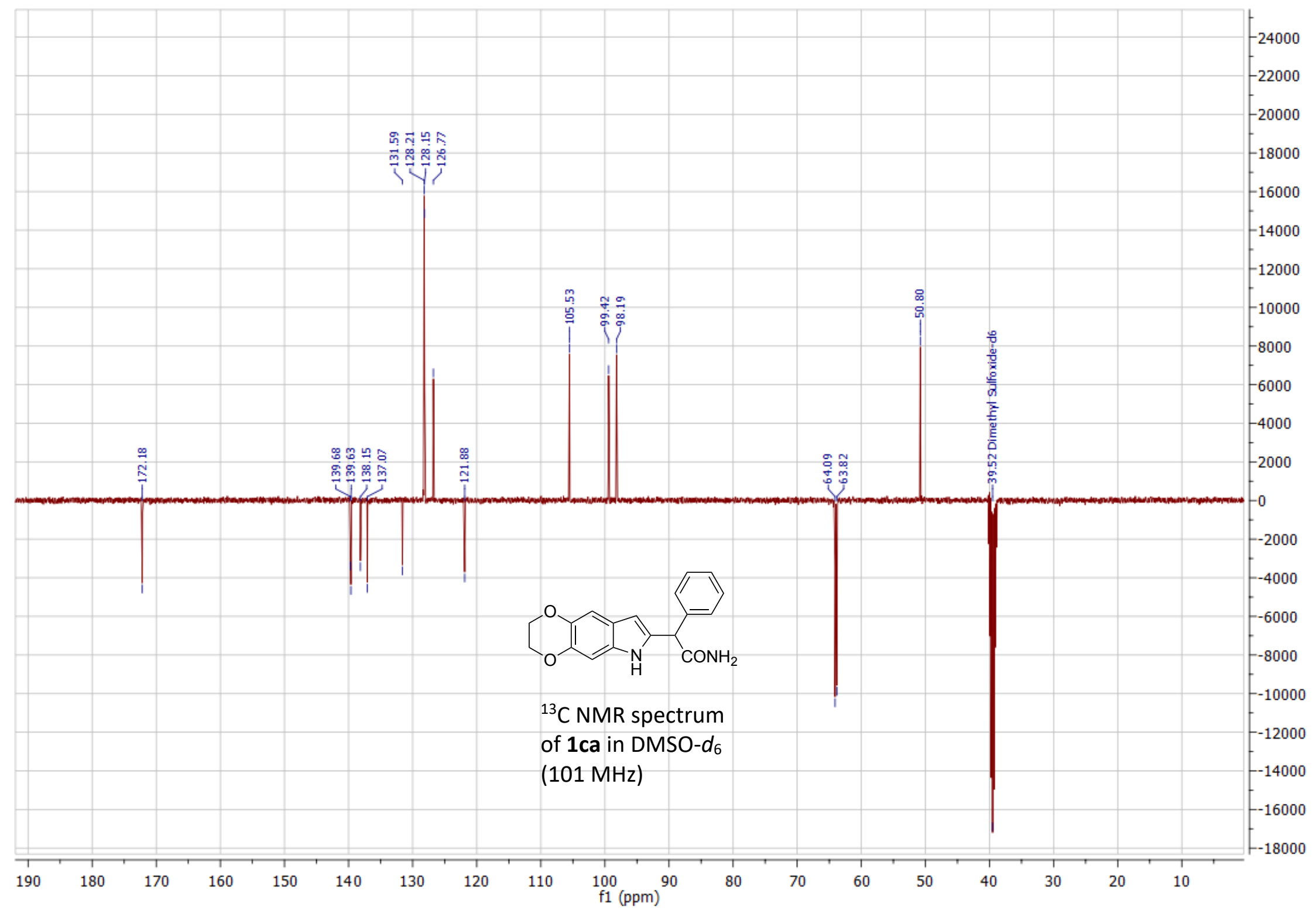




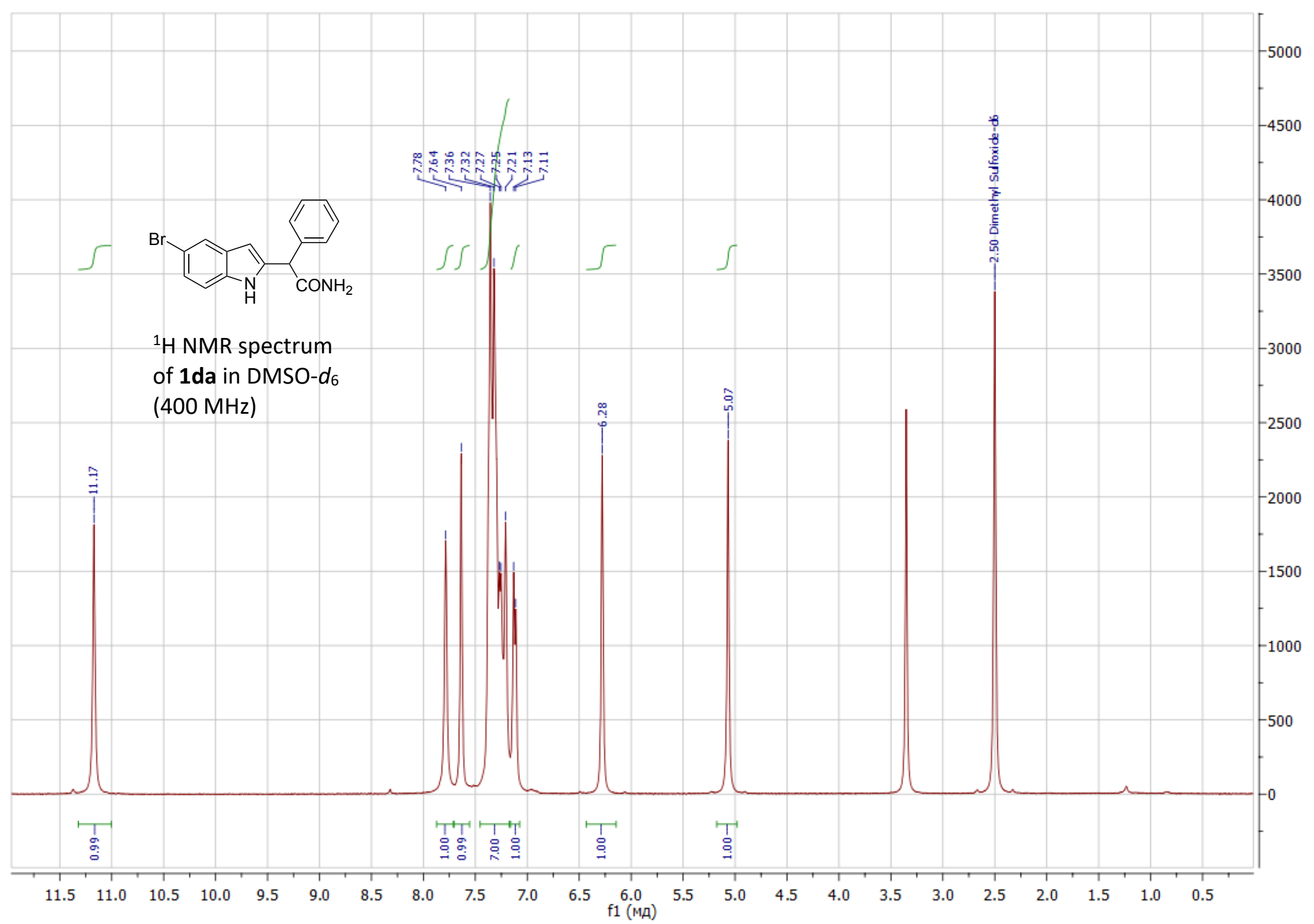




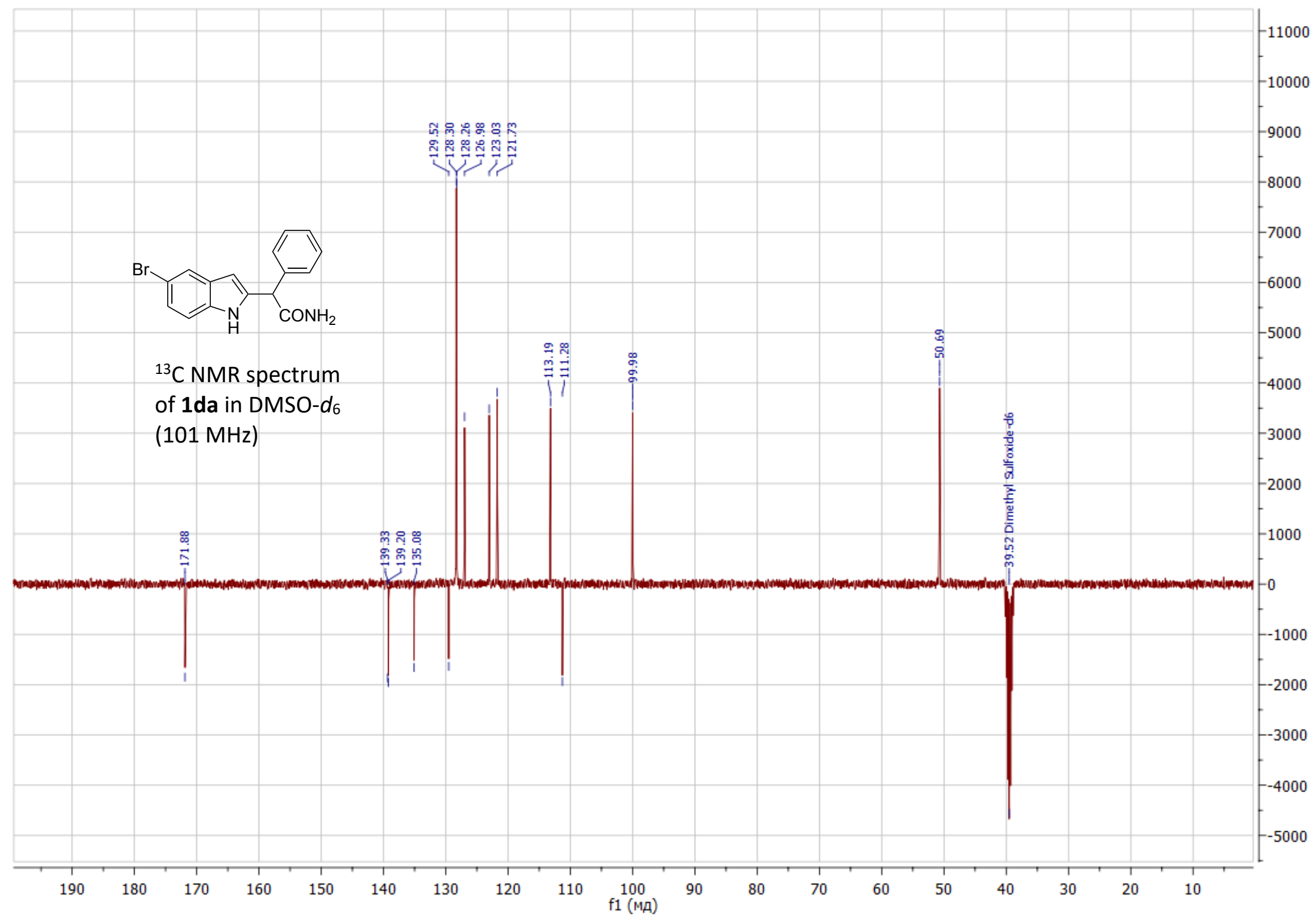


${ }^{1} \mathrm{H}$ and ${ }^{13} \mathrm{C}$ Spectral Charts of 4-(2-aminophenyl)-4-oxo-2-arylbutanenitriles 4

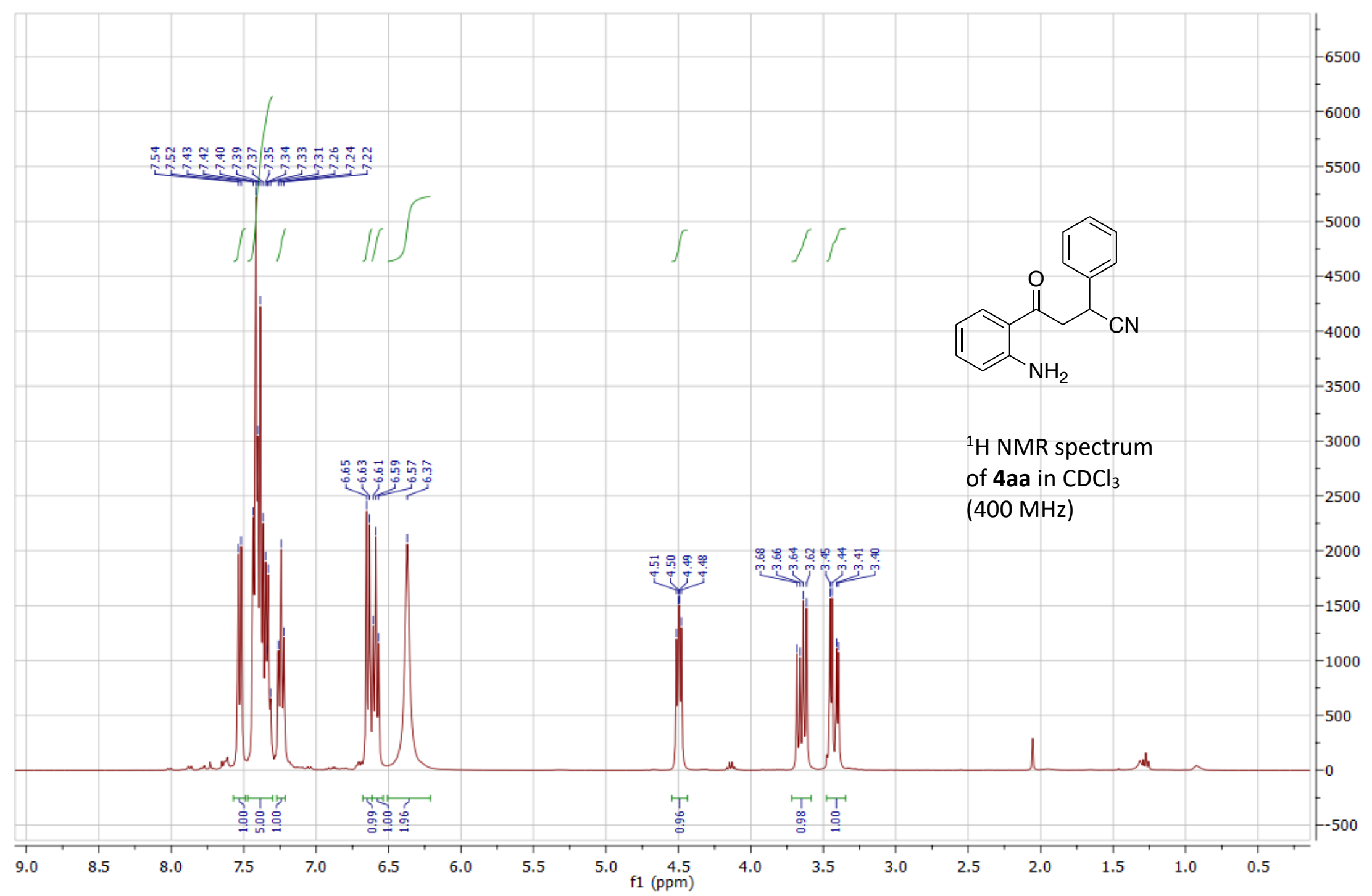




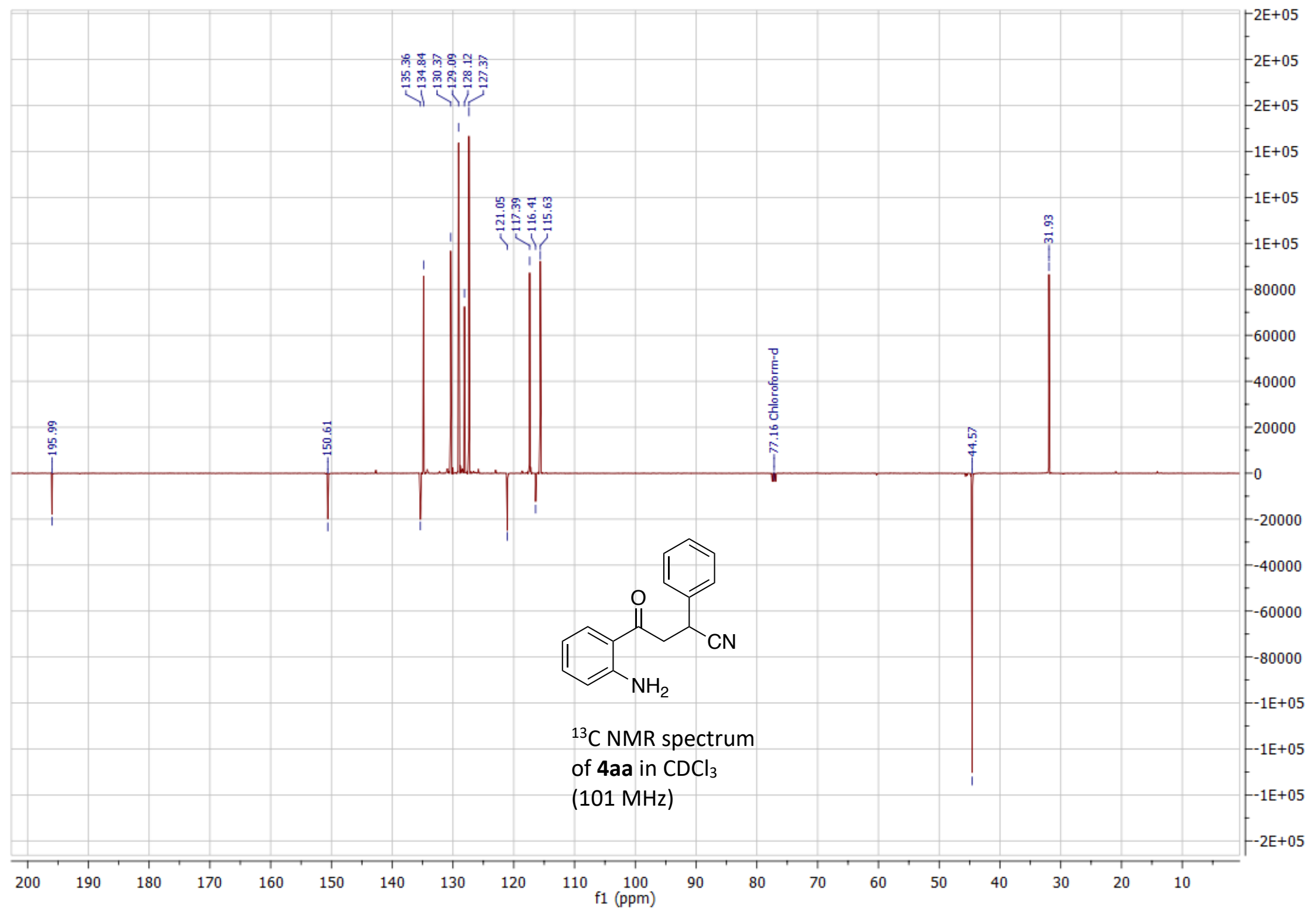




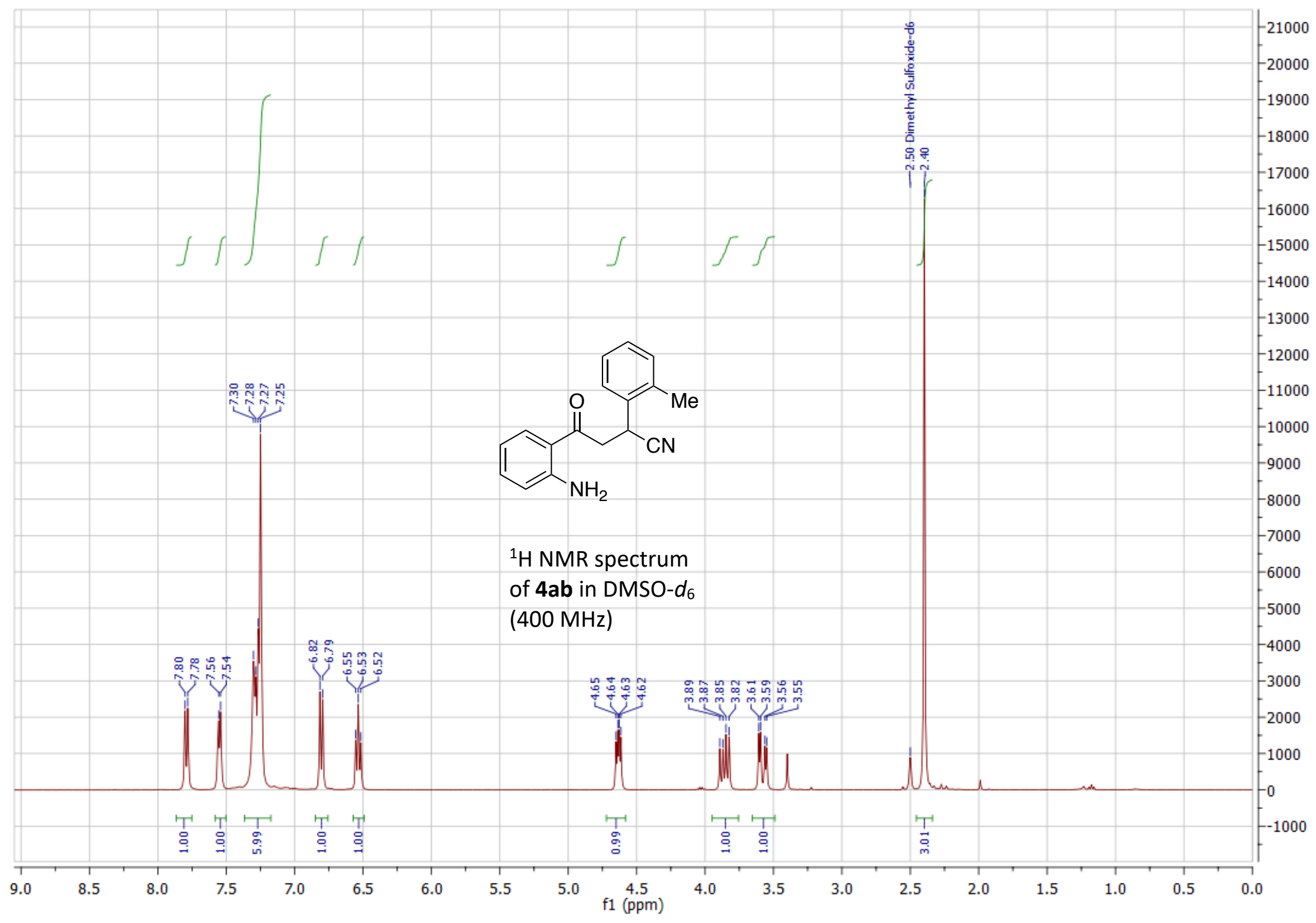




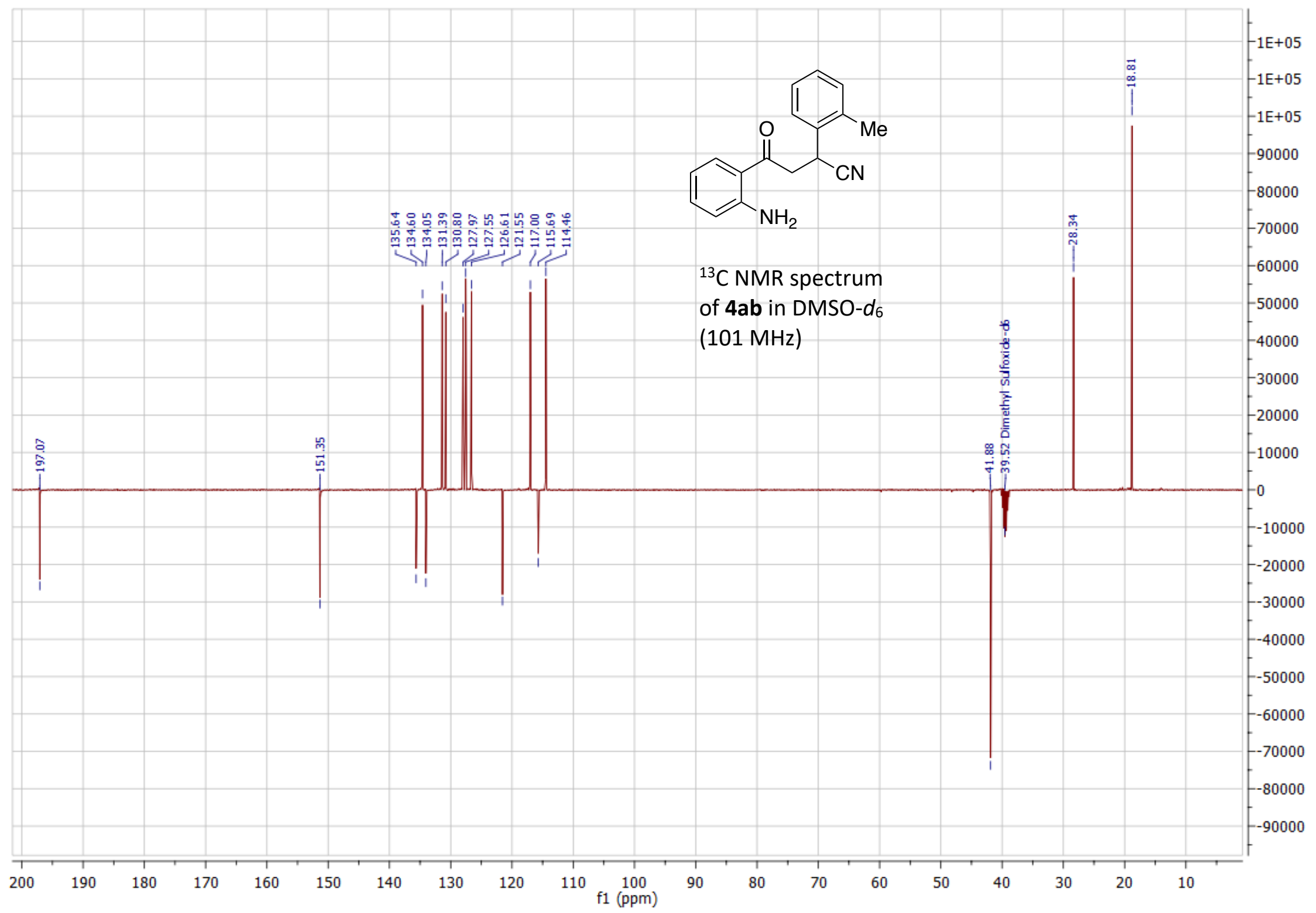




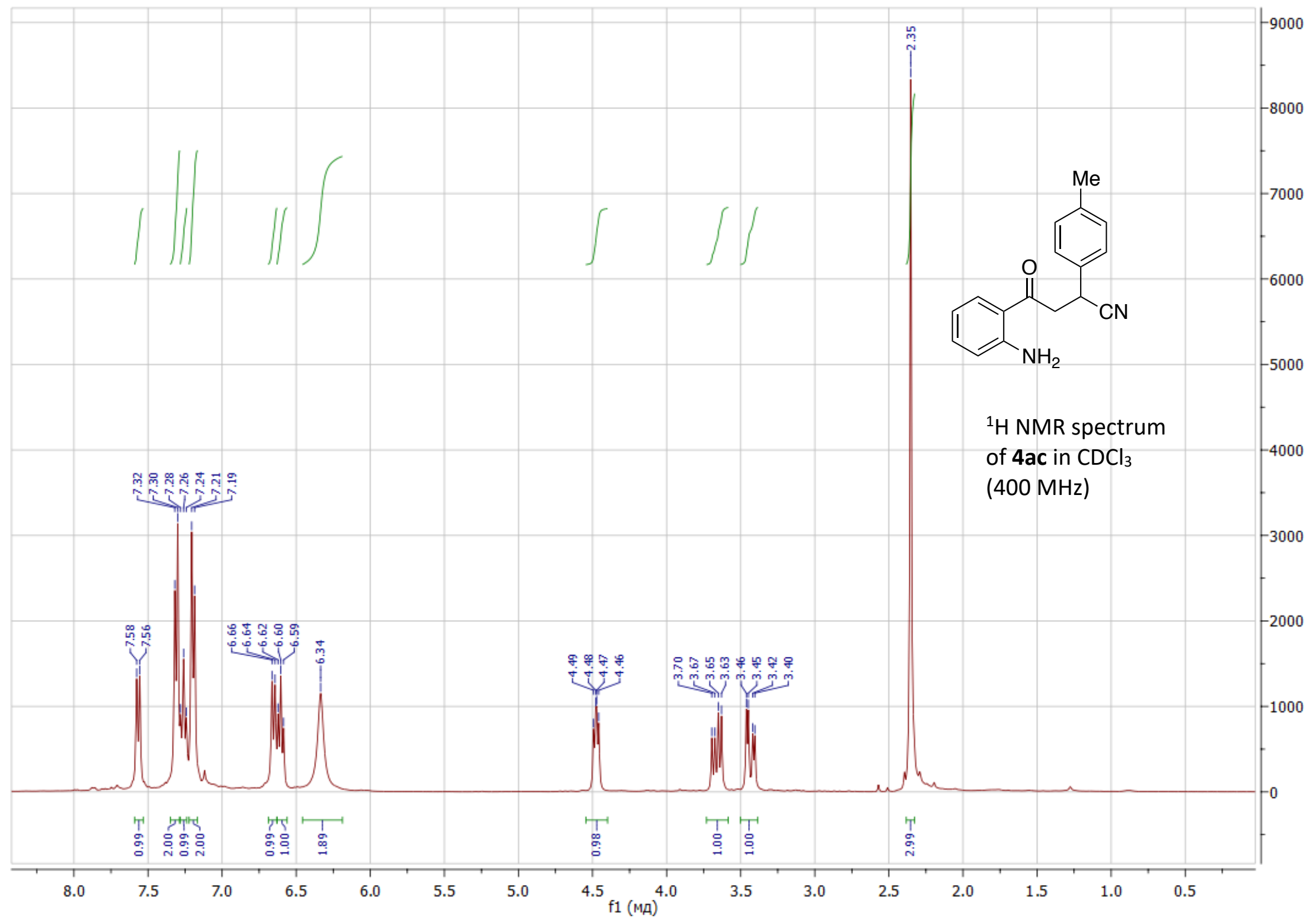




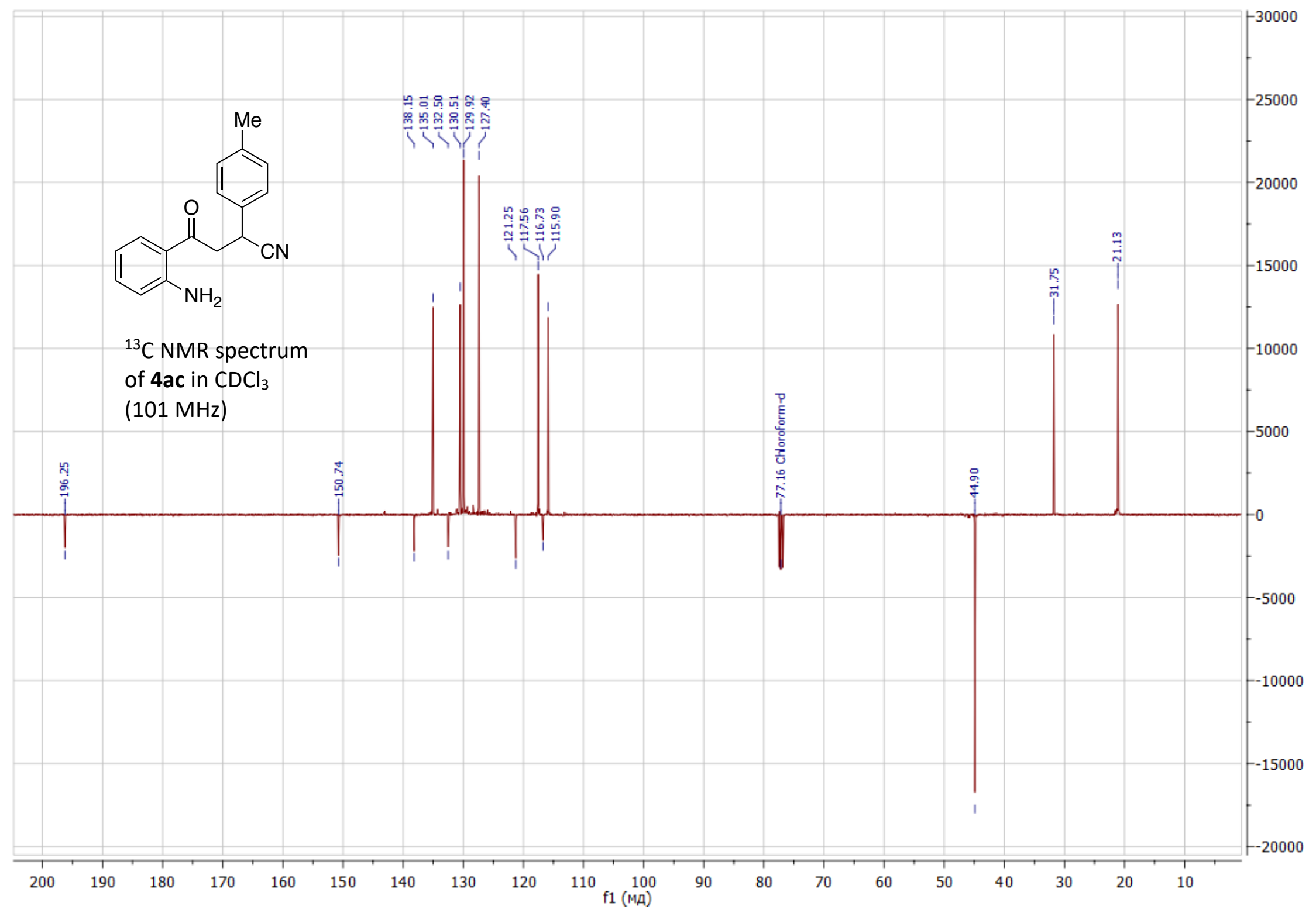




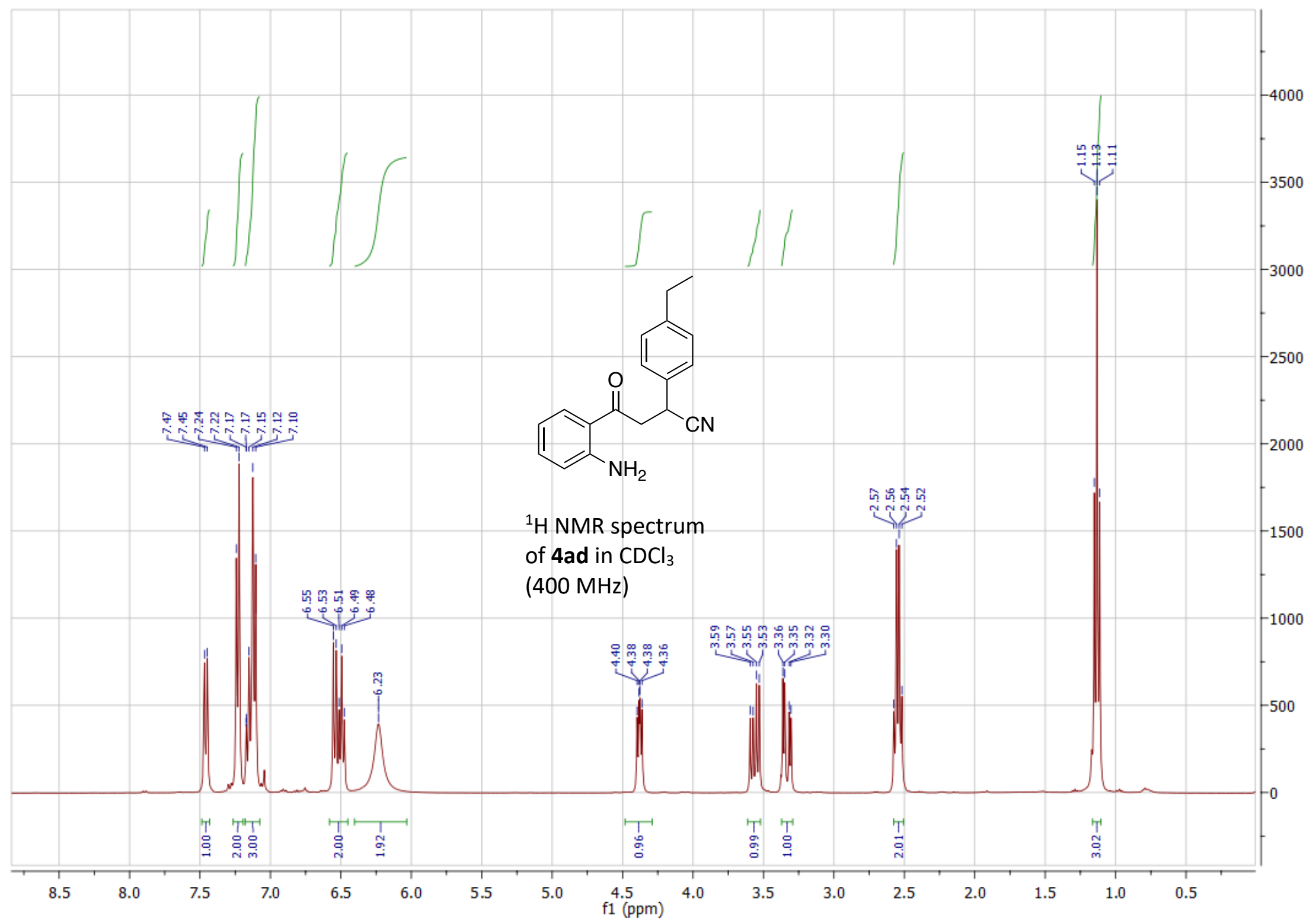




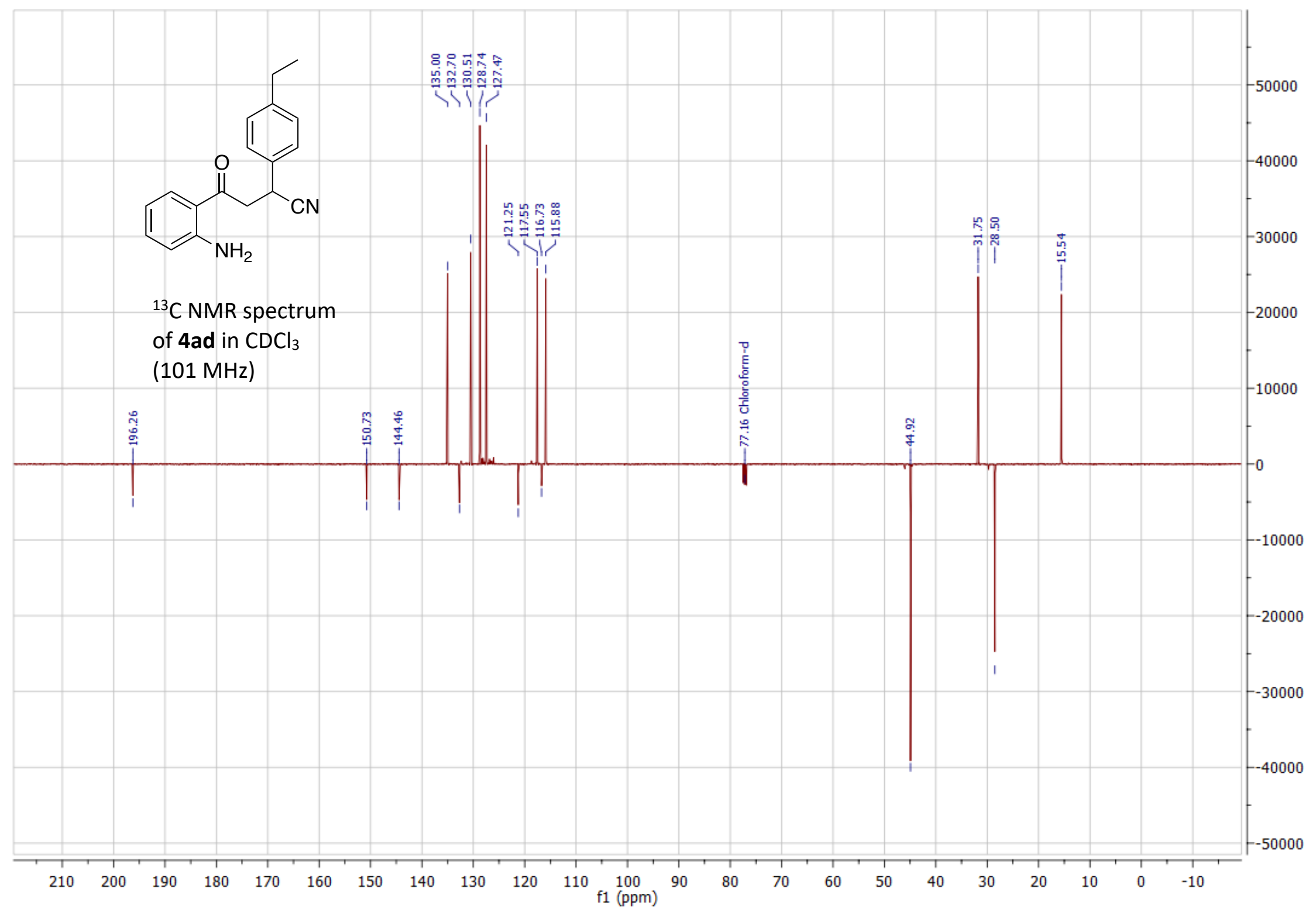




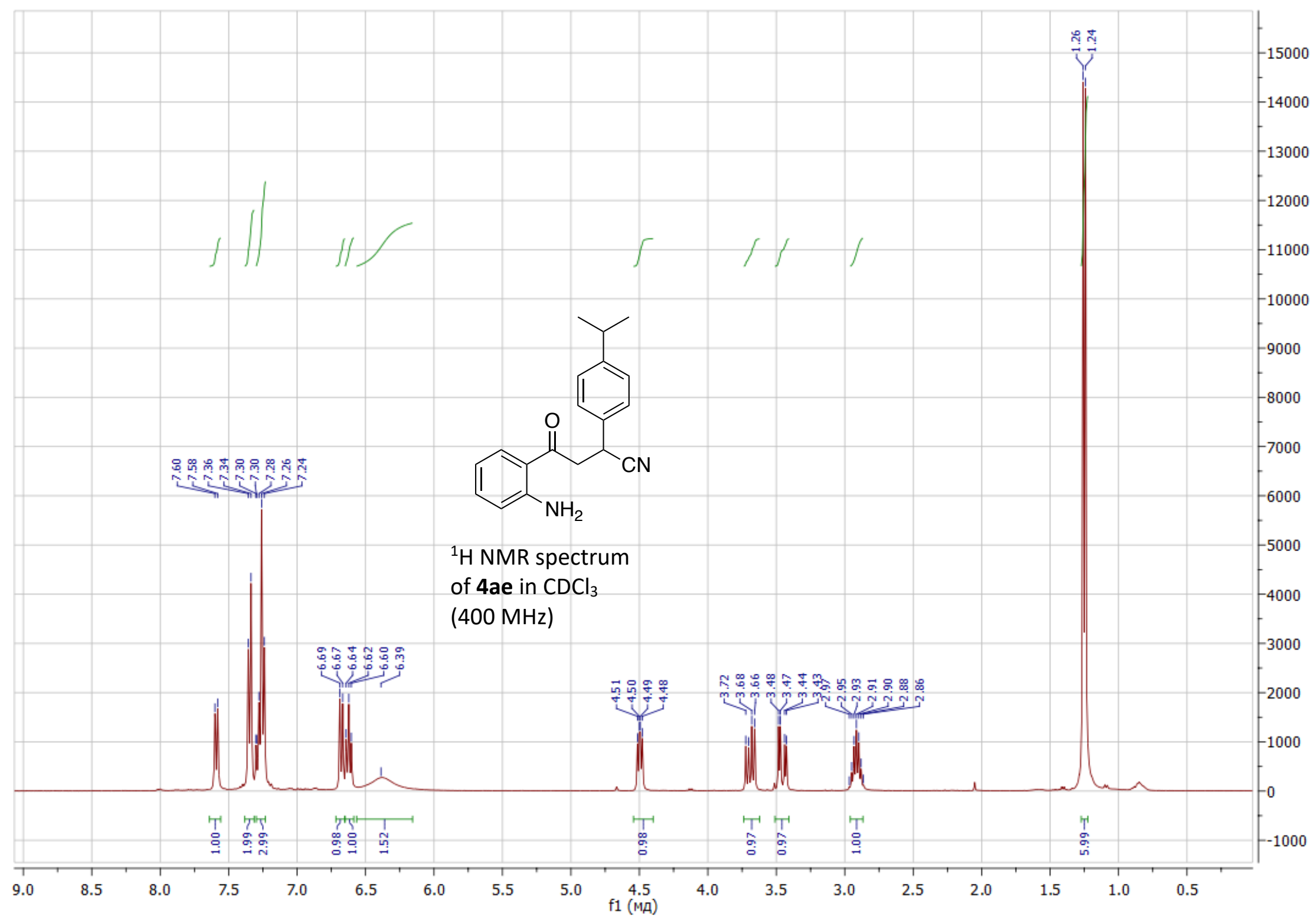




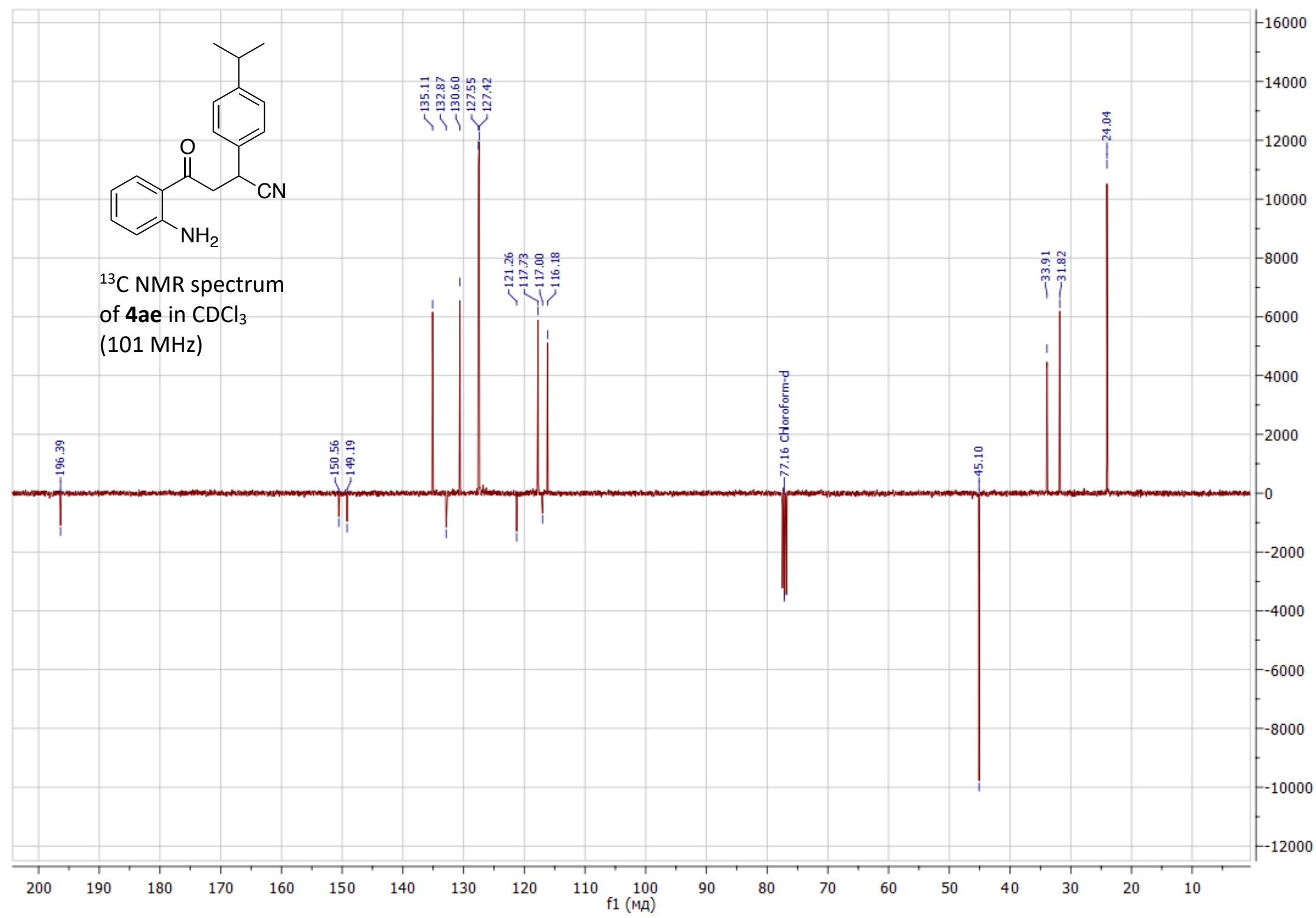




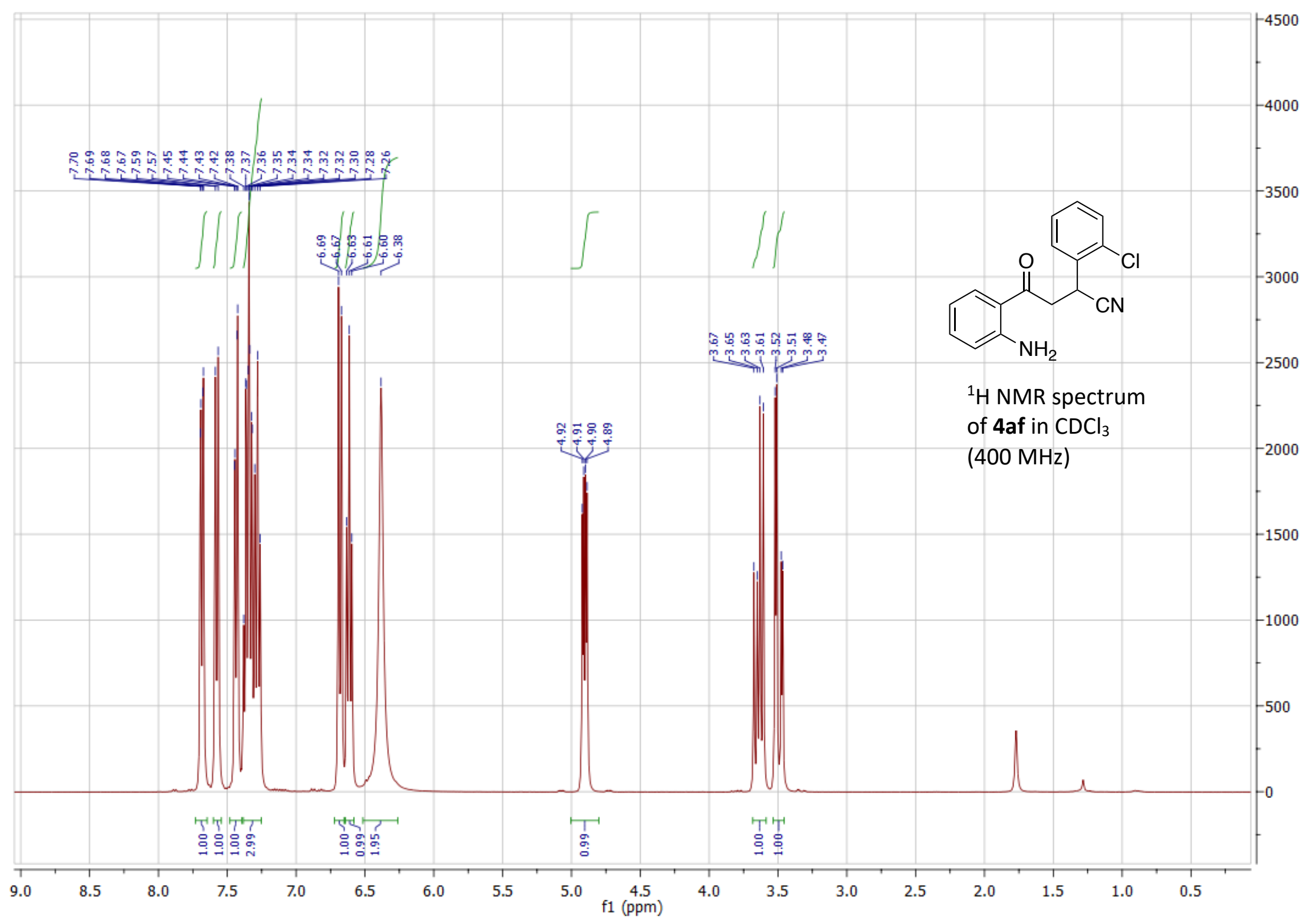




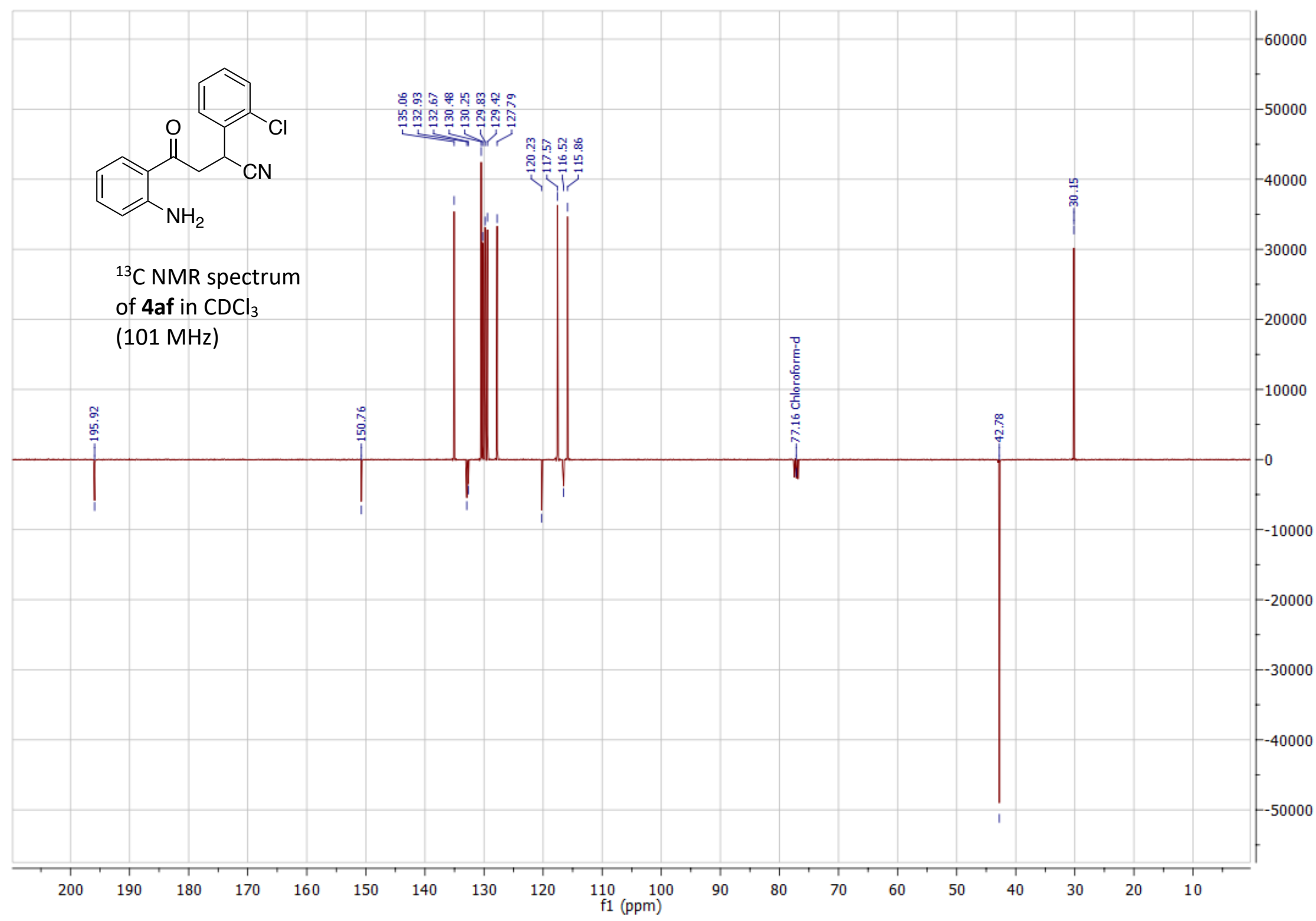




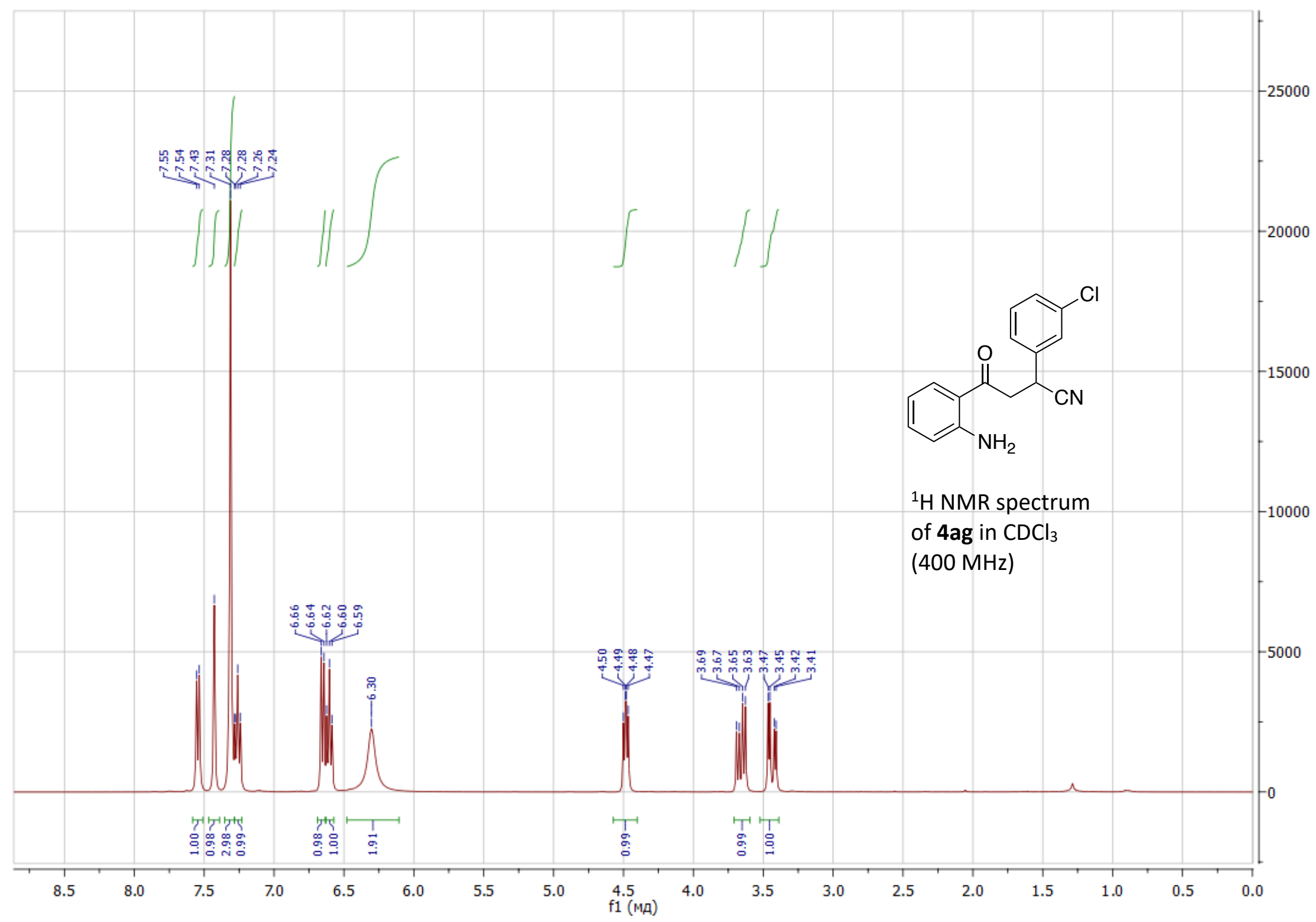




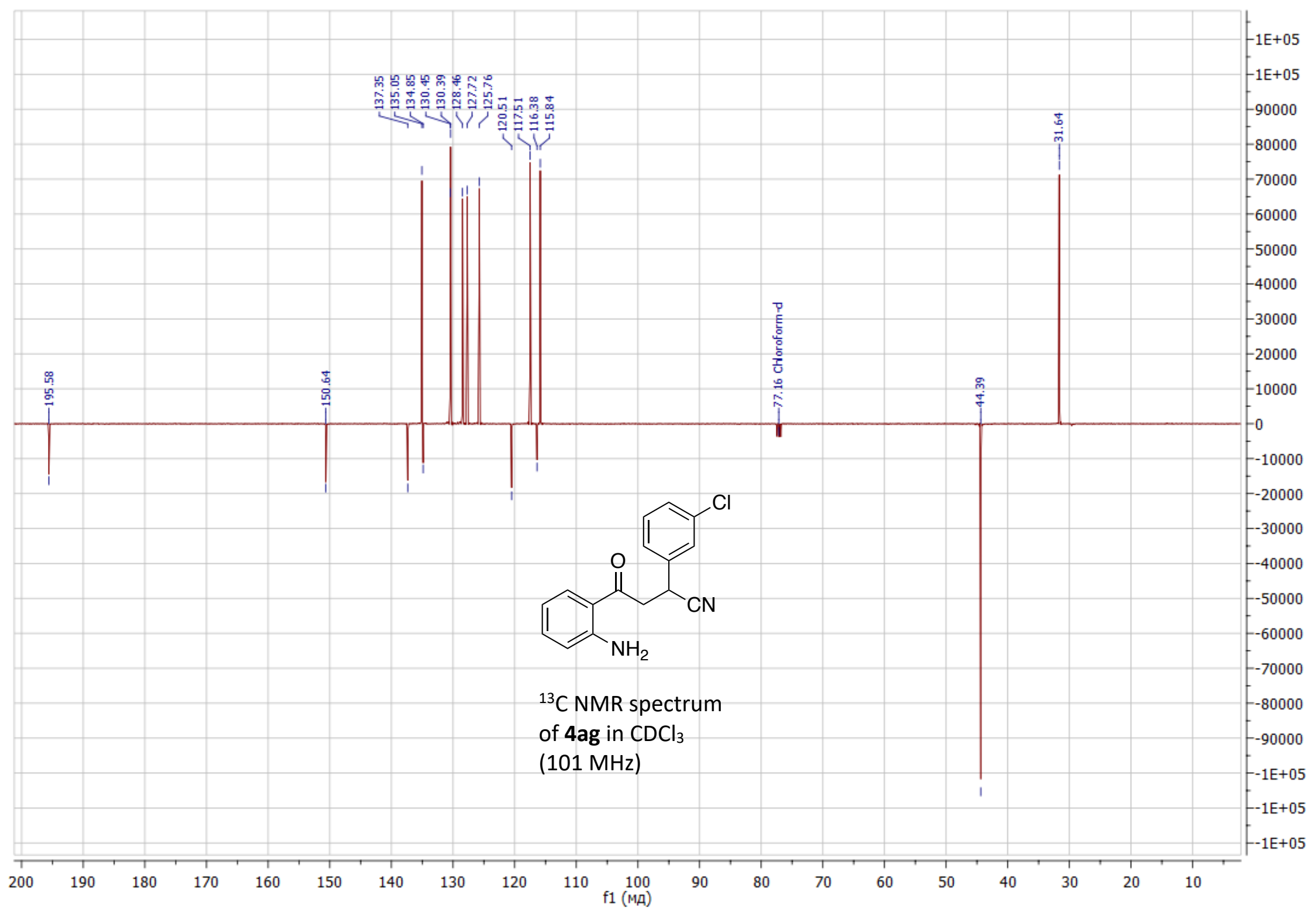




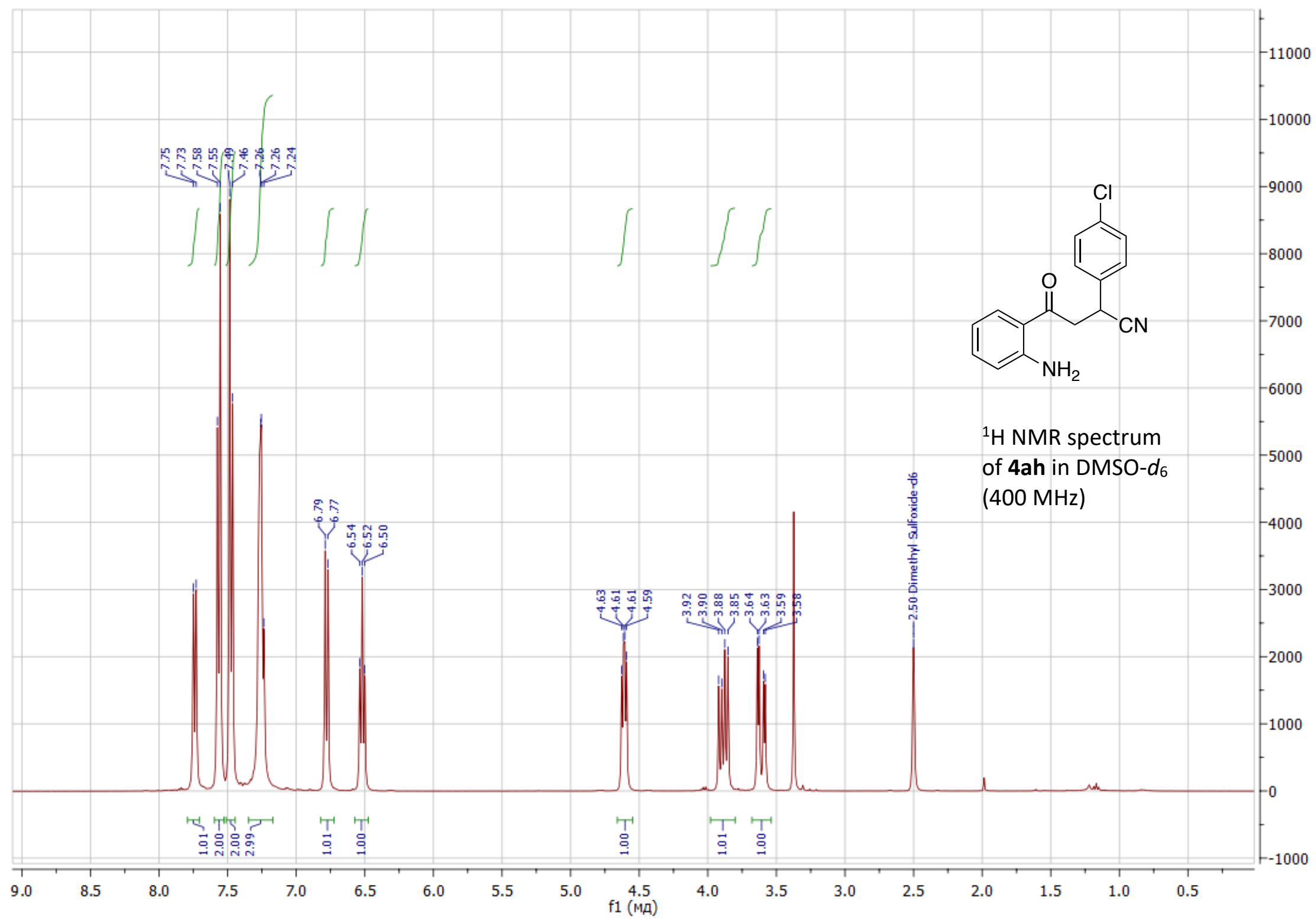




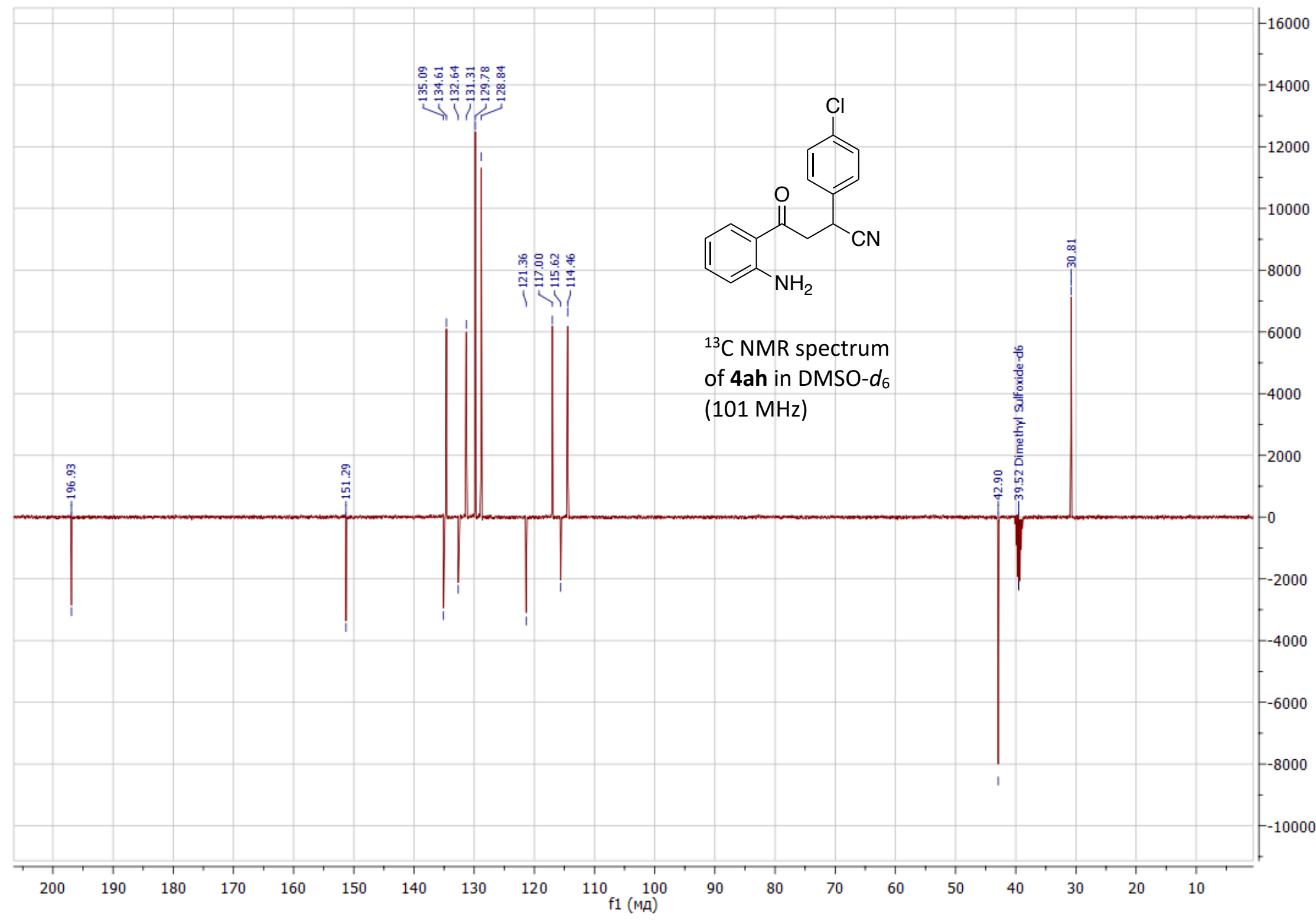




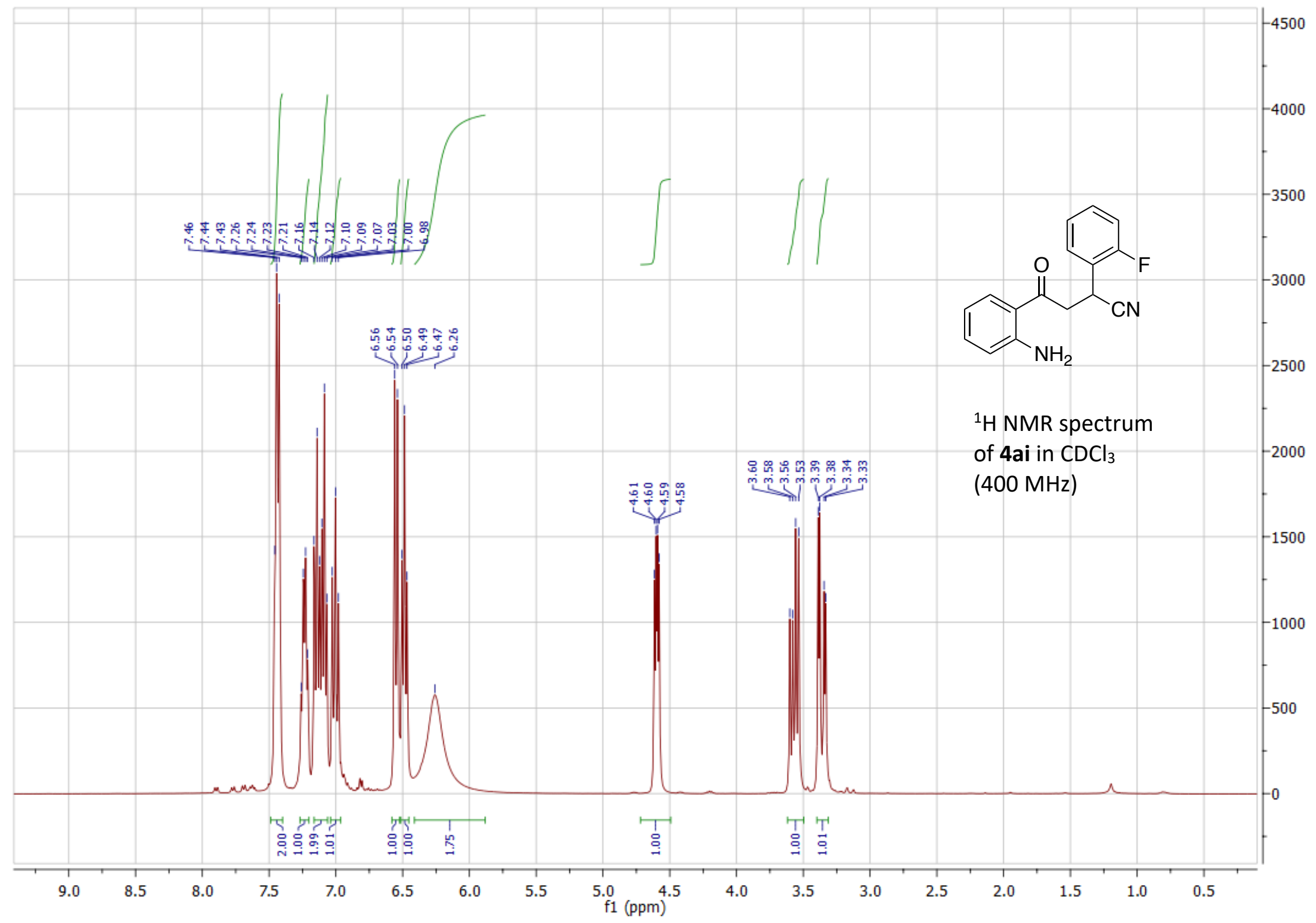




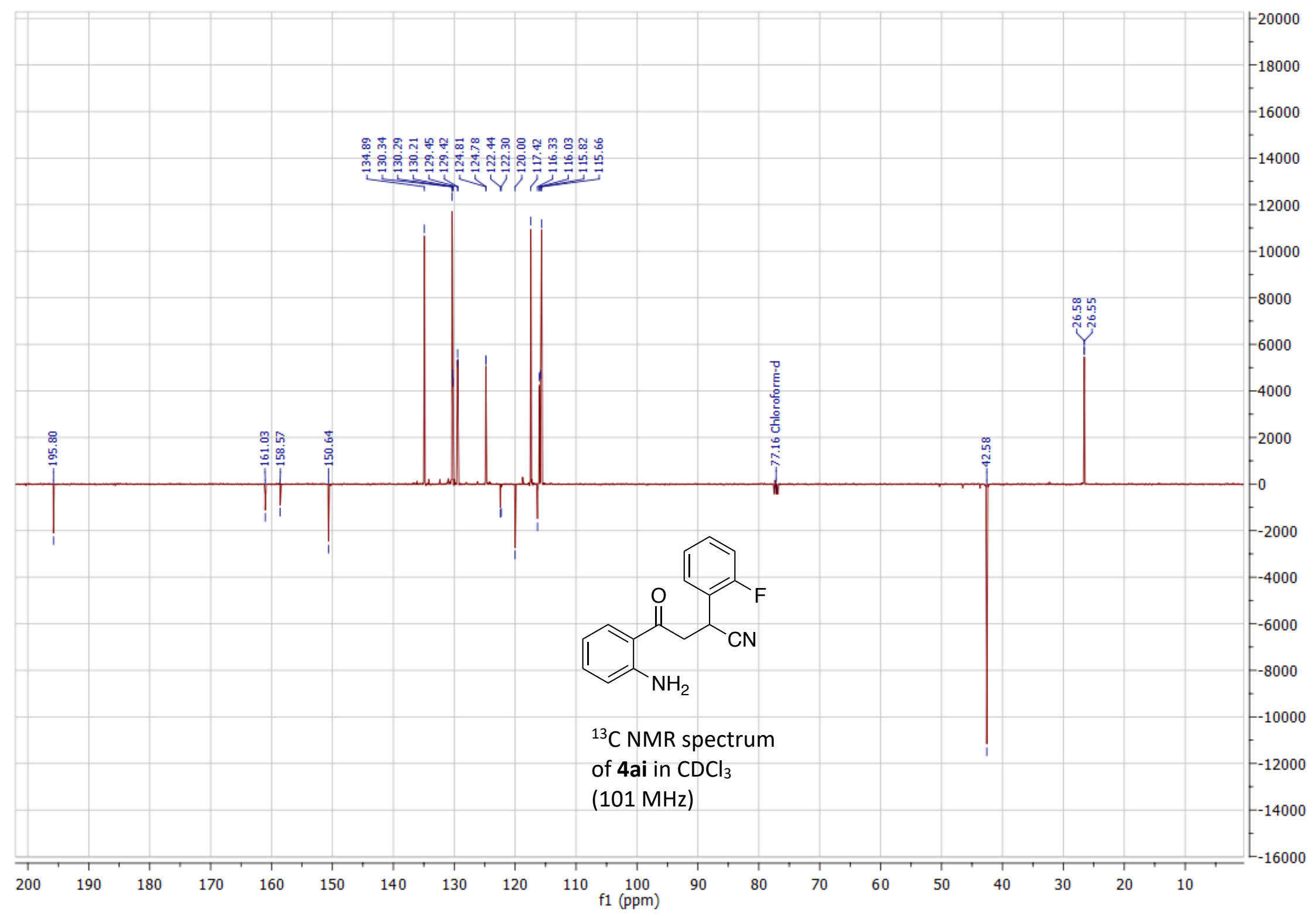




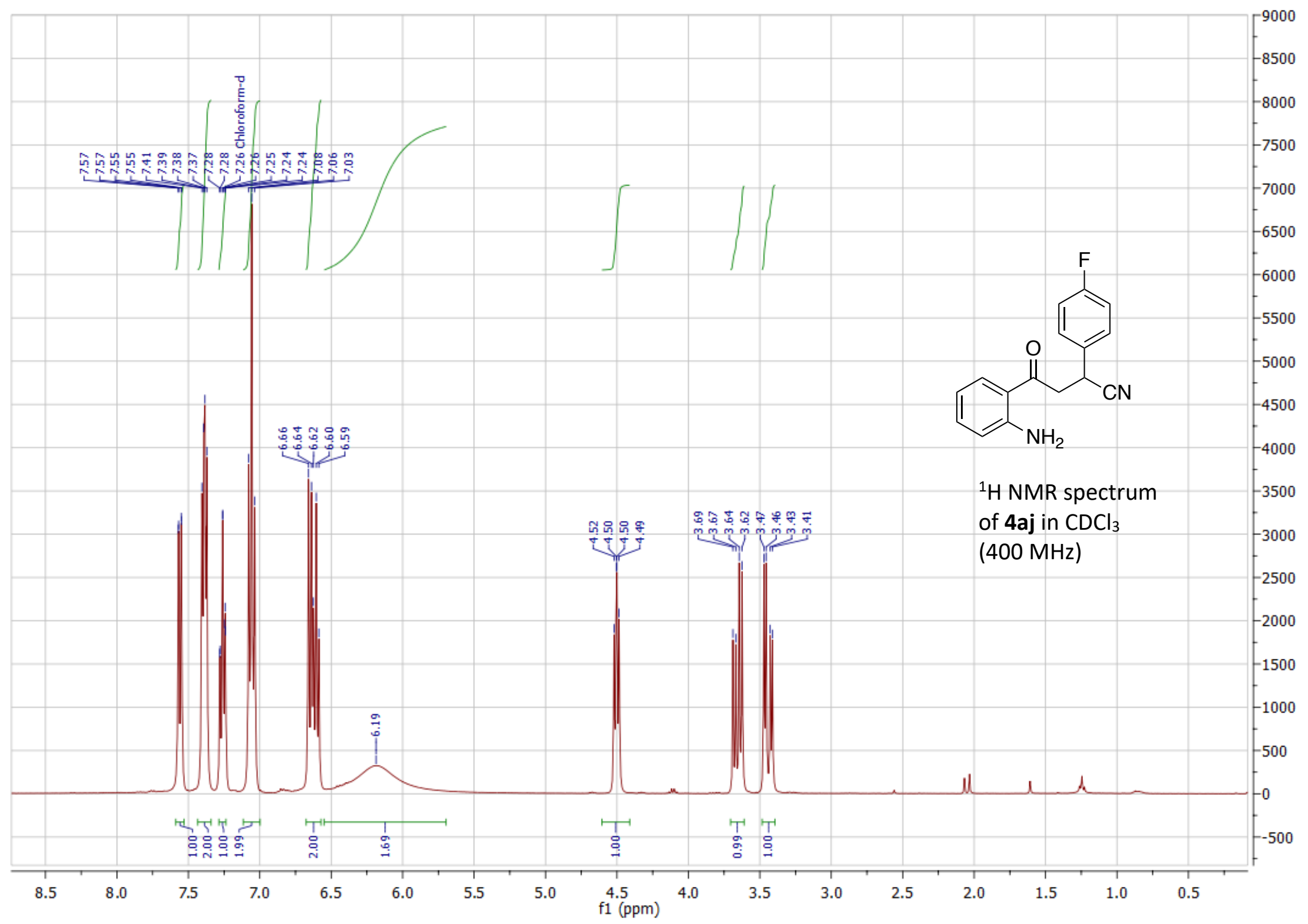




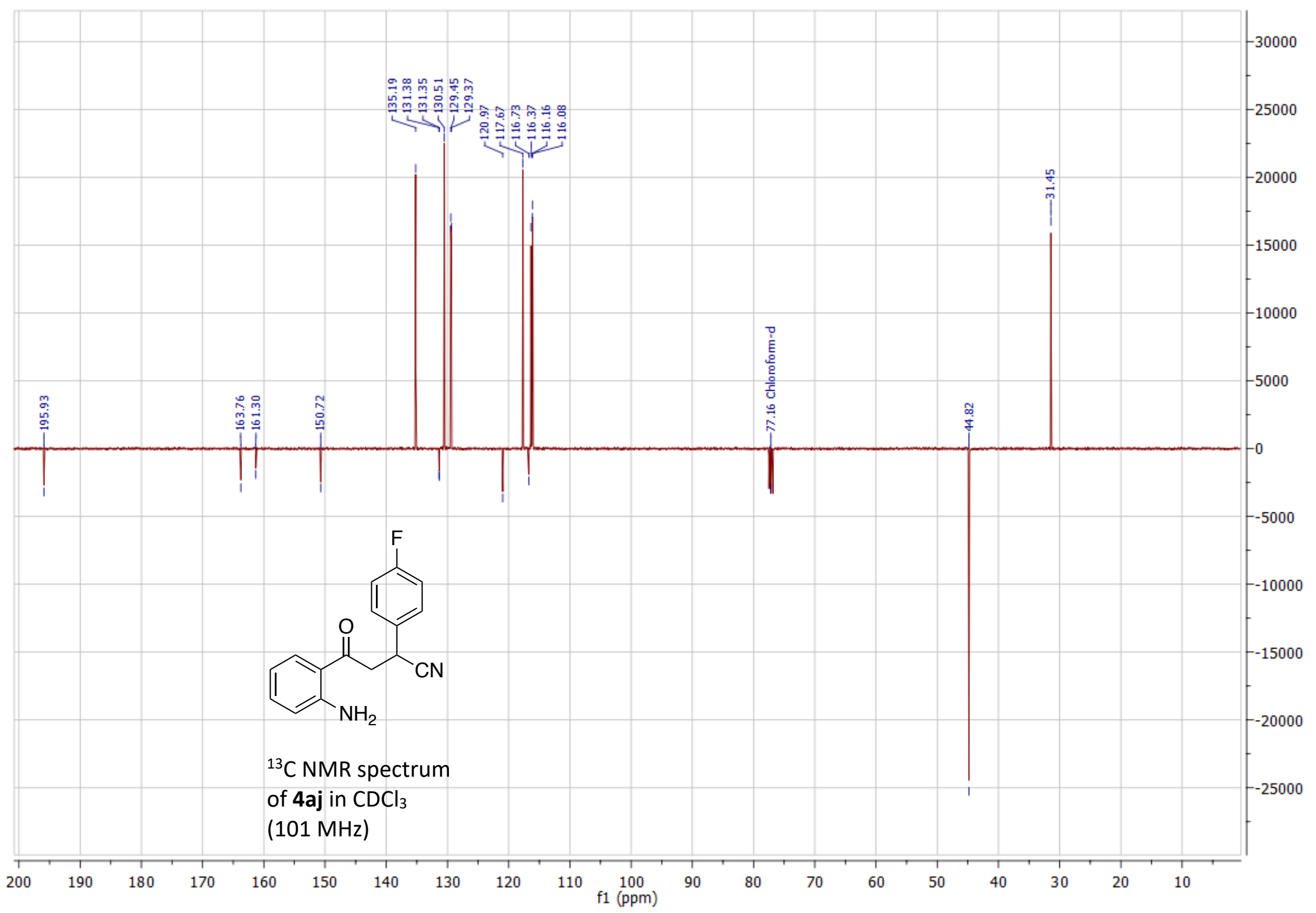




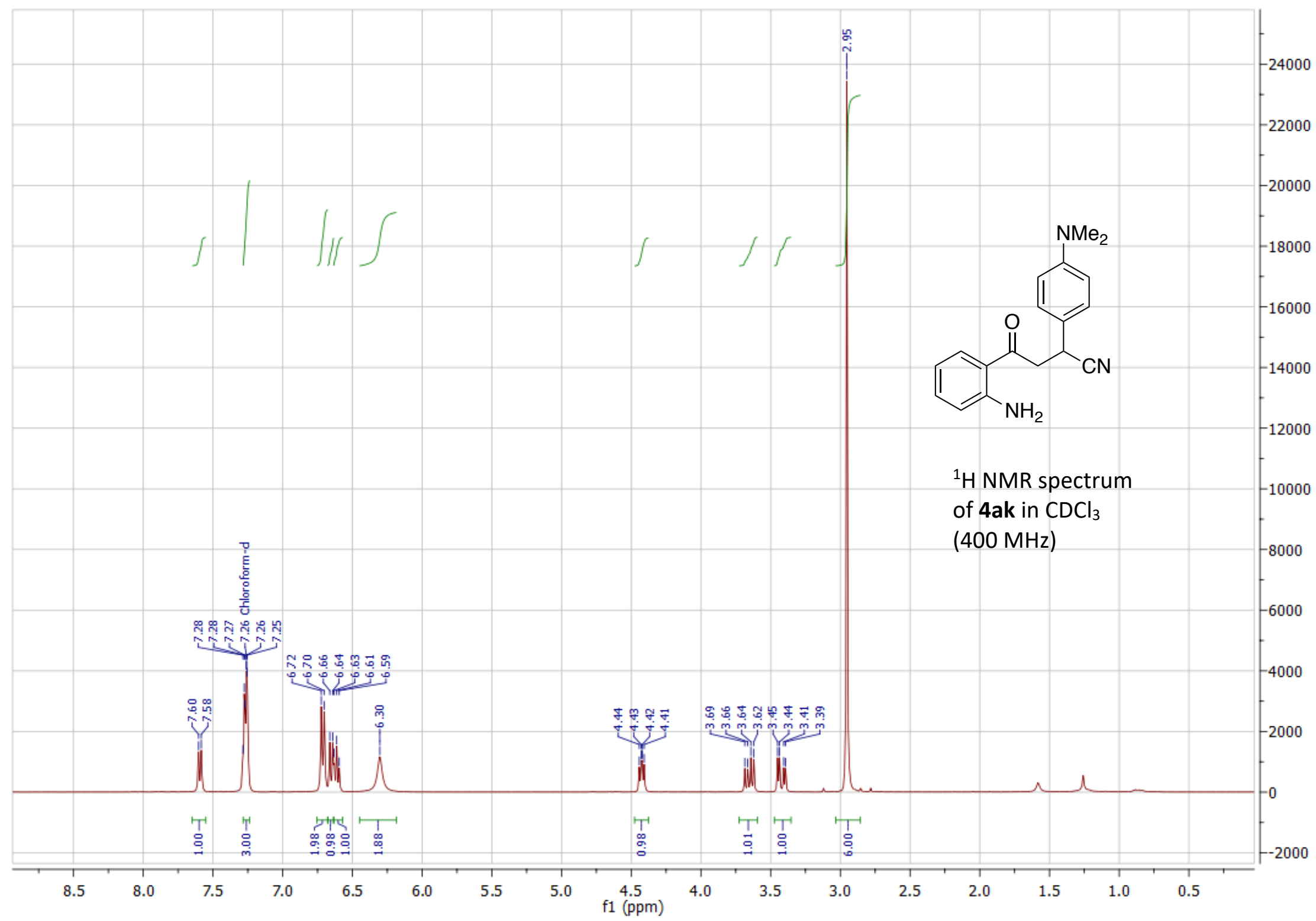




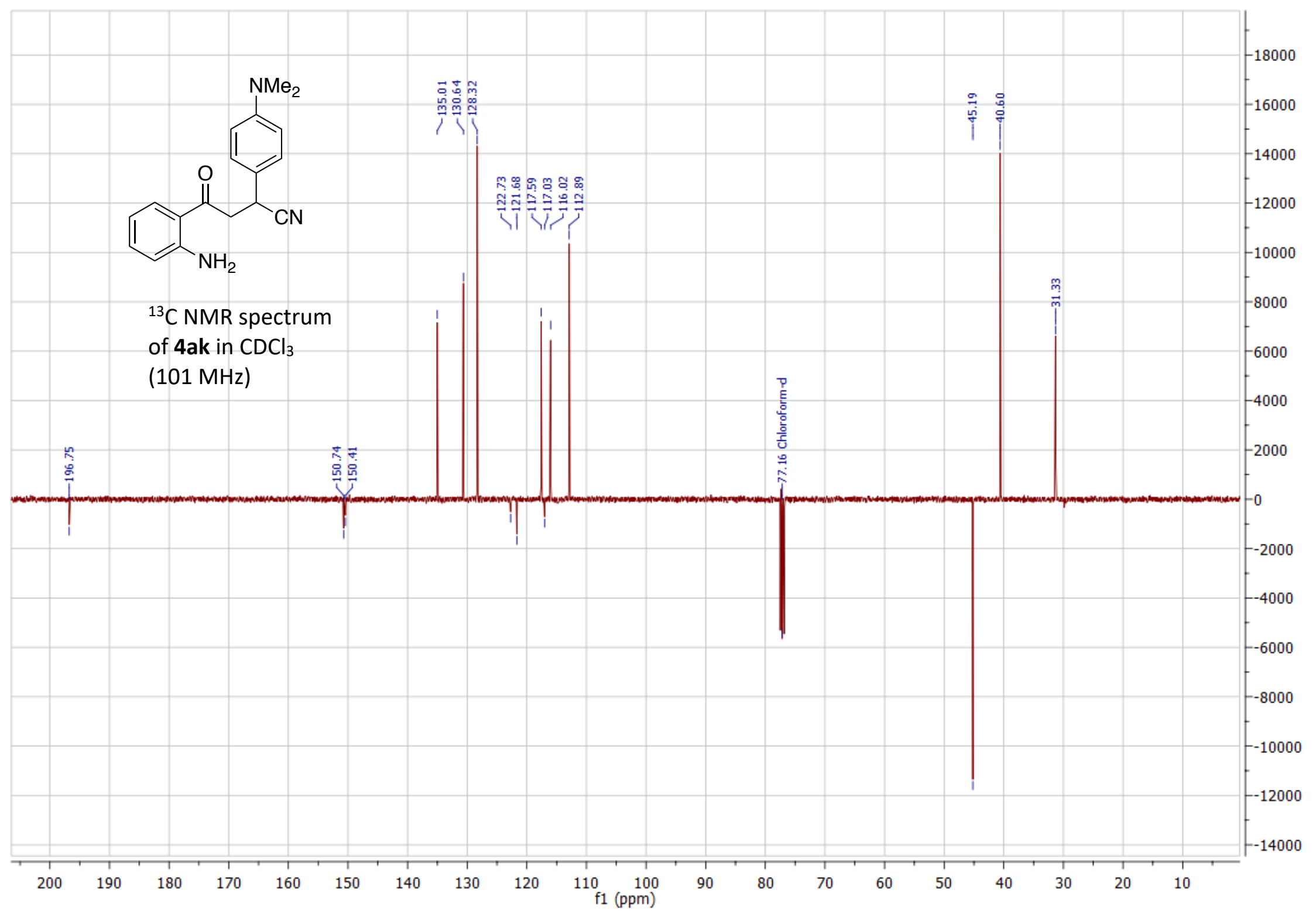




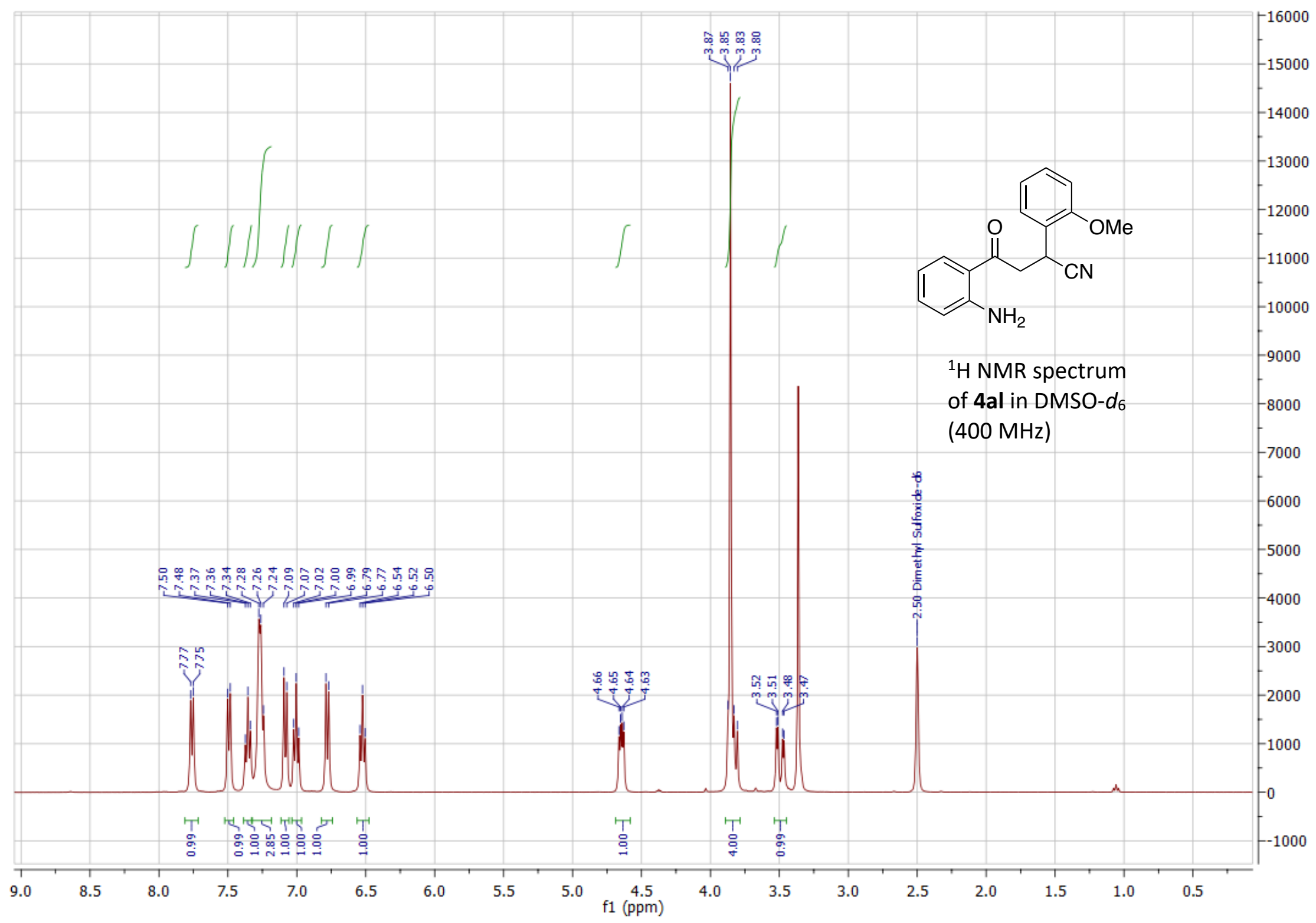




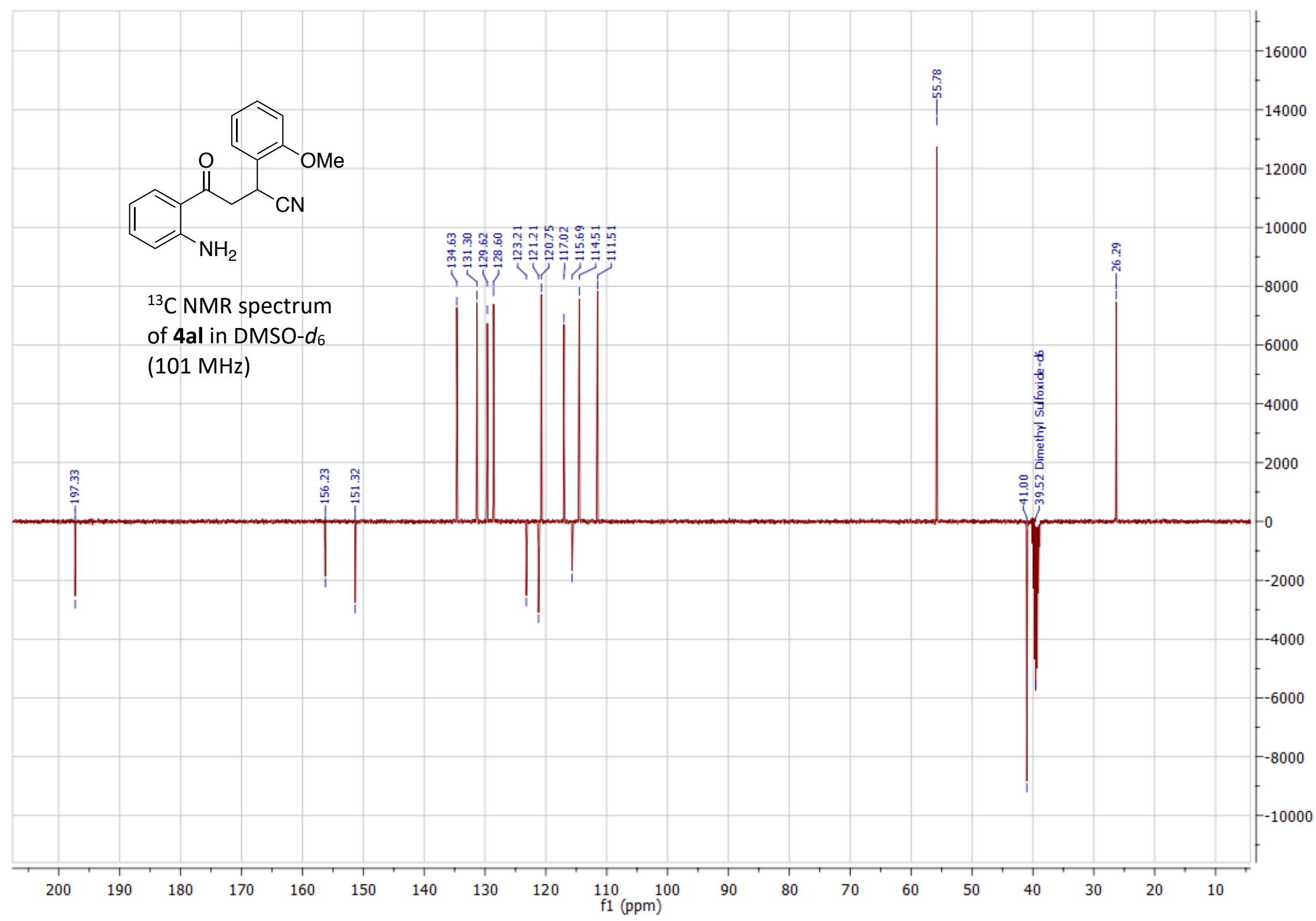




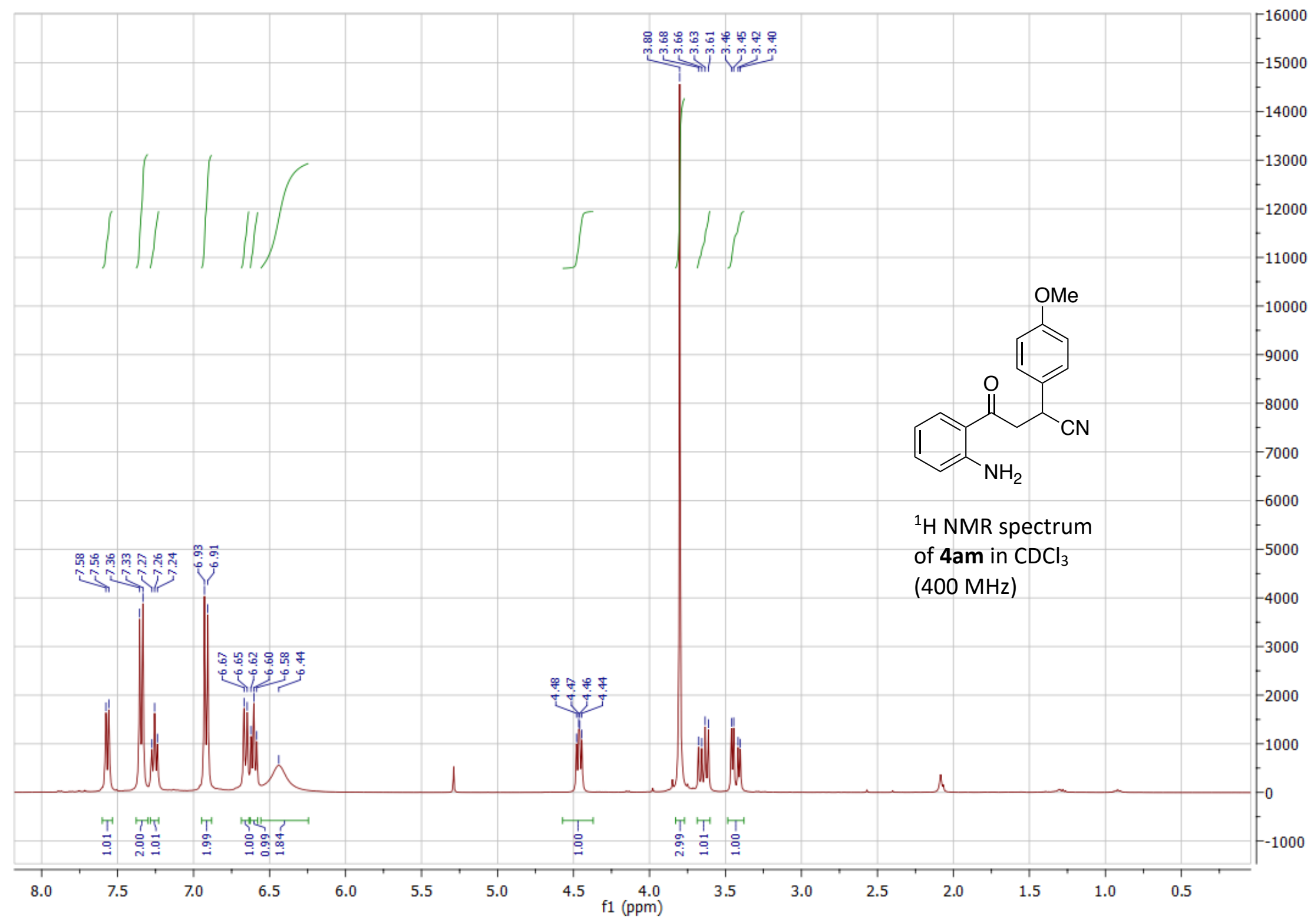




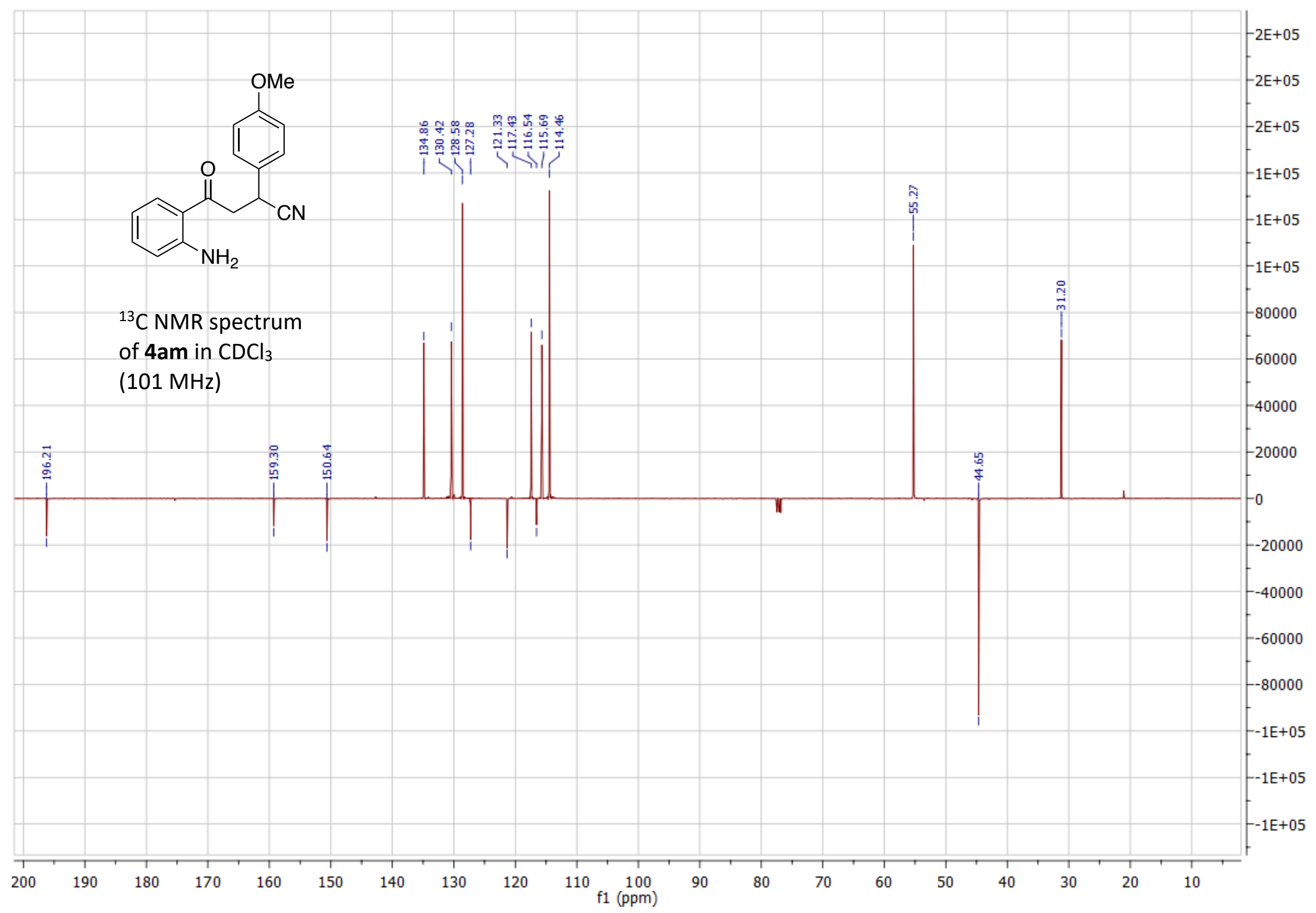




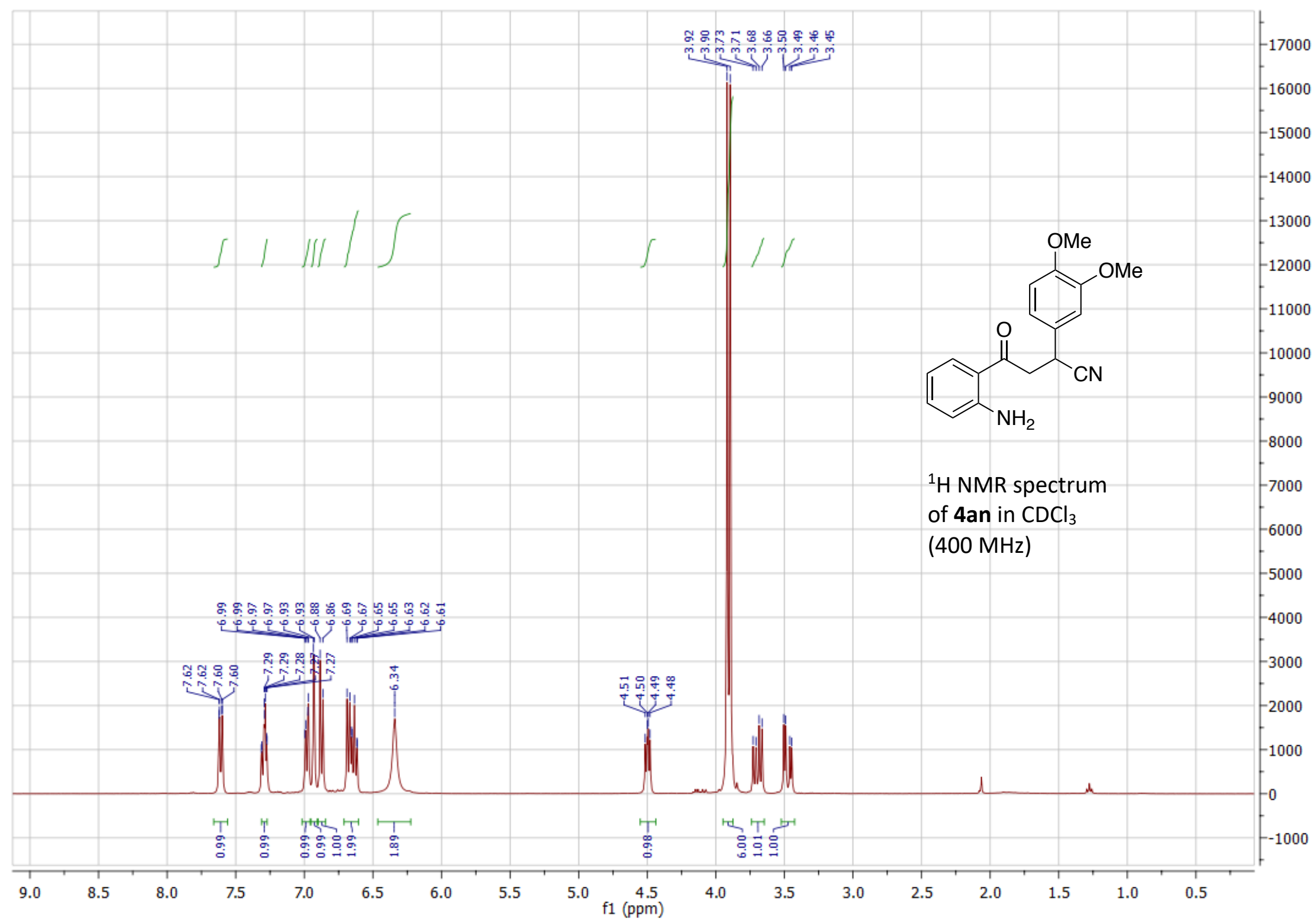




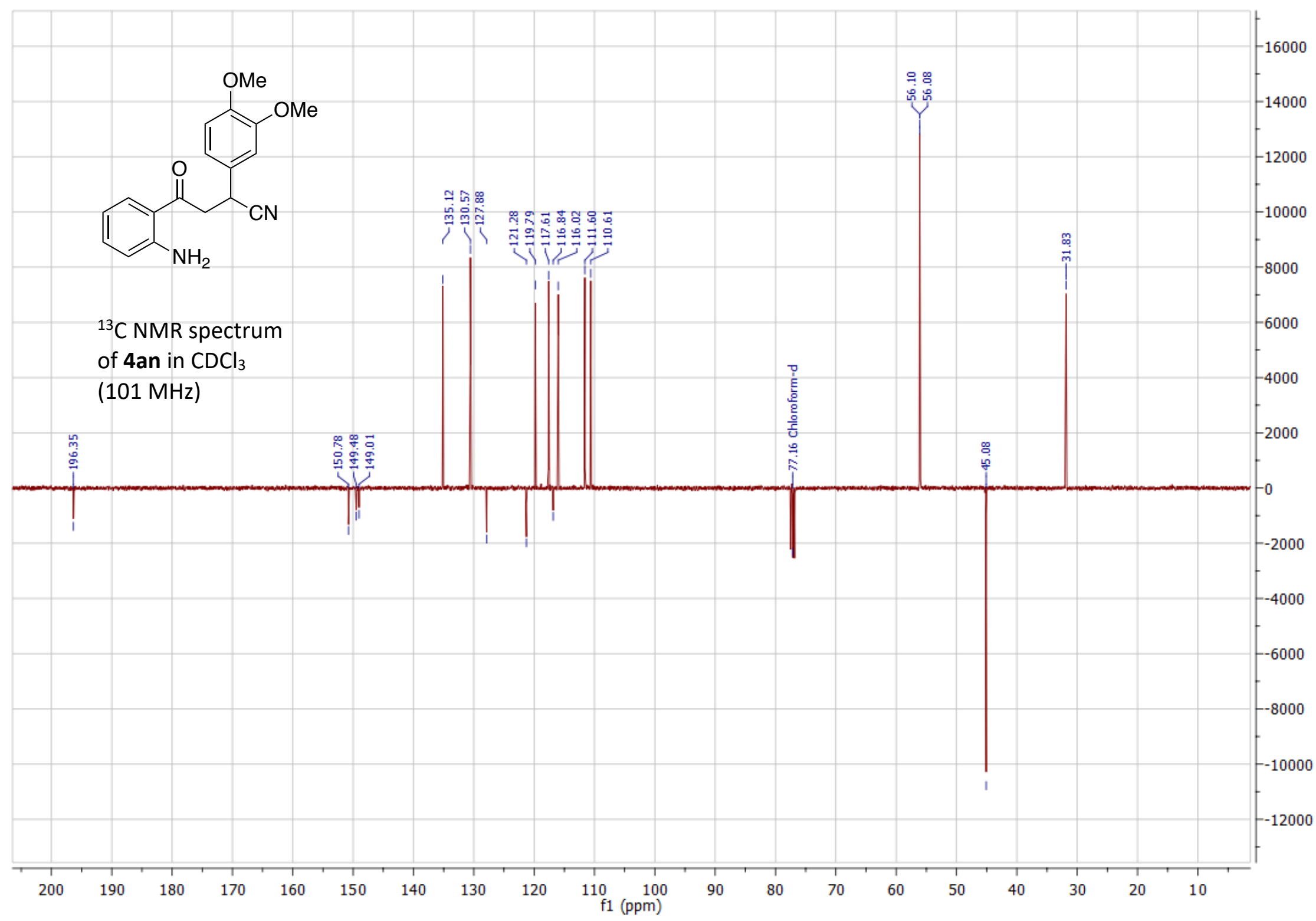




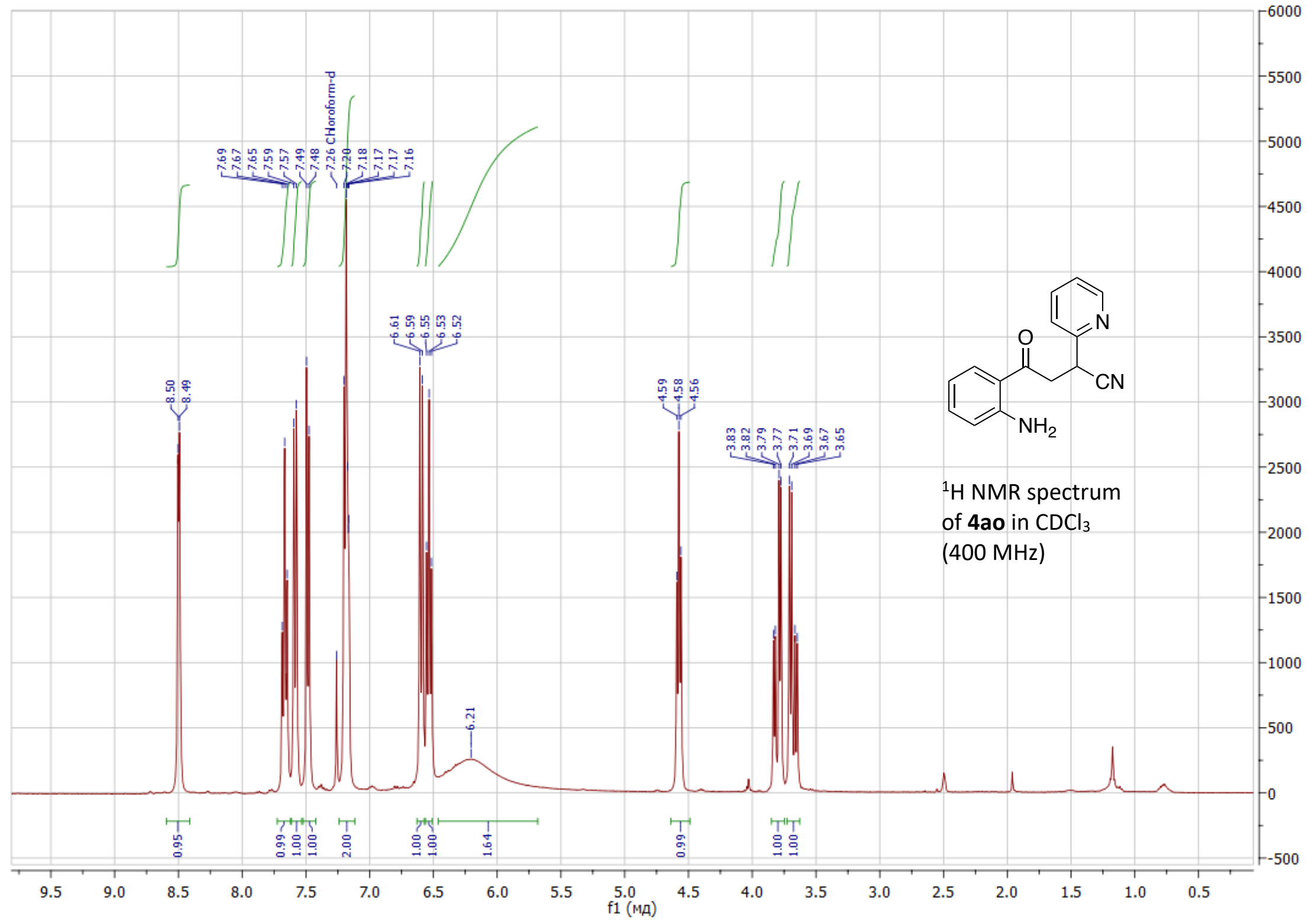




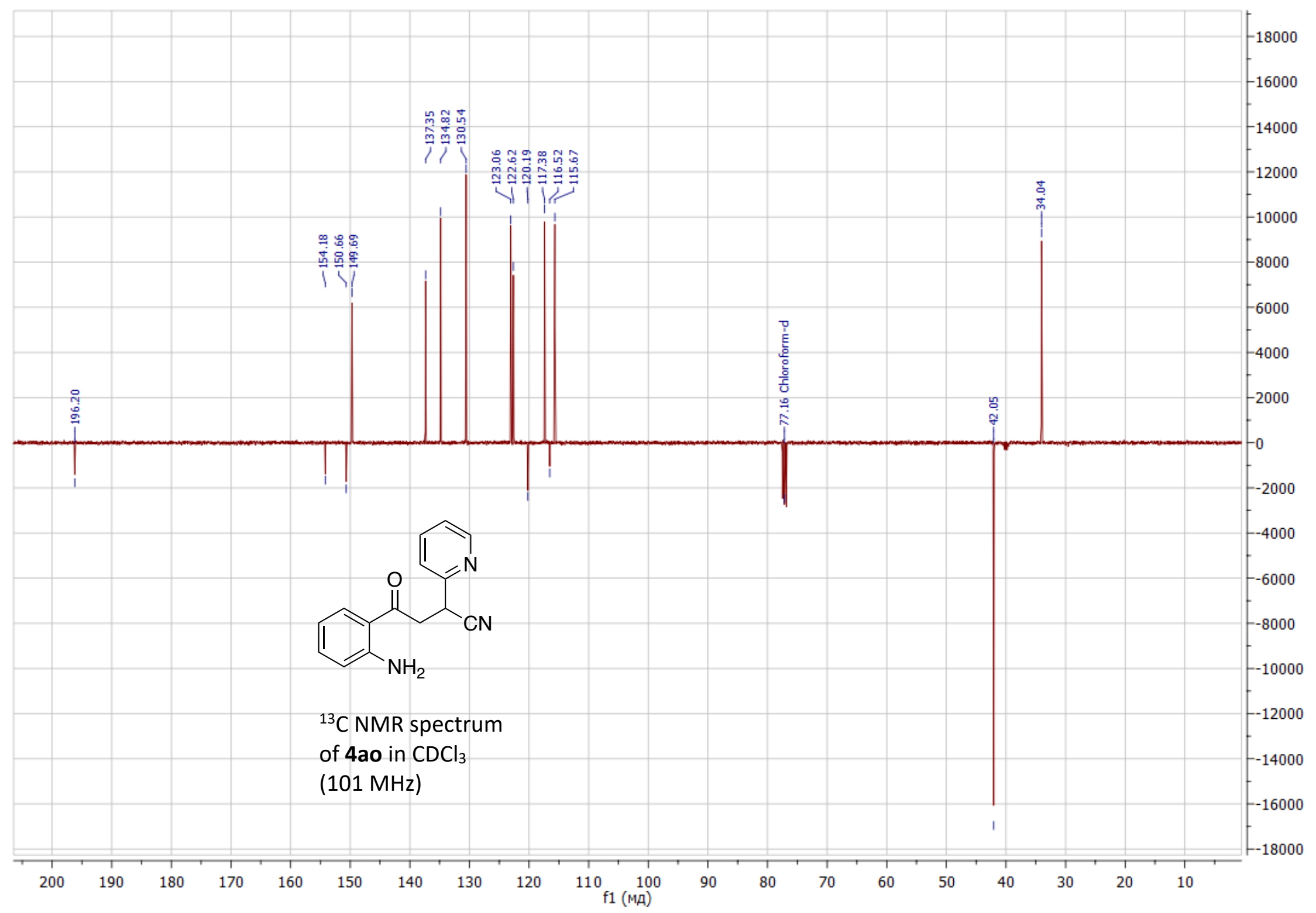




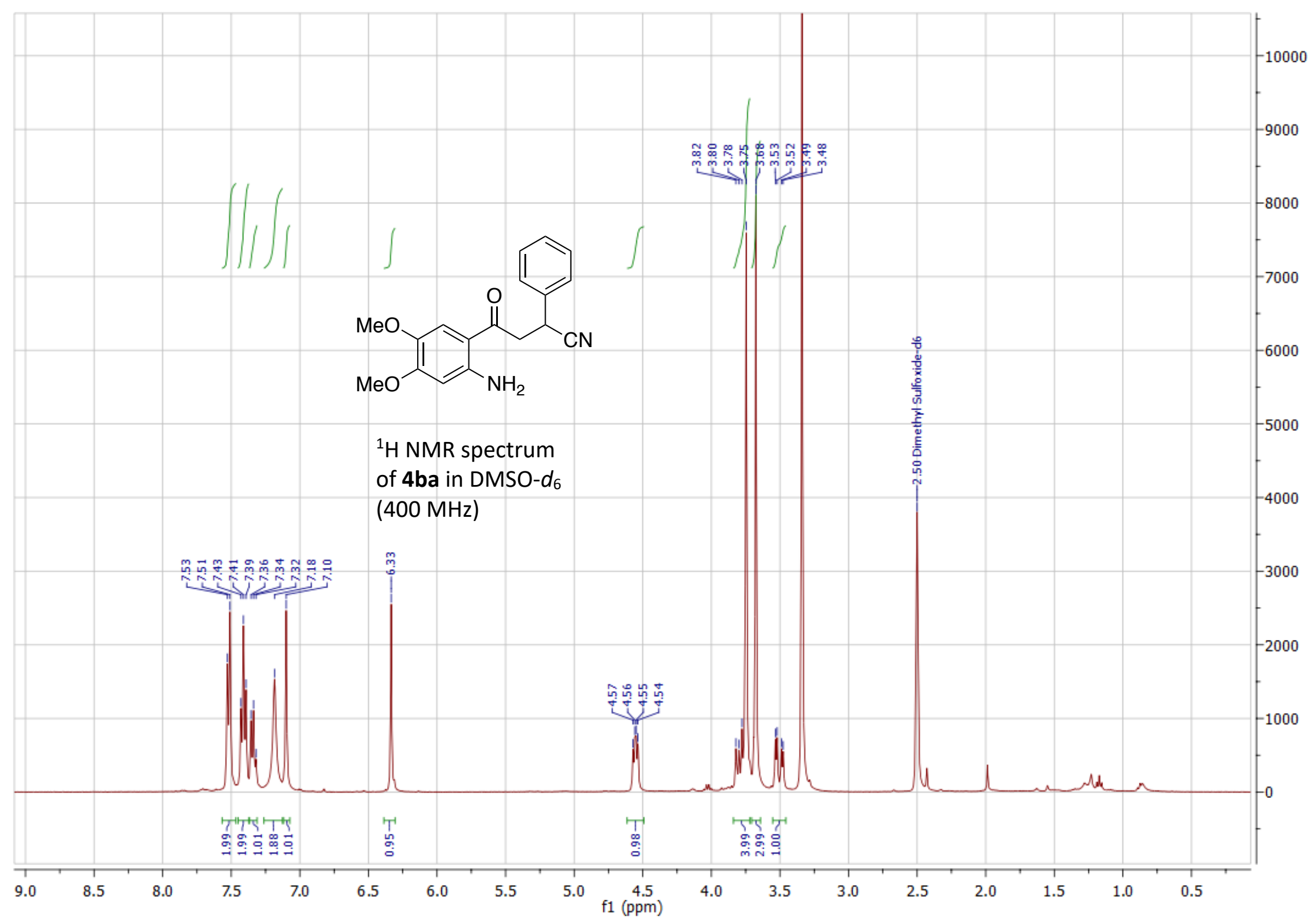




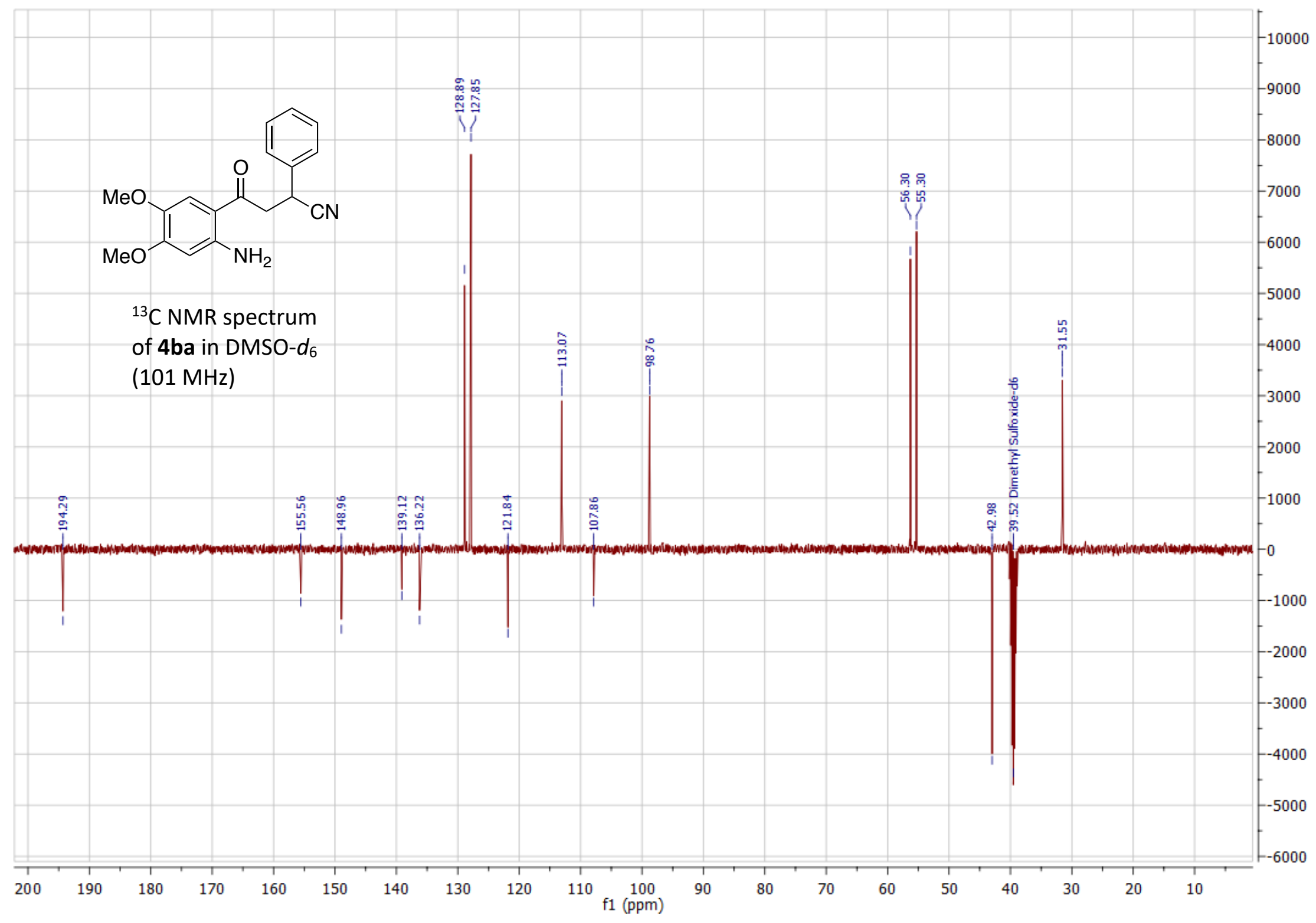




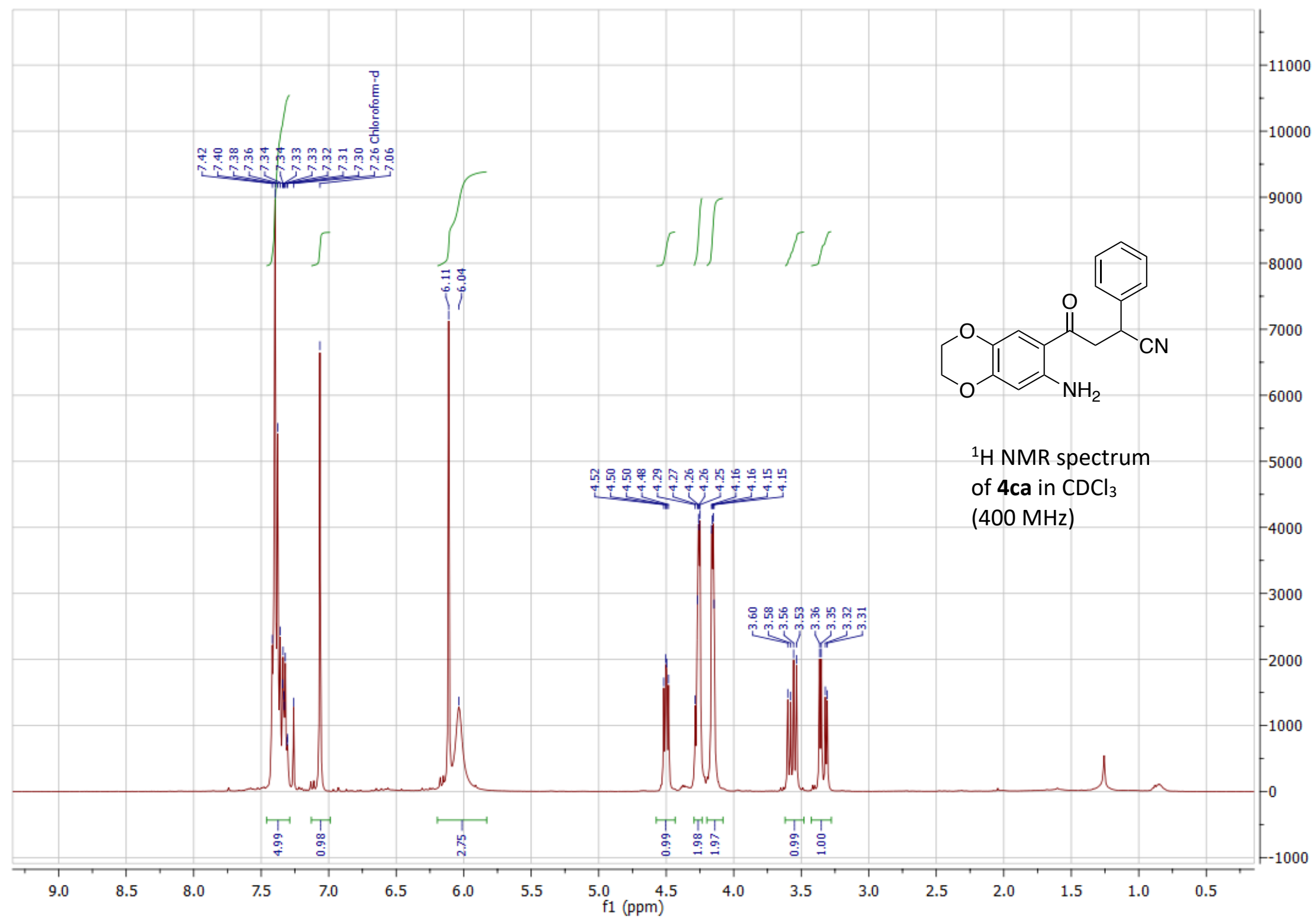




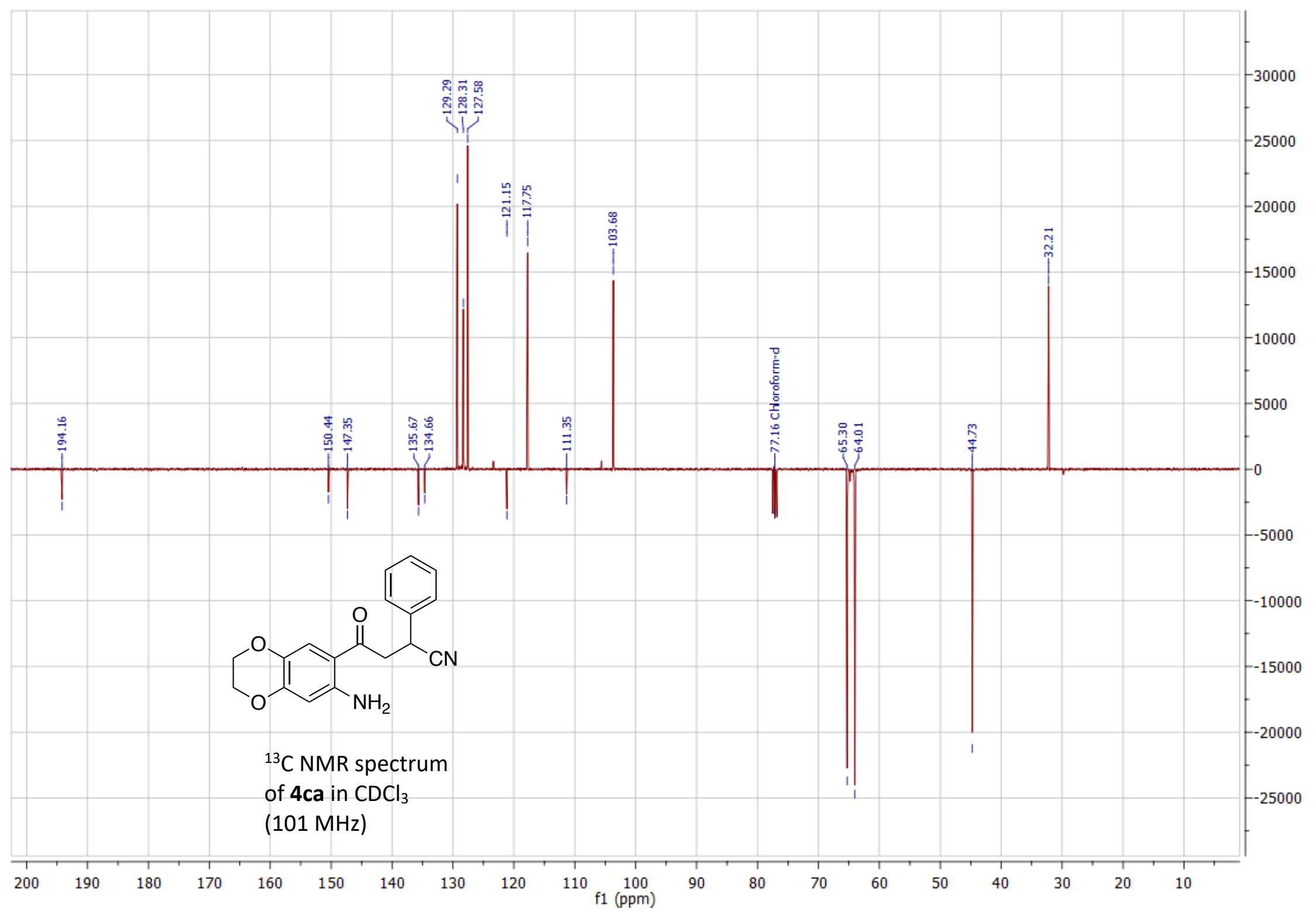




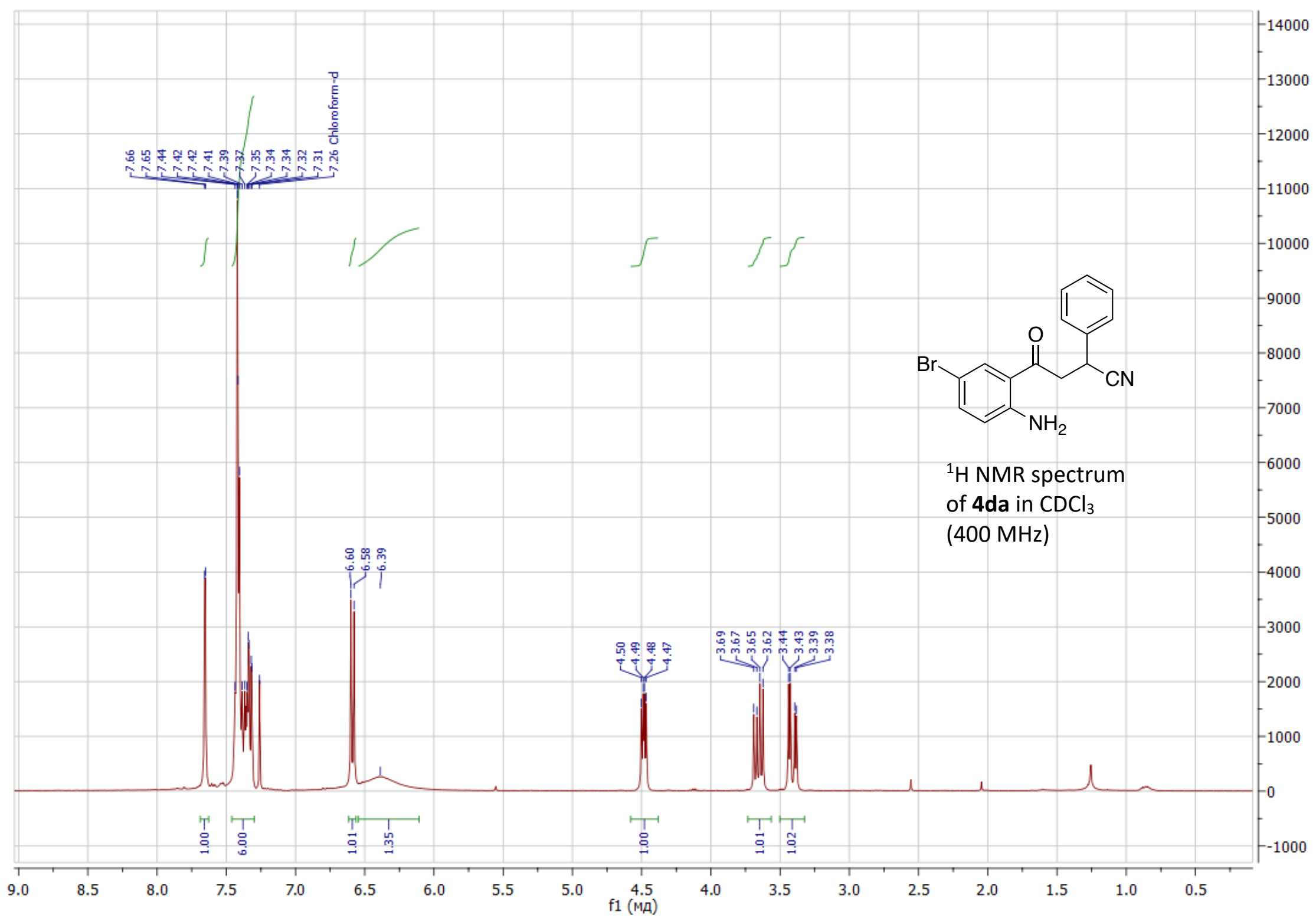




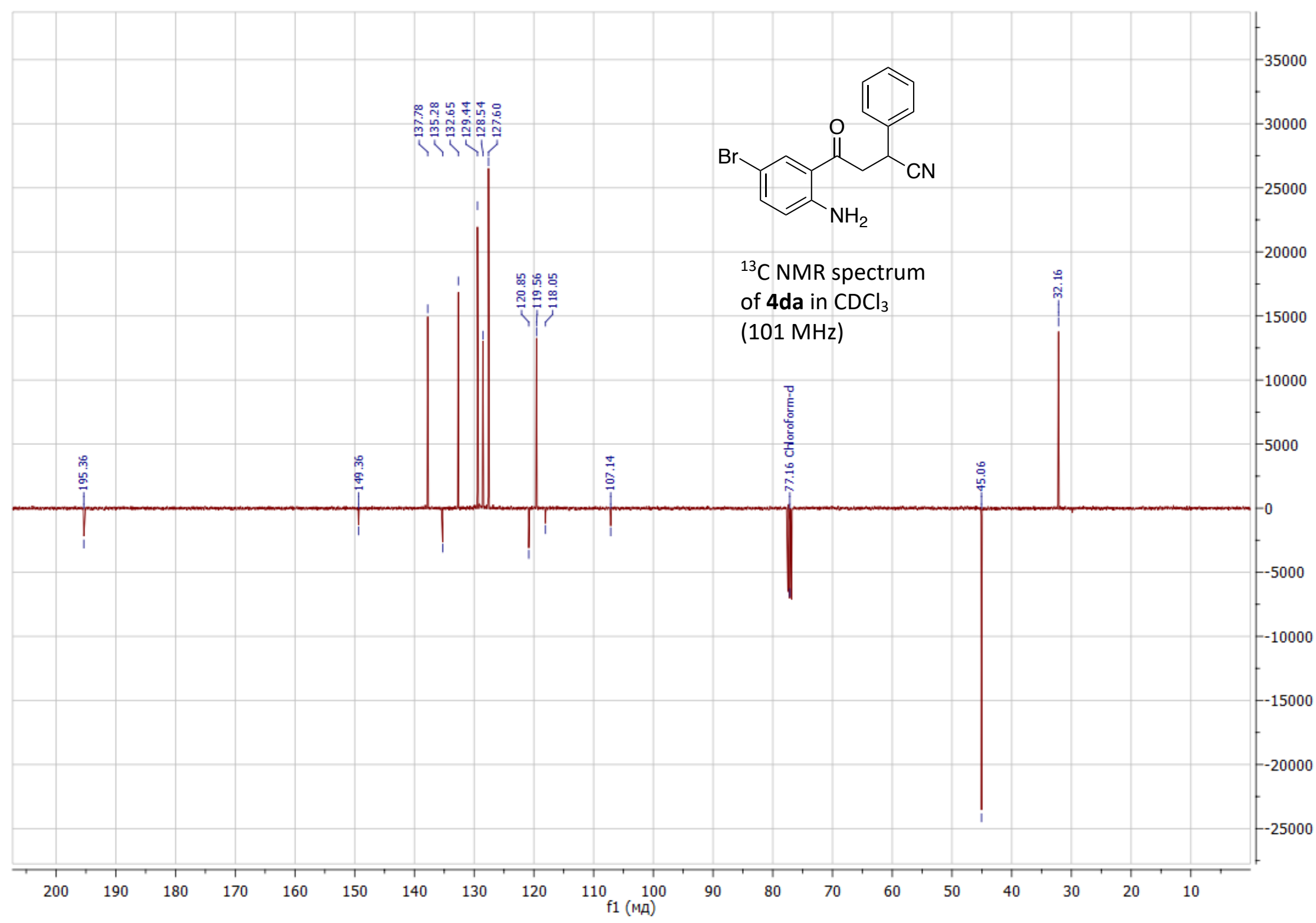


${ }^{1} \mathrm{H}$ and ${ }^{13} \mathrm{C}$ Spectral Charts of 4-(2-nitrophenyl)-4-oxo-2-arylbutanenitriles 19

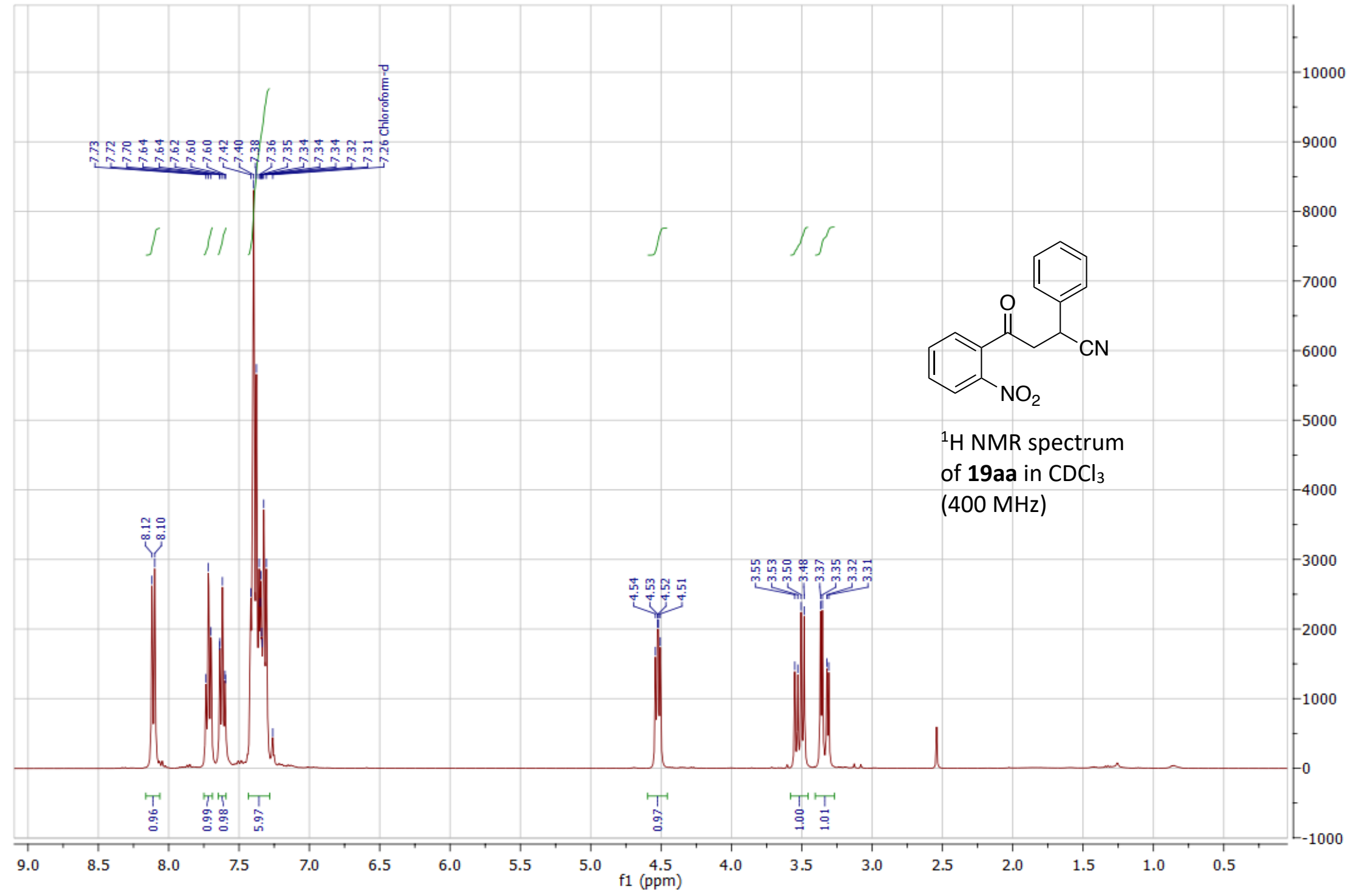




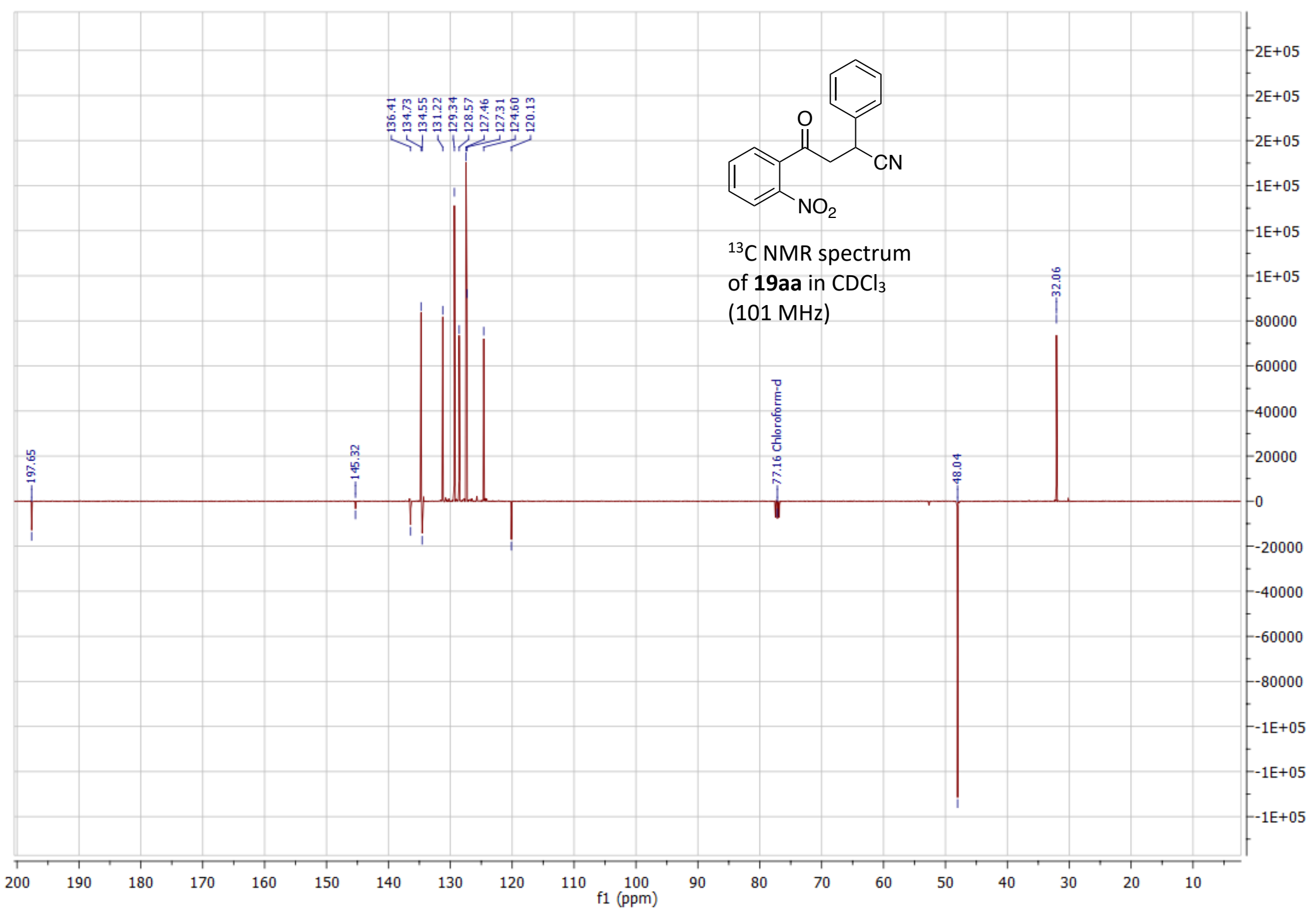




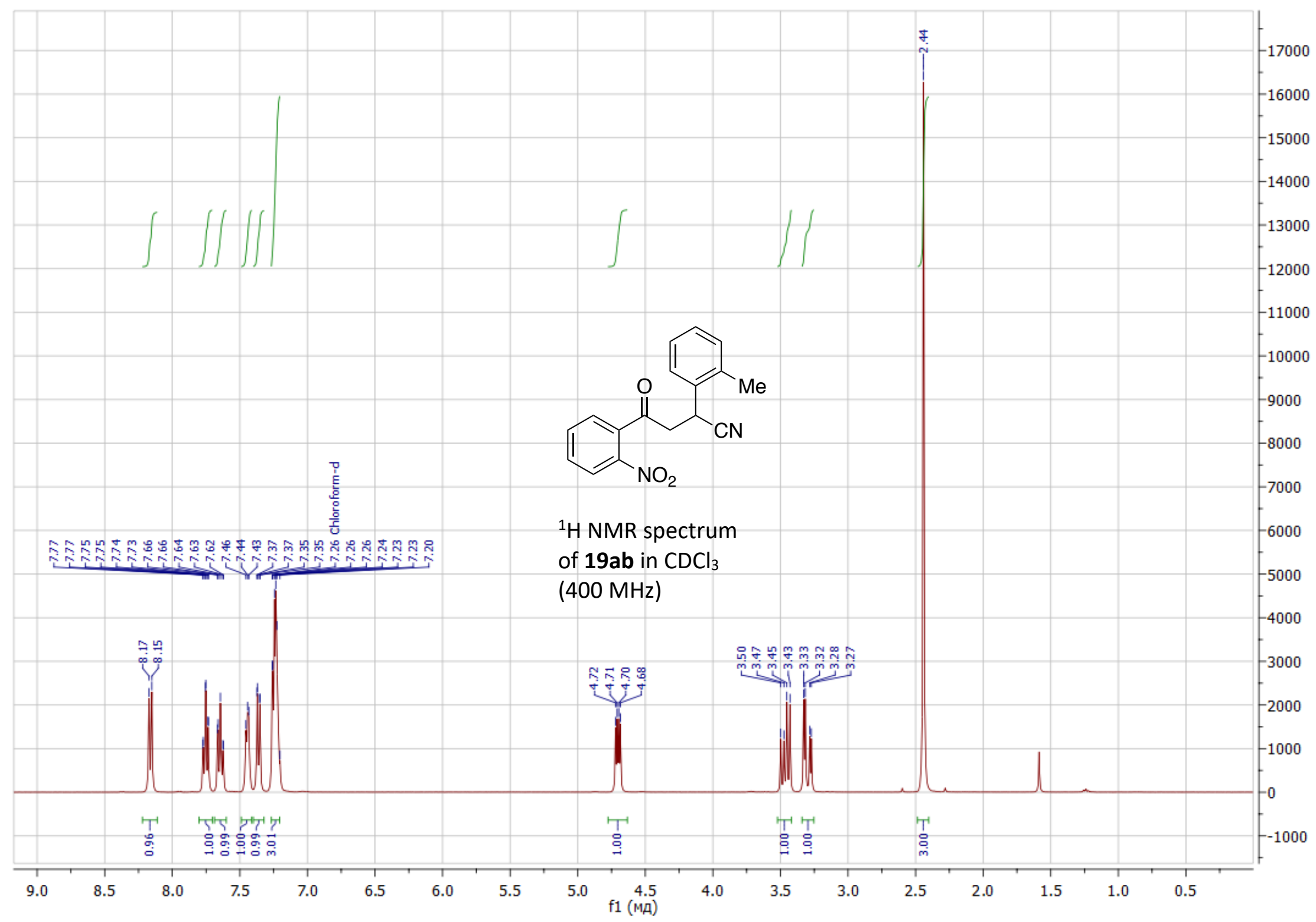




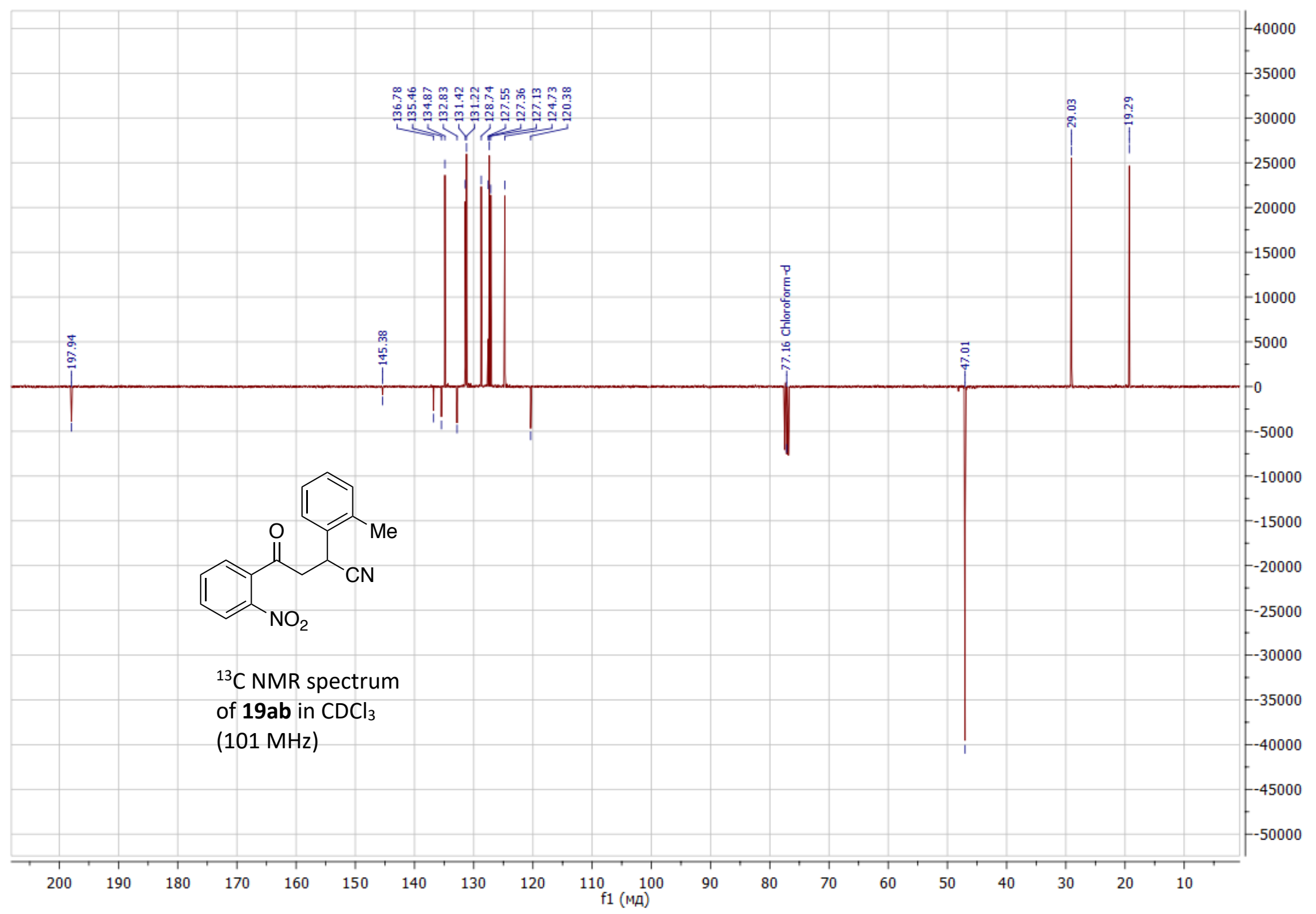




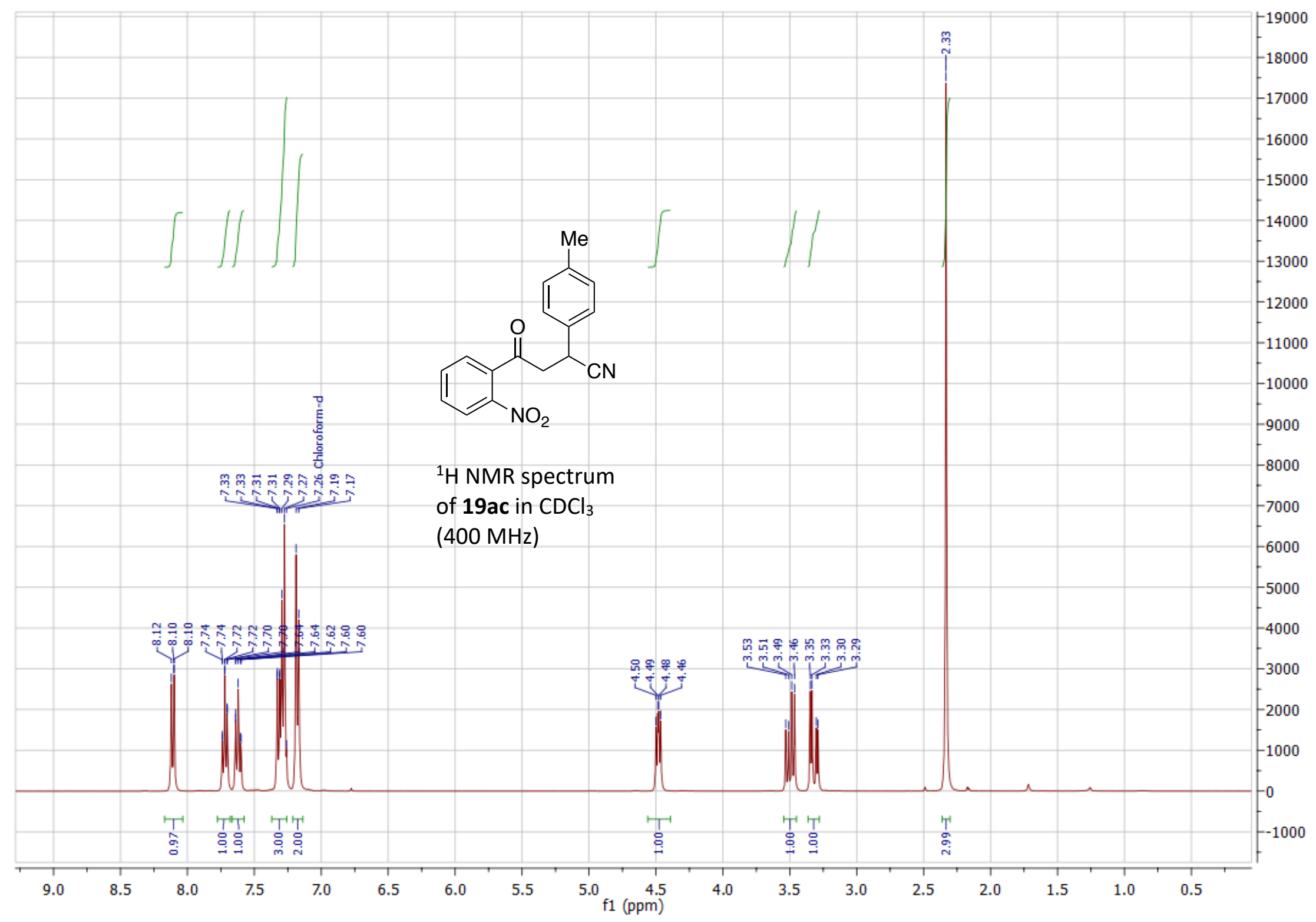




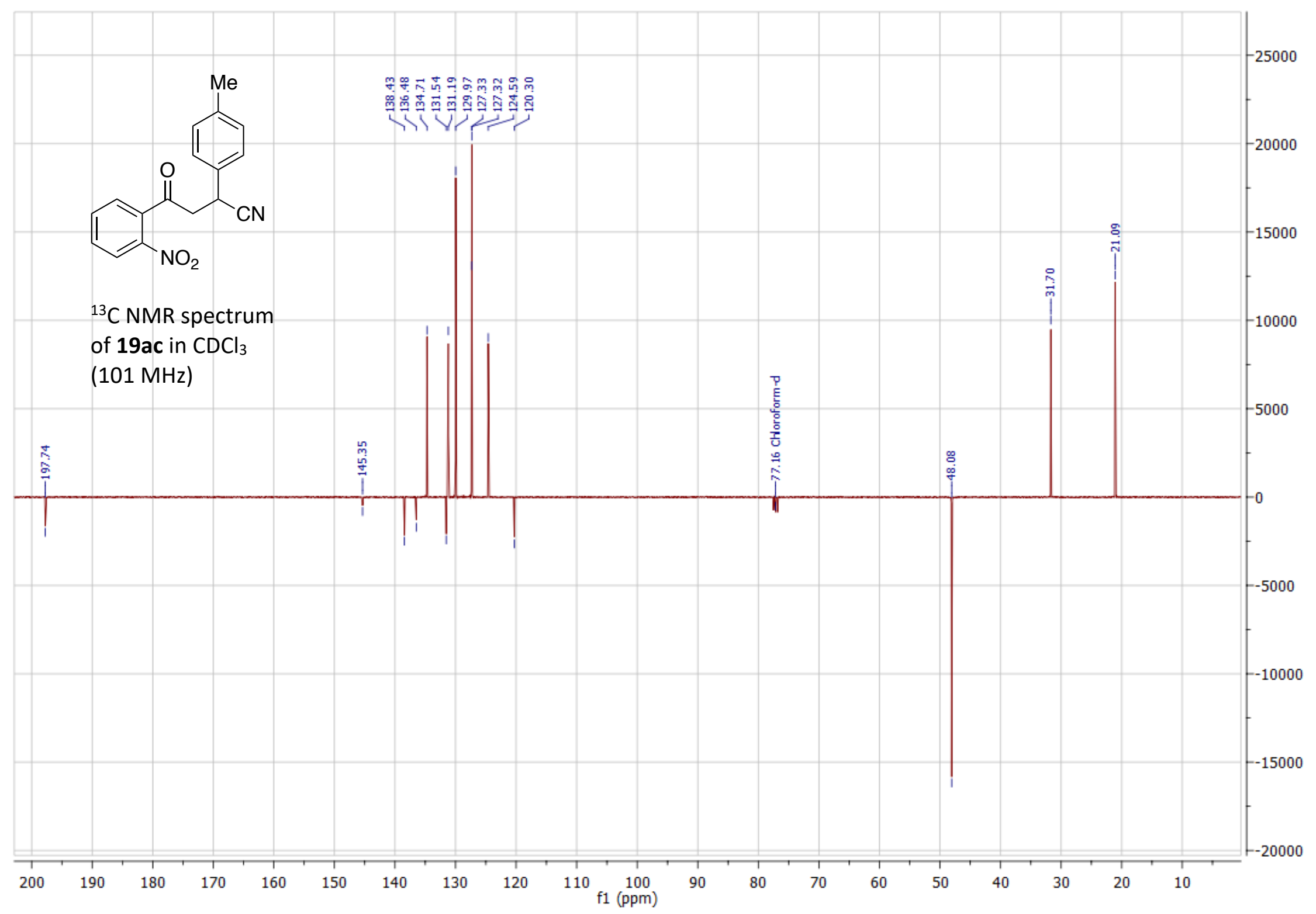




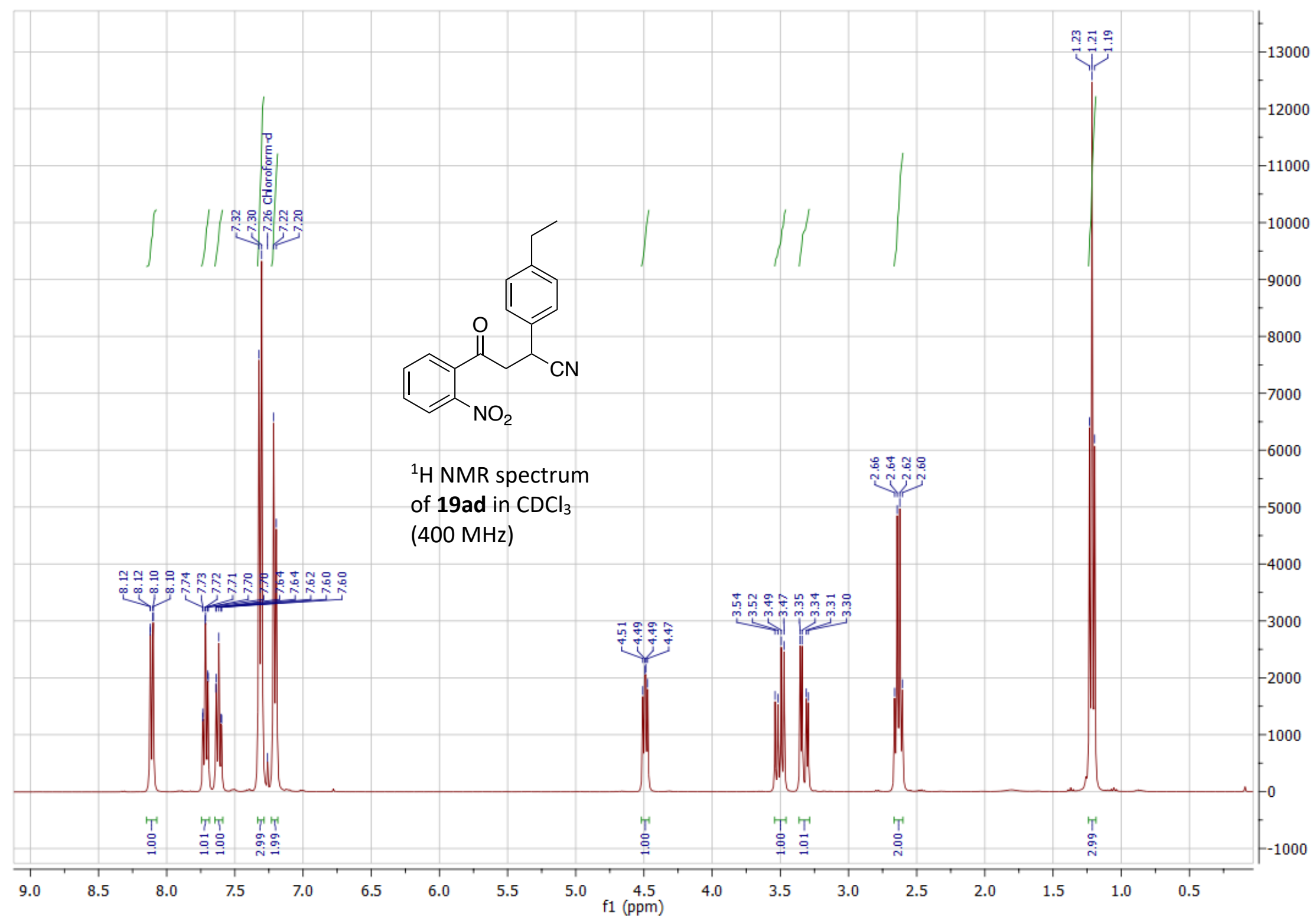




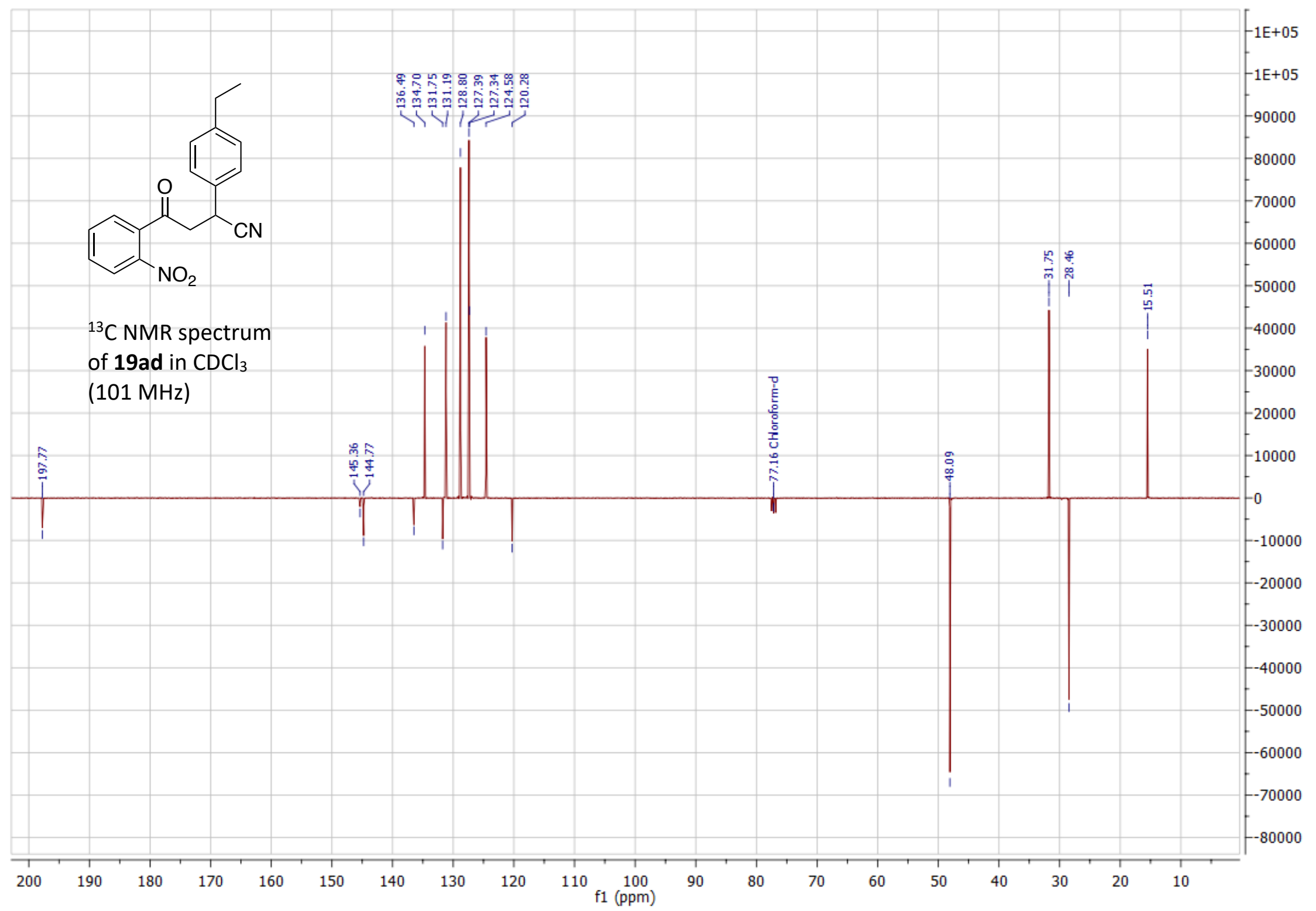




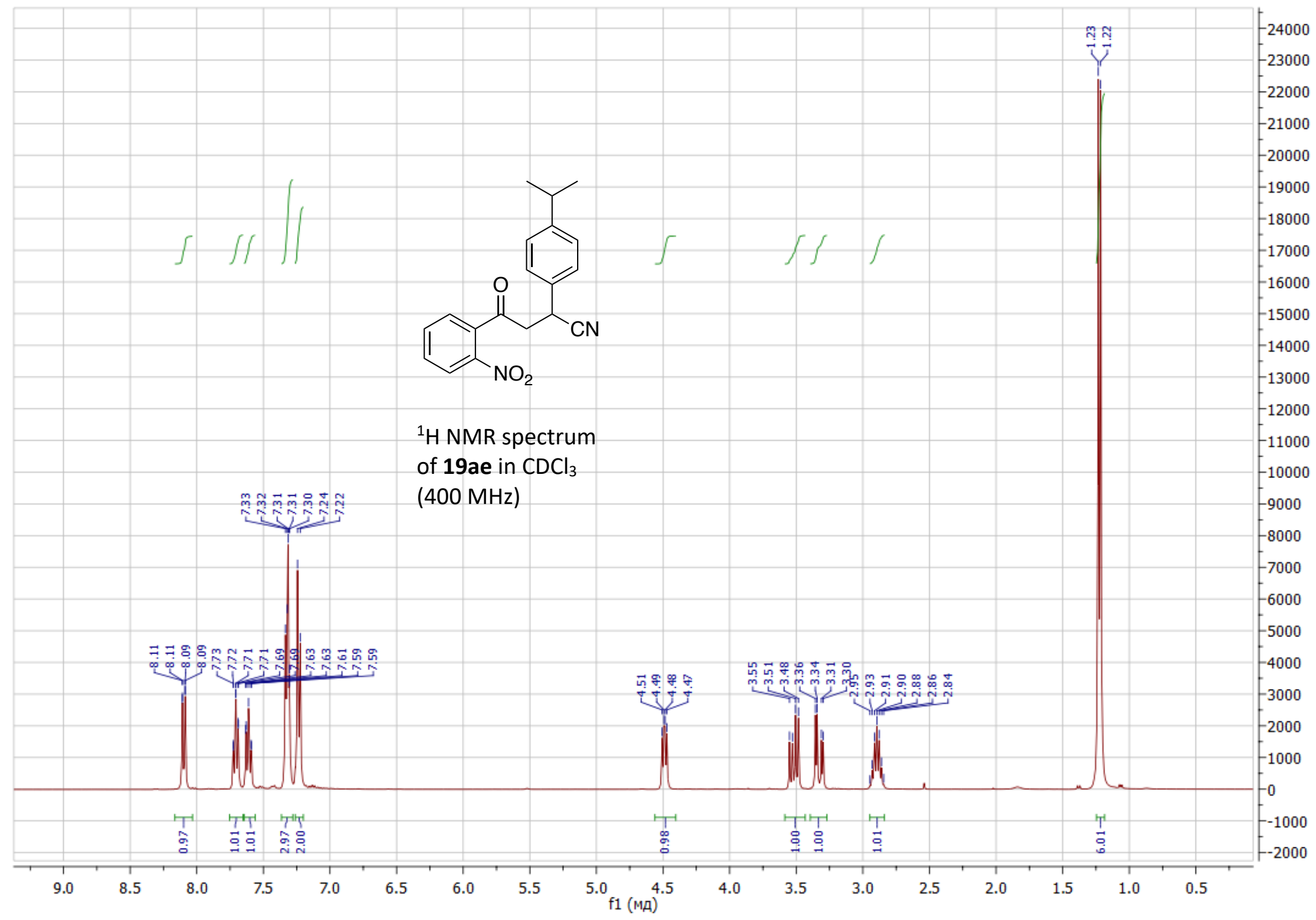




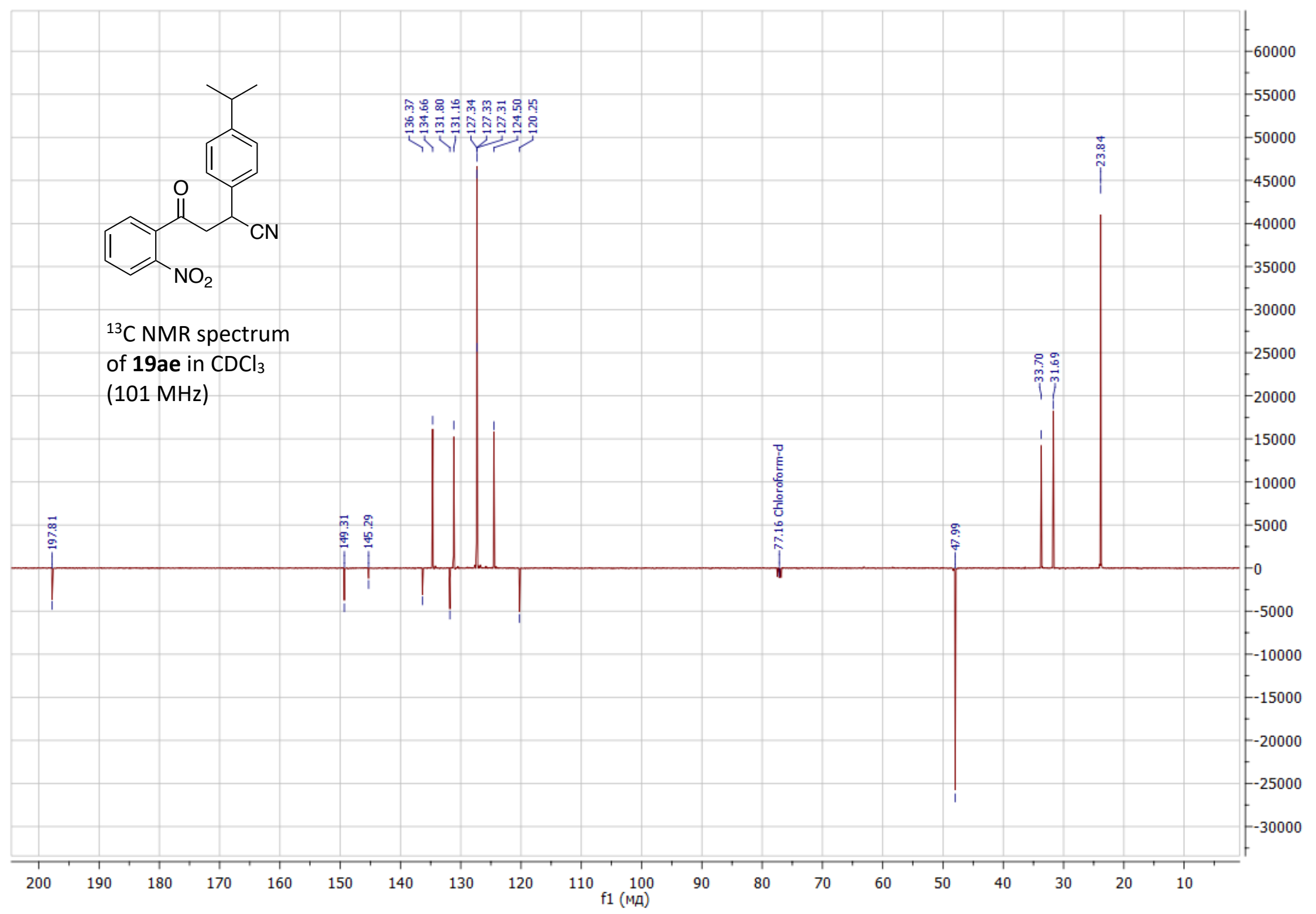




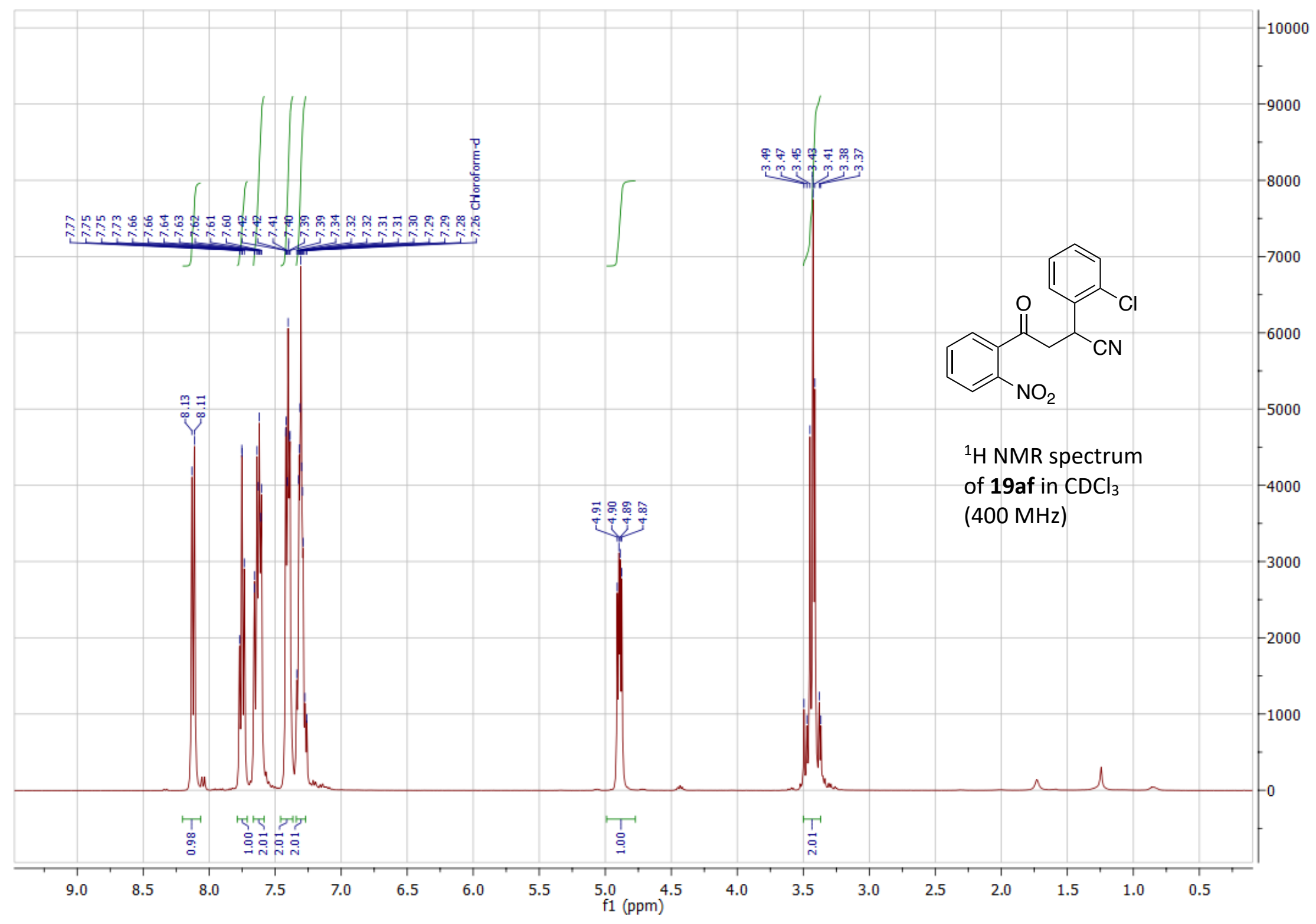




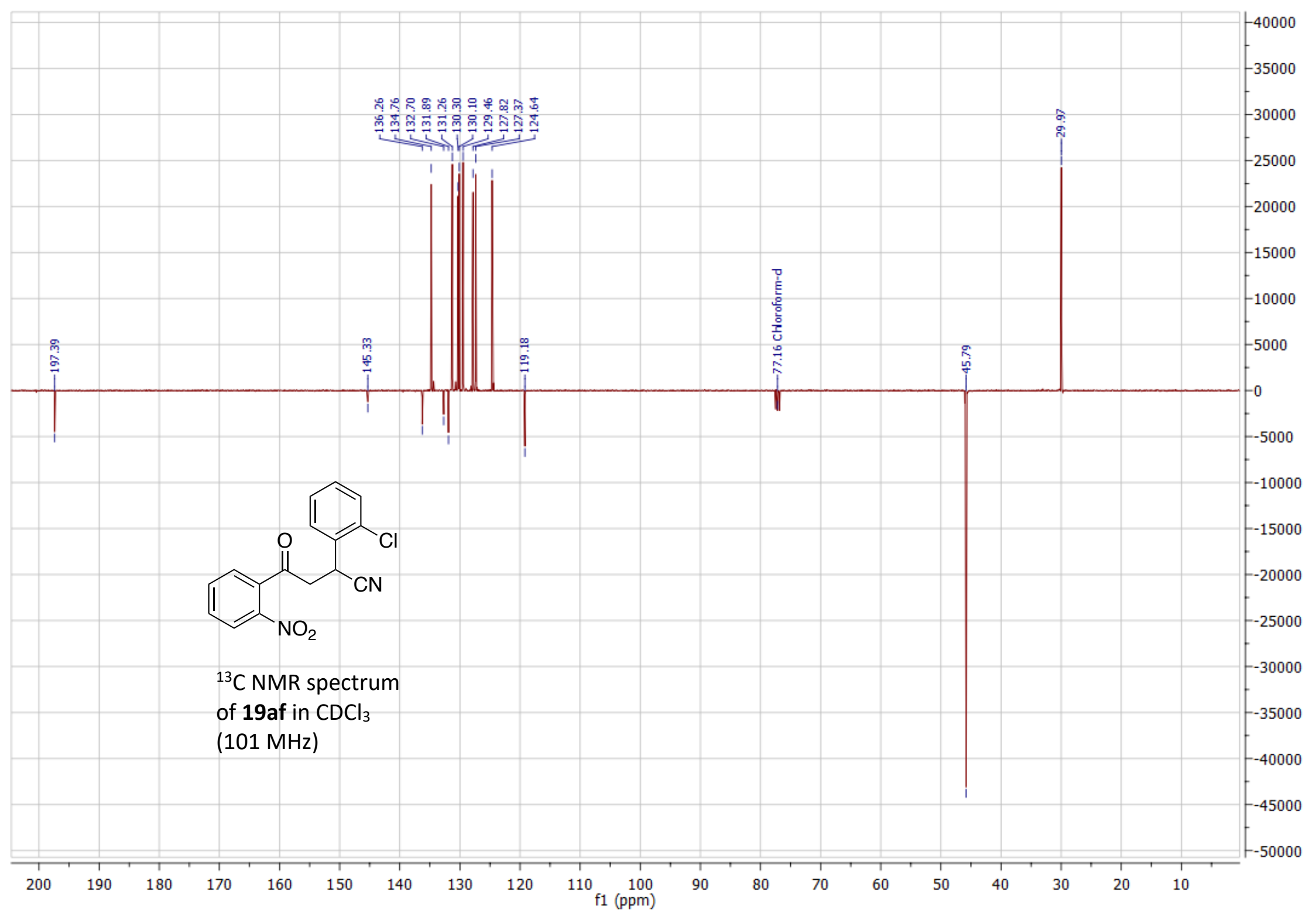




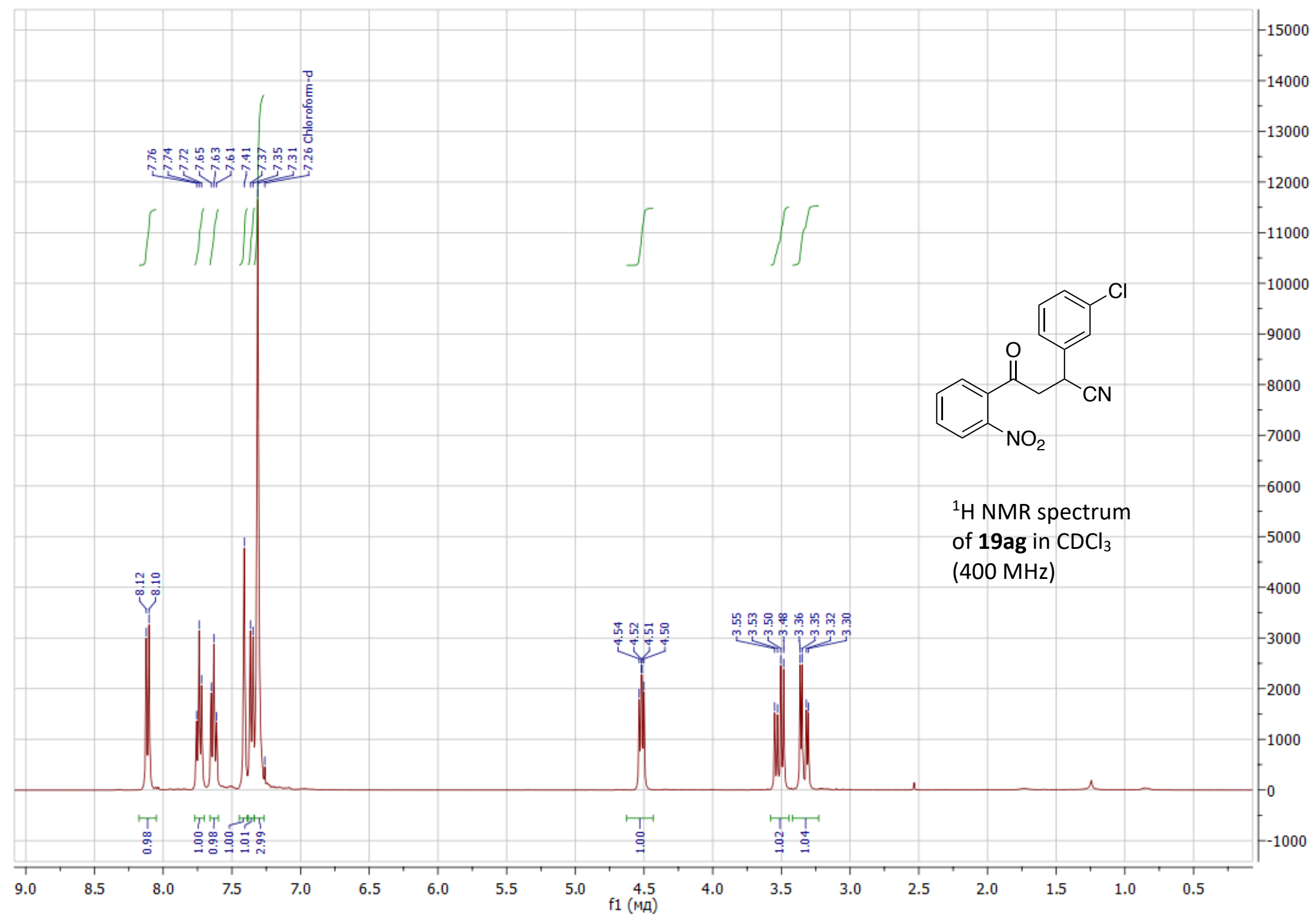




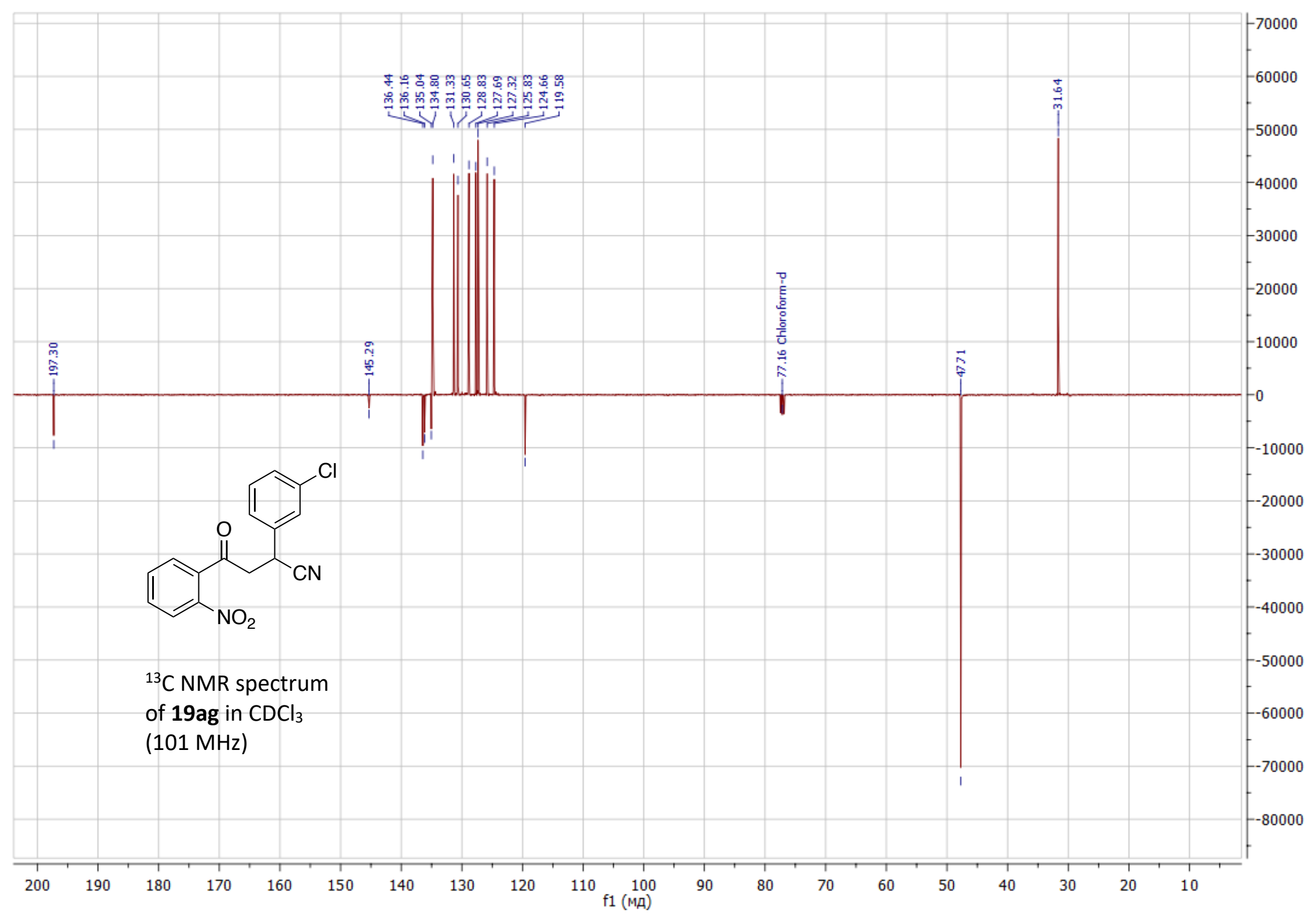




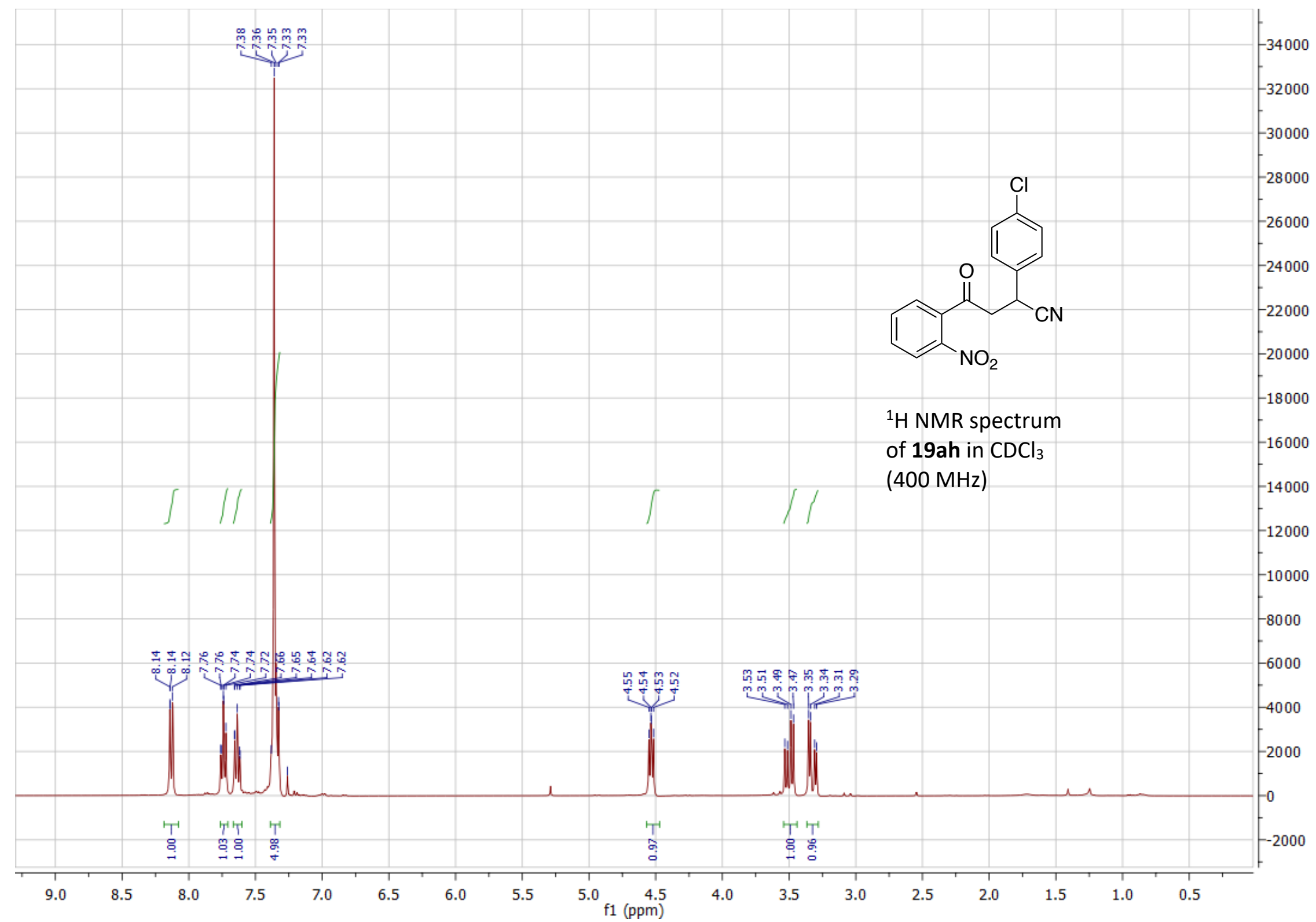




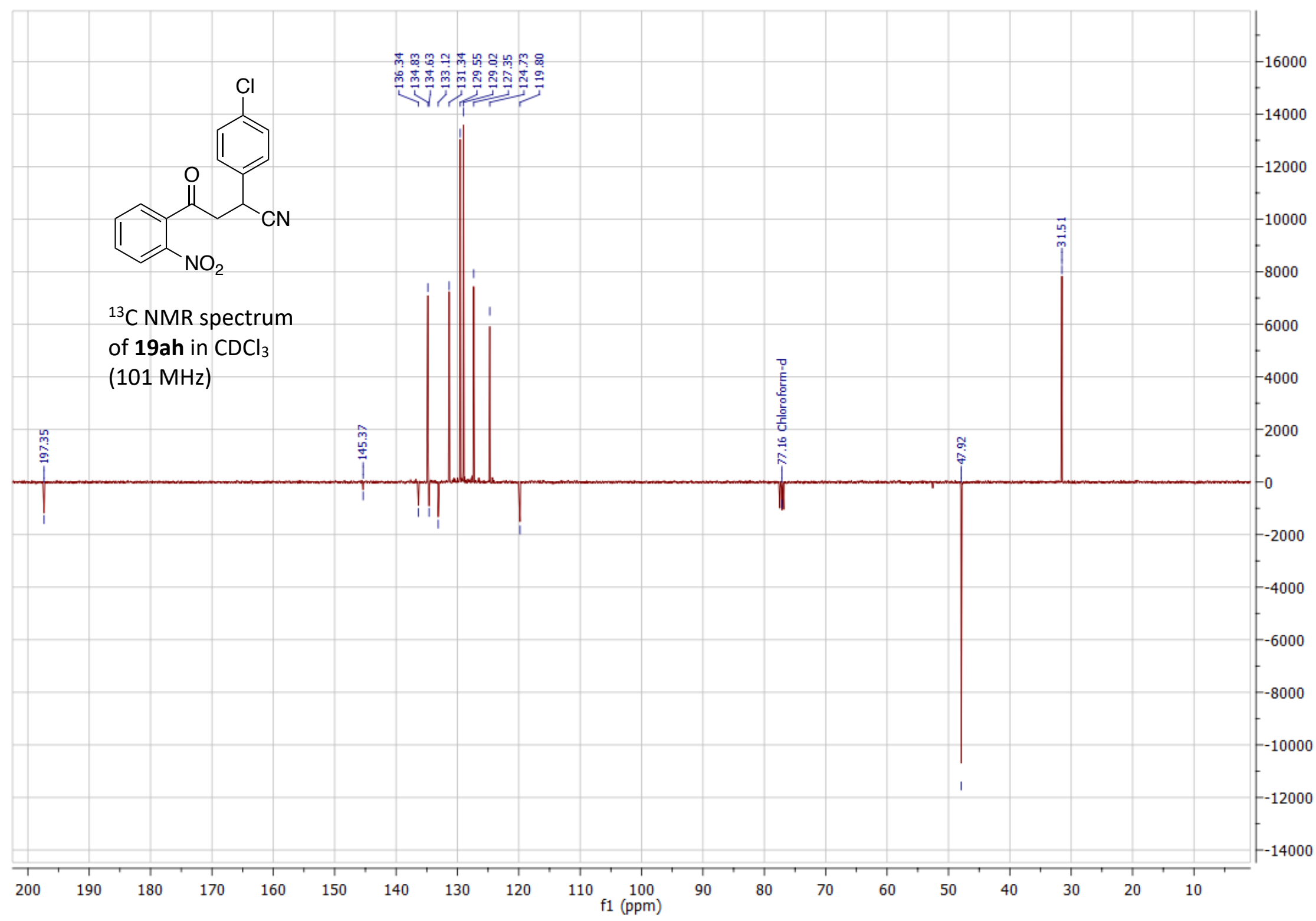




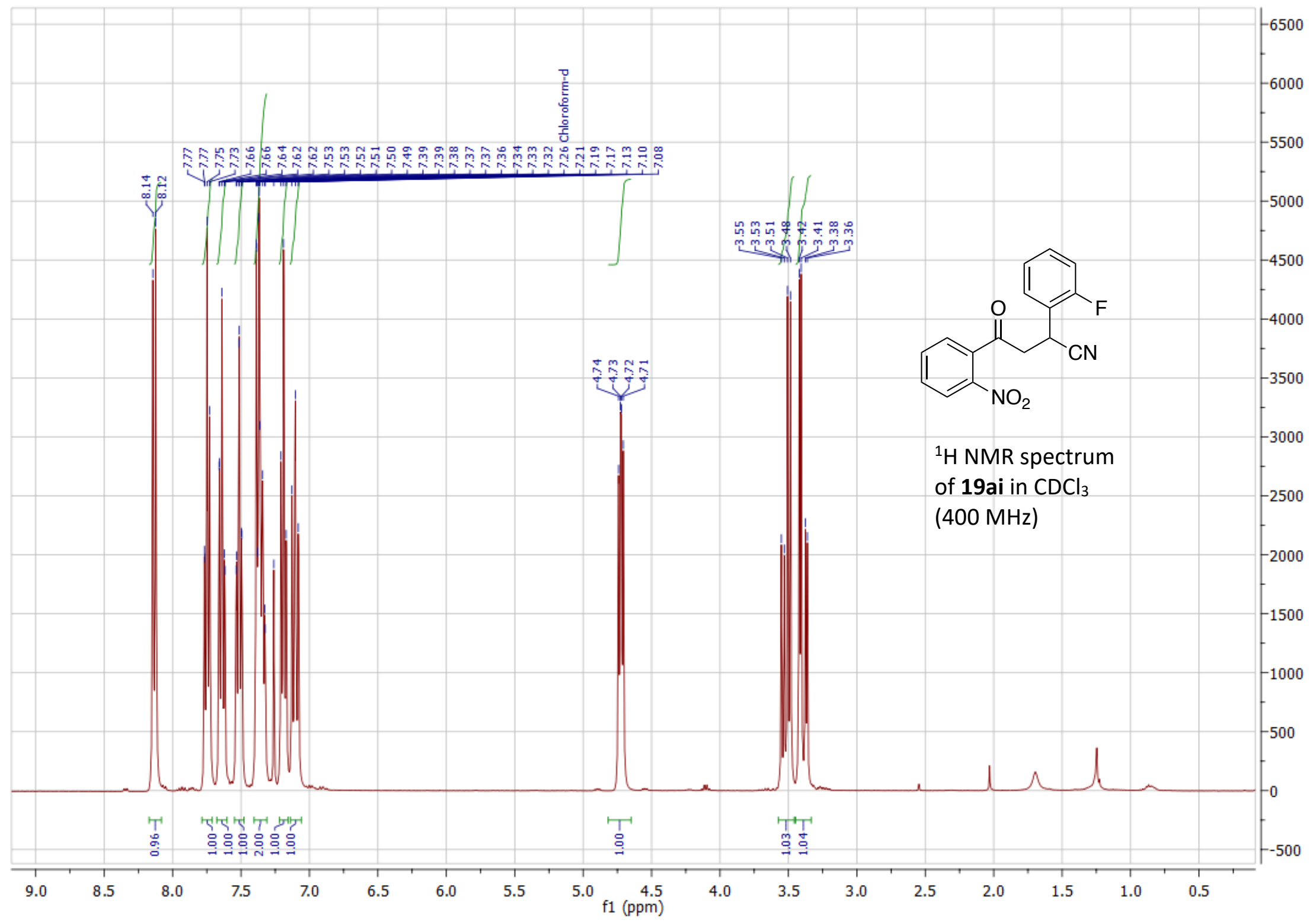




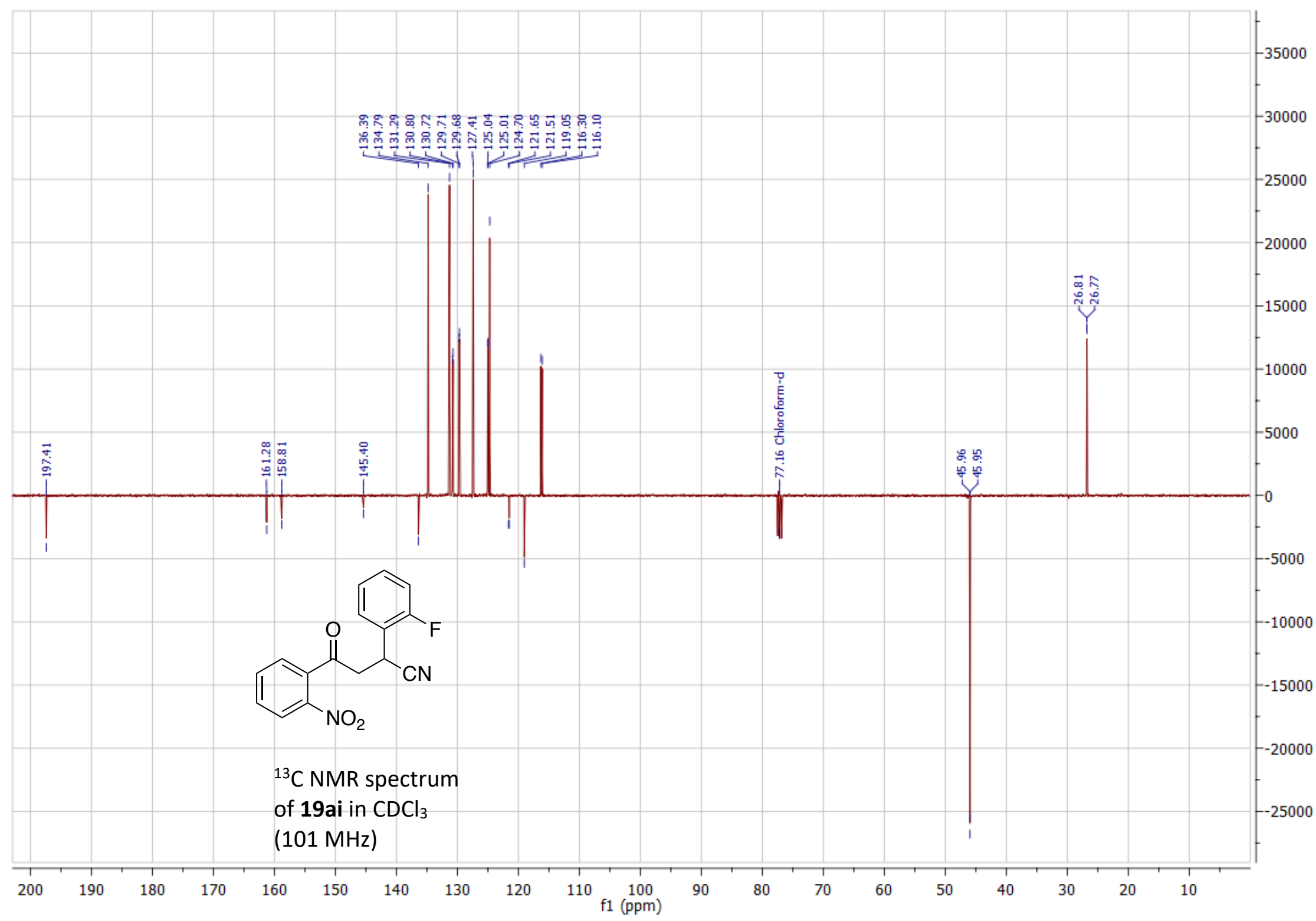




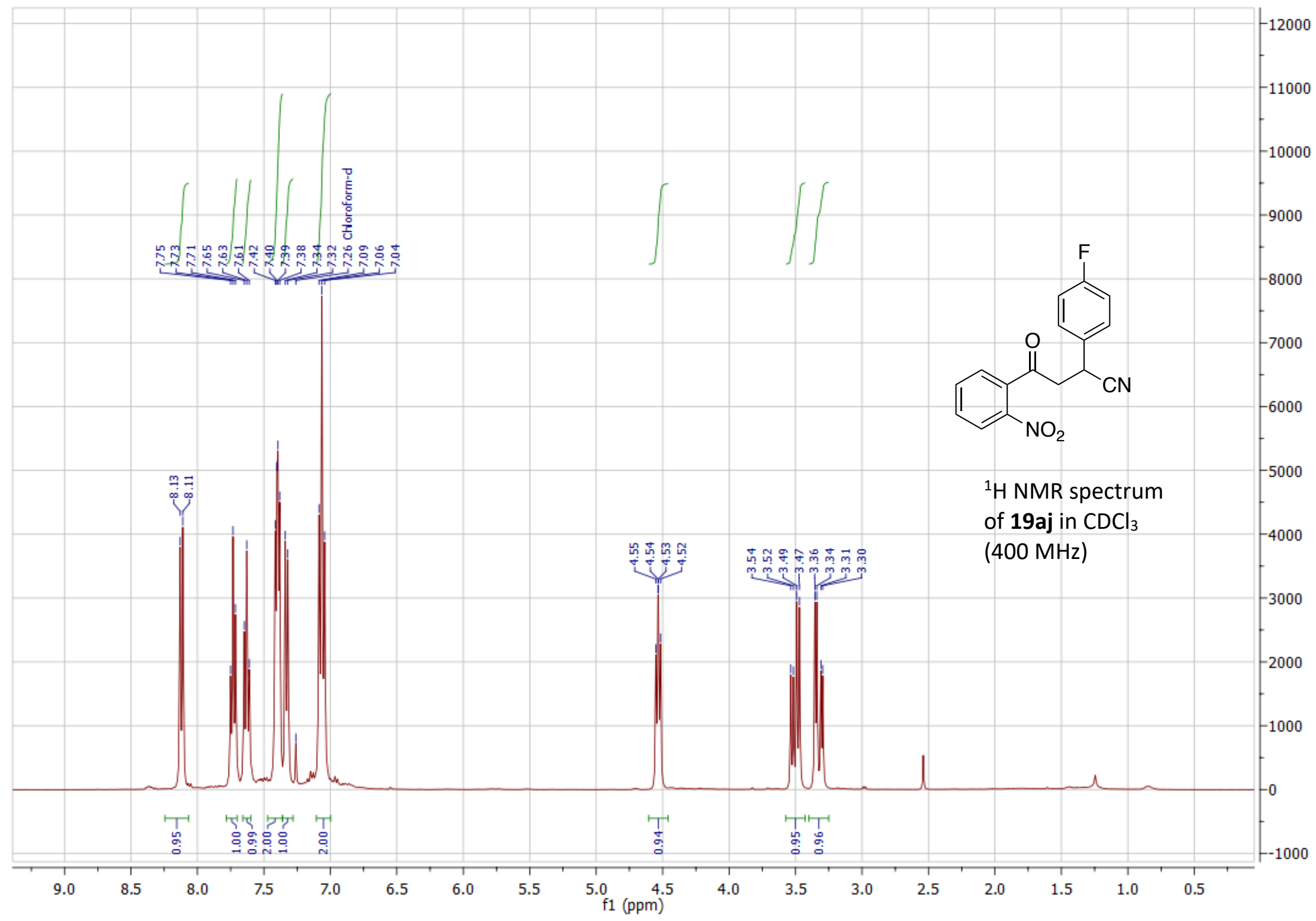




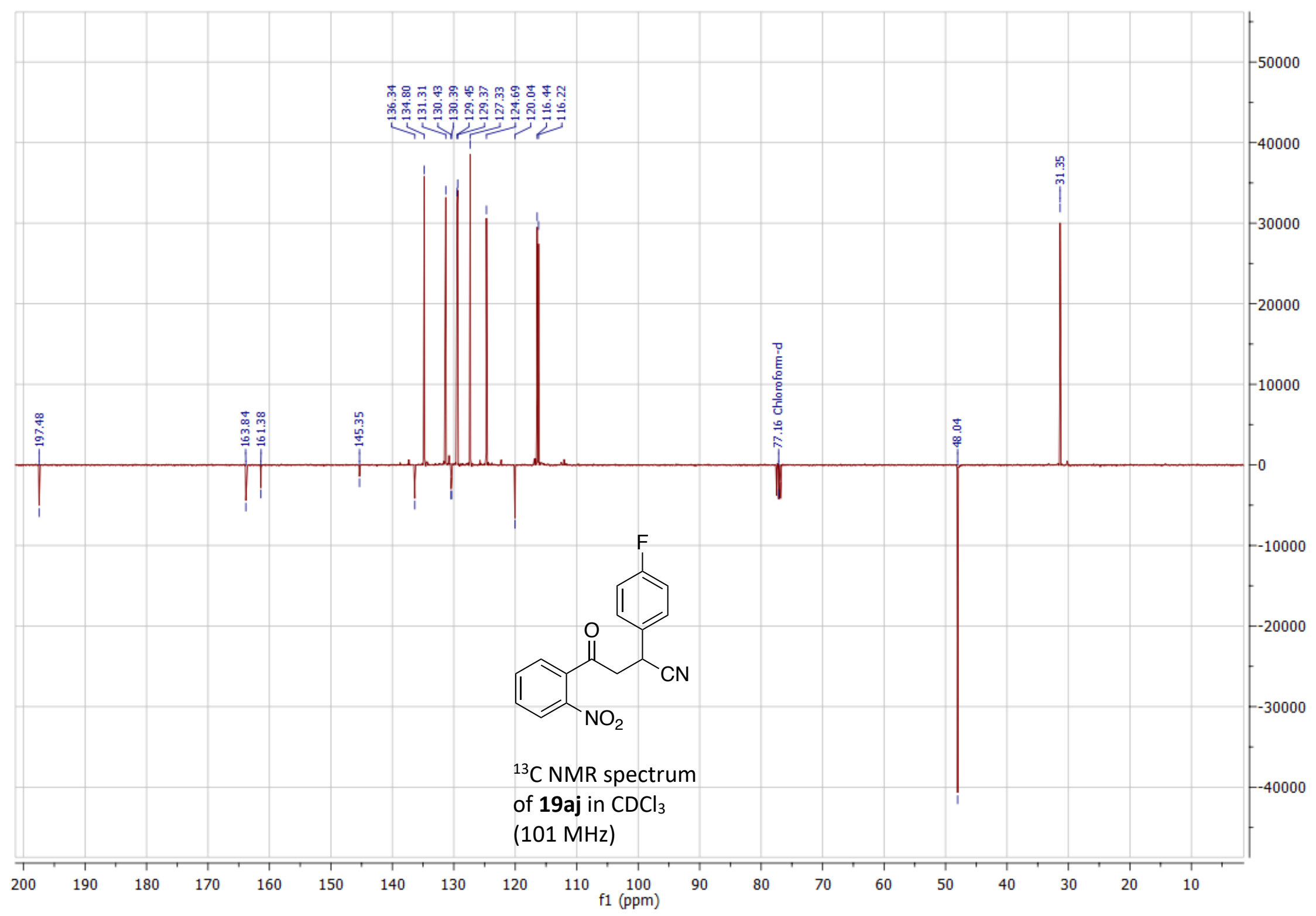




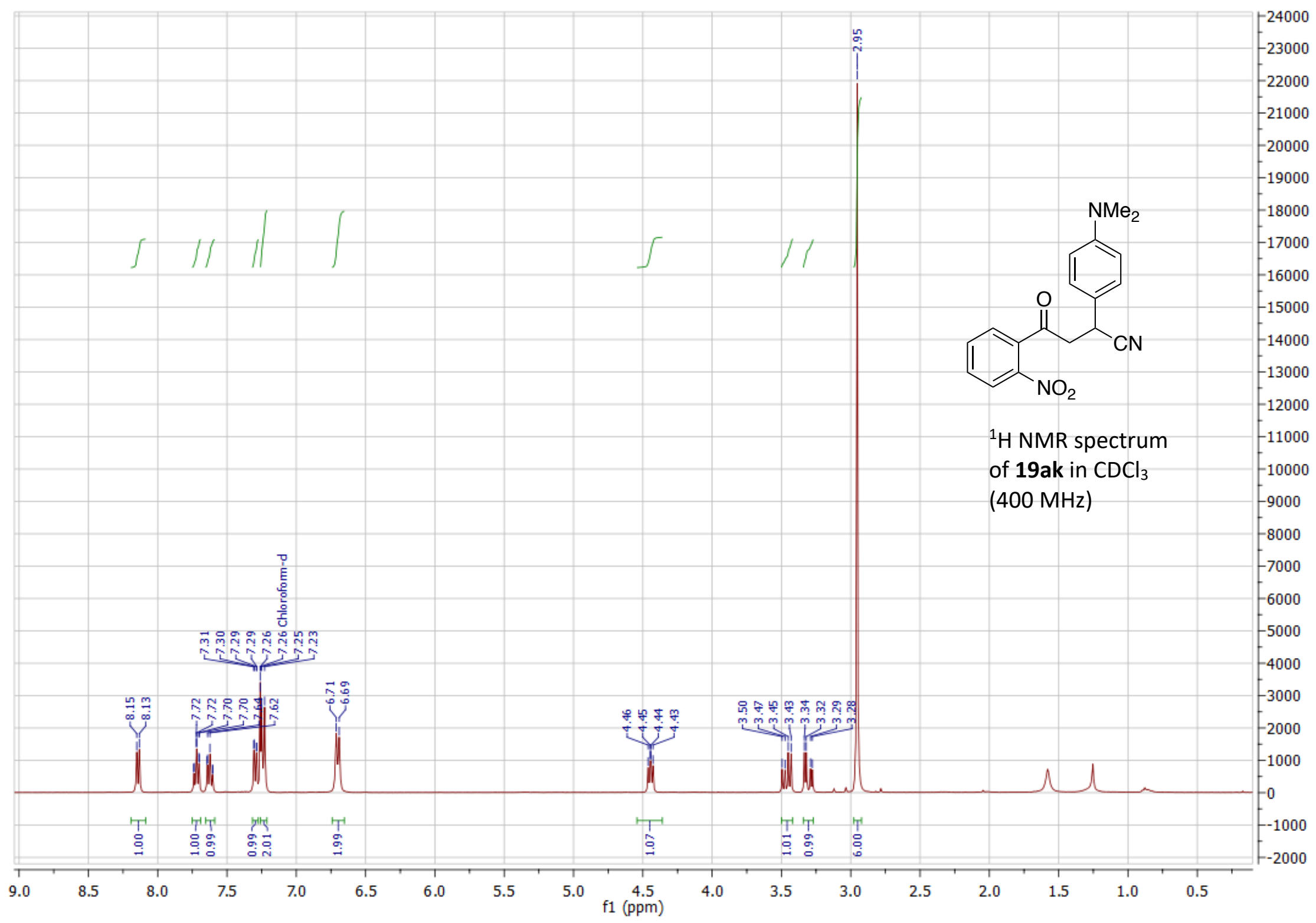




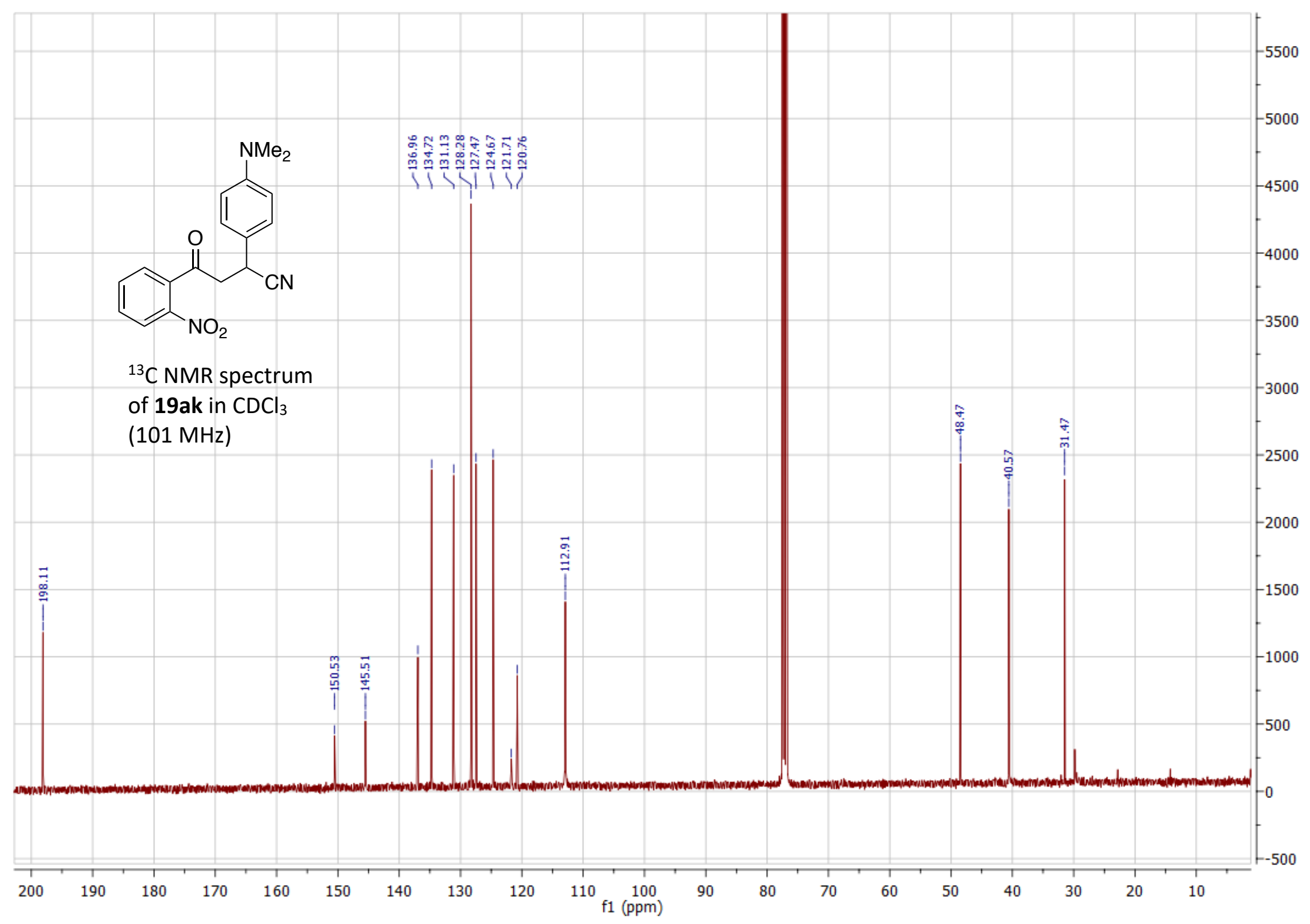




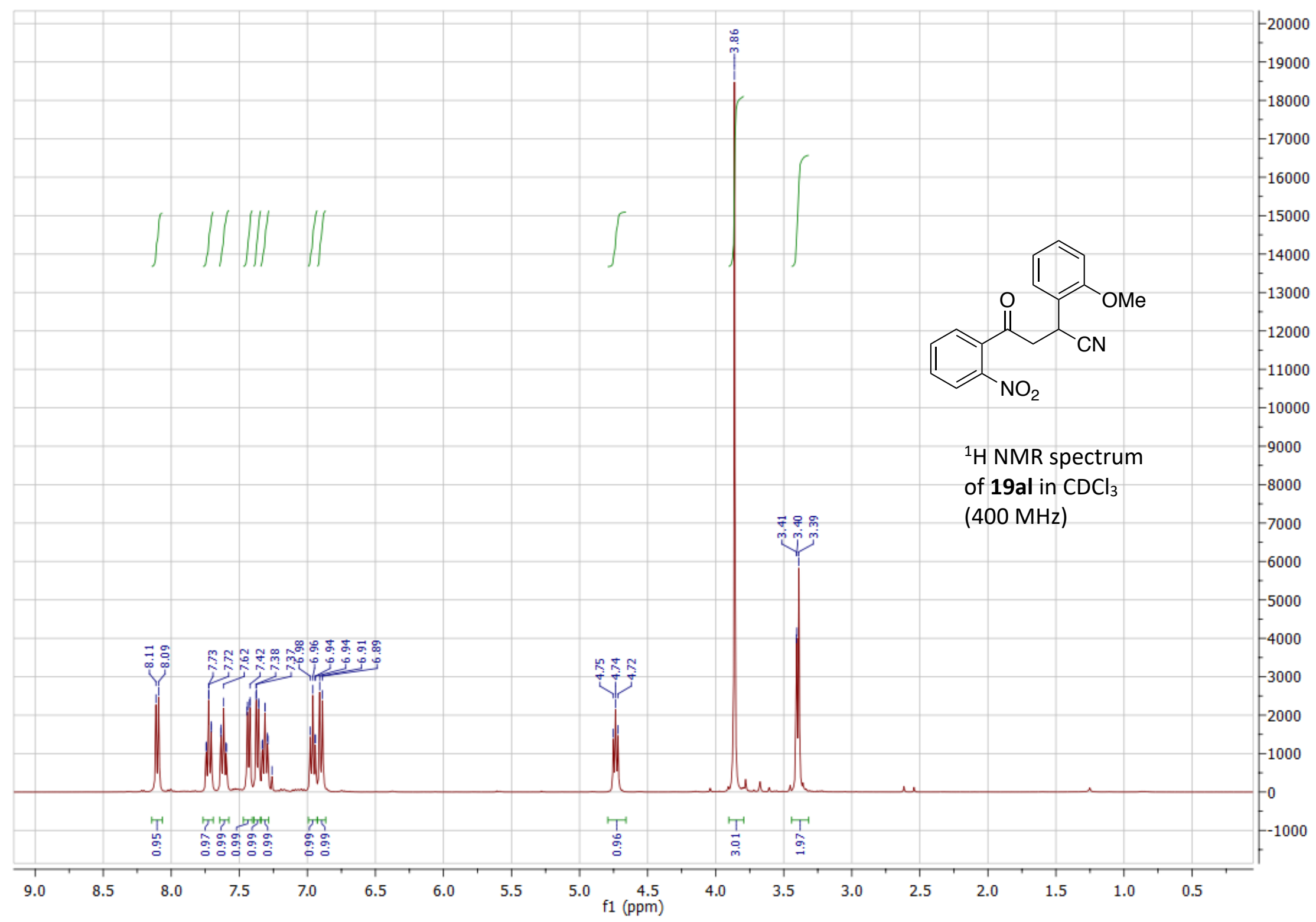




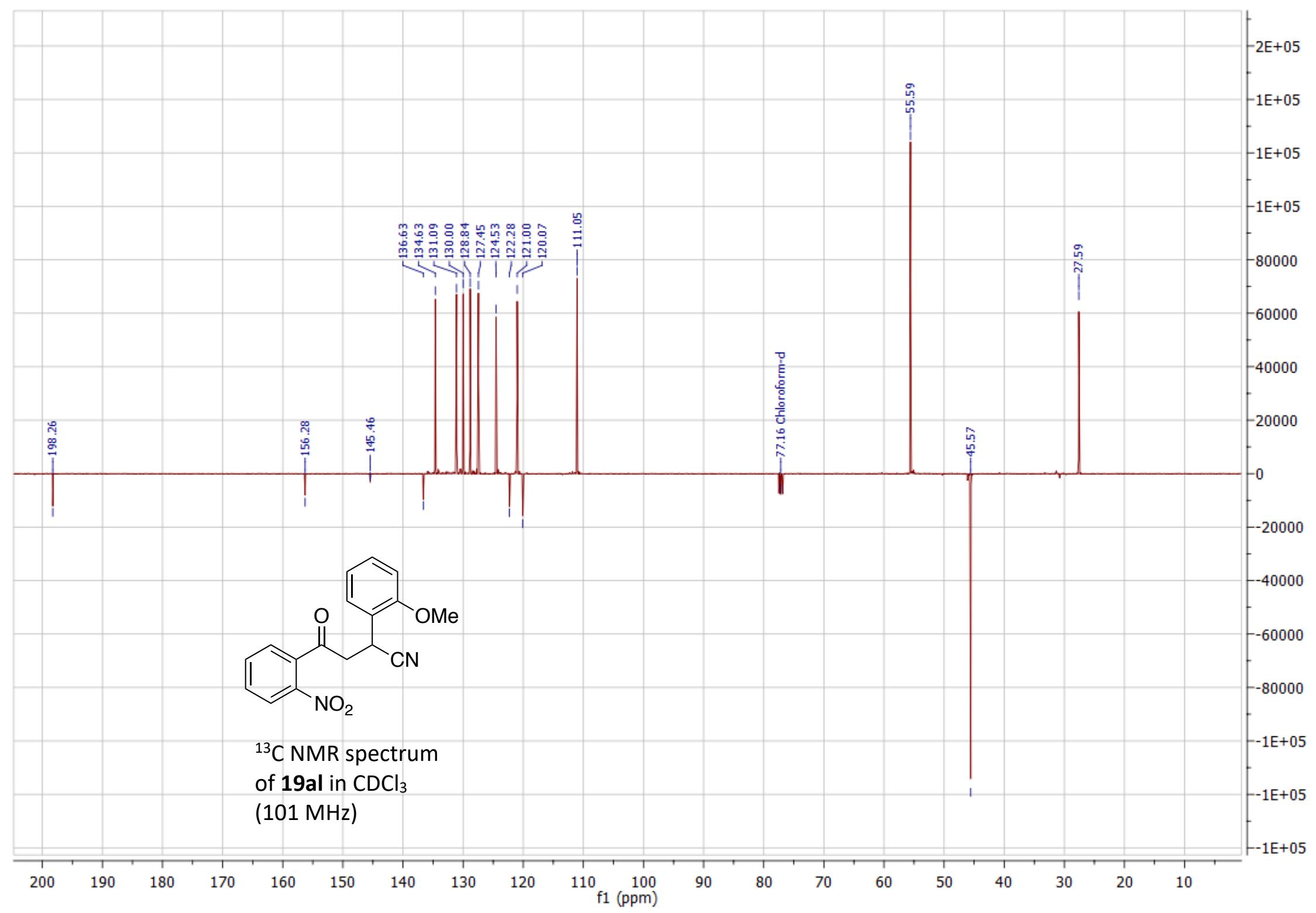




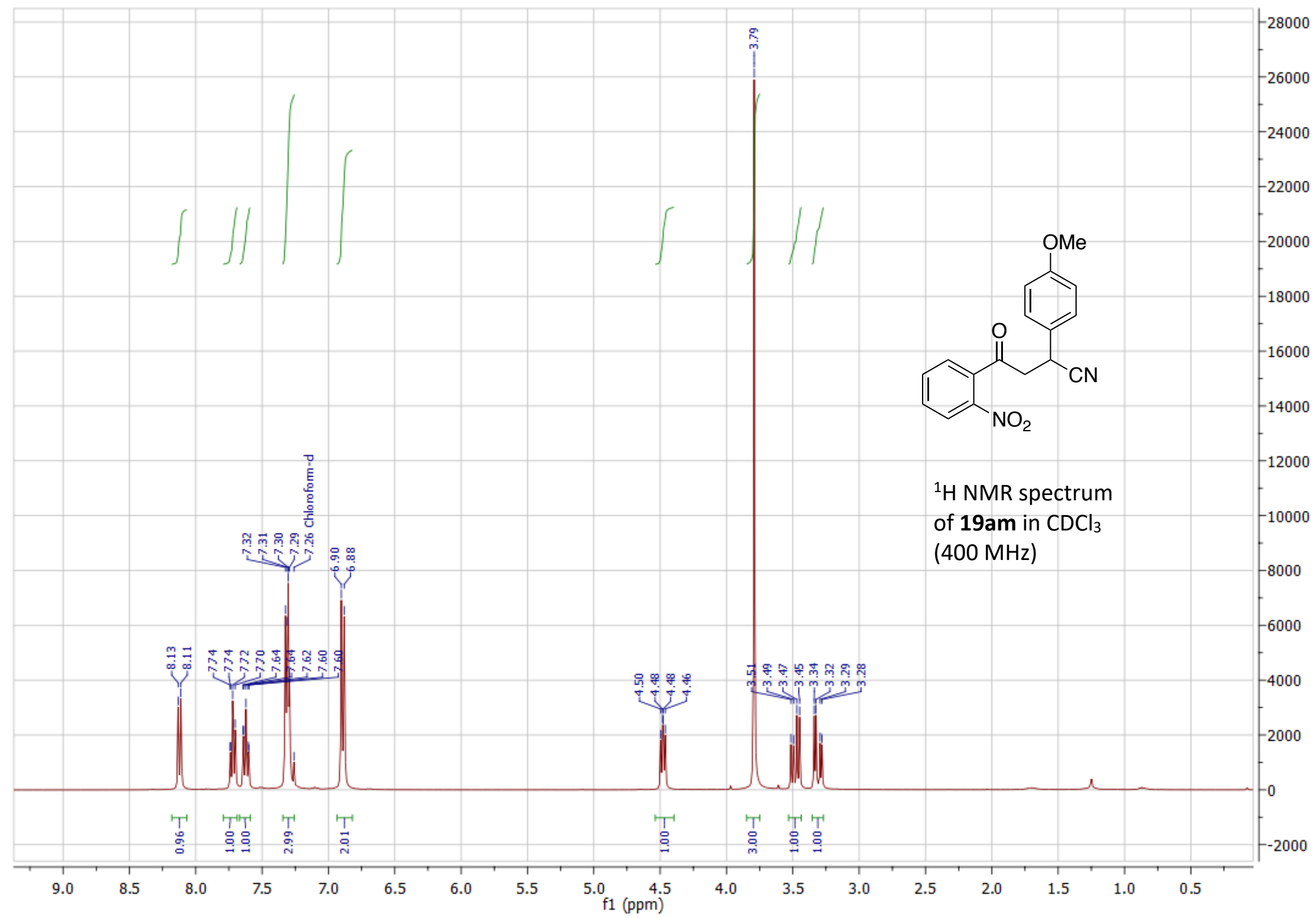




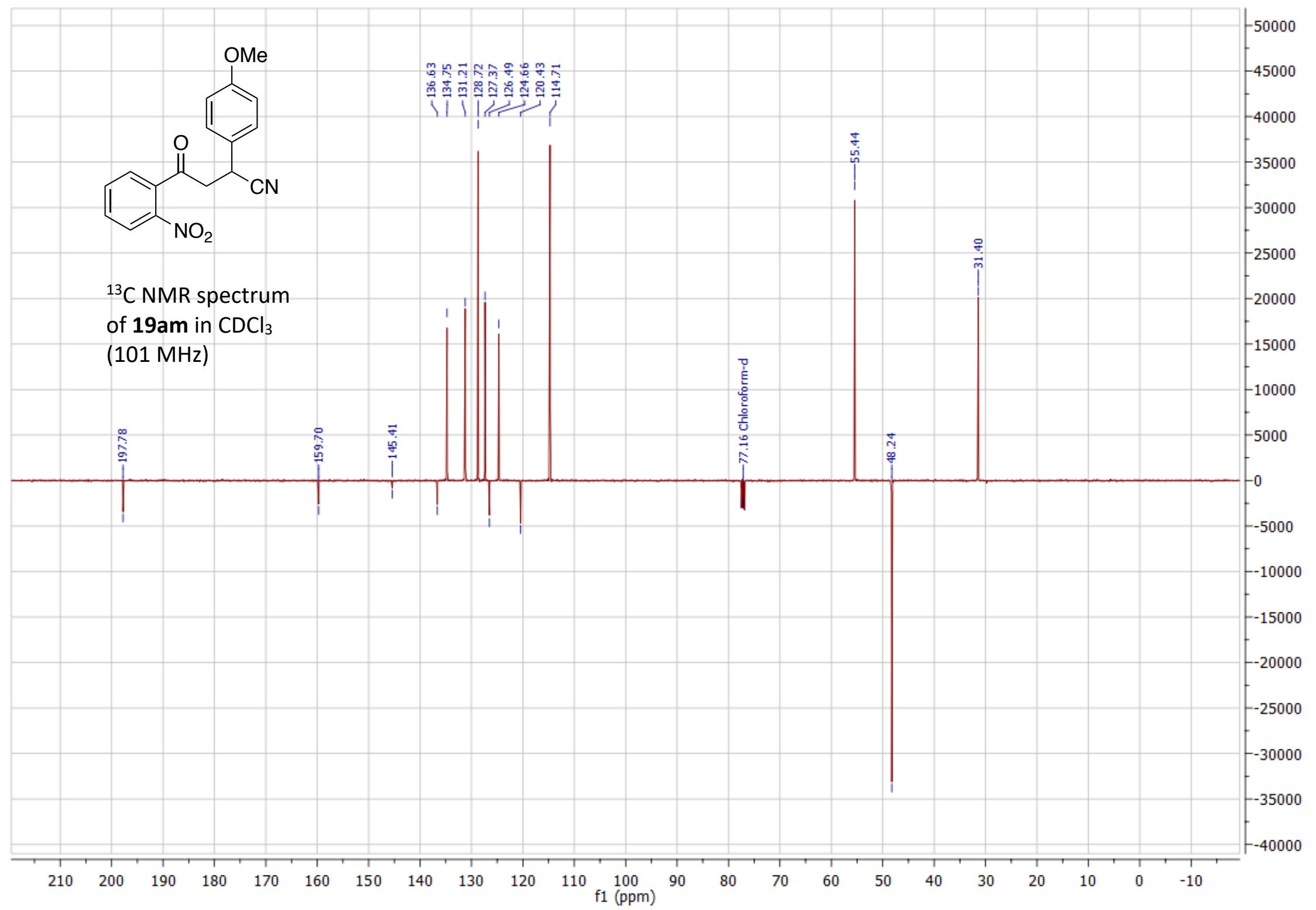




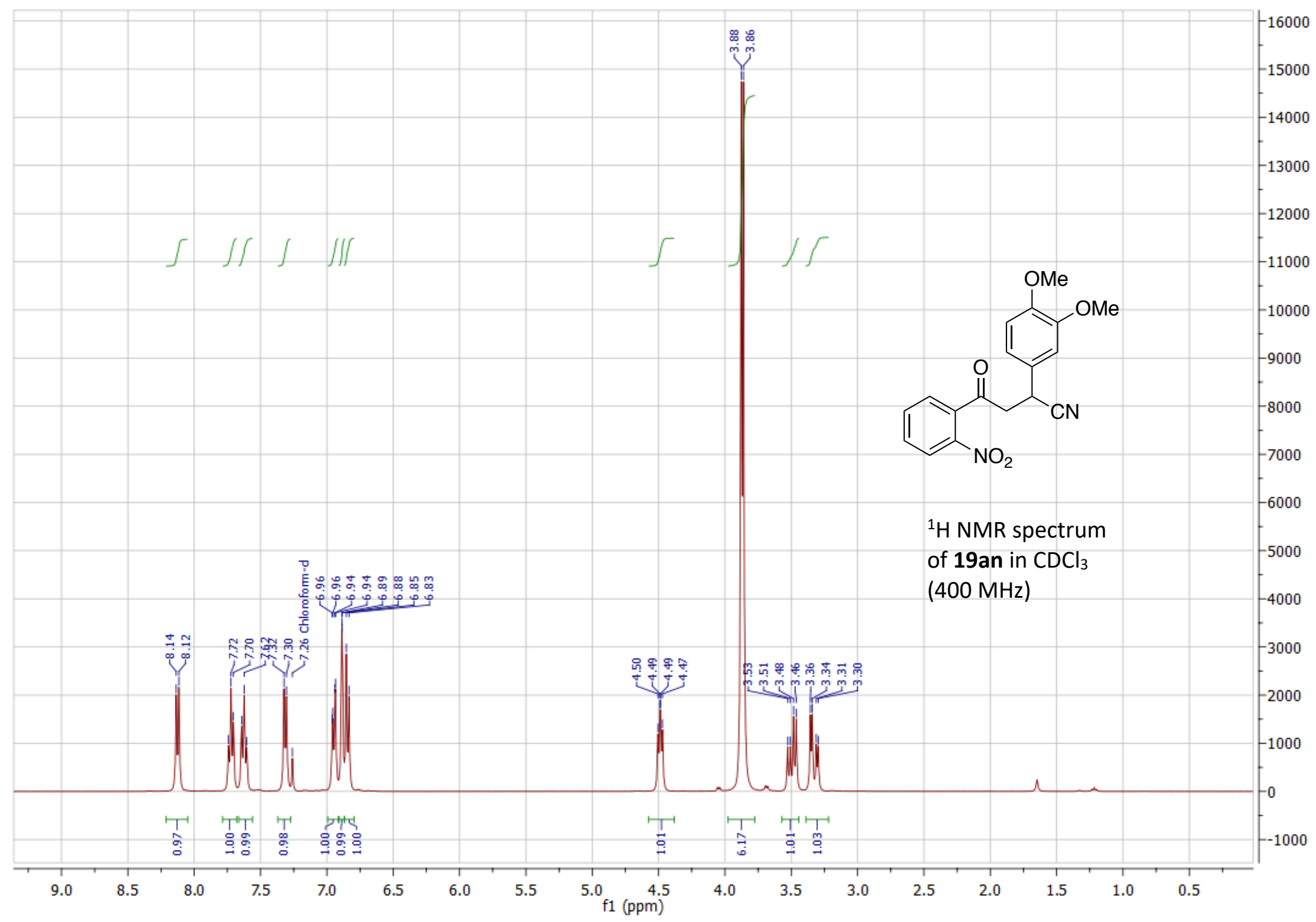




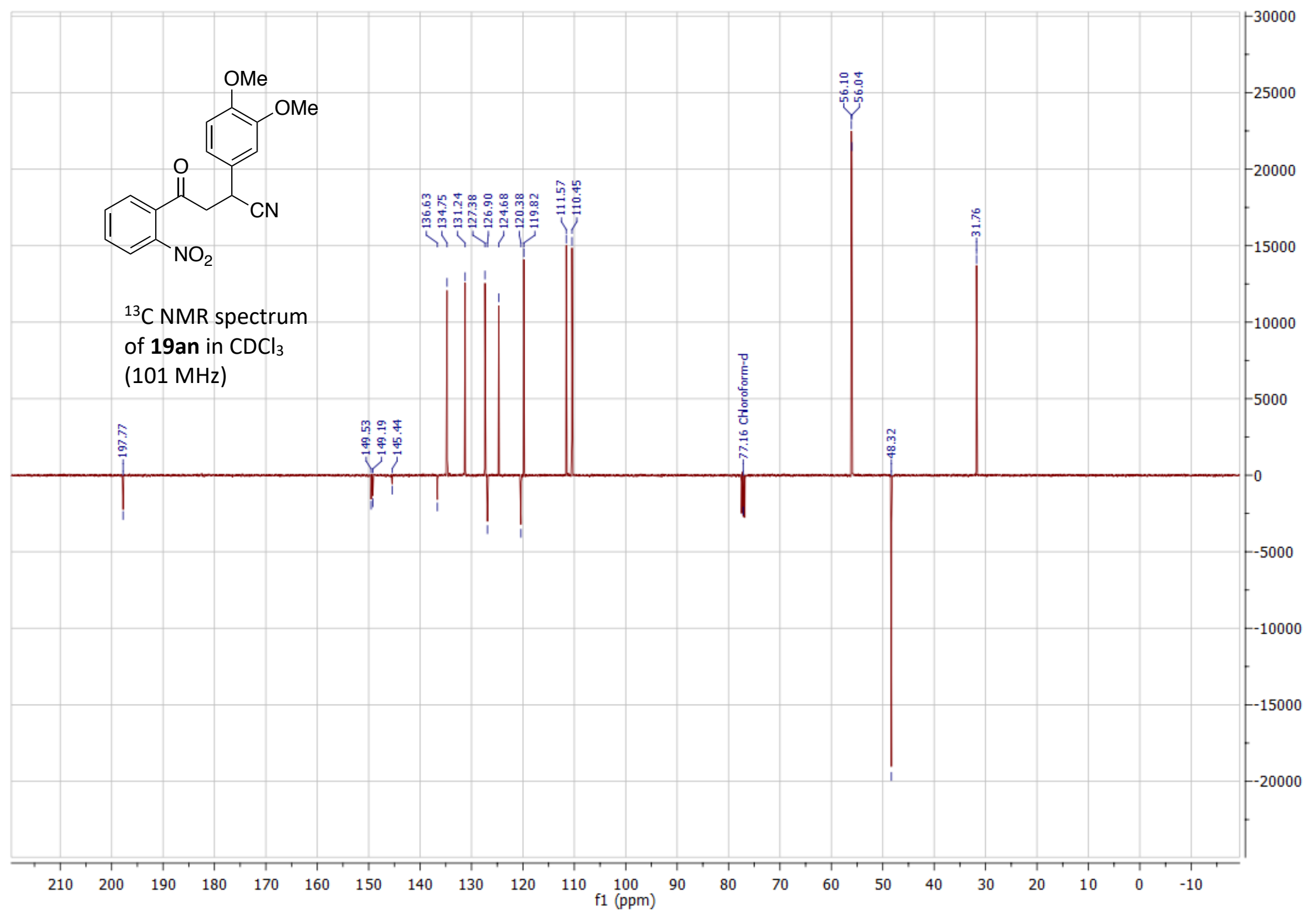




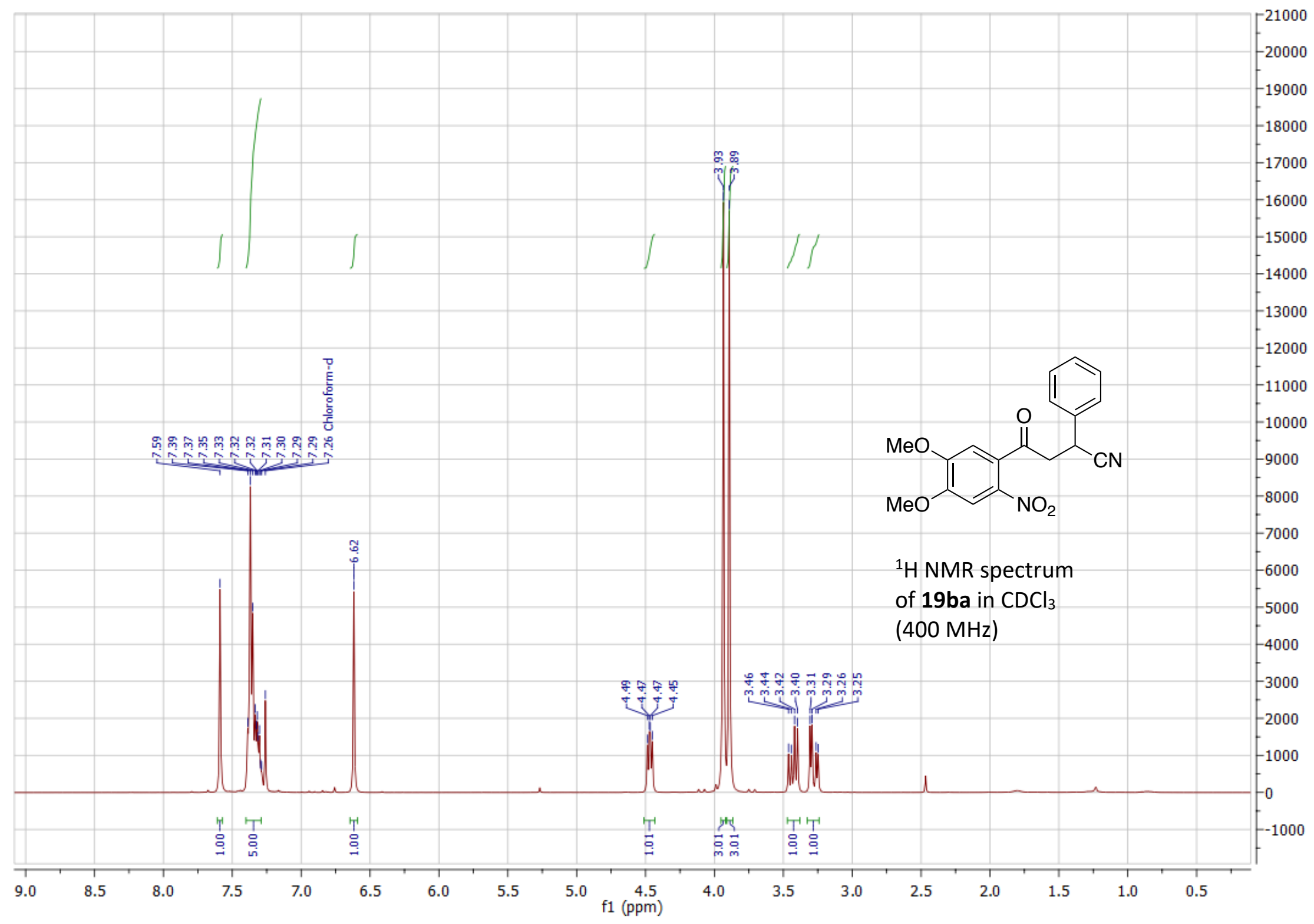




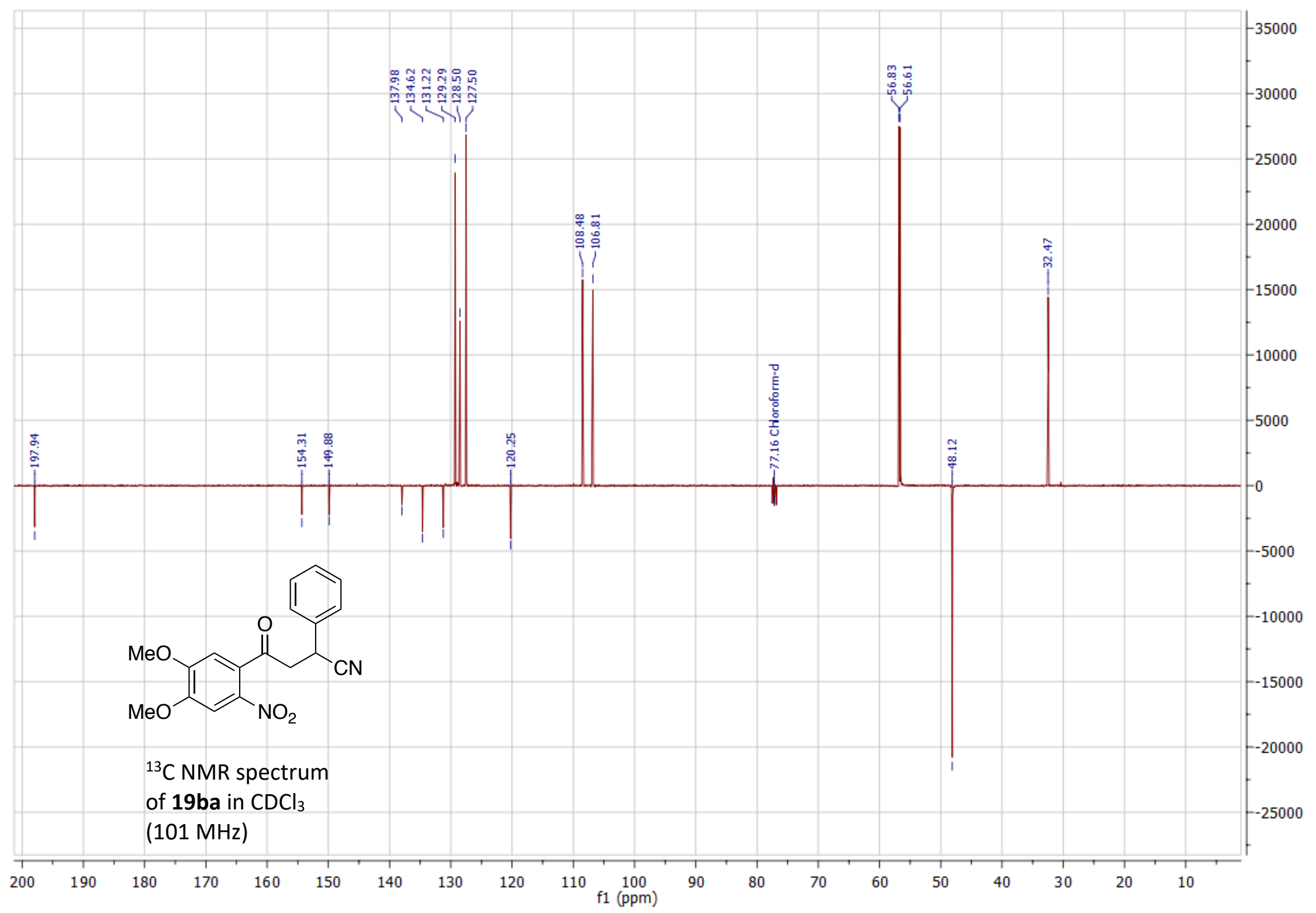




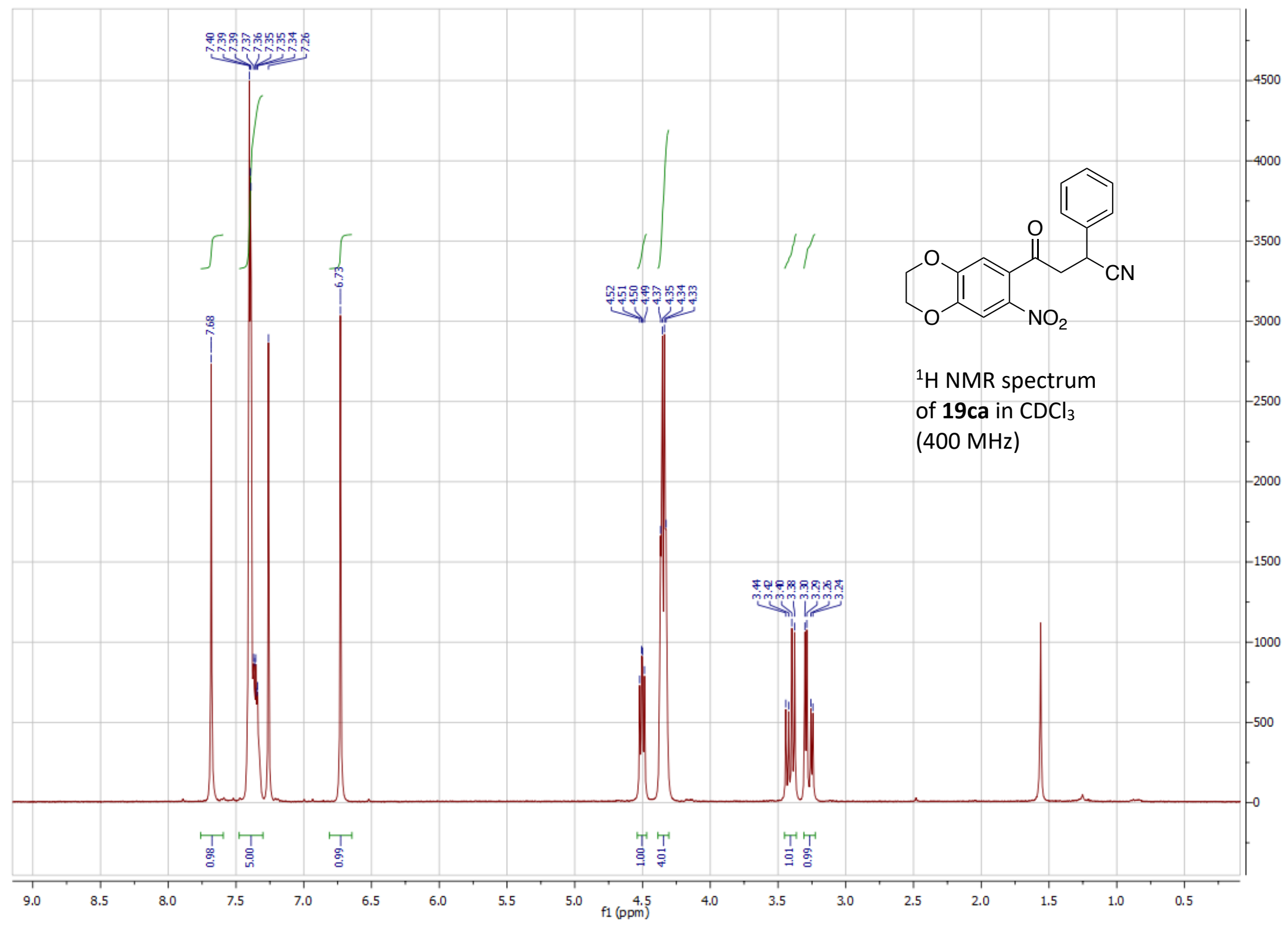




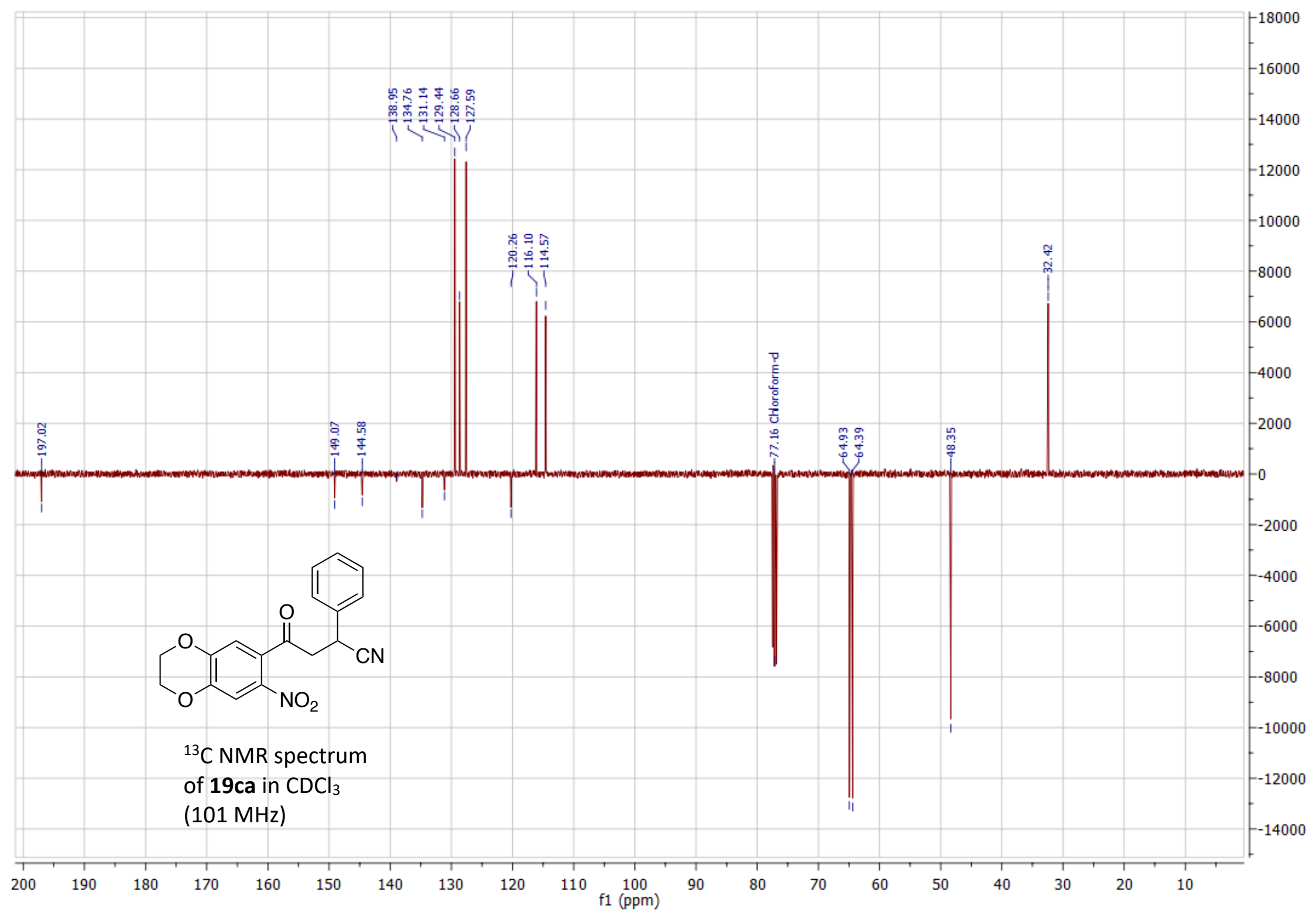


${ }^{1} \mathrm{H}$ and ${ }^{13} \mathrm{C}$ Spectral Charts of (E)-1-(2-aminophenyl)-3-arylprop-2-en-1-ones 16

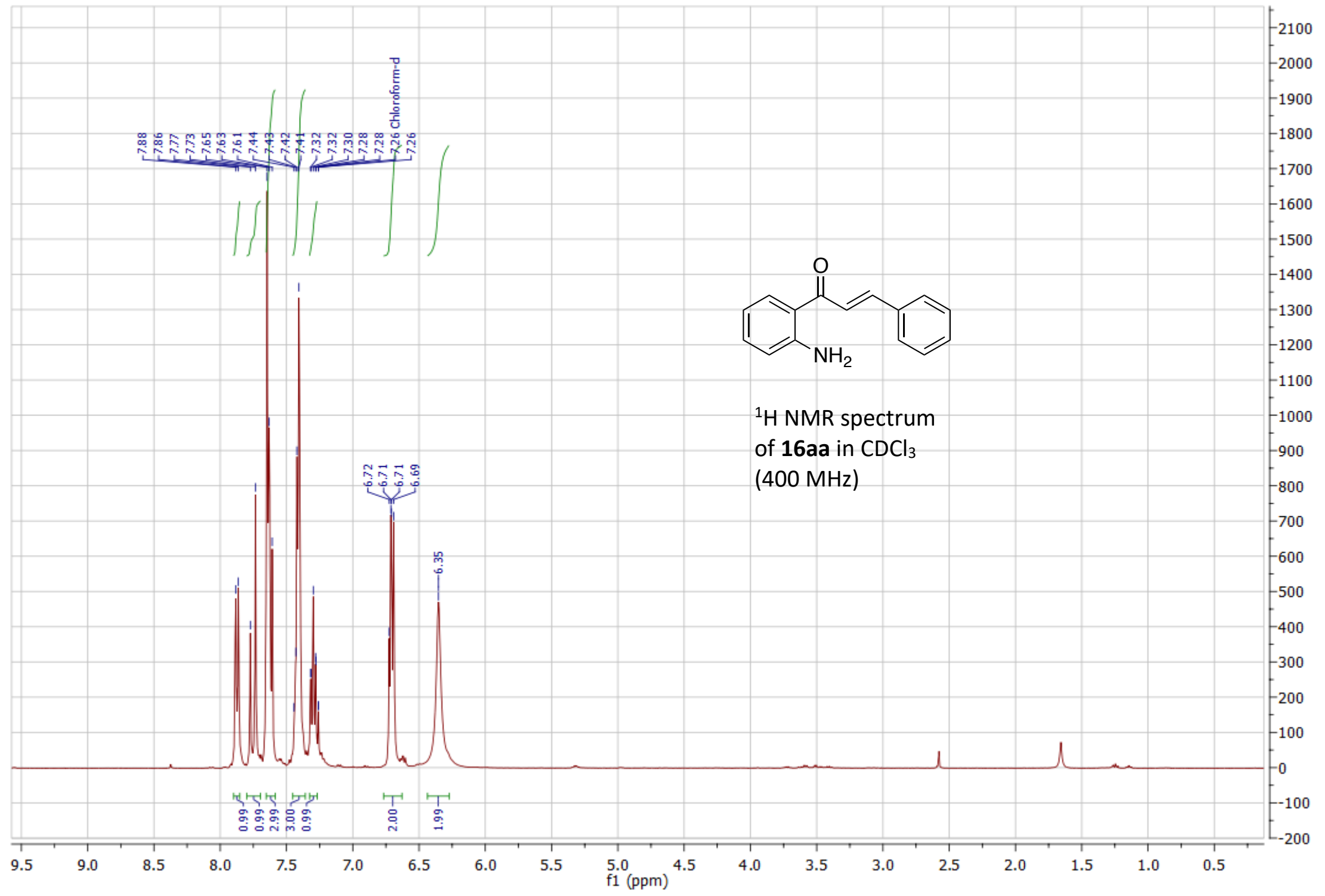




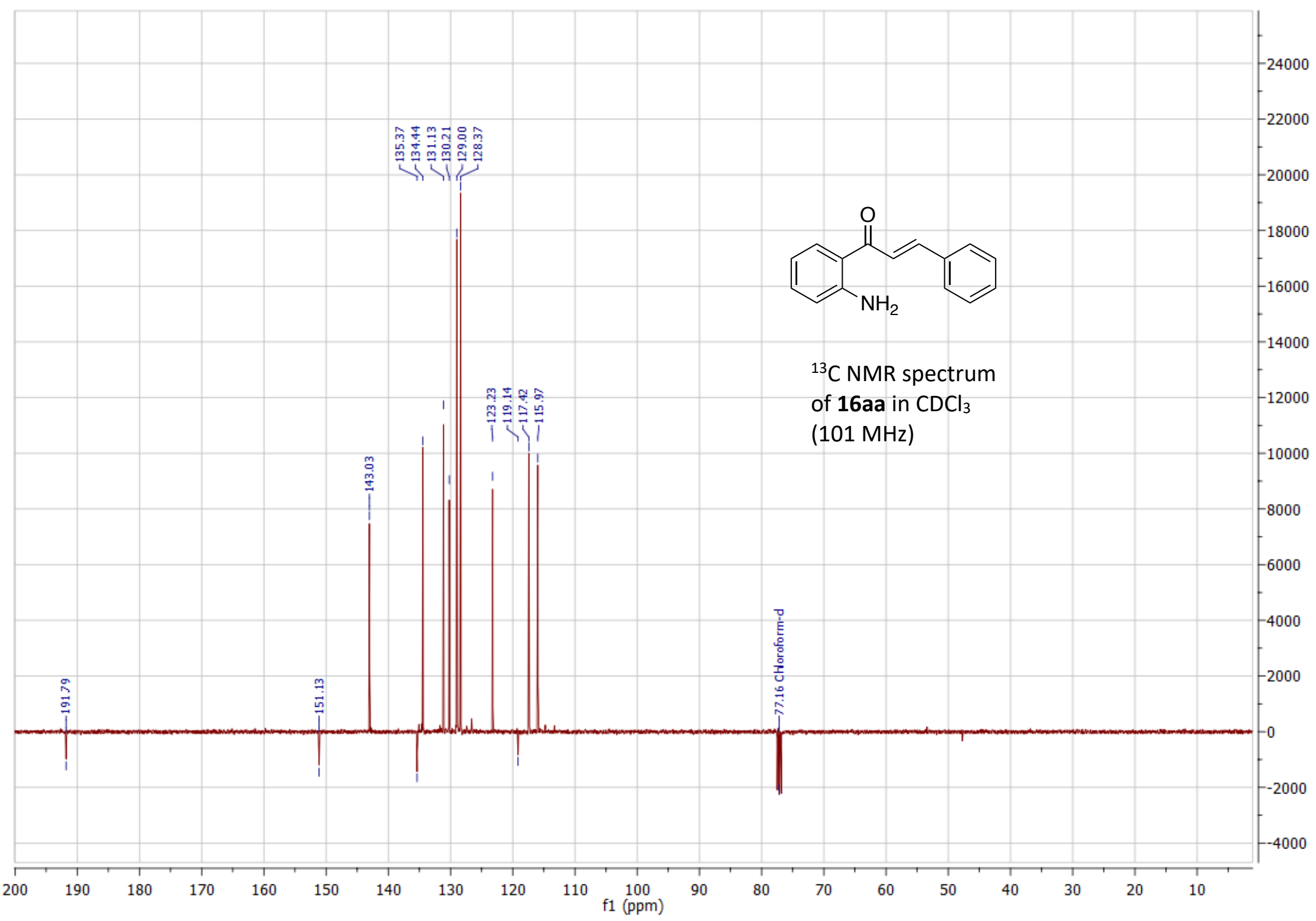




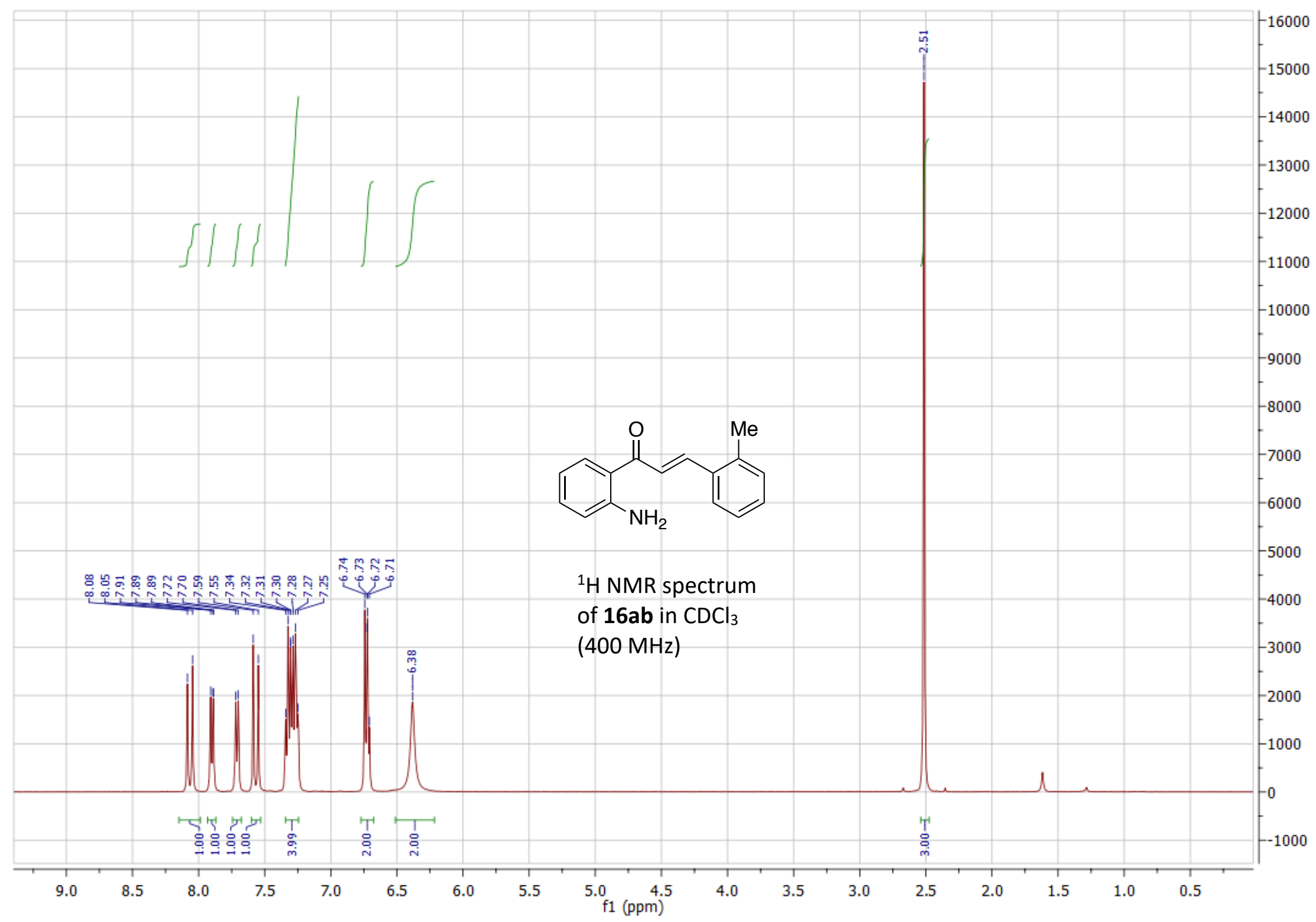




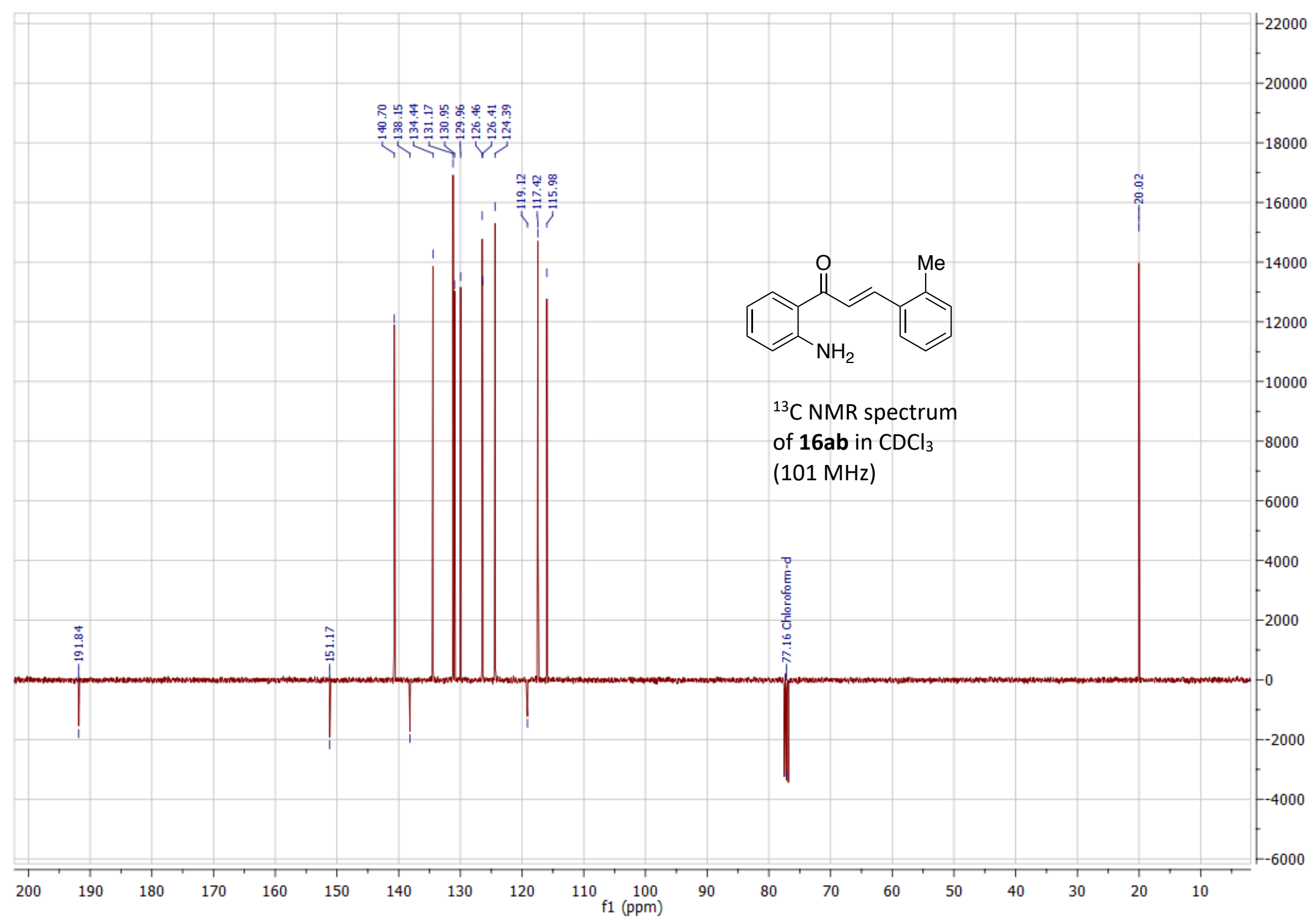




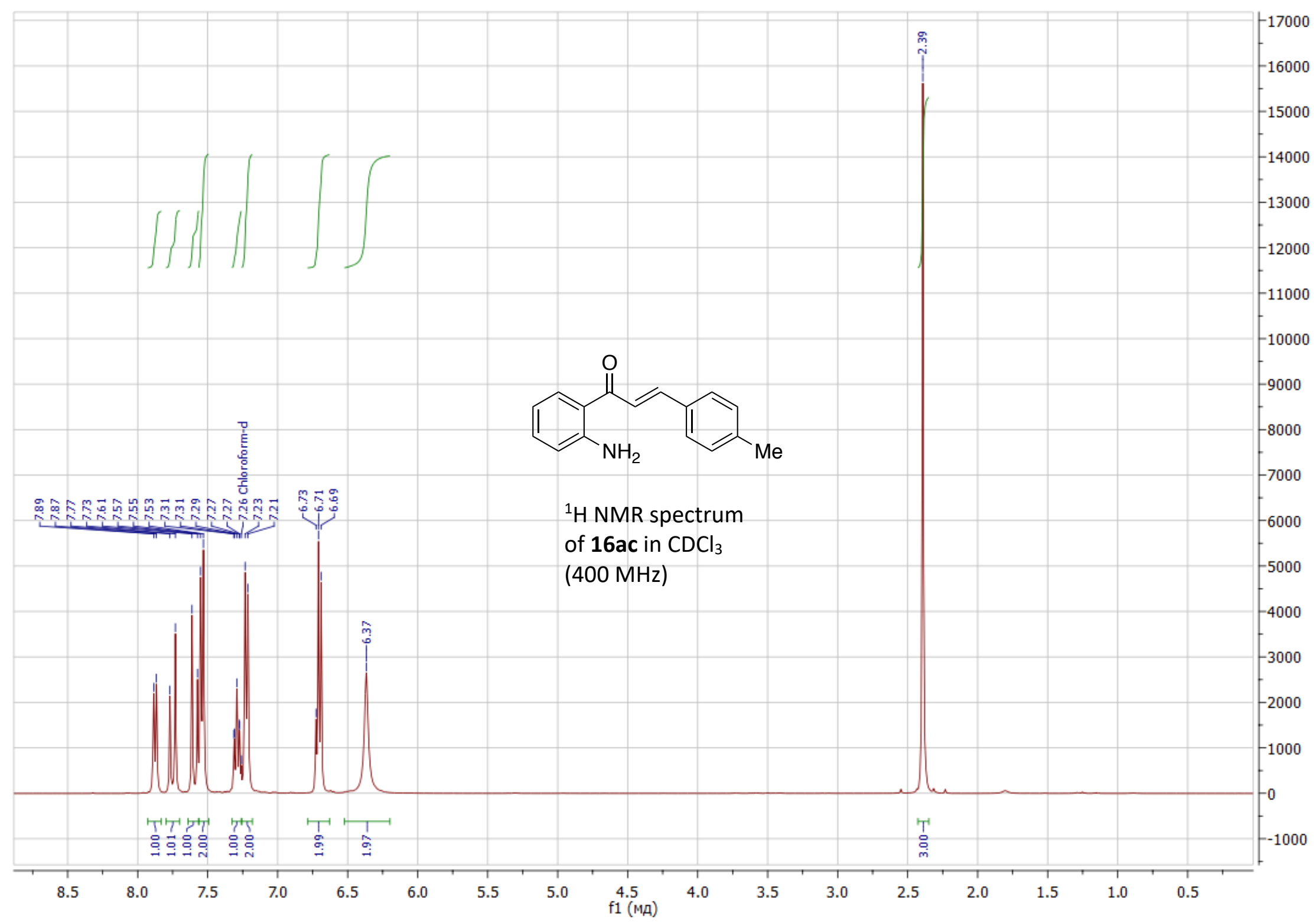




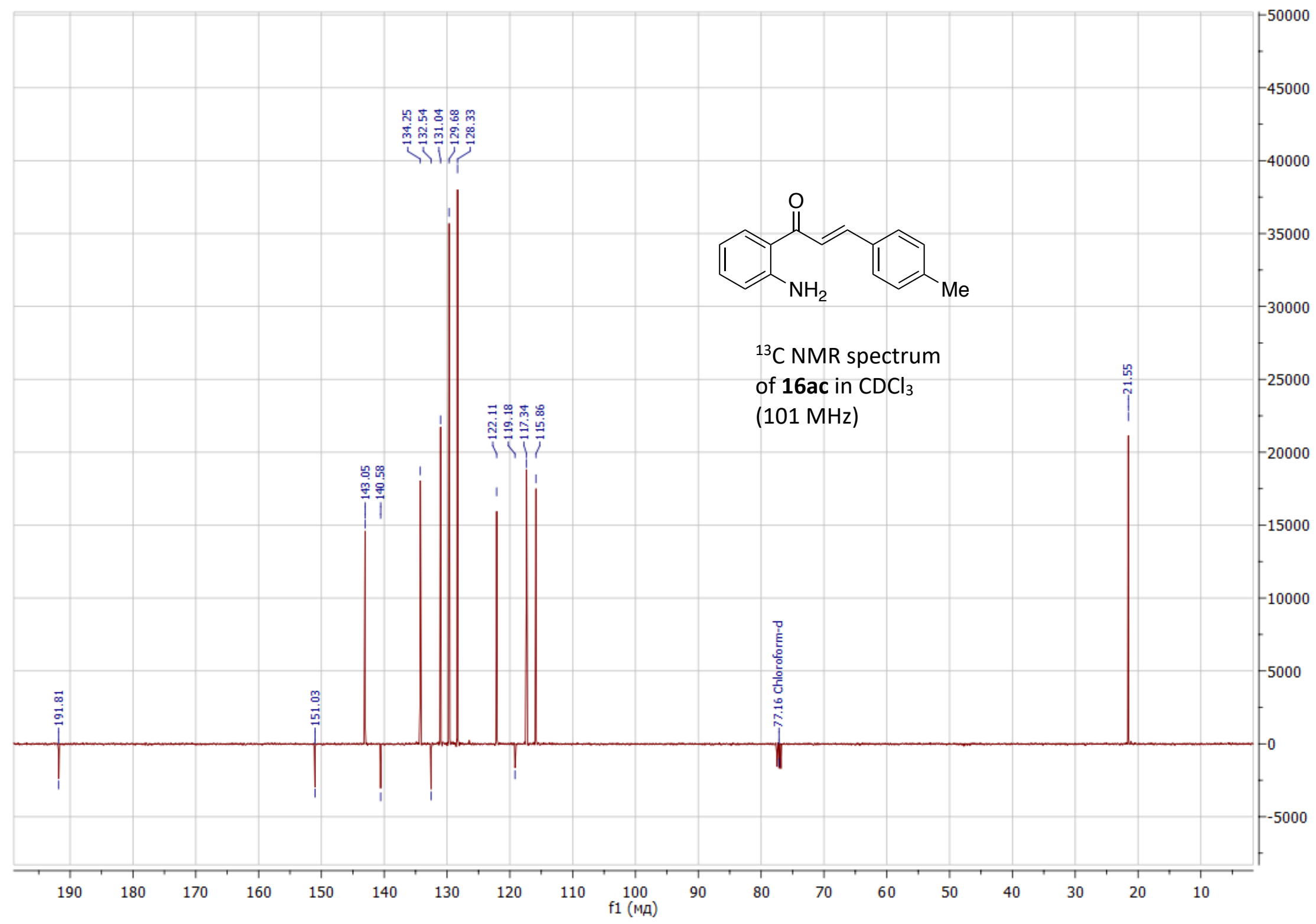




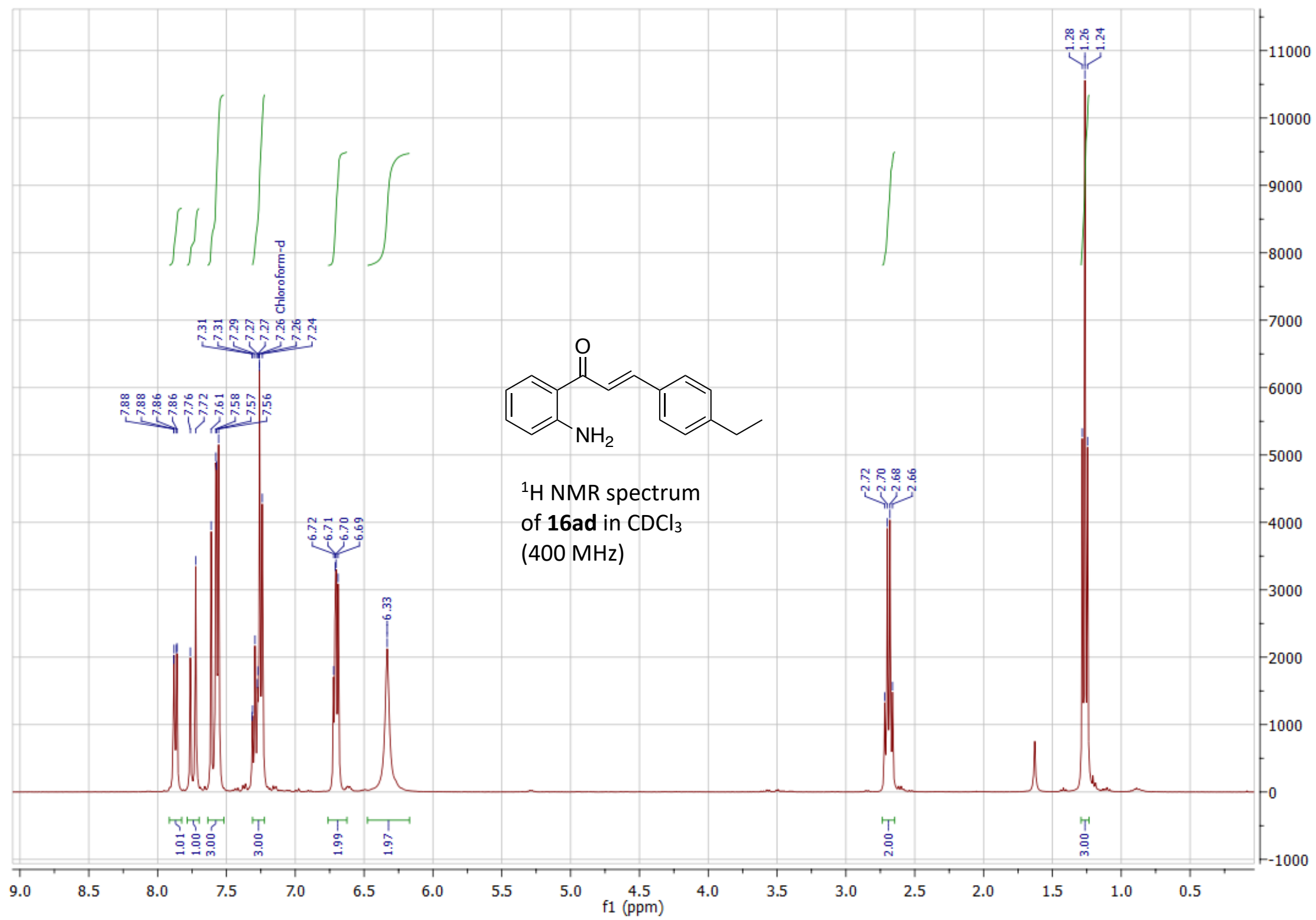




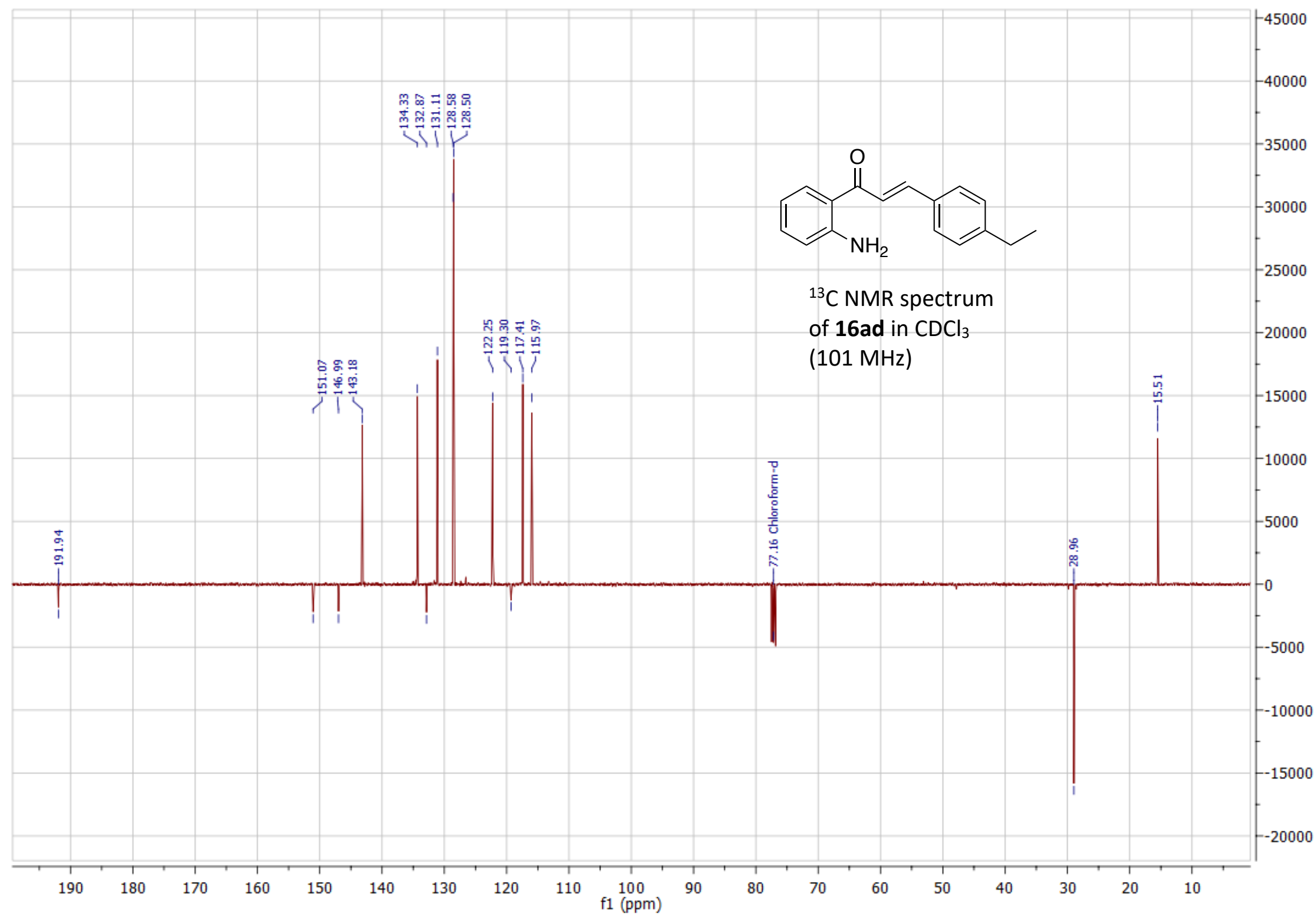




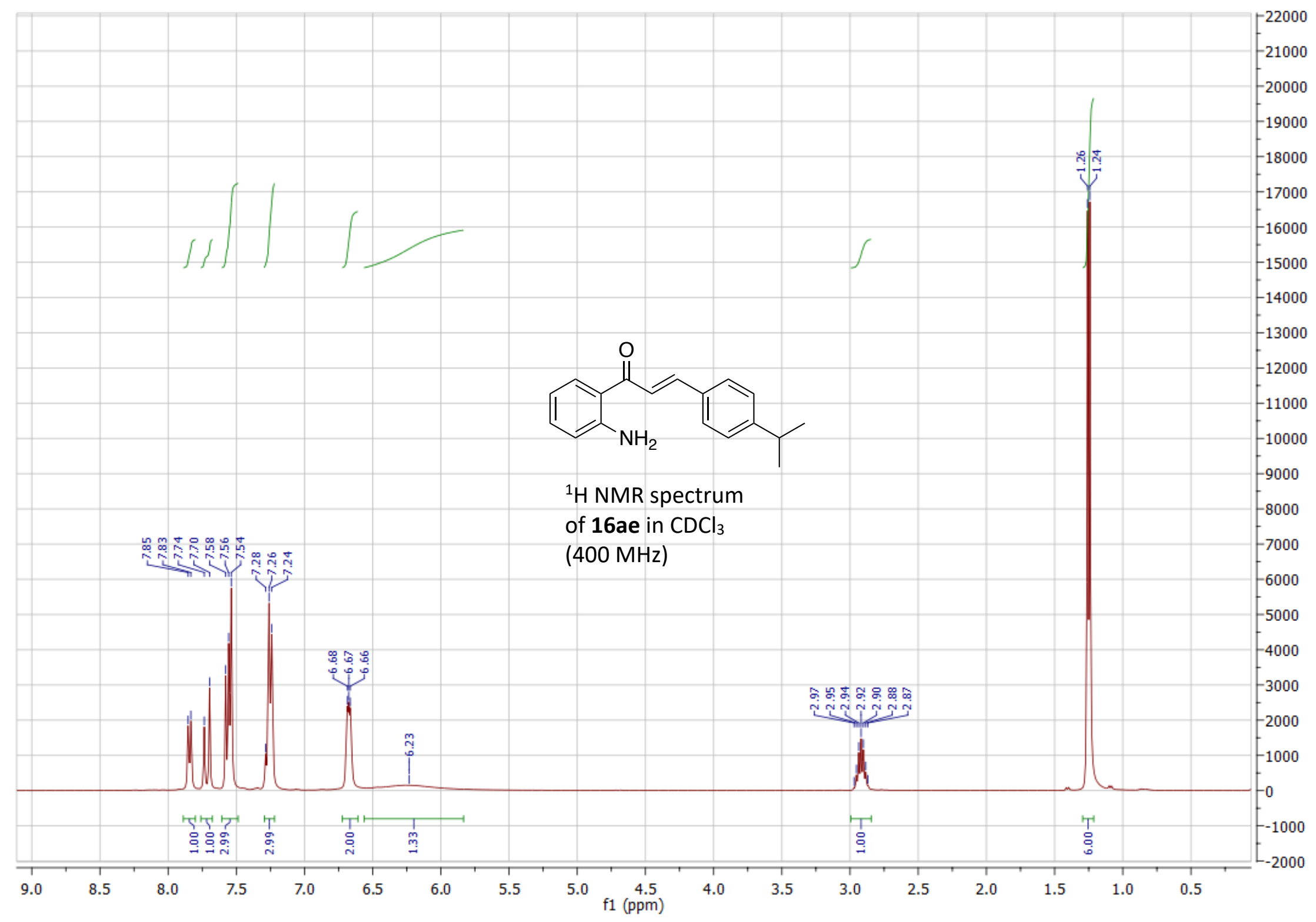




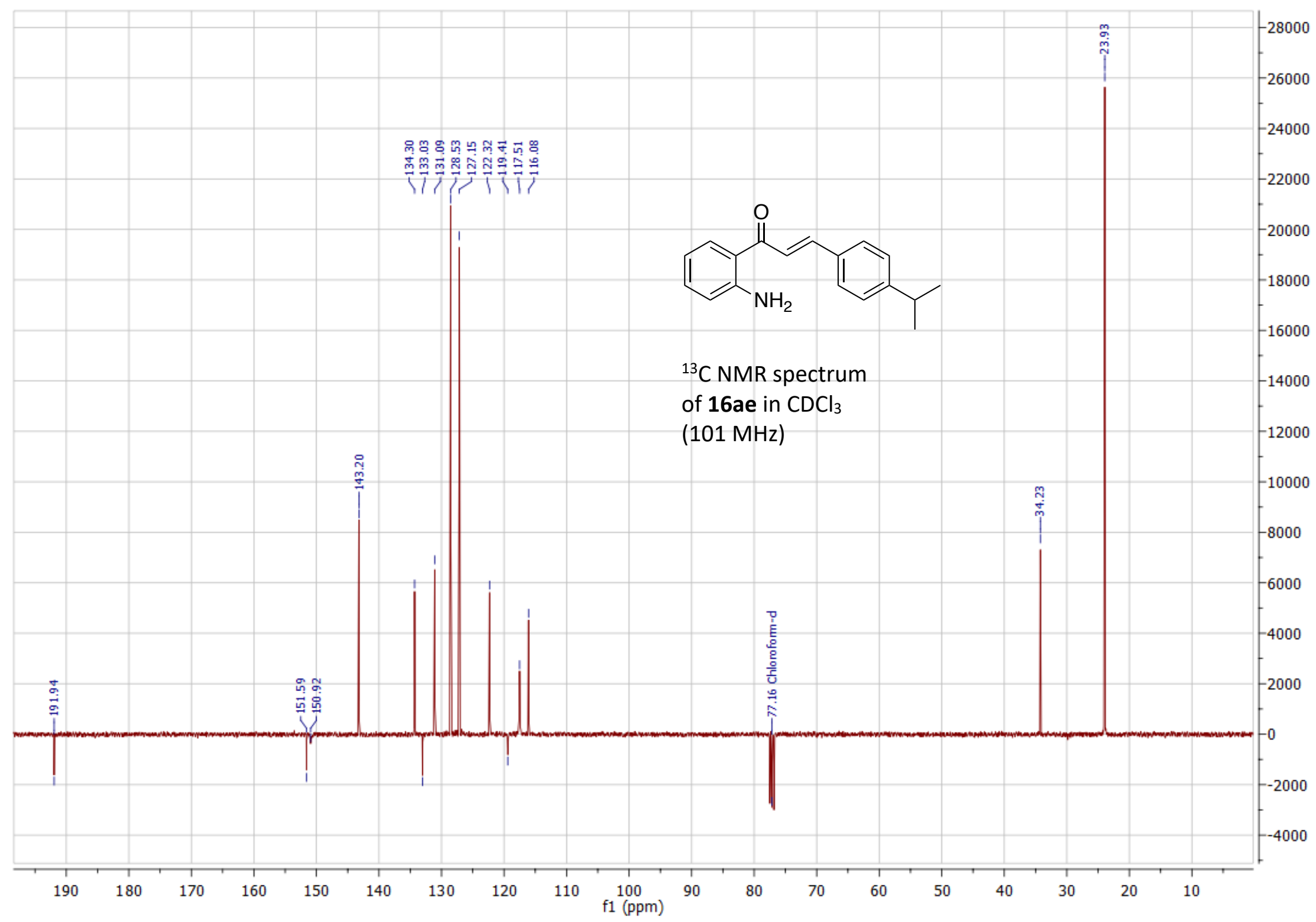




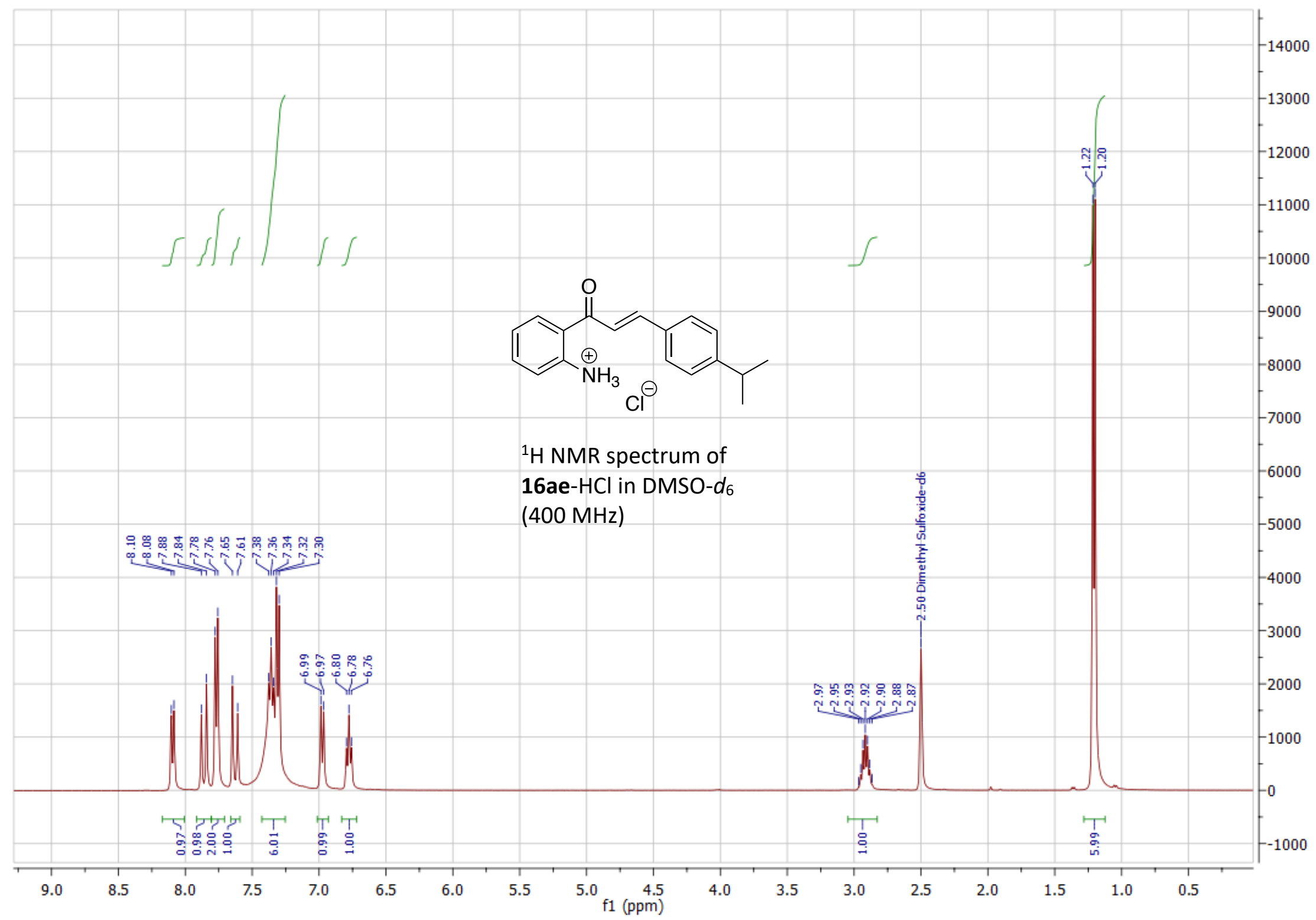




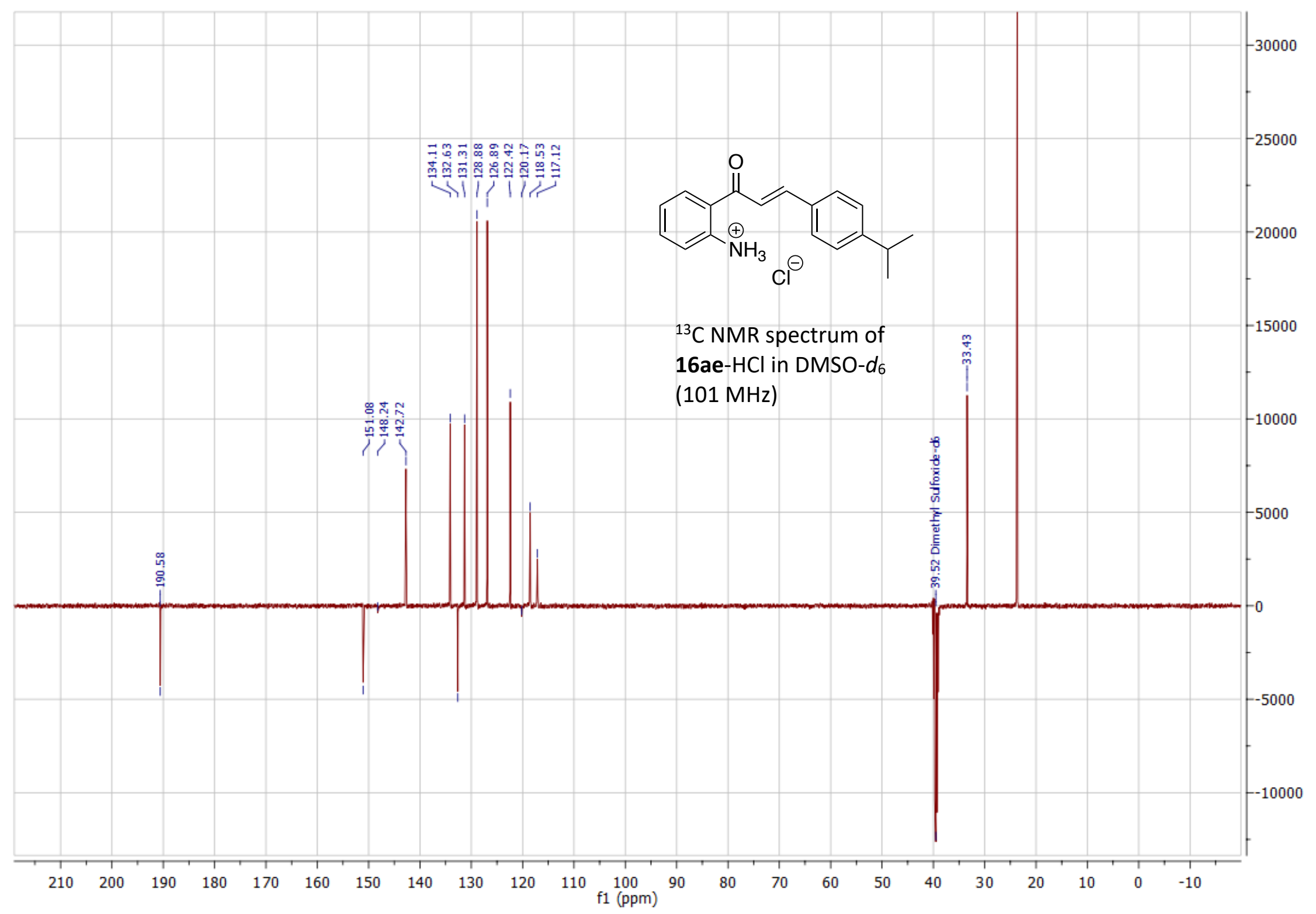




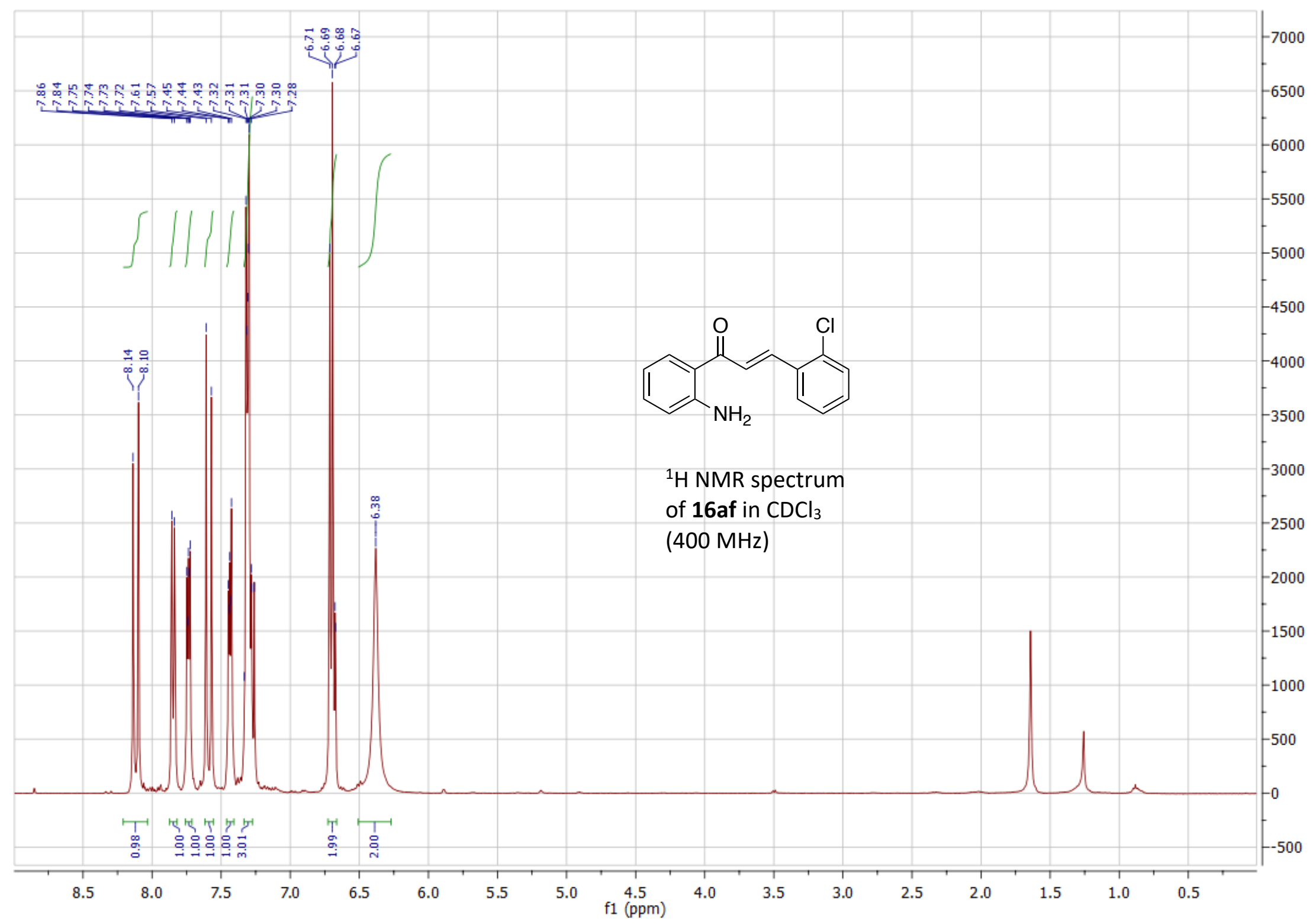




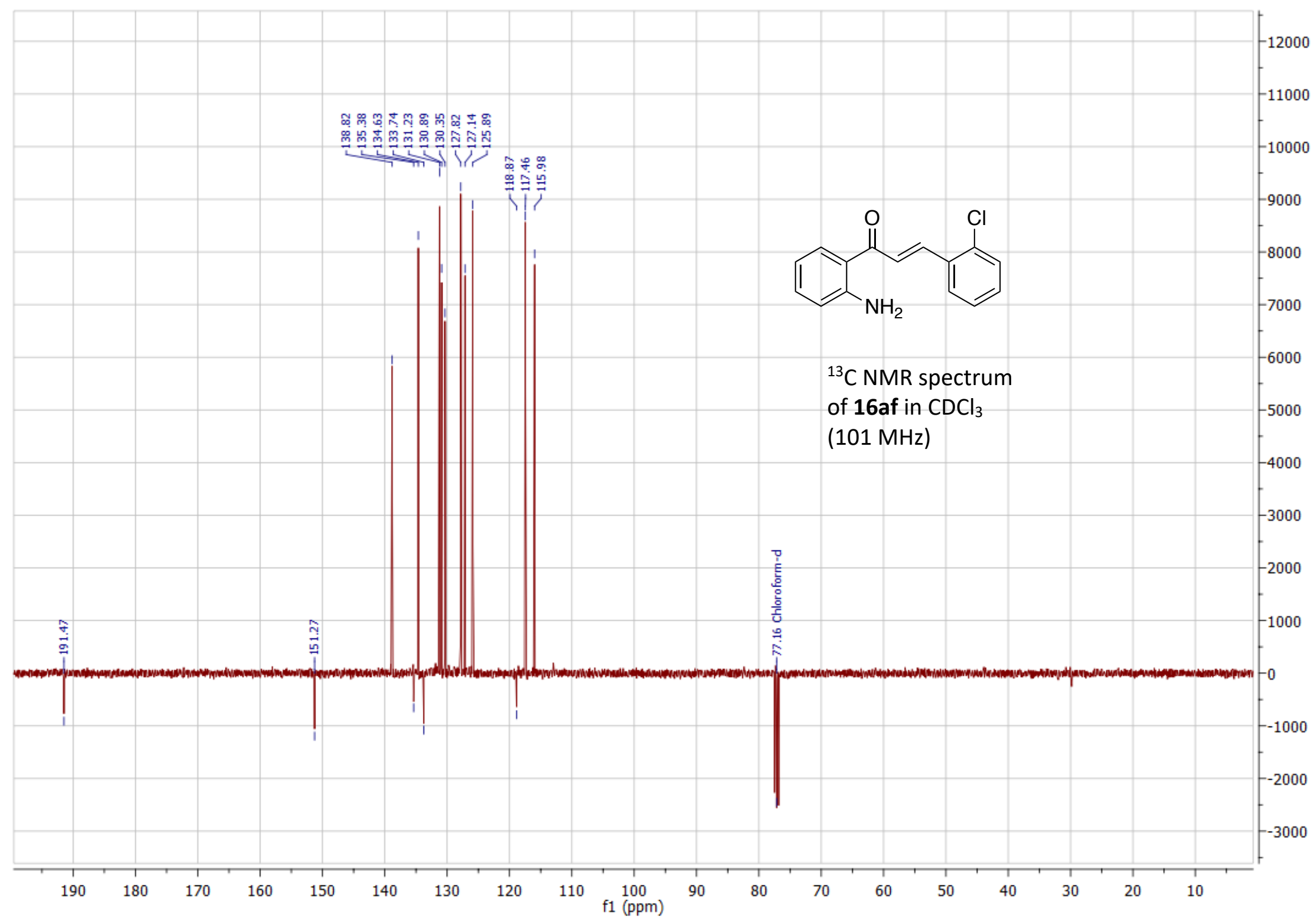




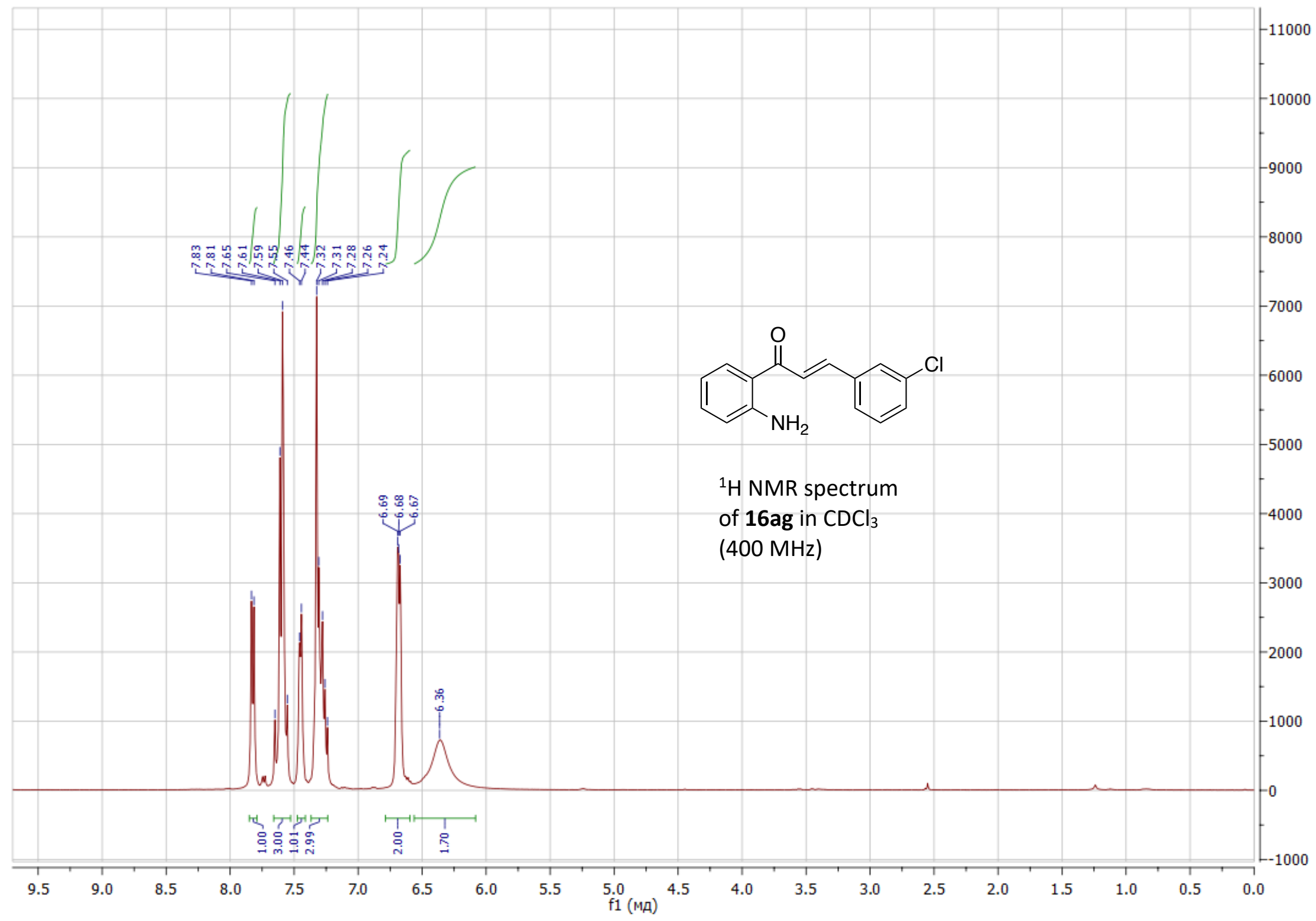




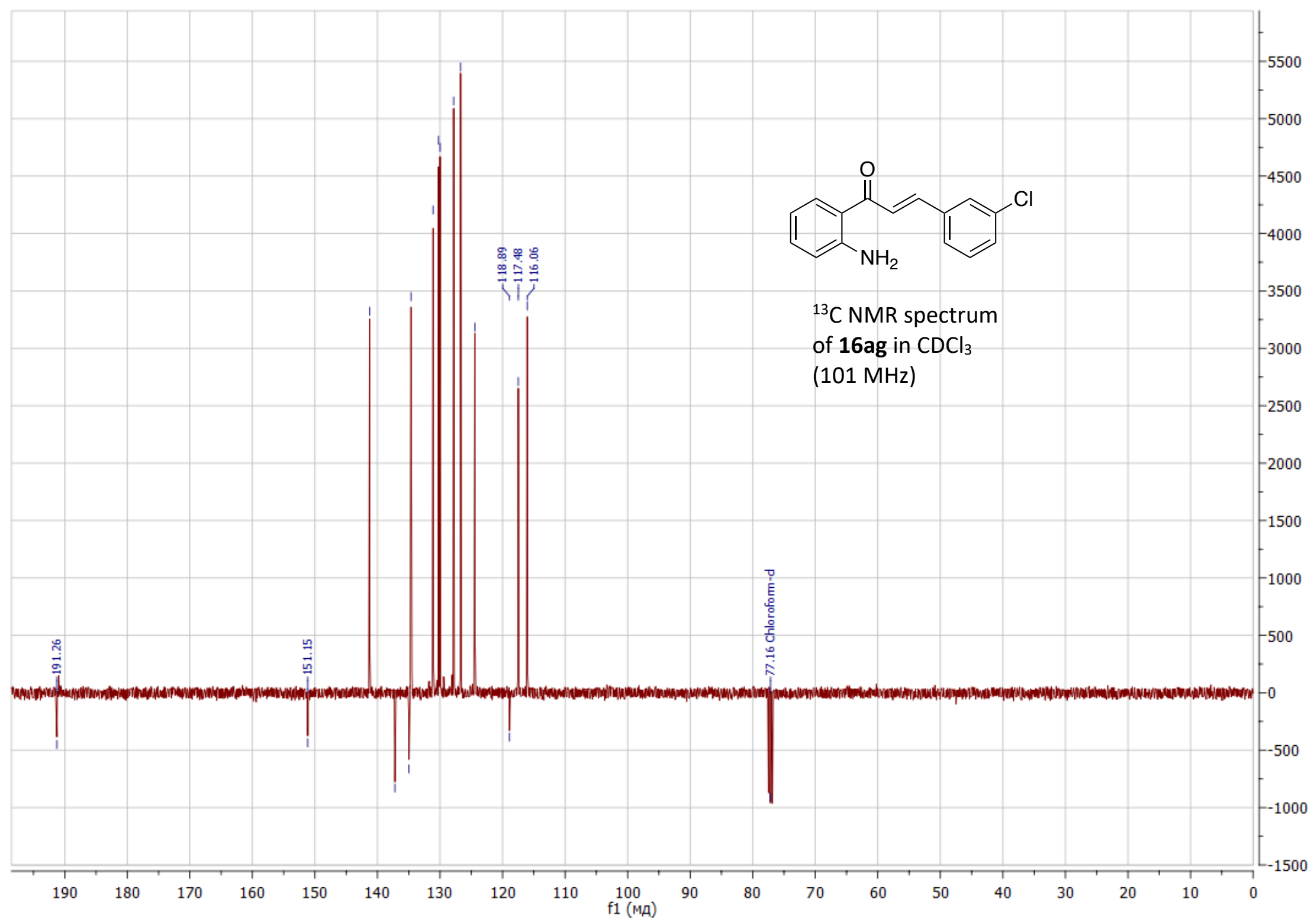




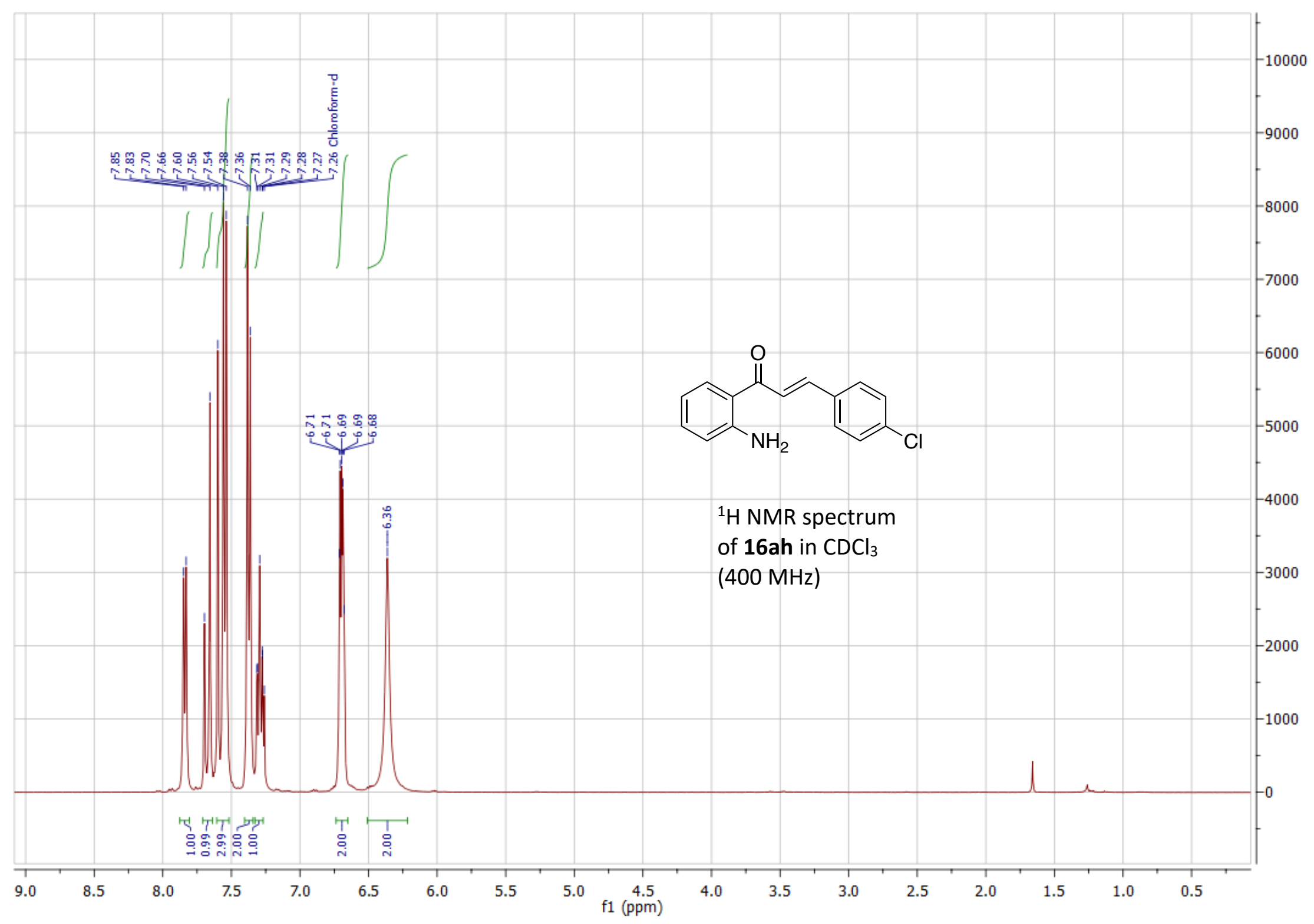




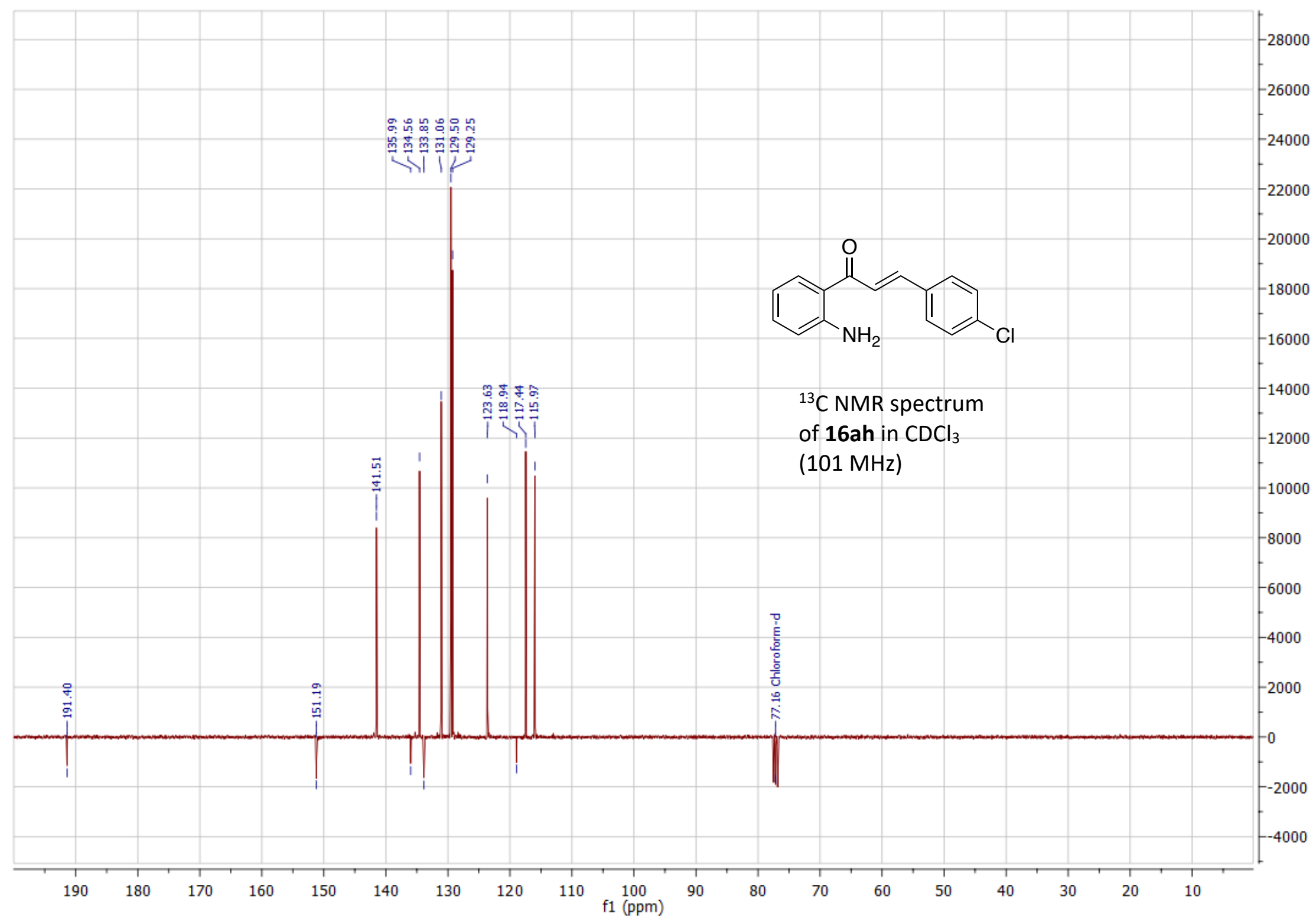




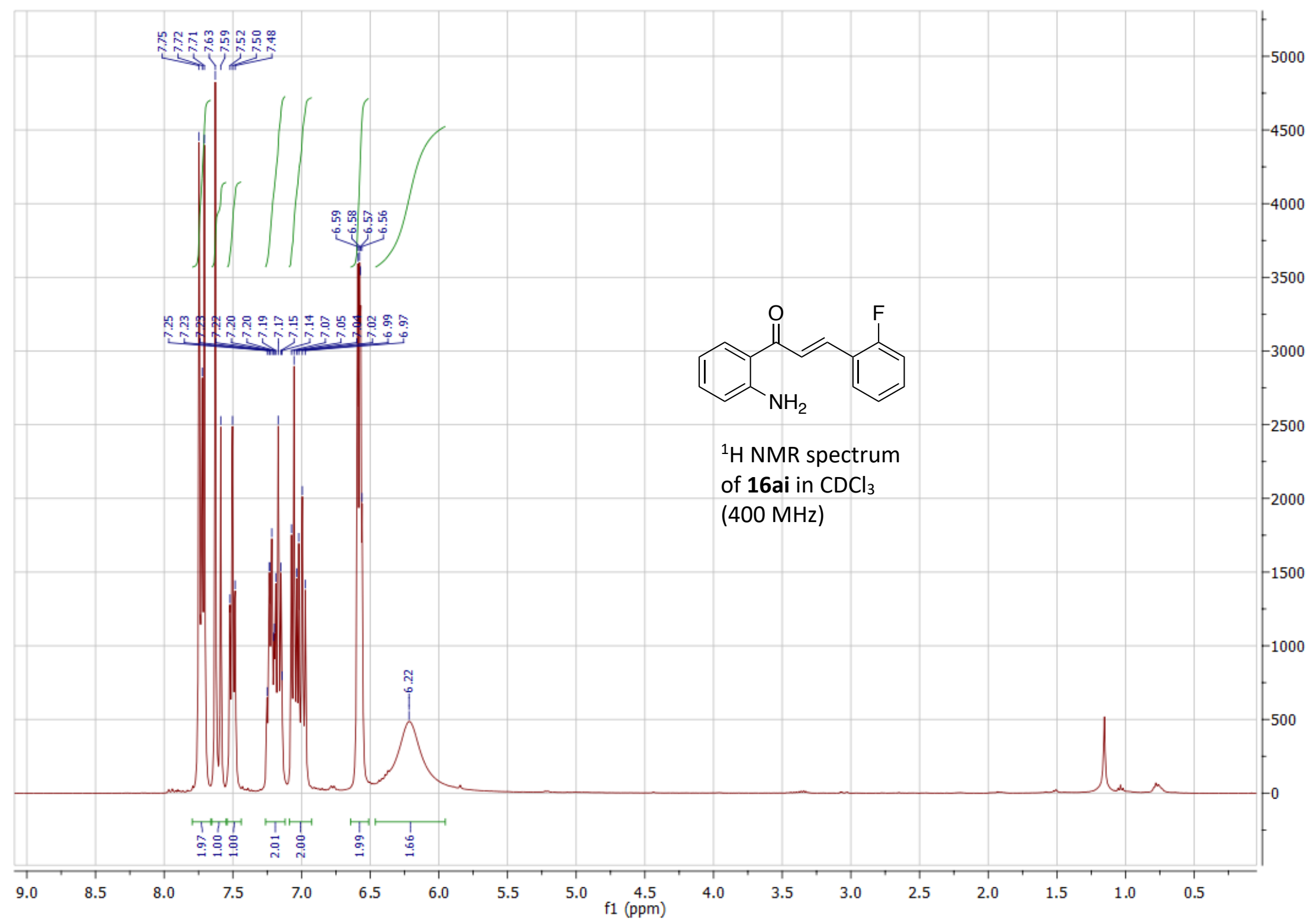




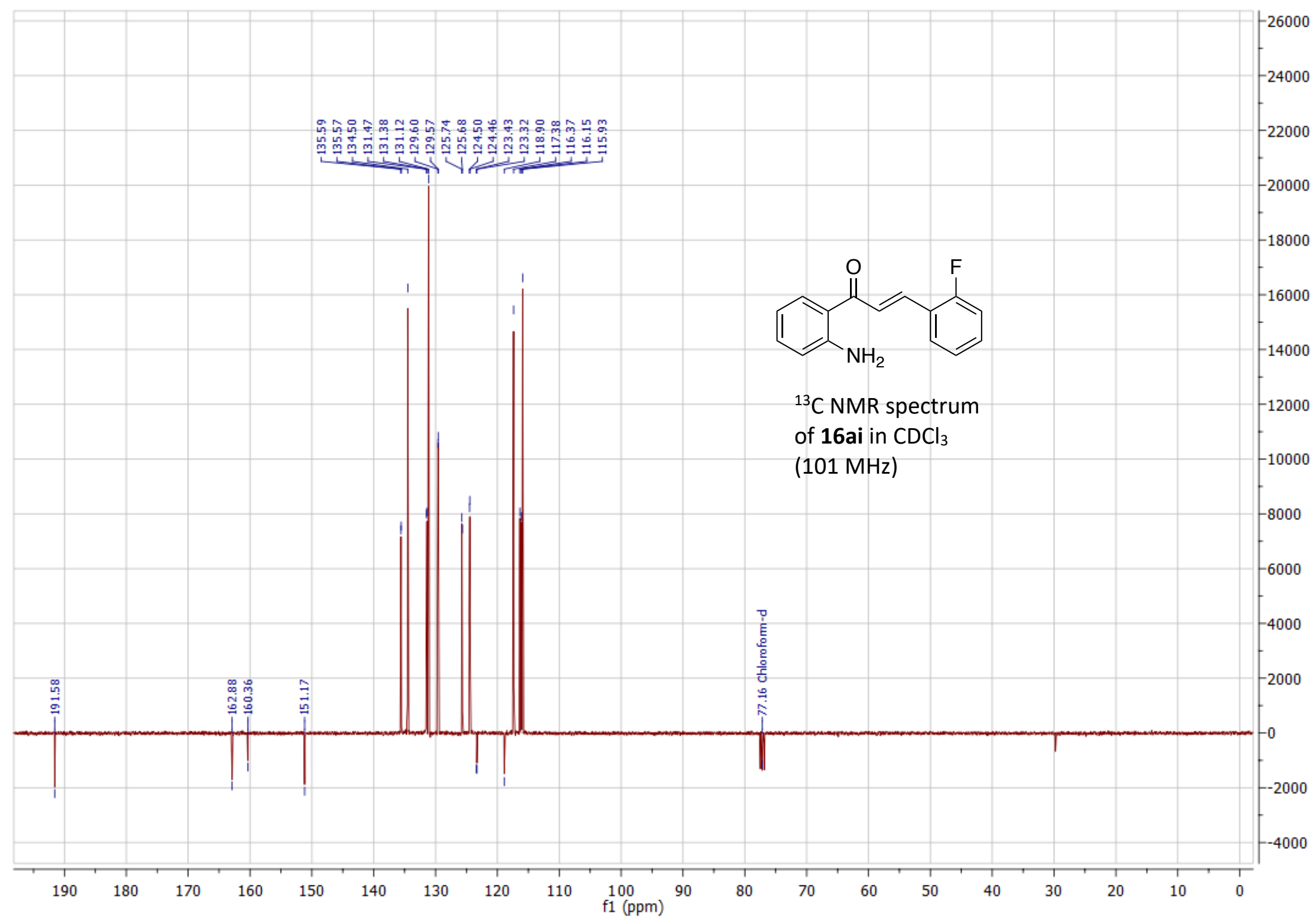




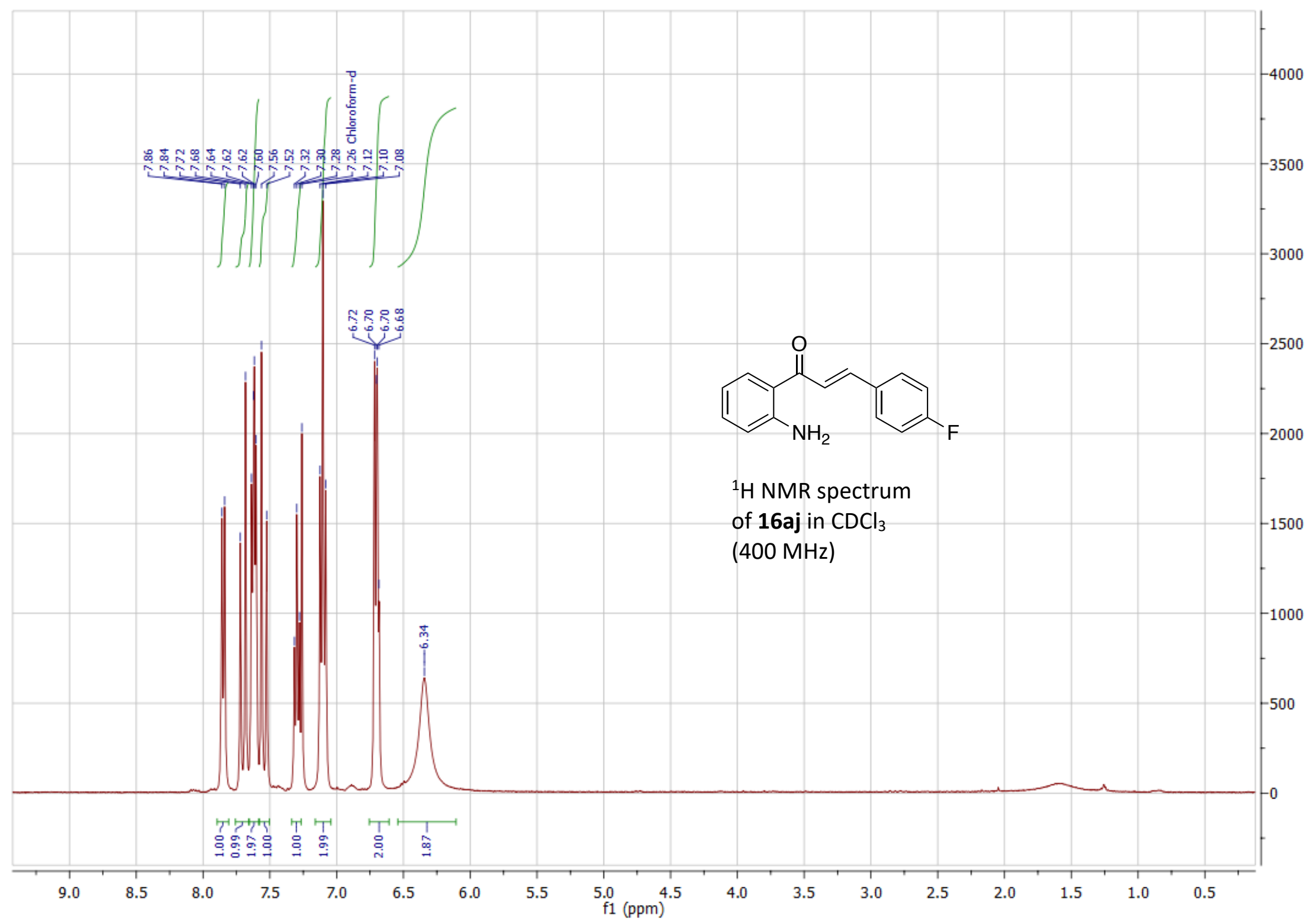




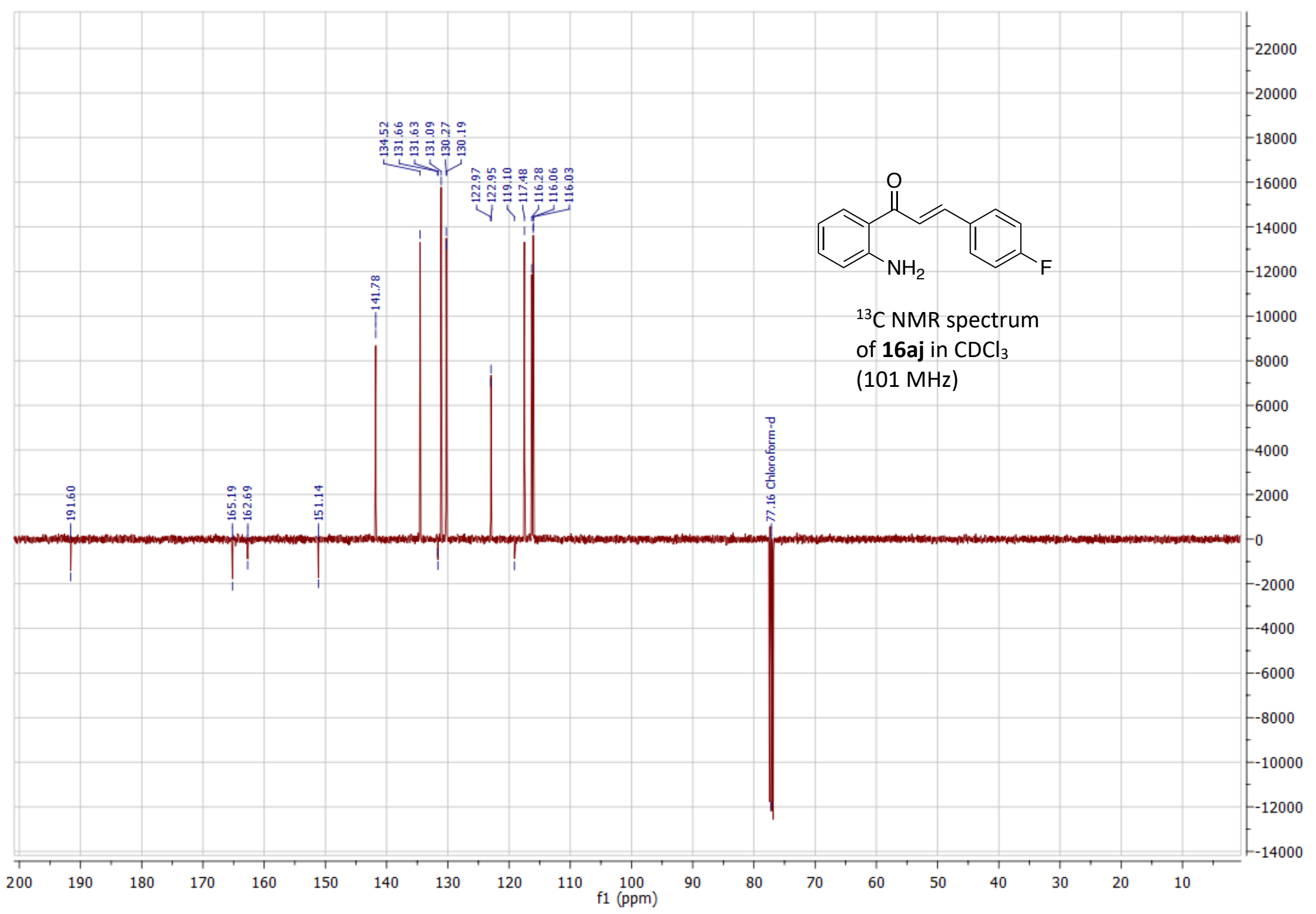




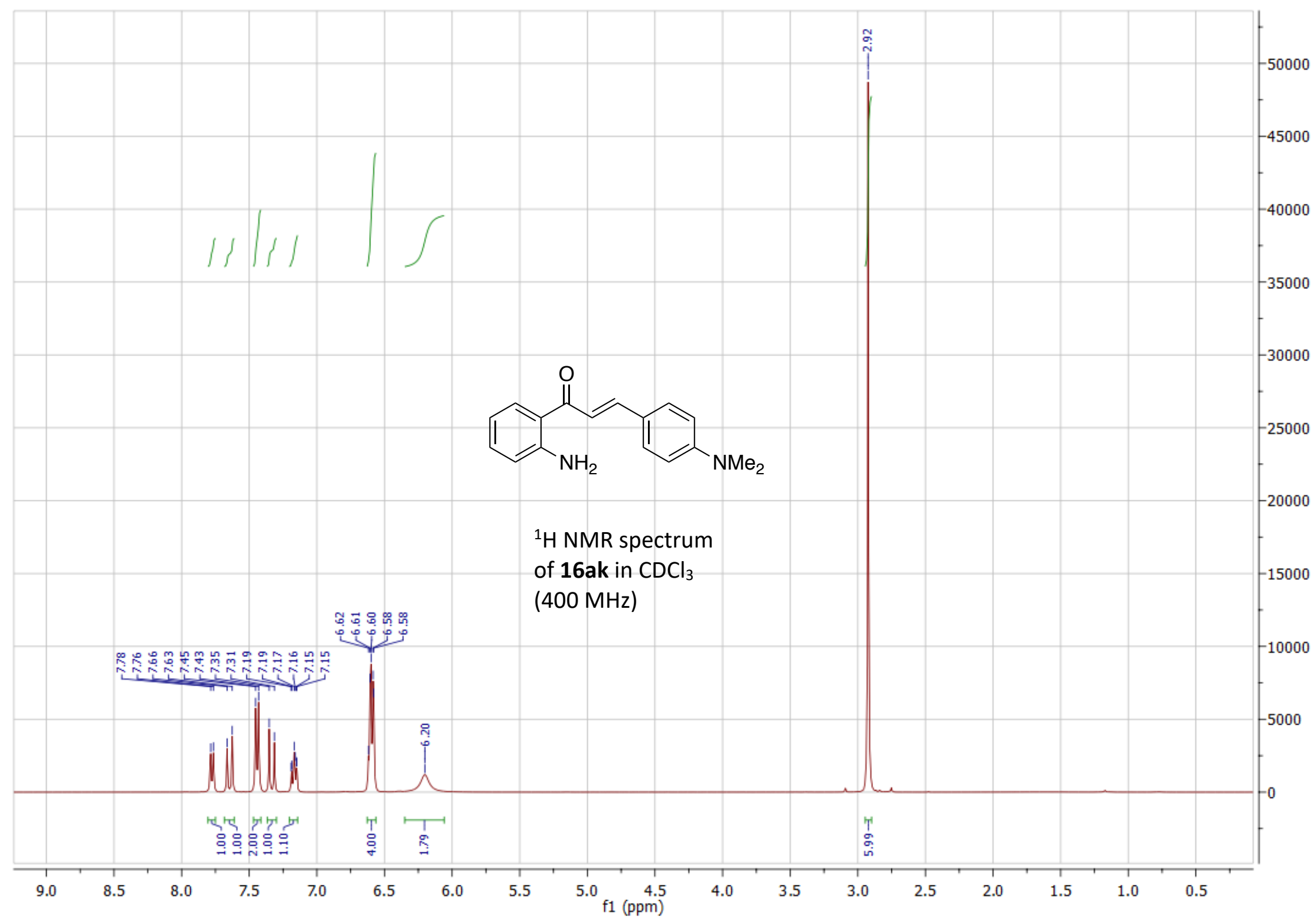




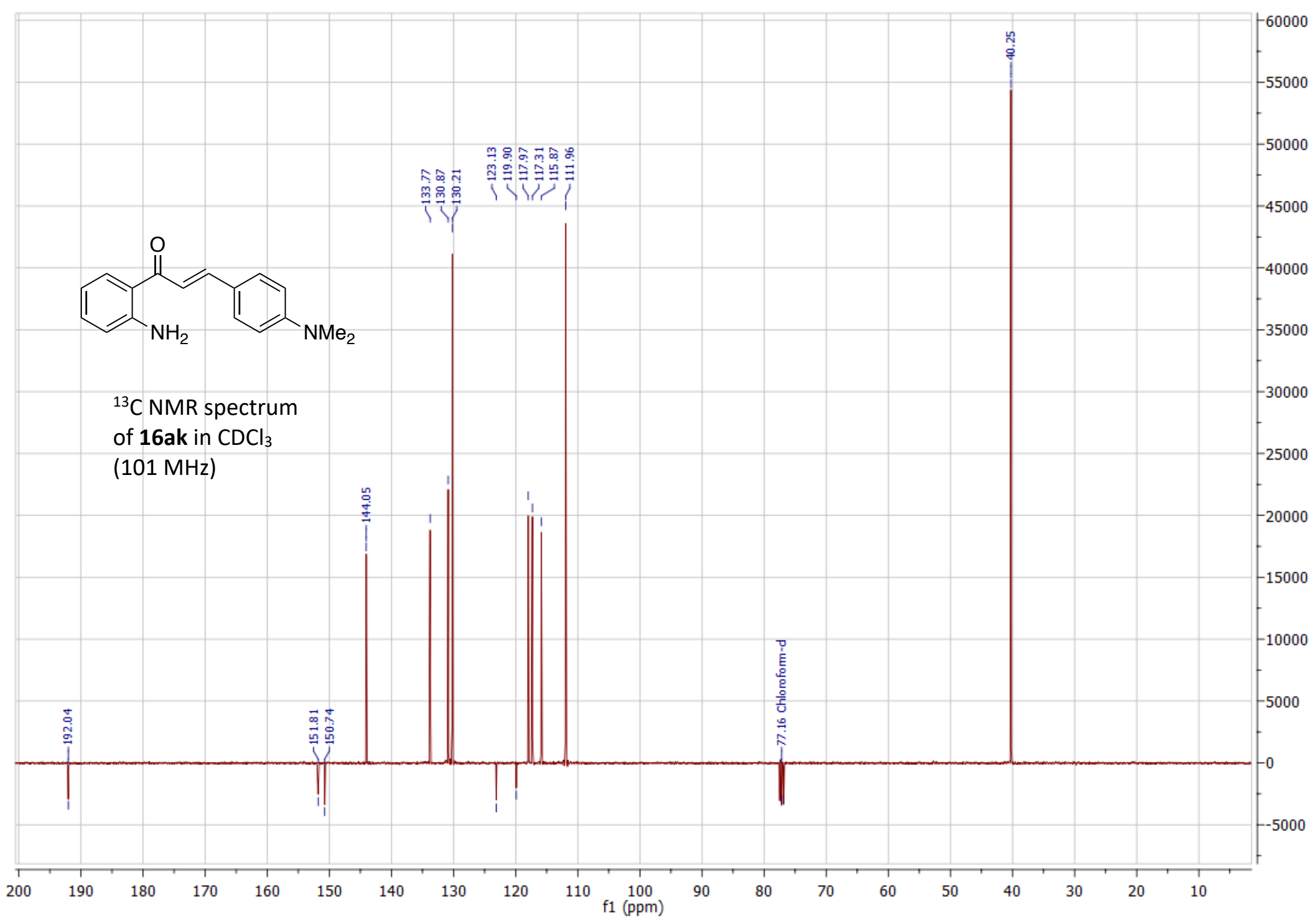




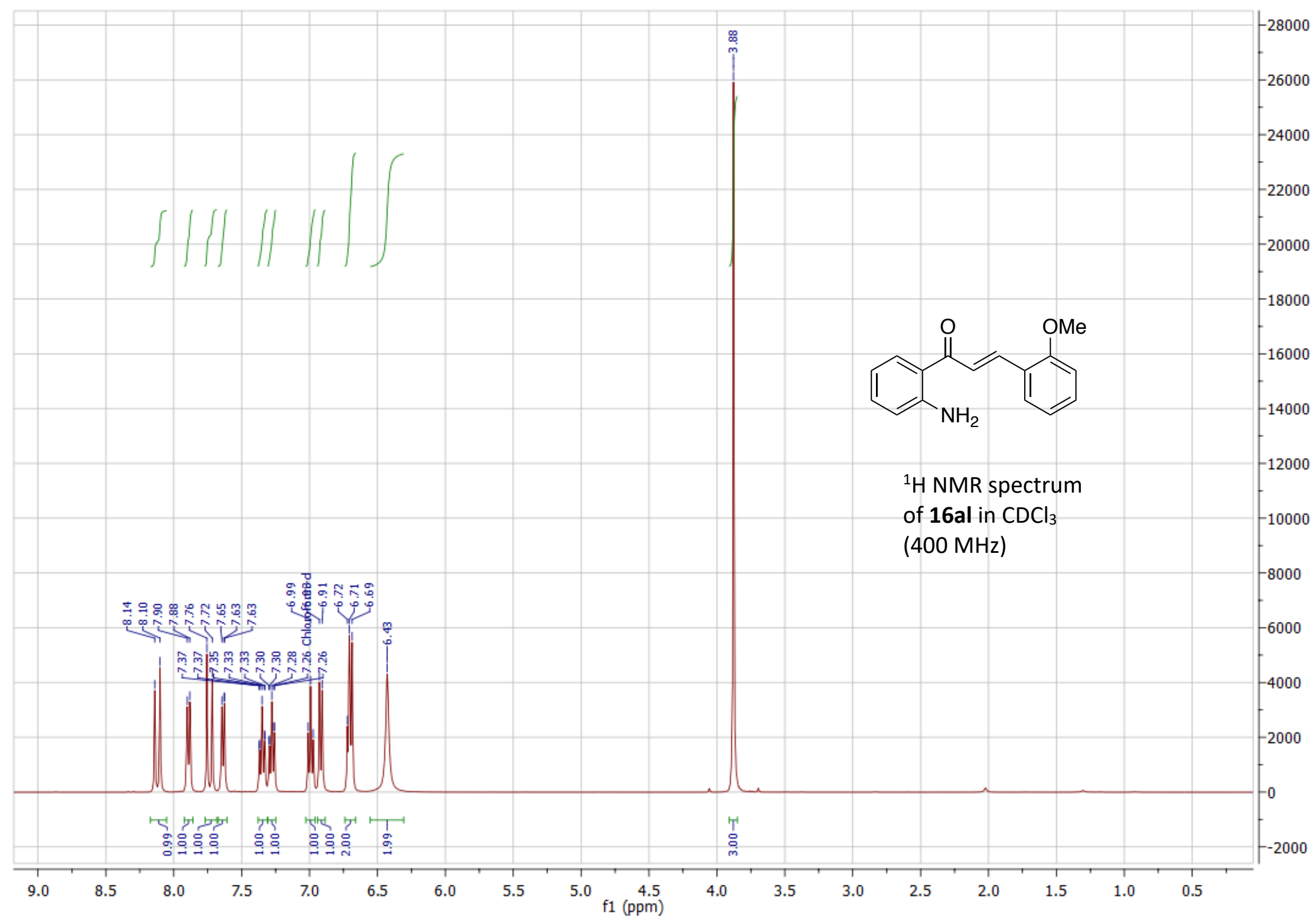




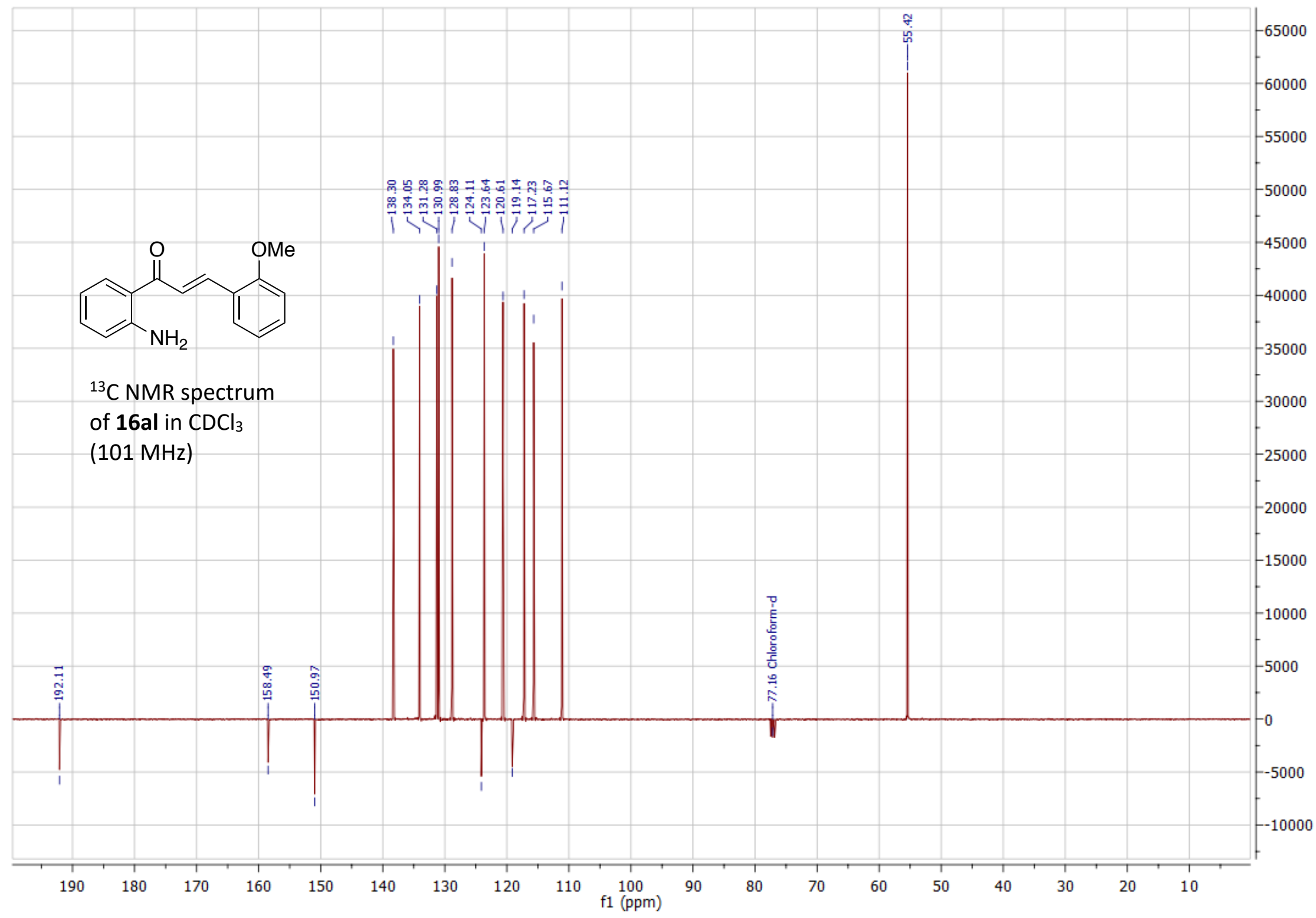




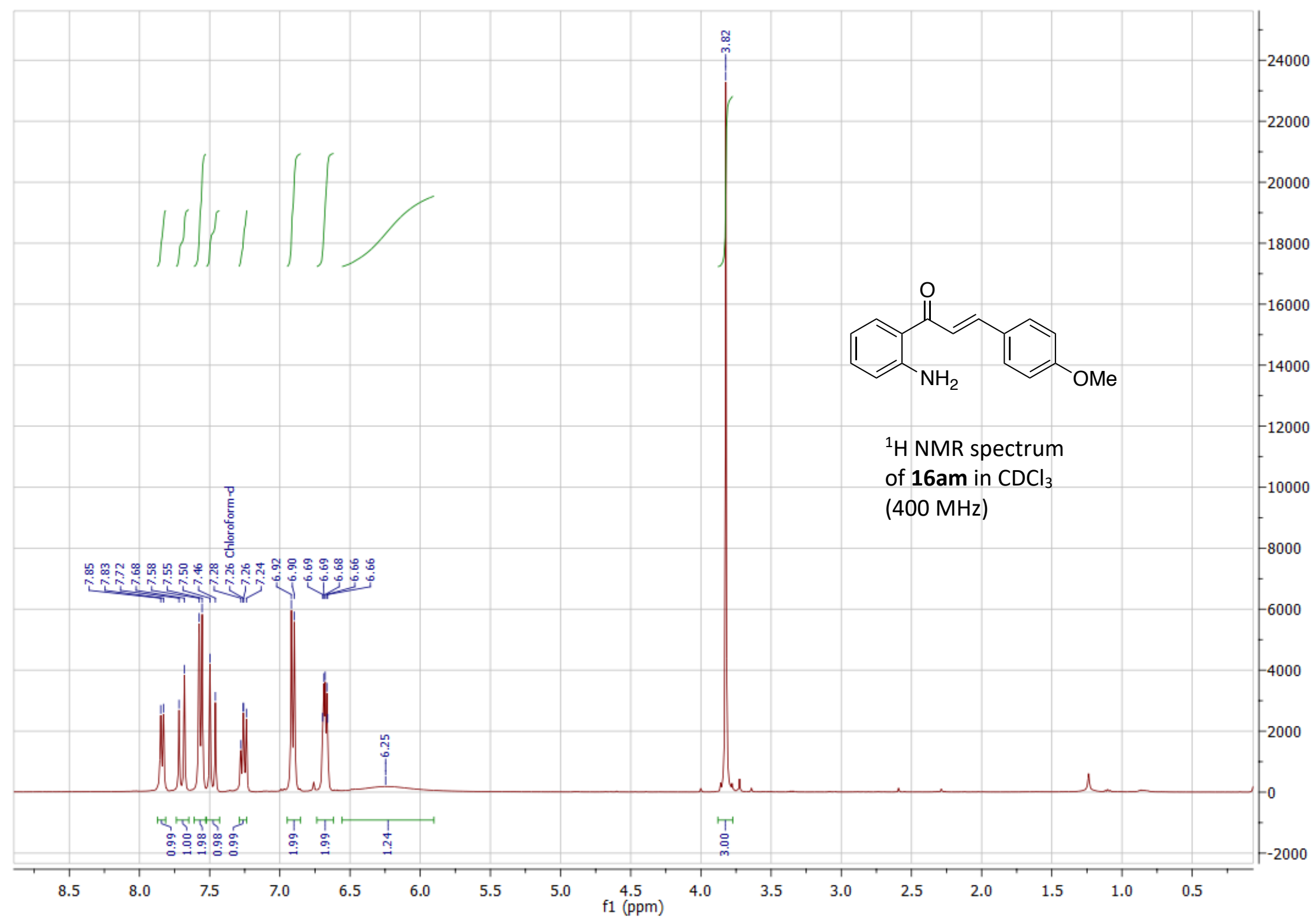




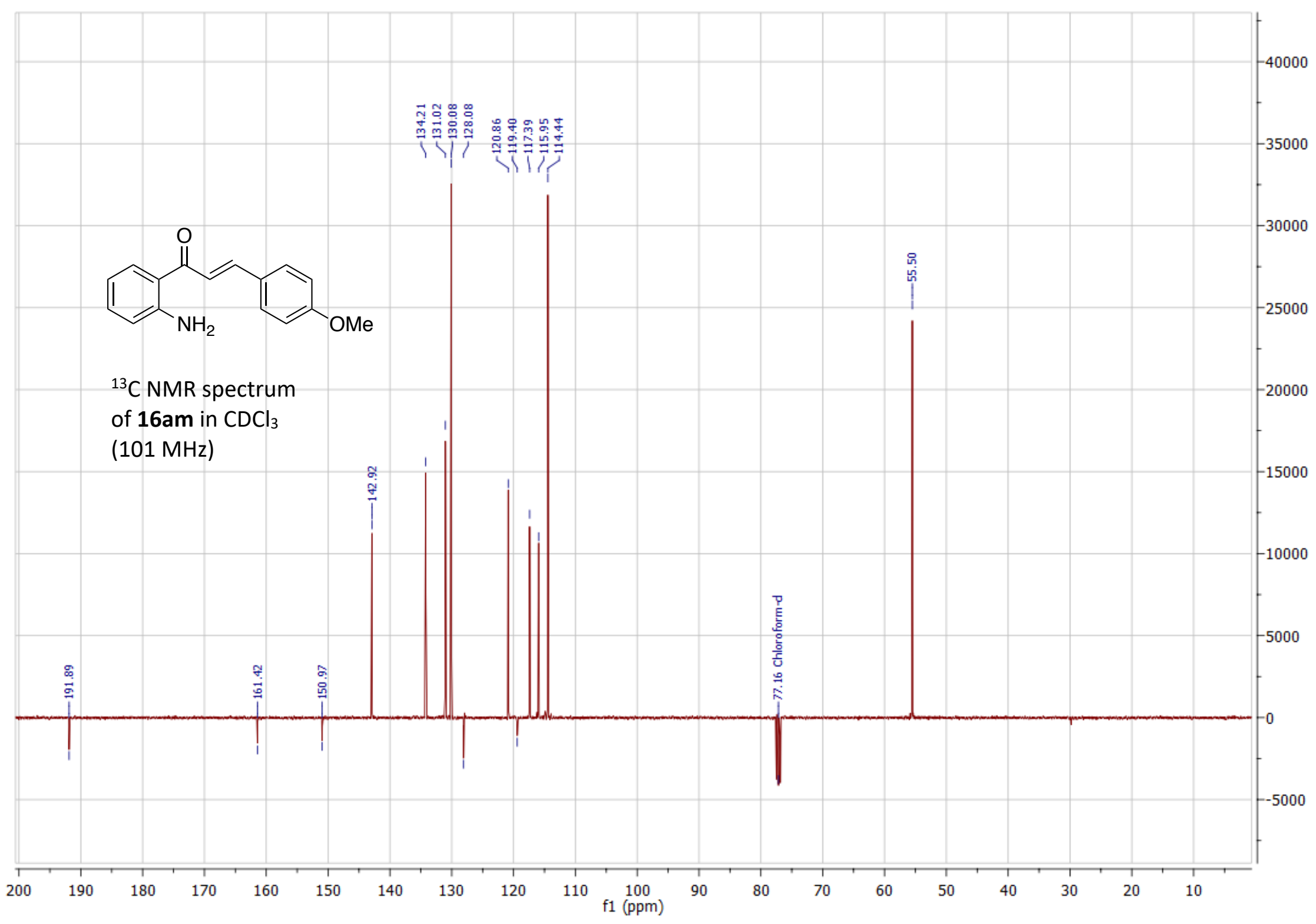




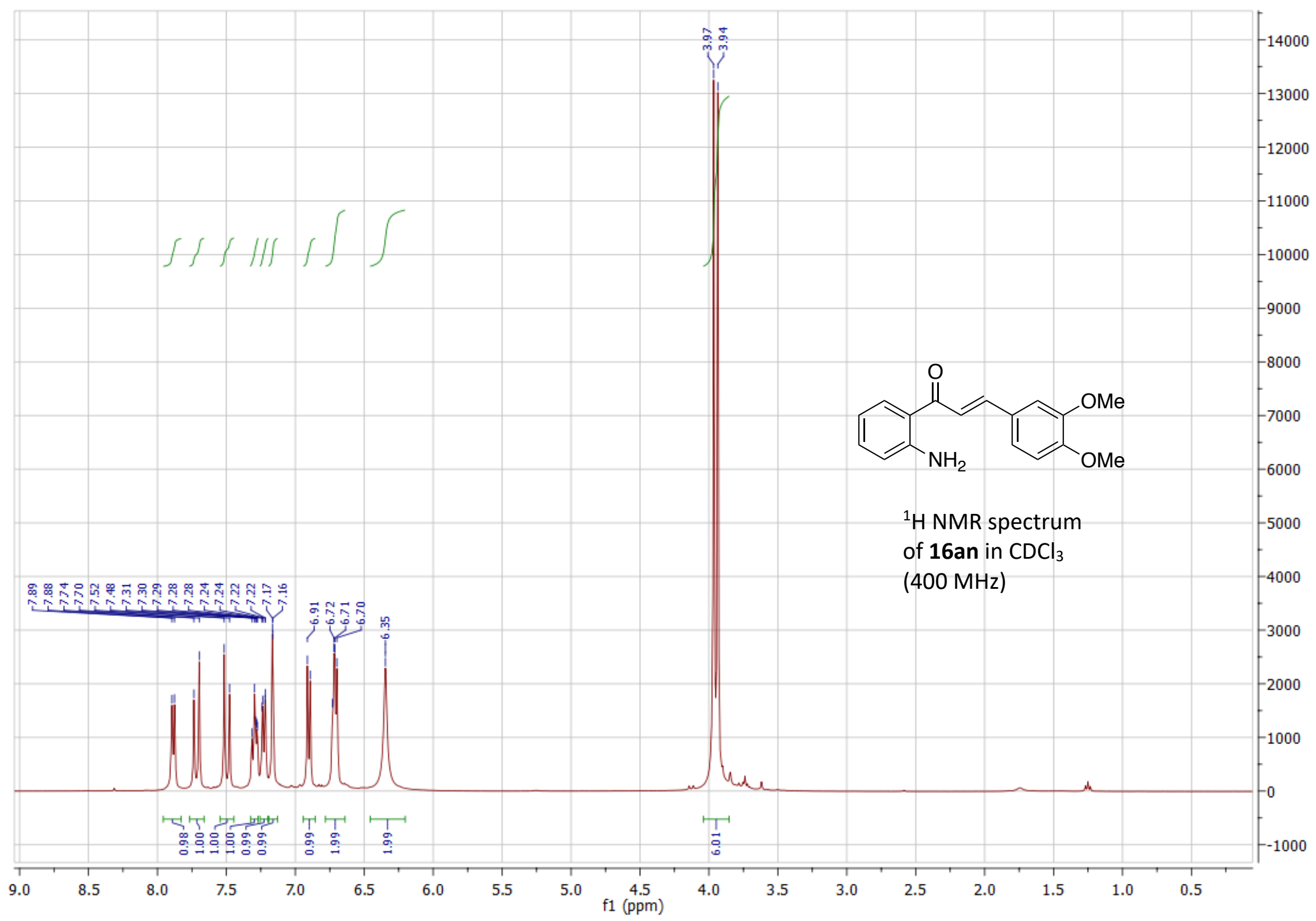




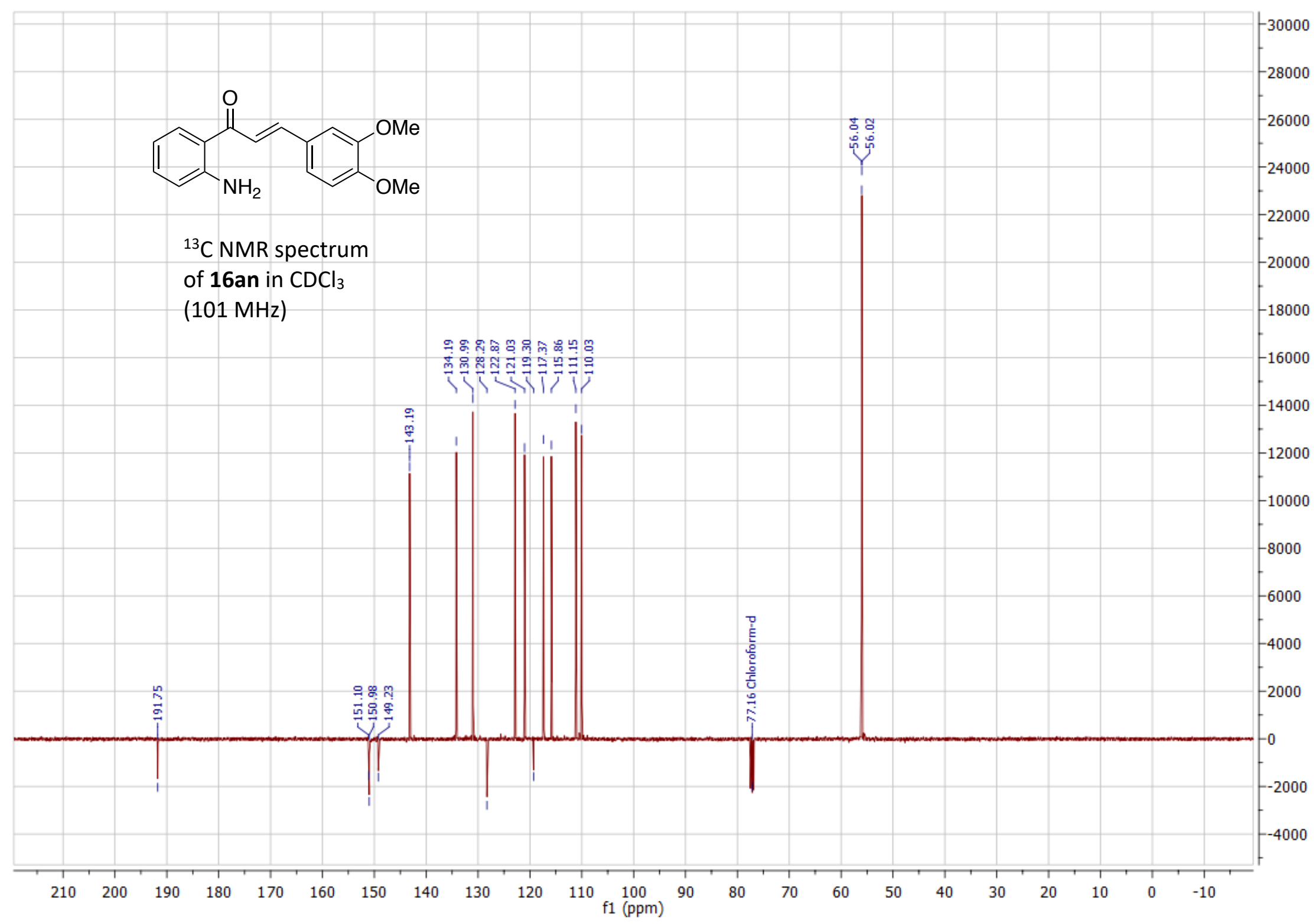




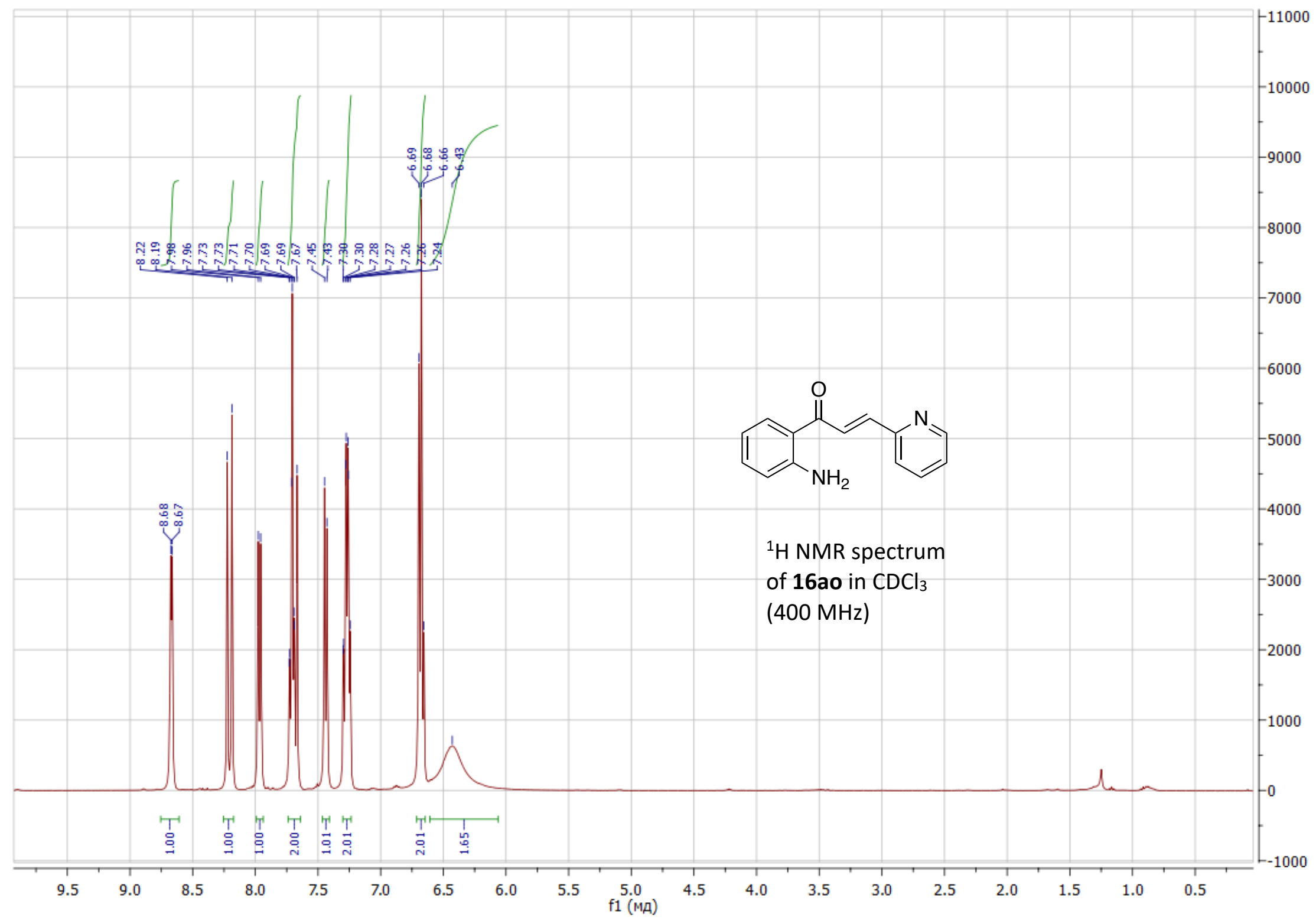




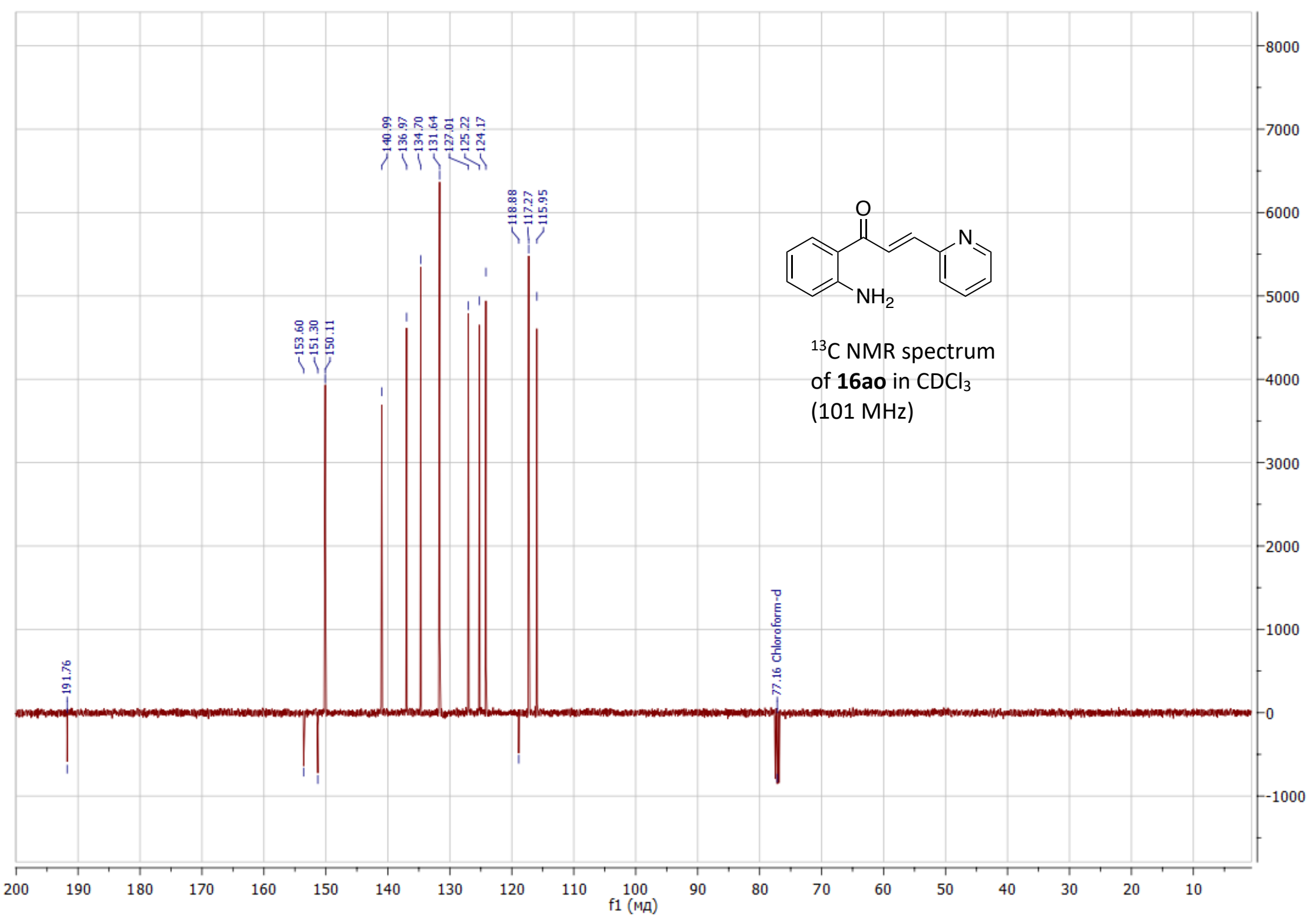




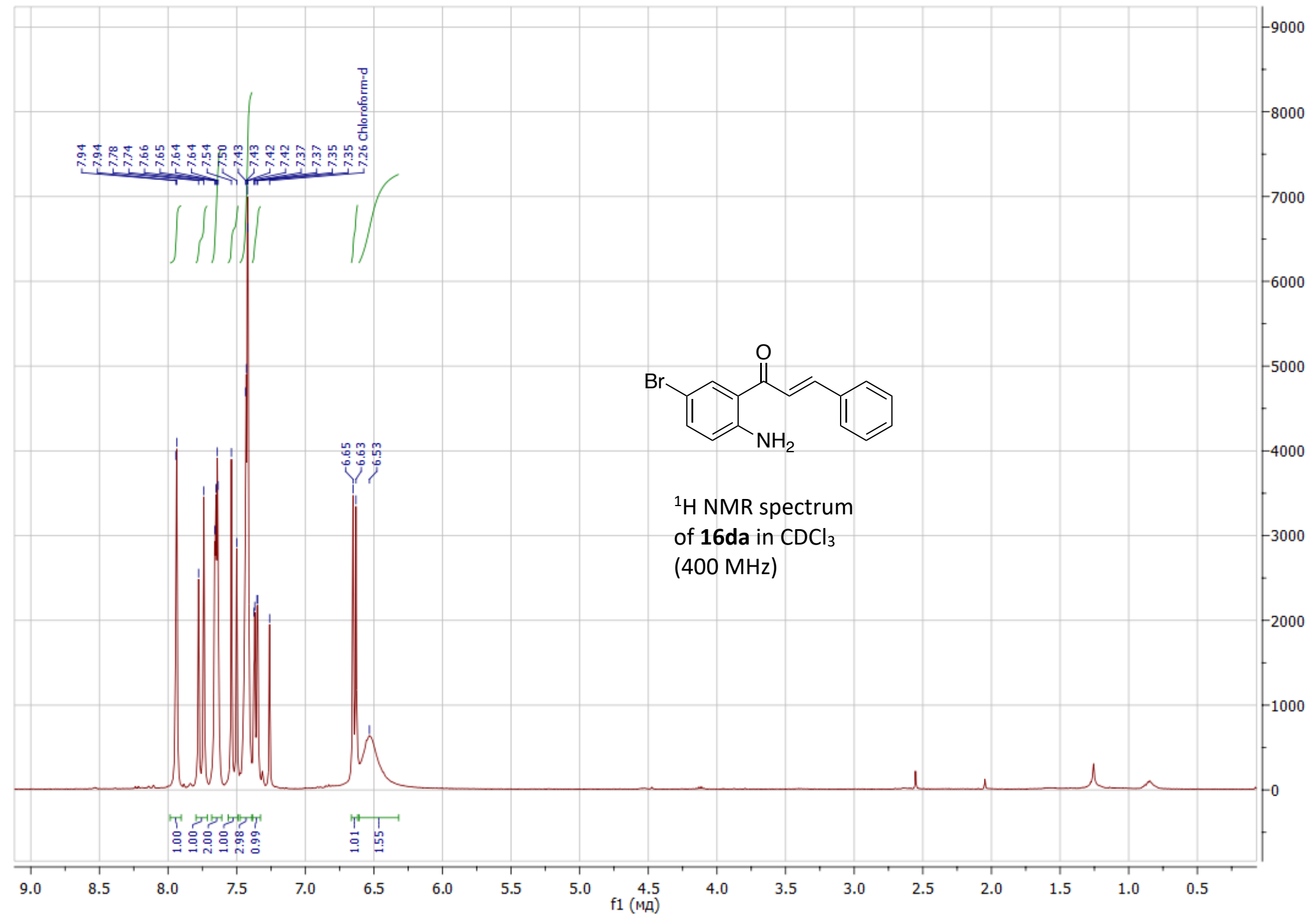




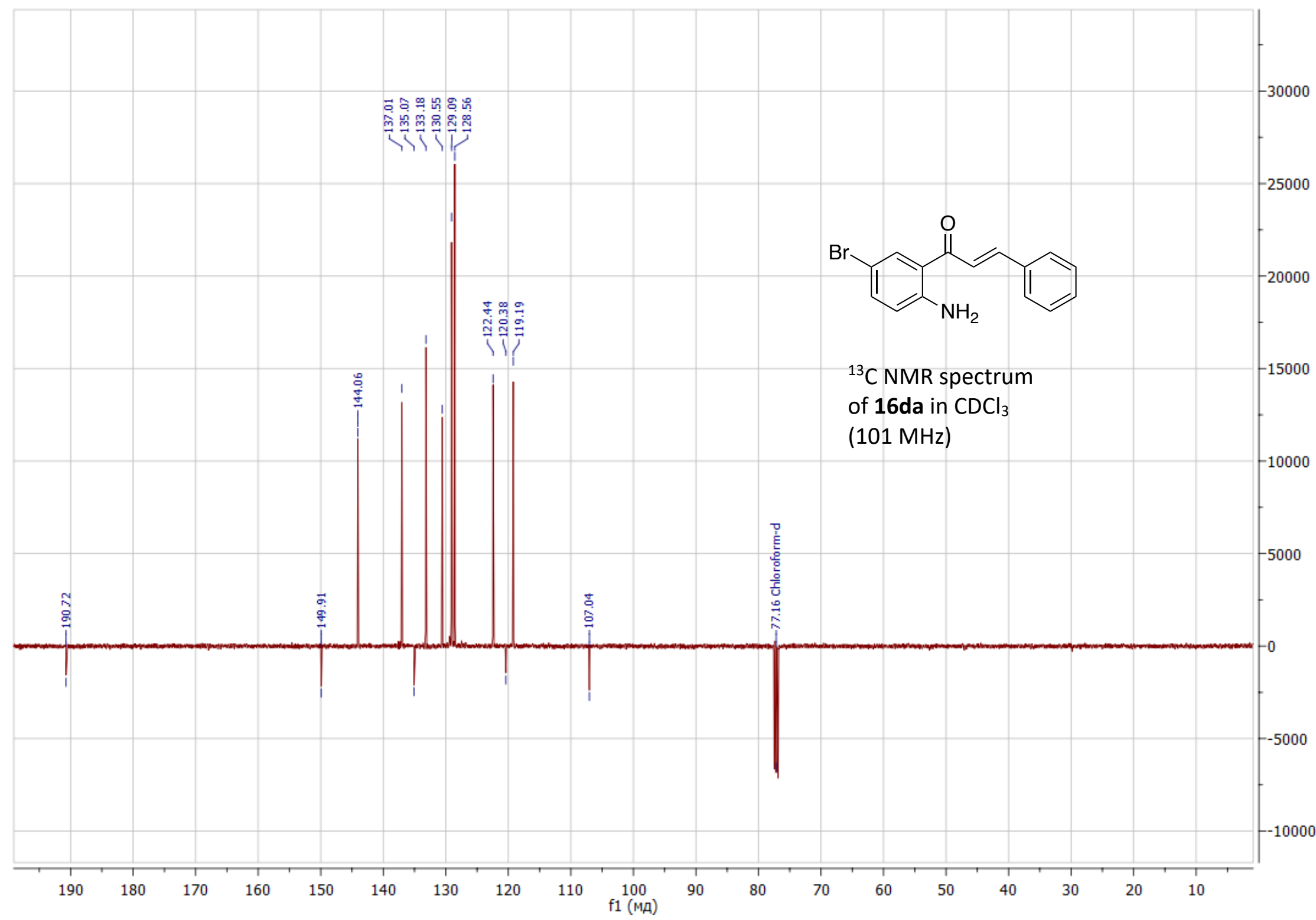


${ }^{1} \mathrm{H}$ and ${ }^{13} \mathrm{C}$ Spectral Charts of (E)-1-(2-nitrophenyl)-3-arylprop-2-en-1-ones 20

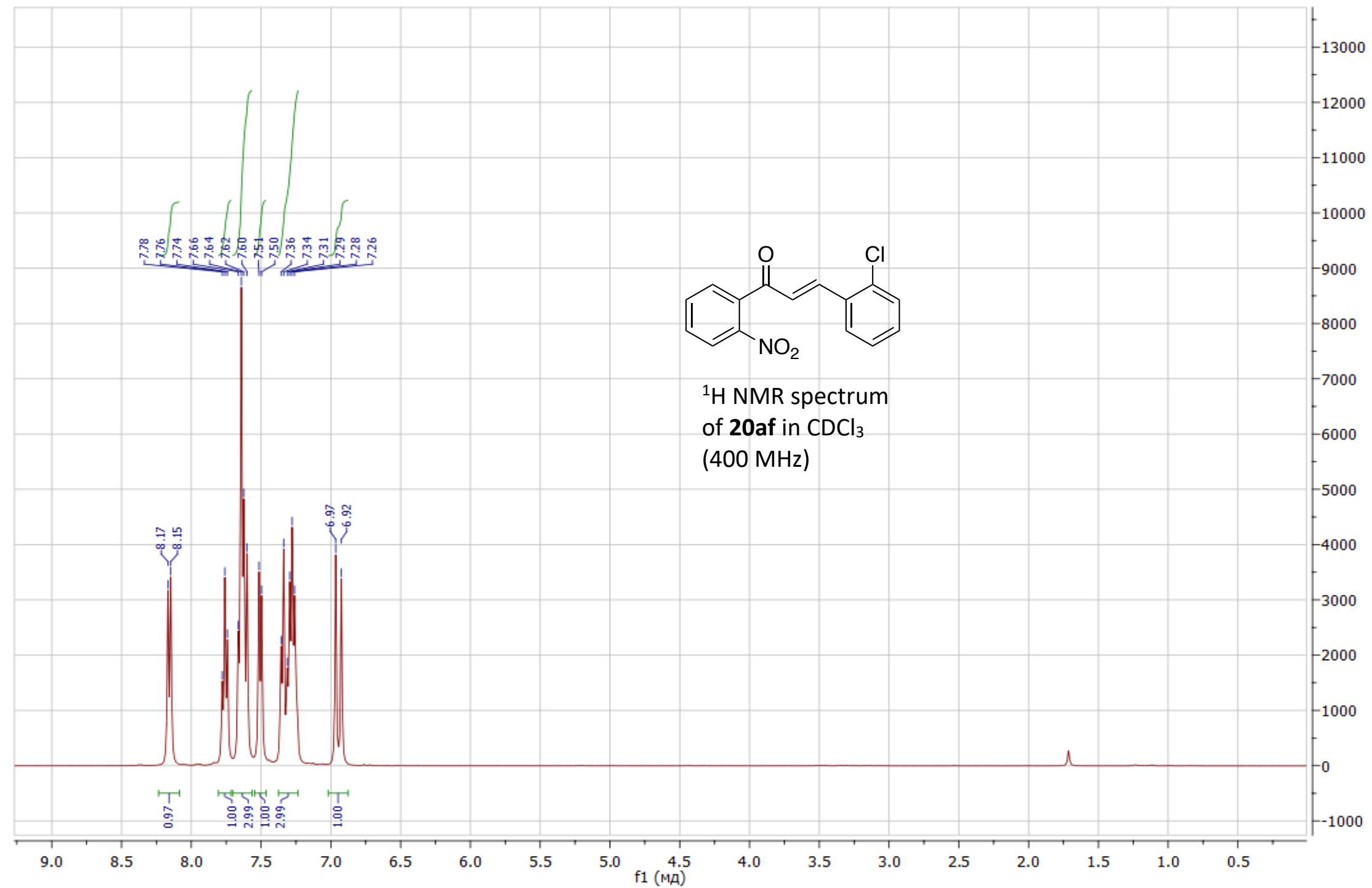




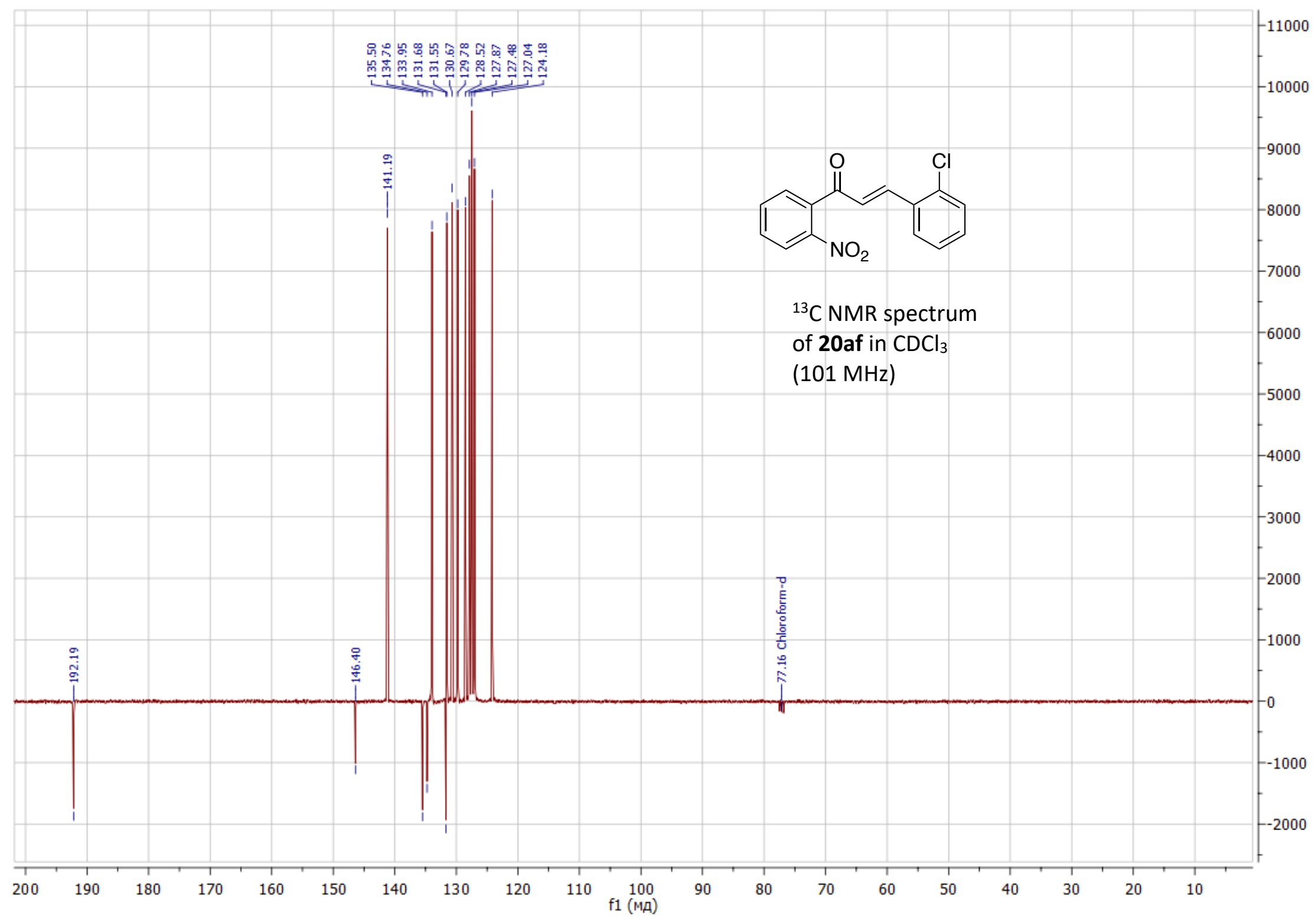




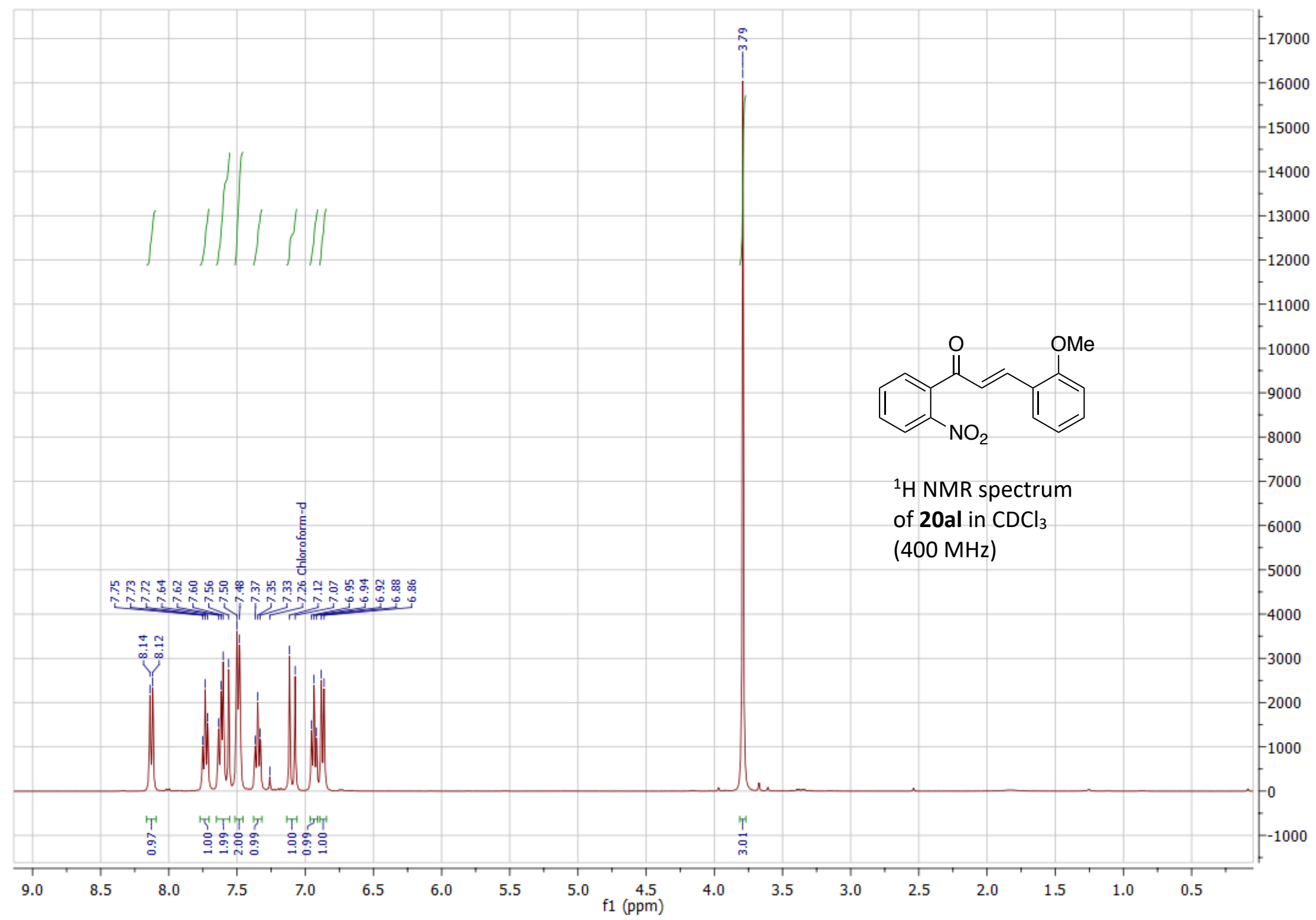




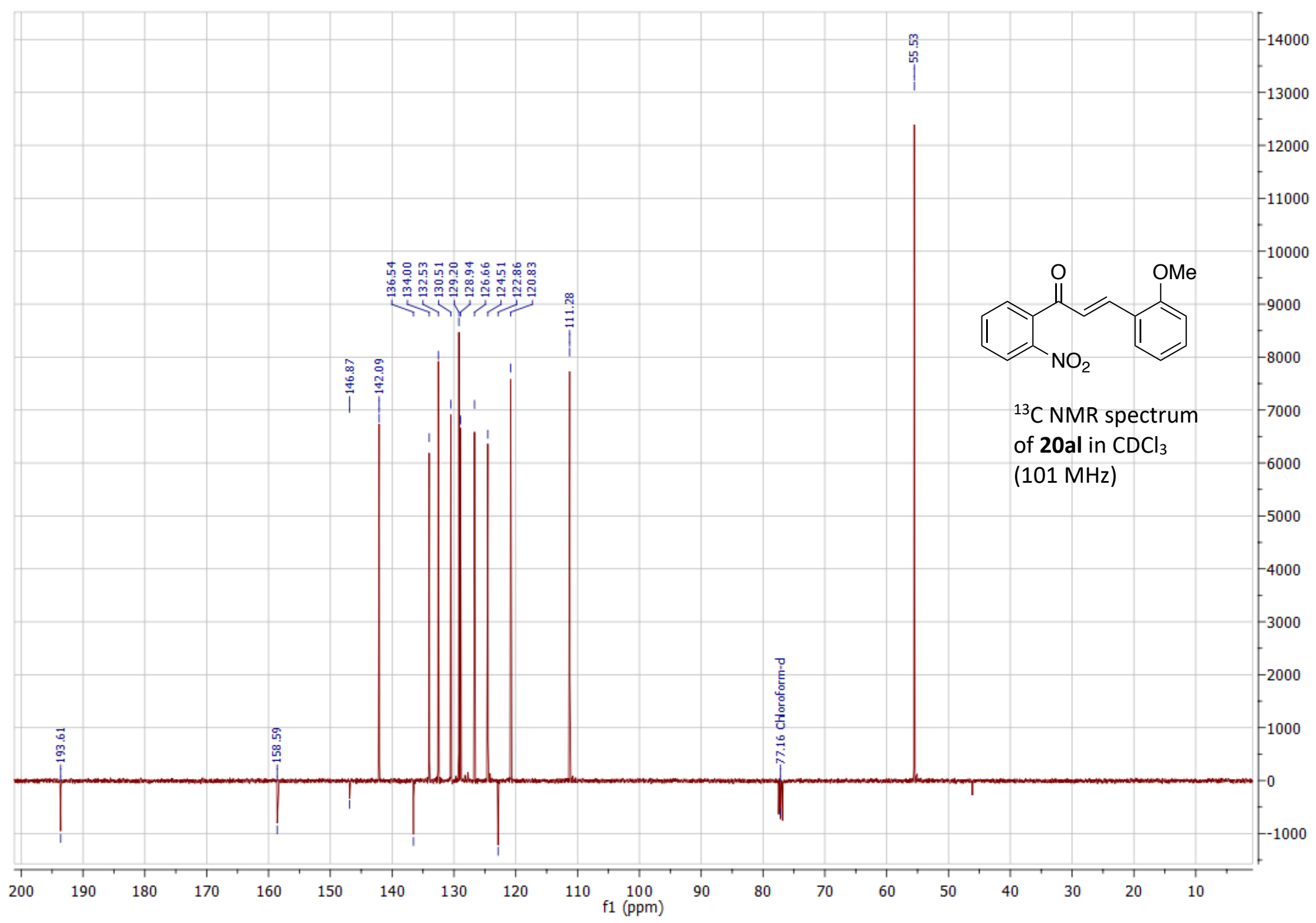




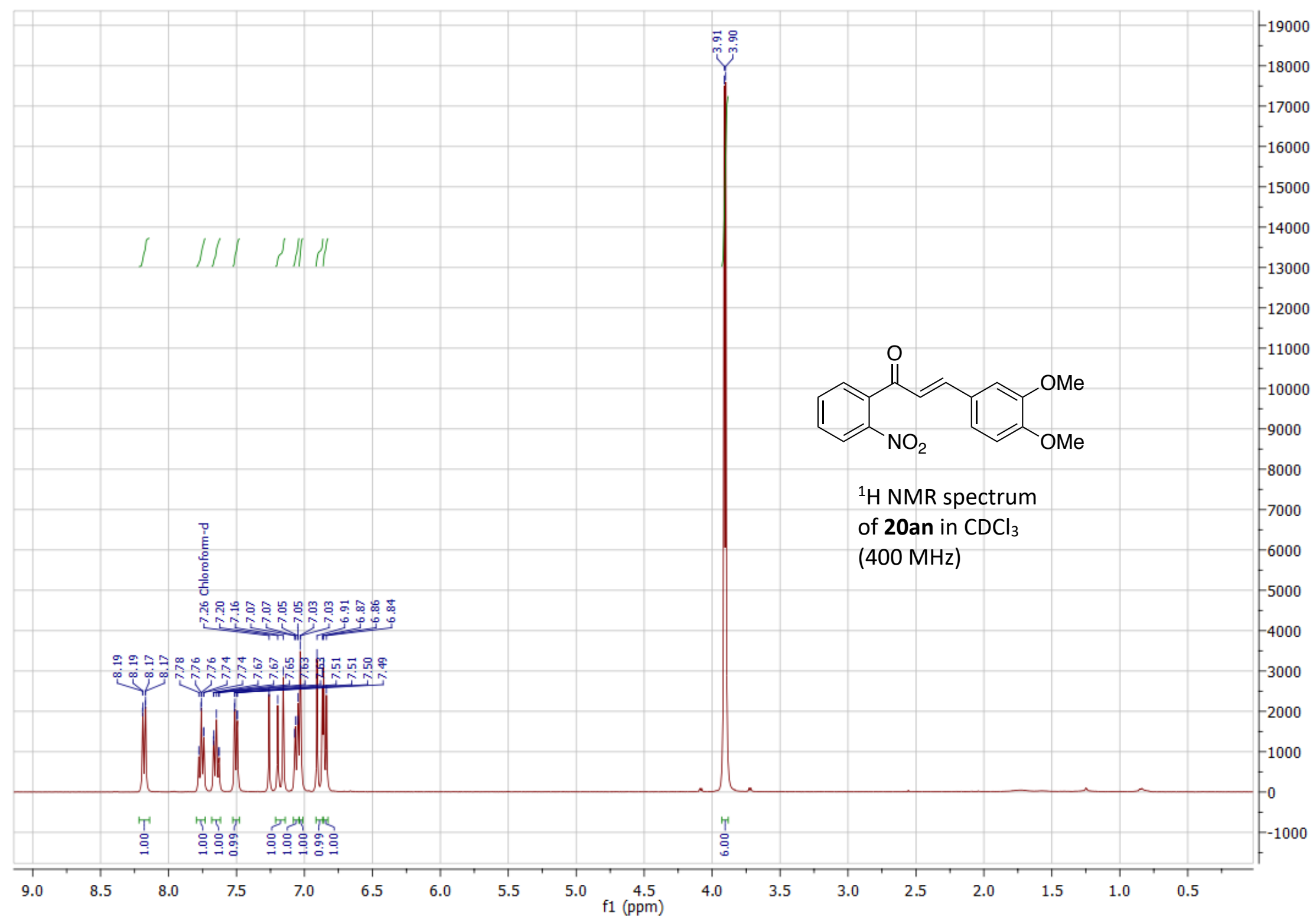




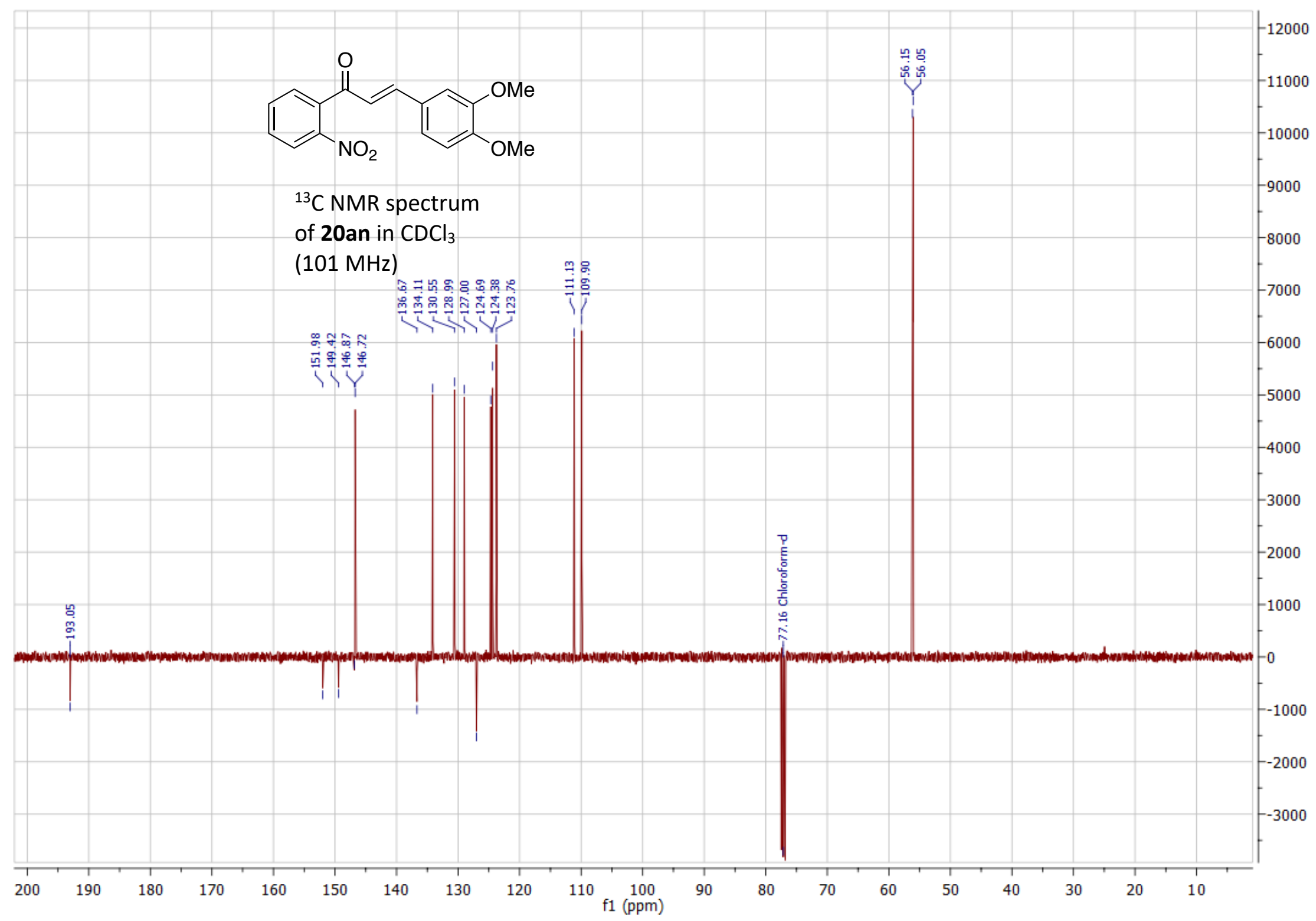




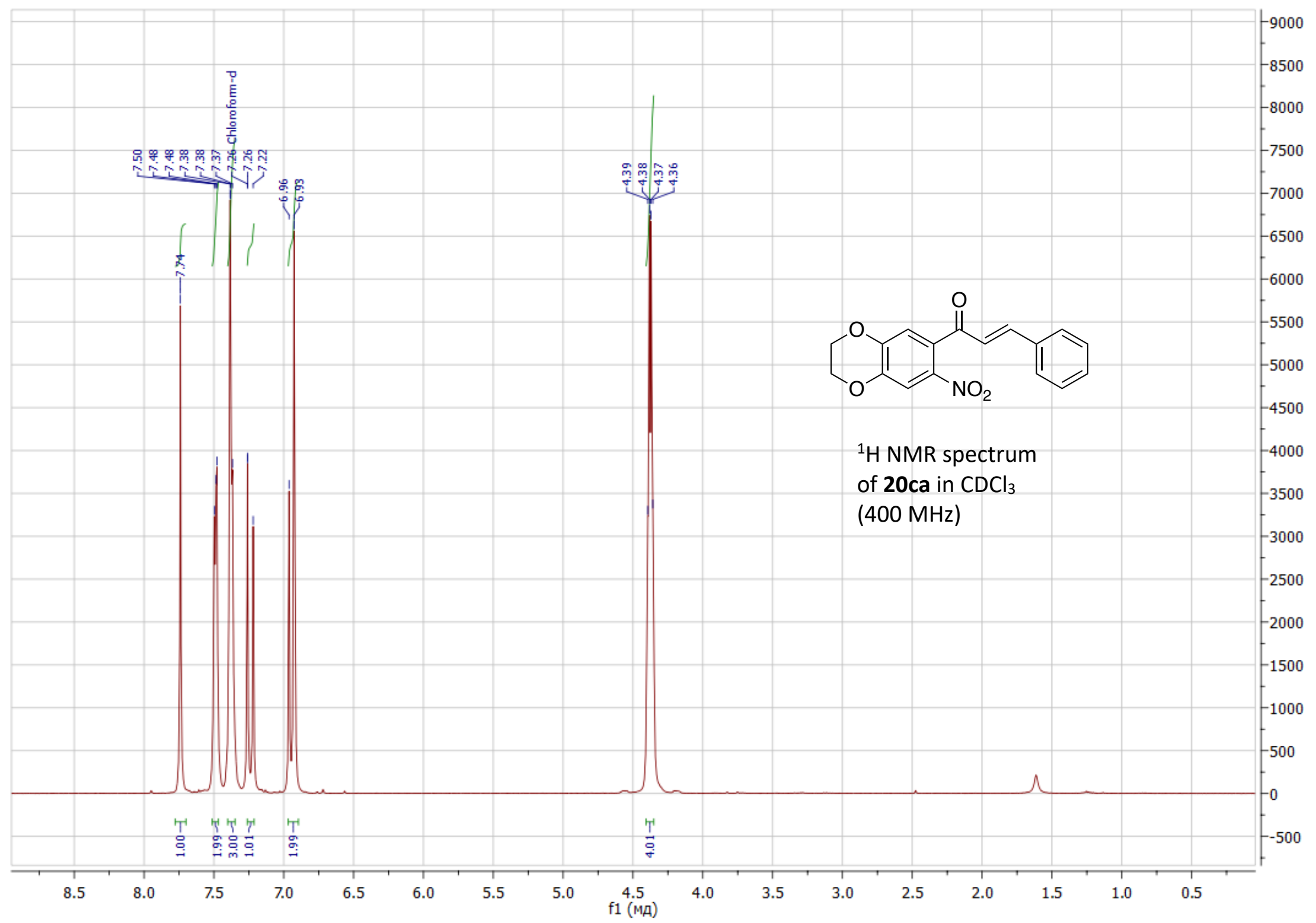




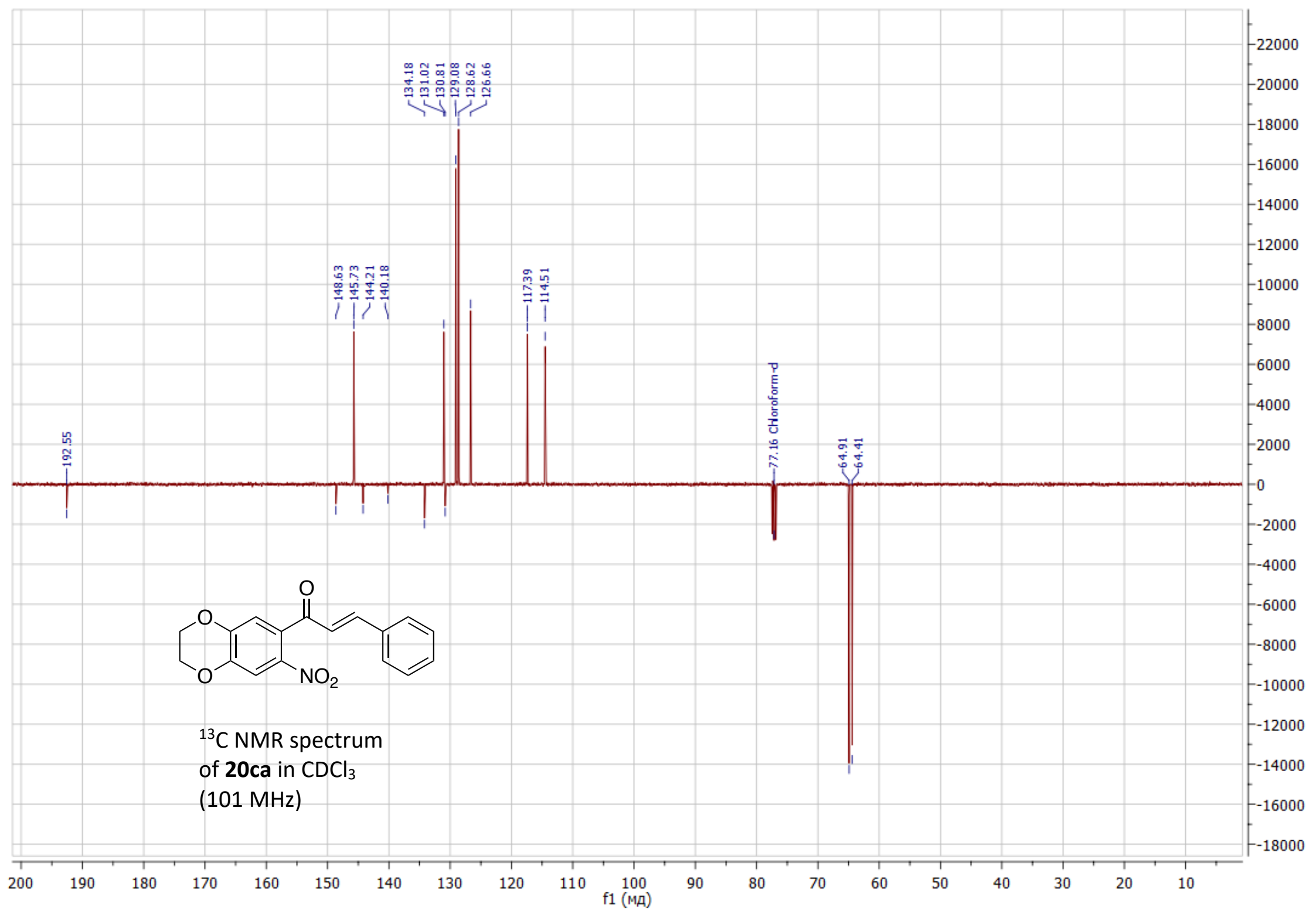




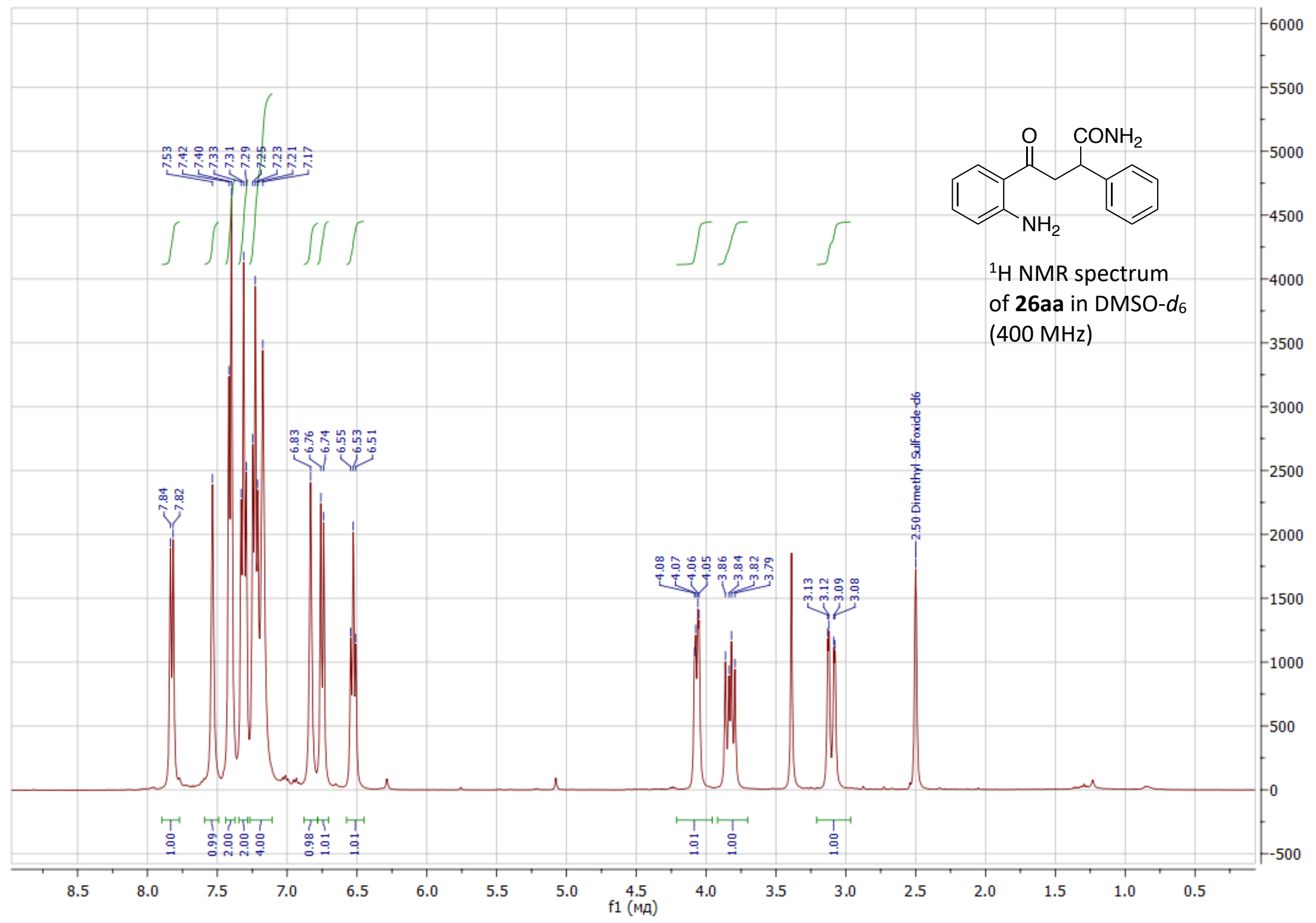




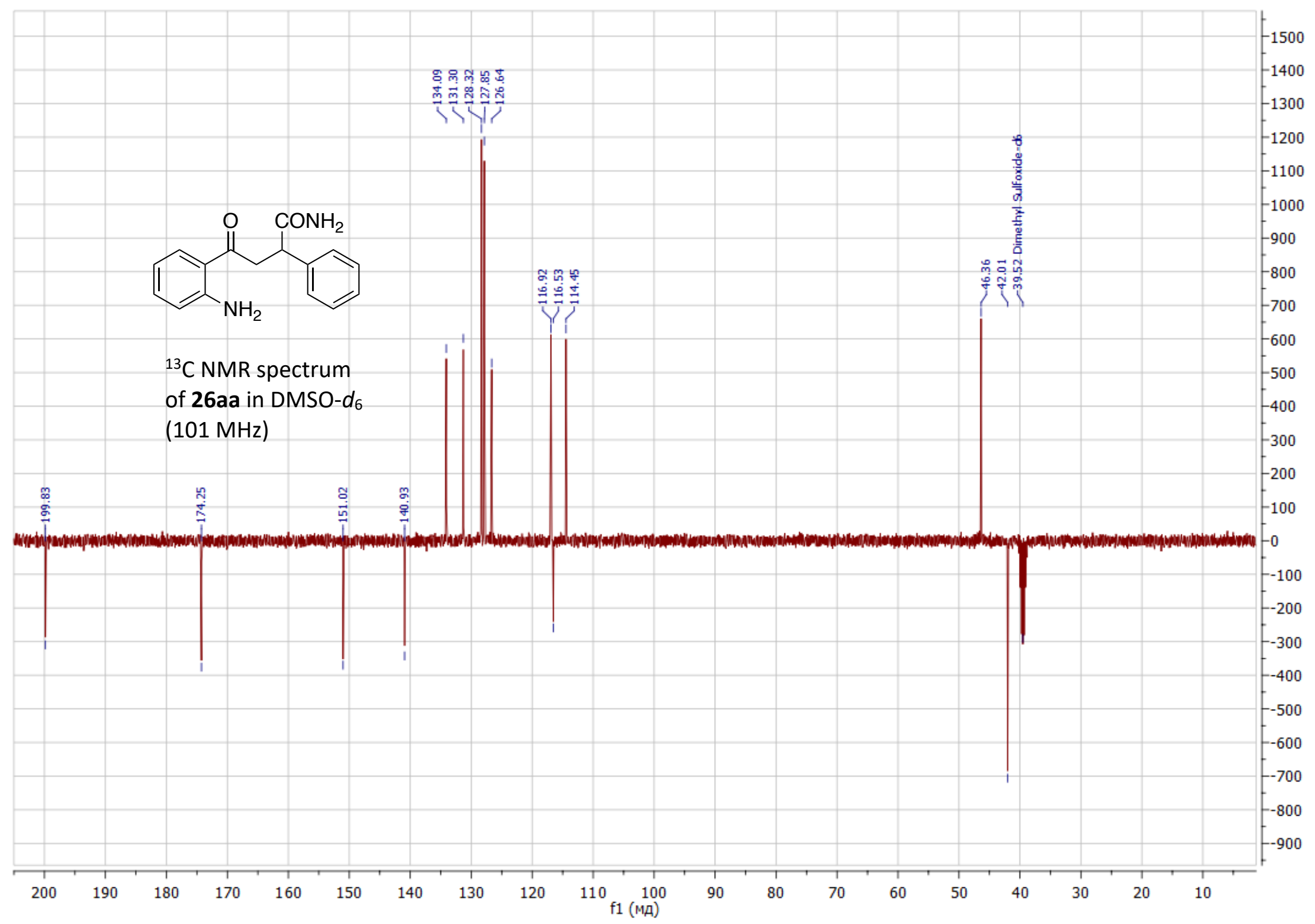




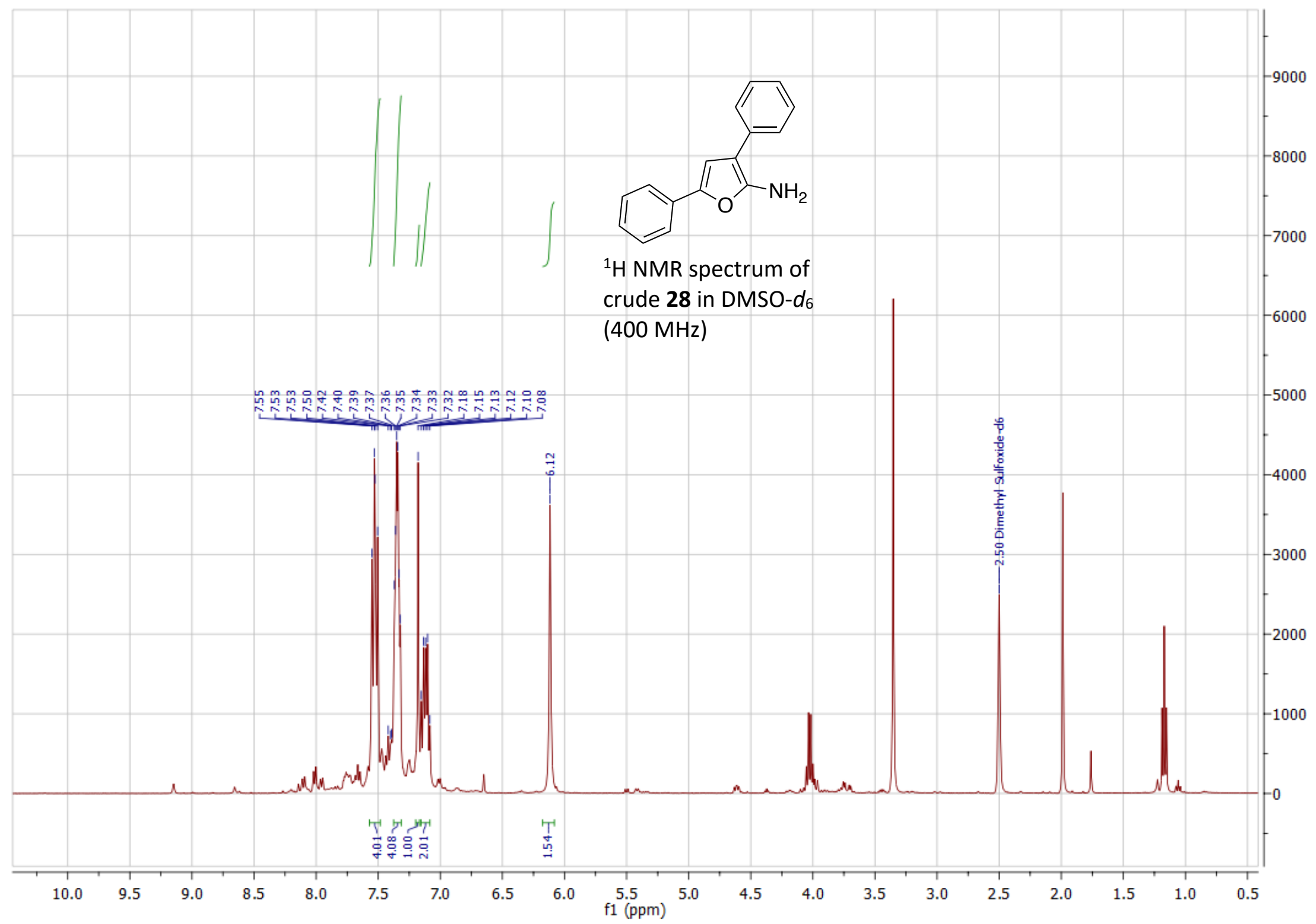




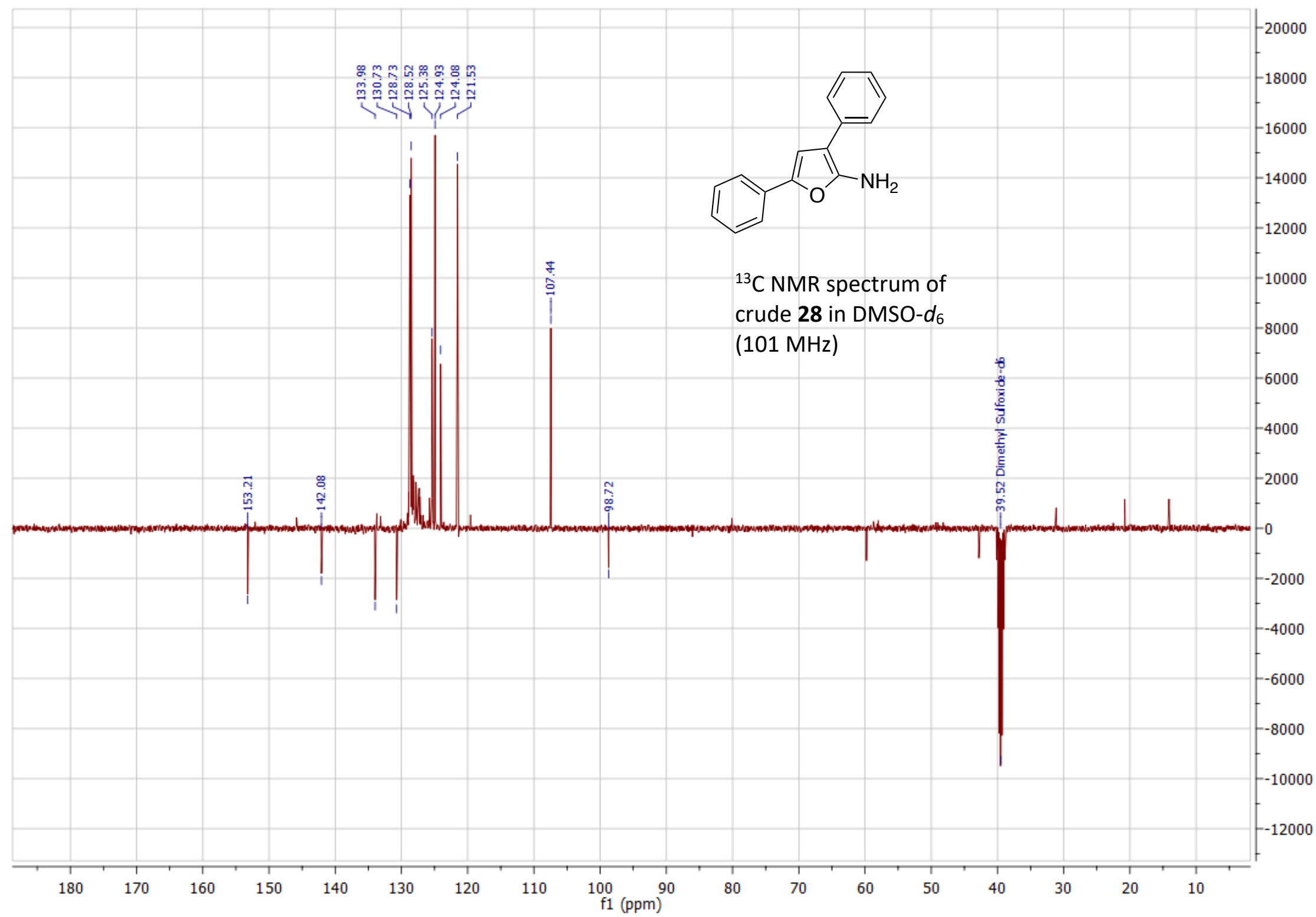




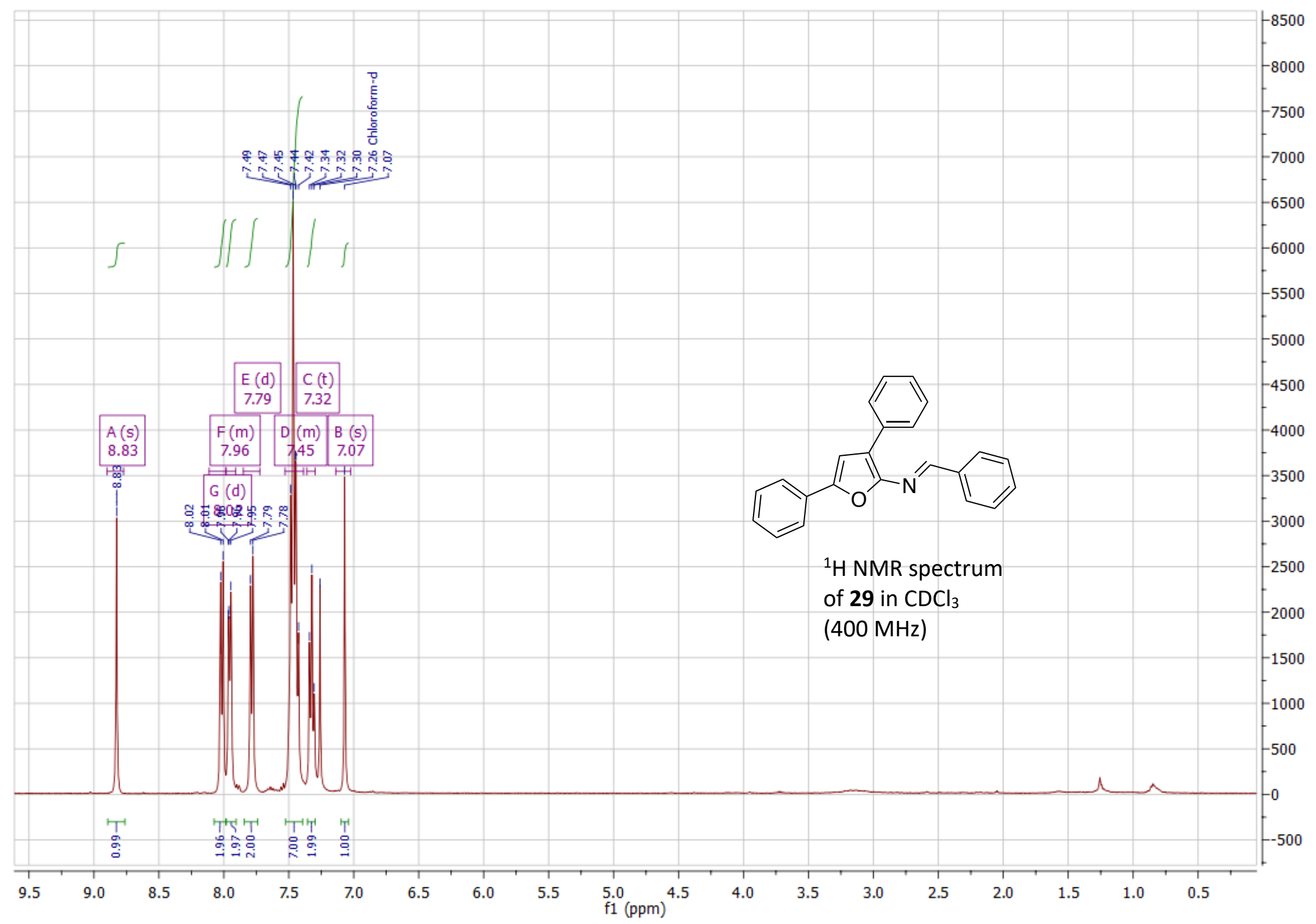




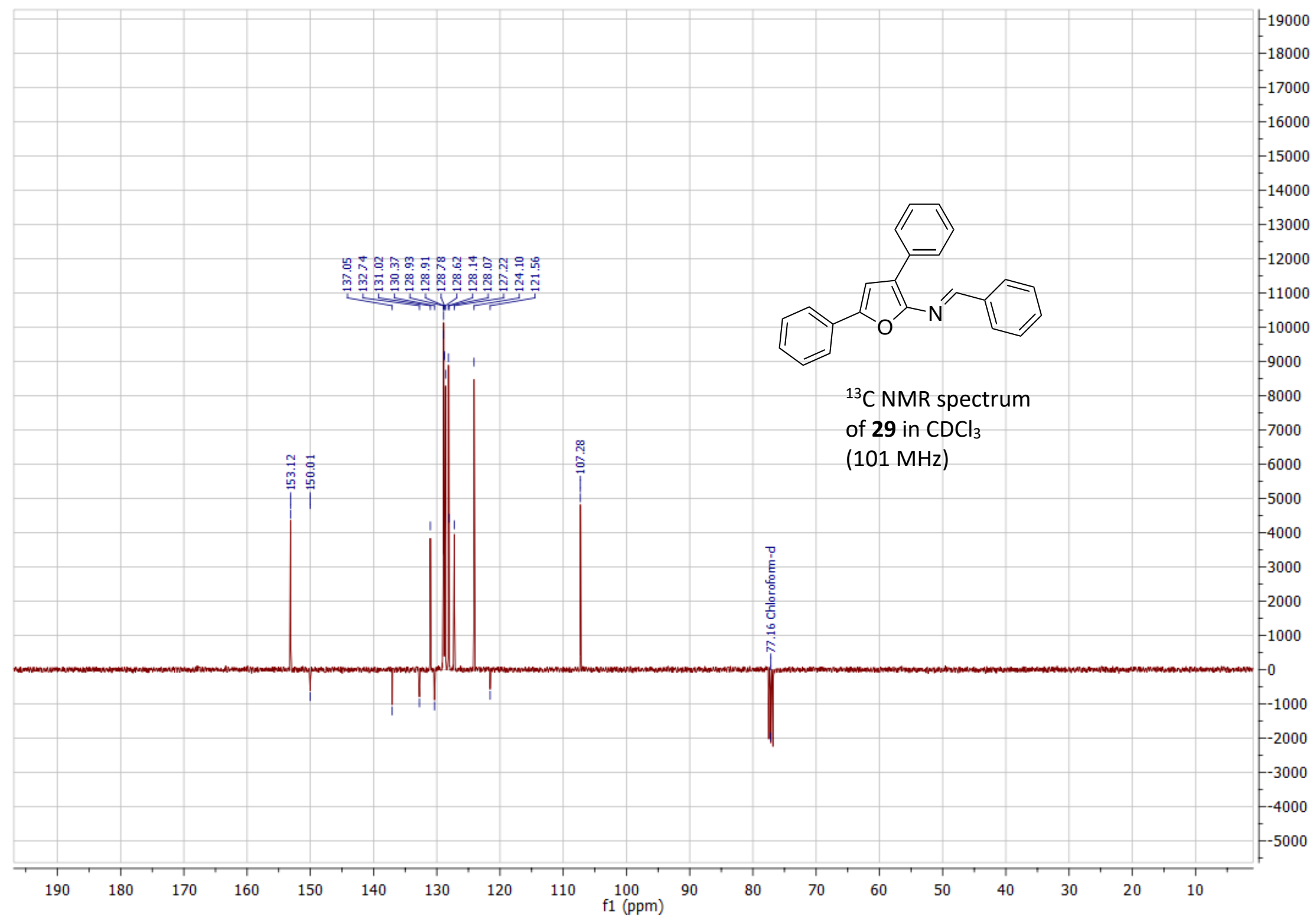




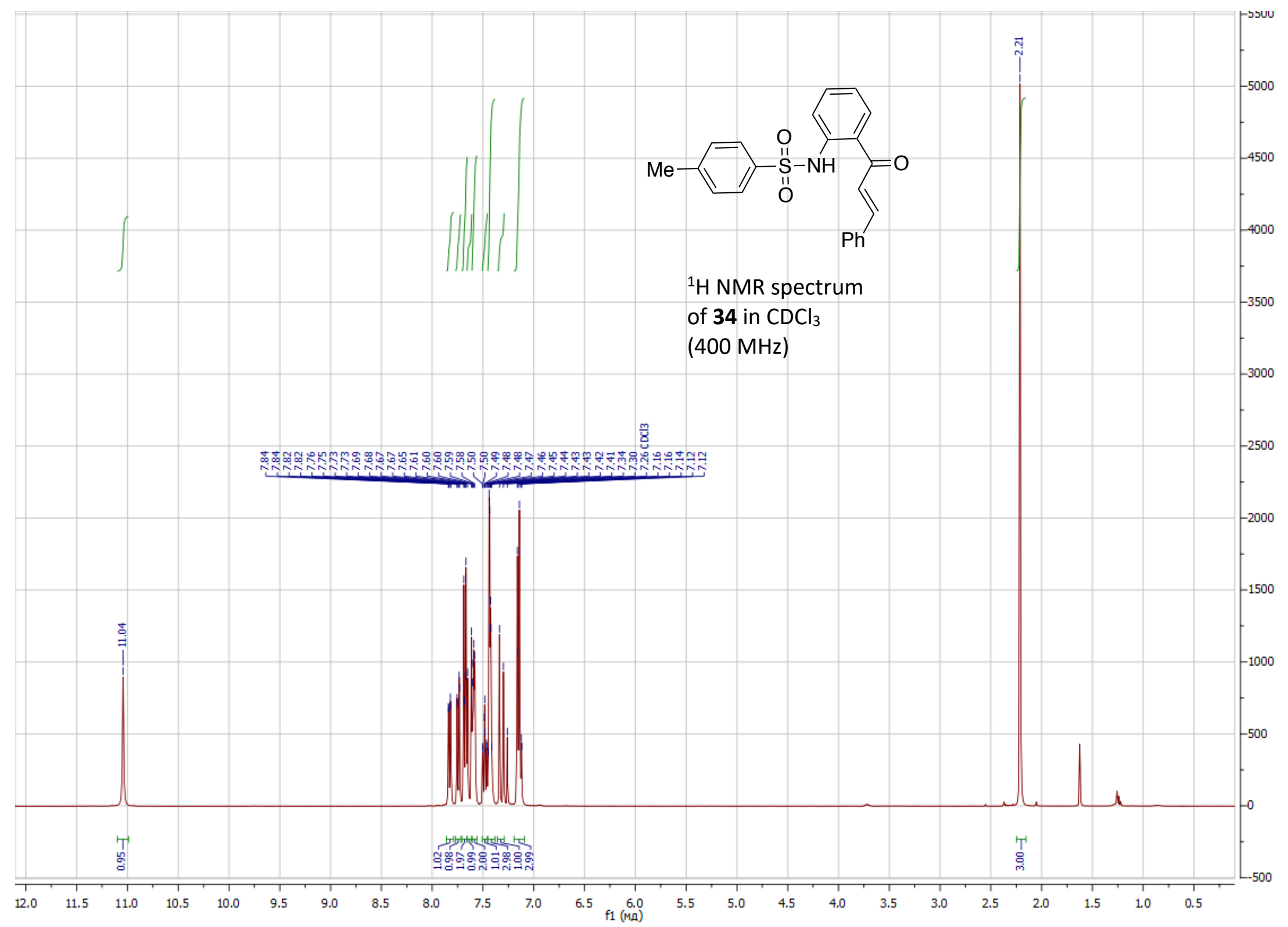




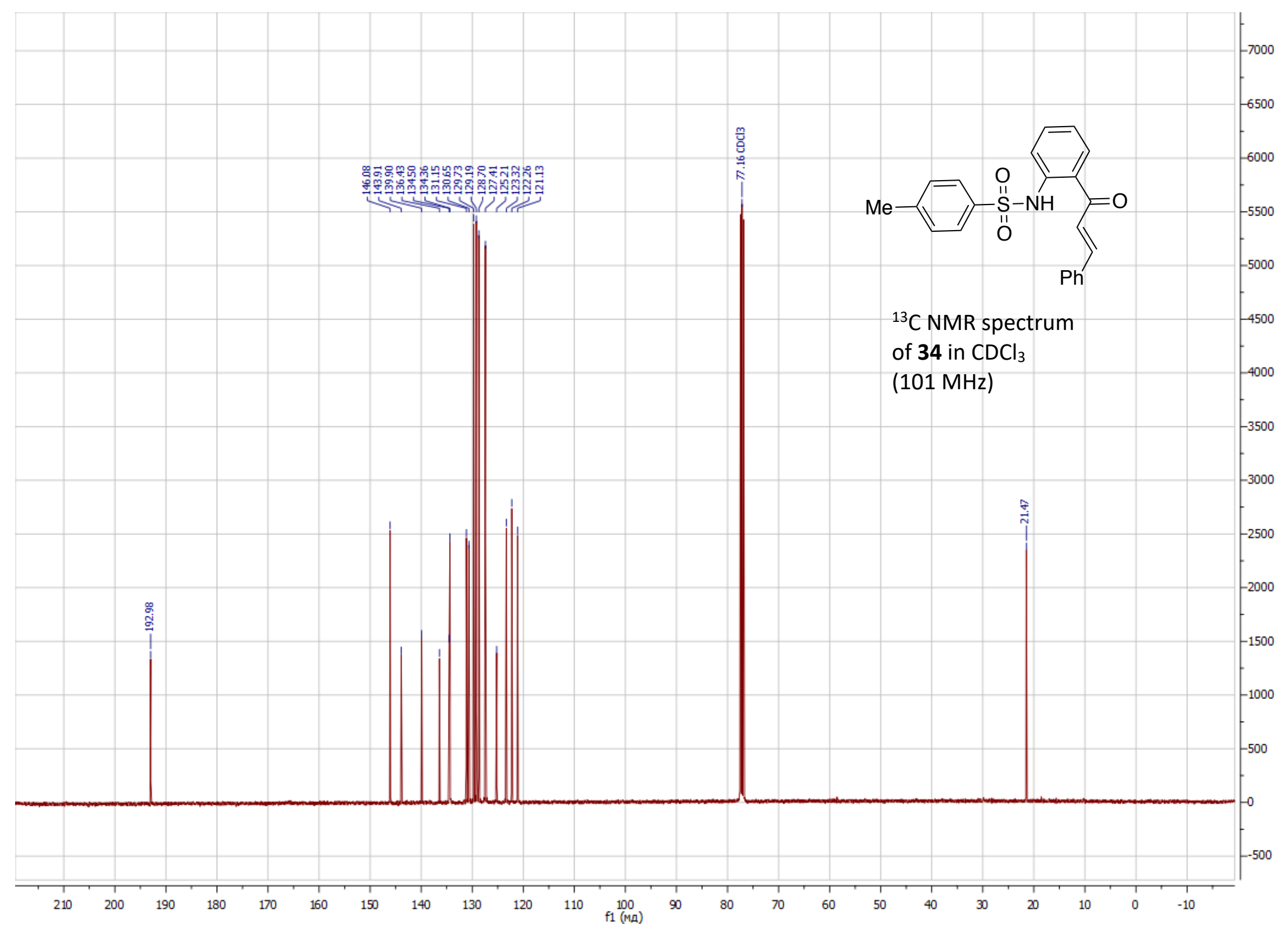




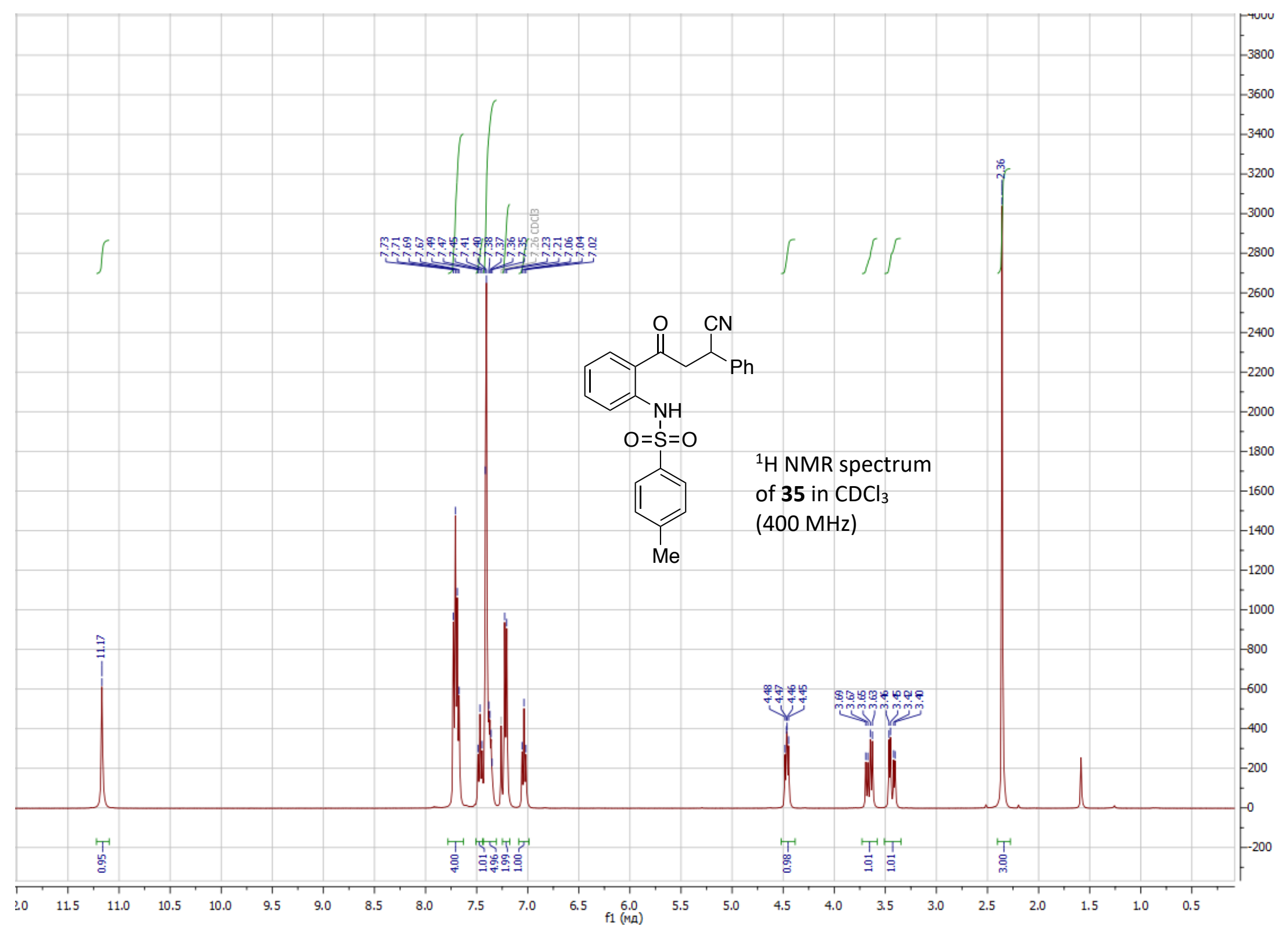




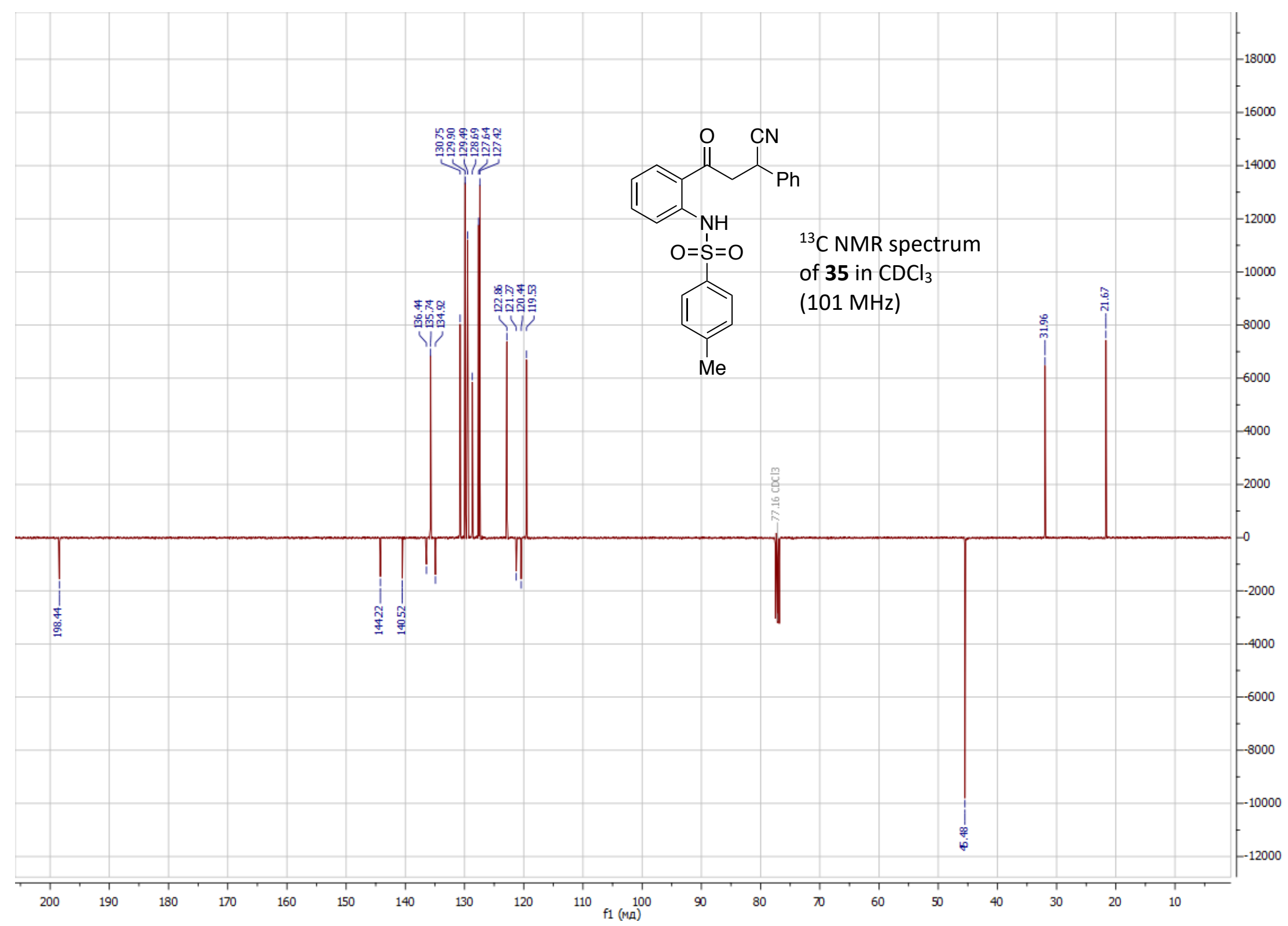




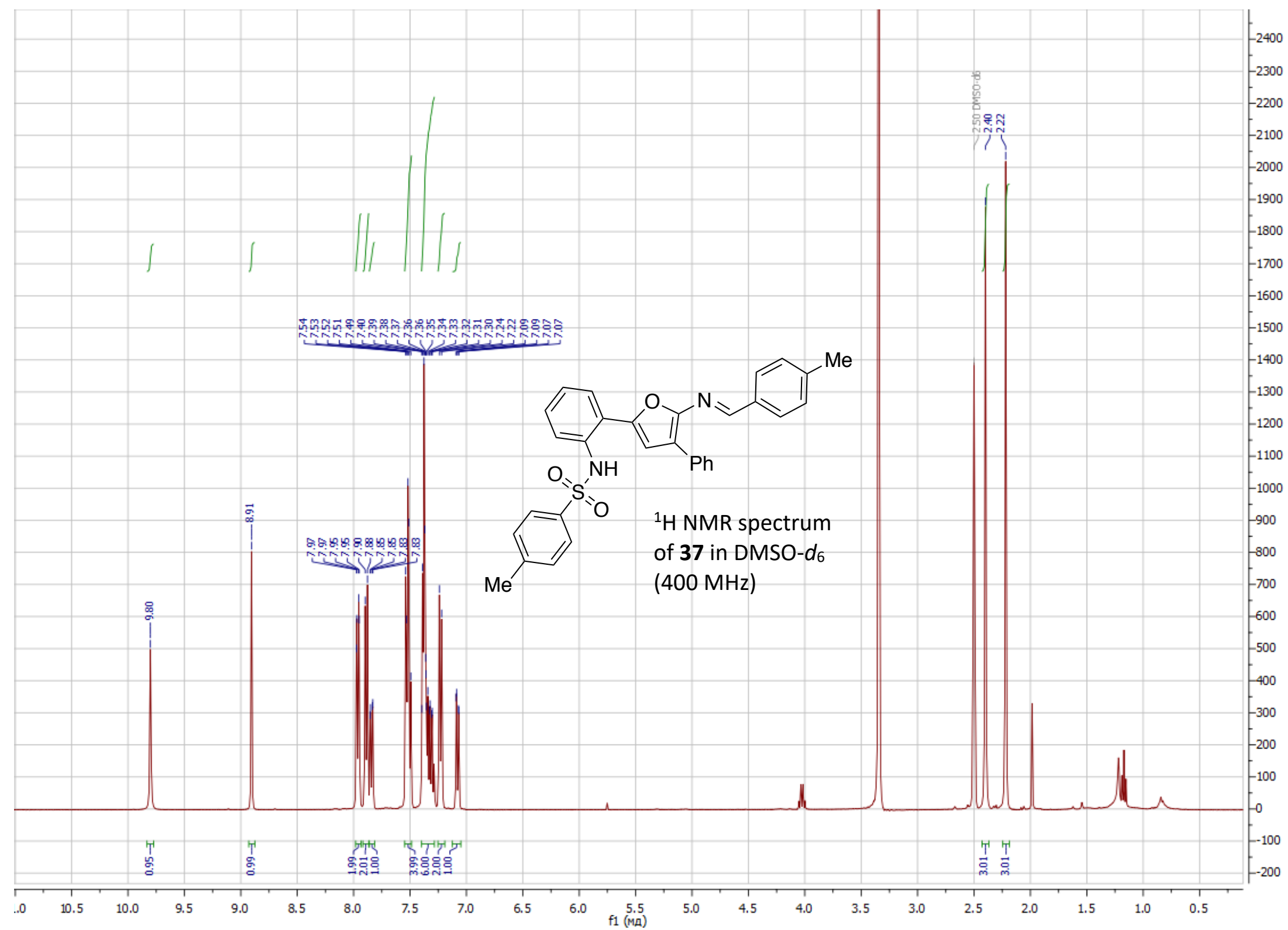




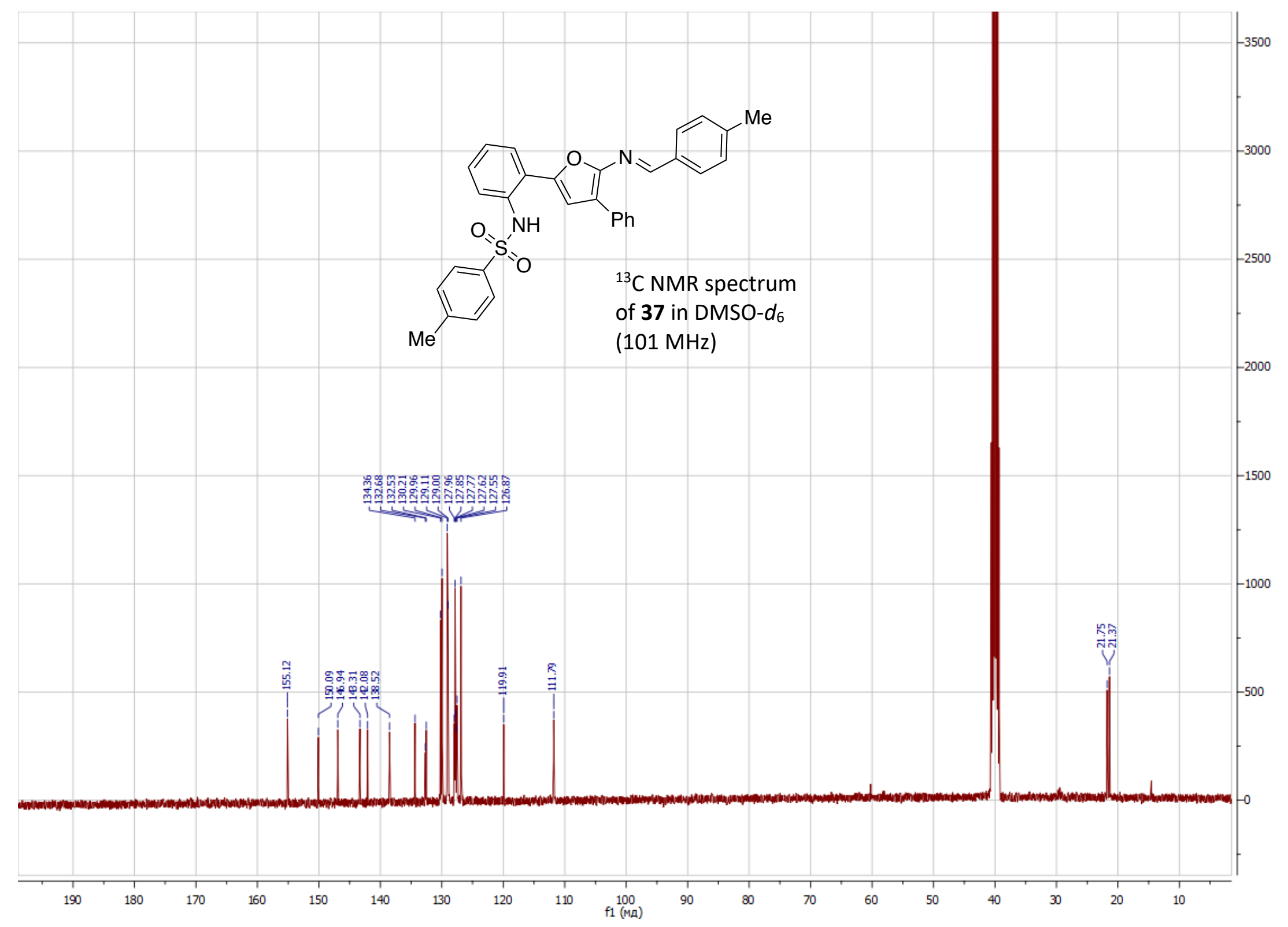




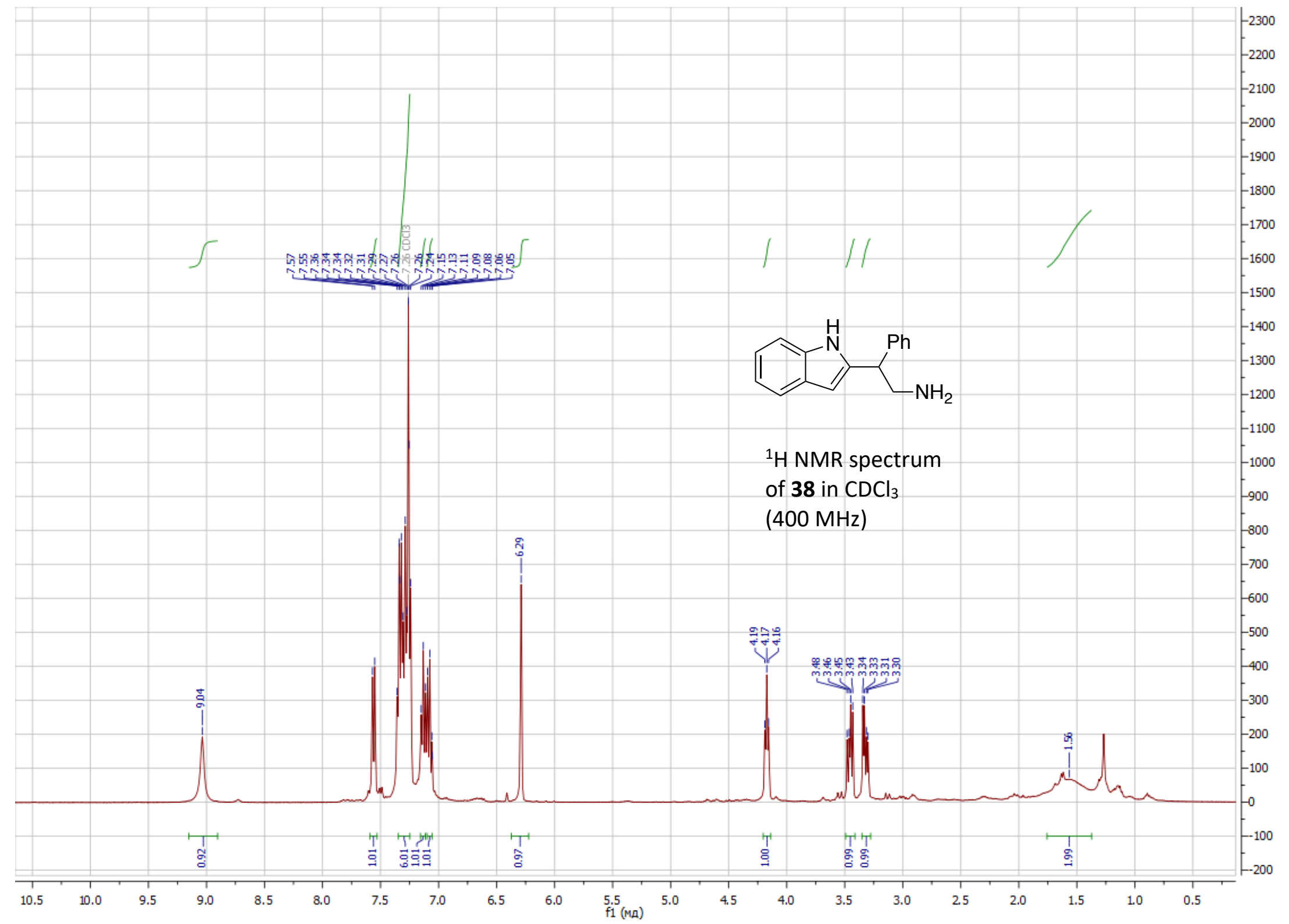




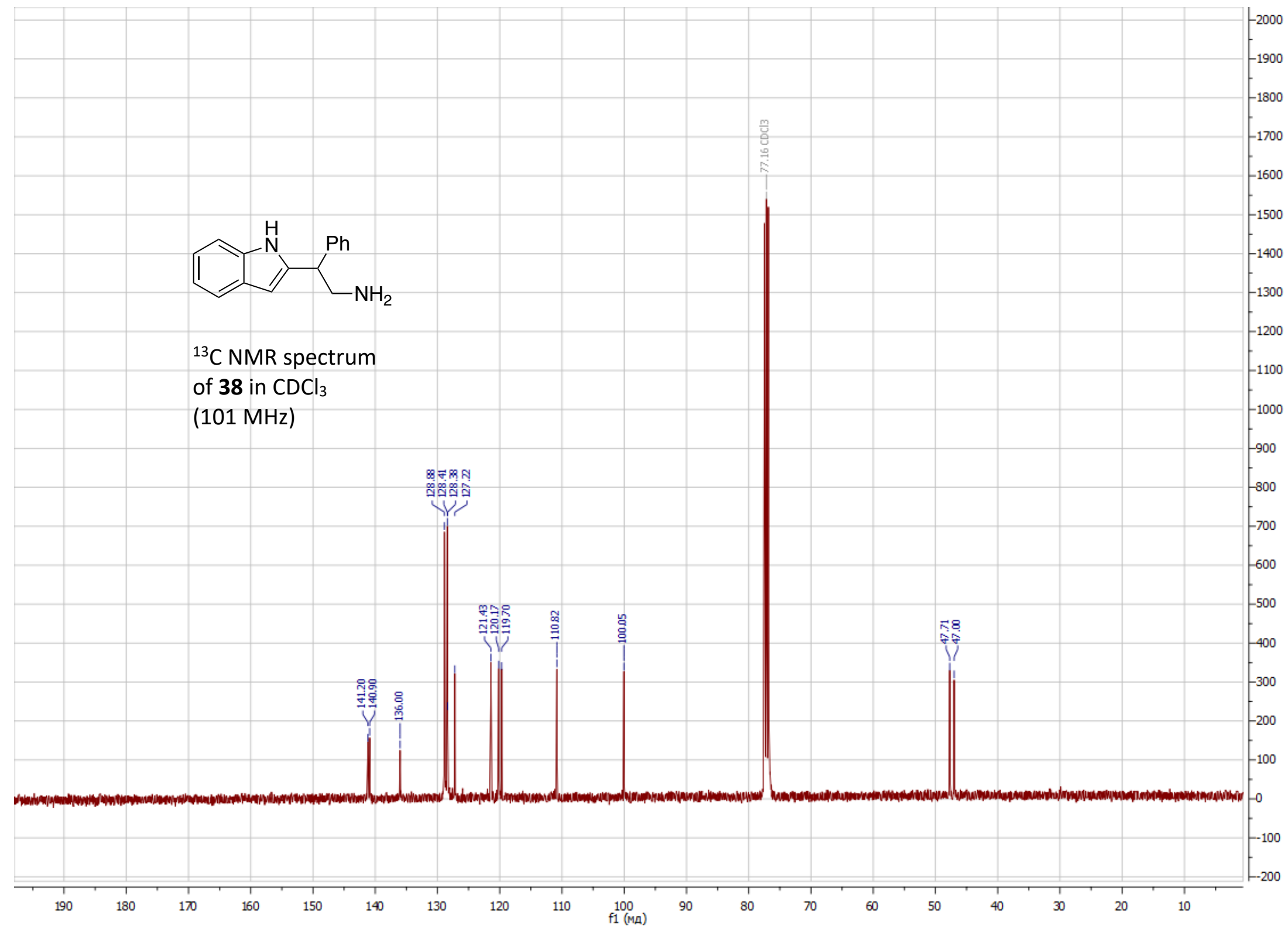




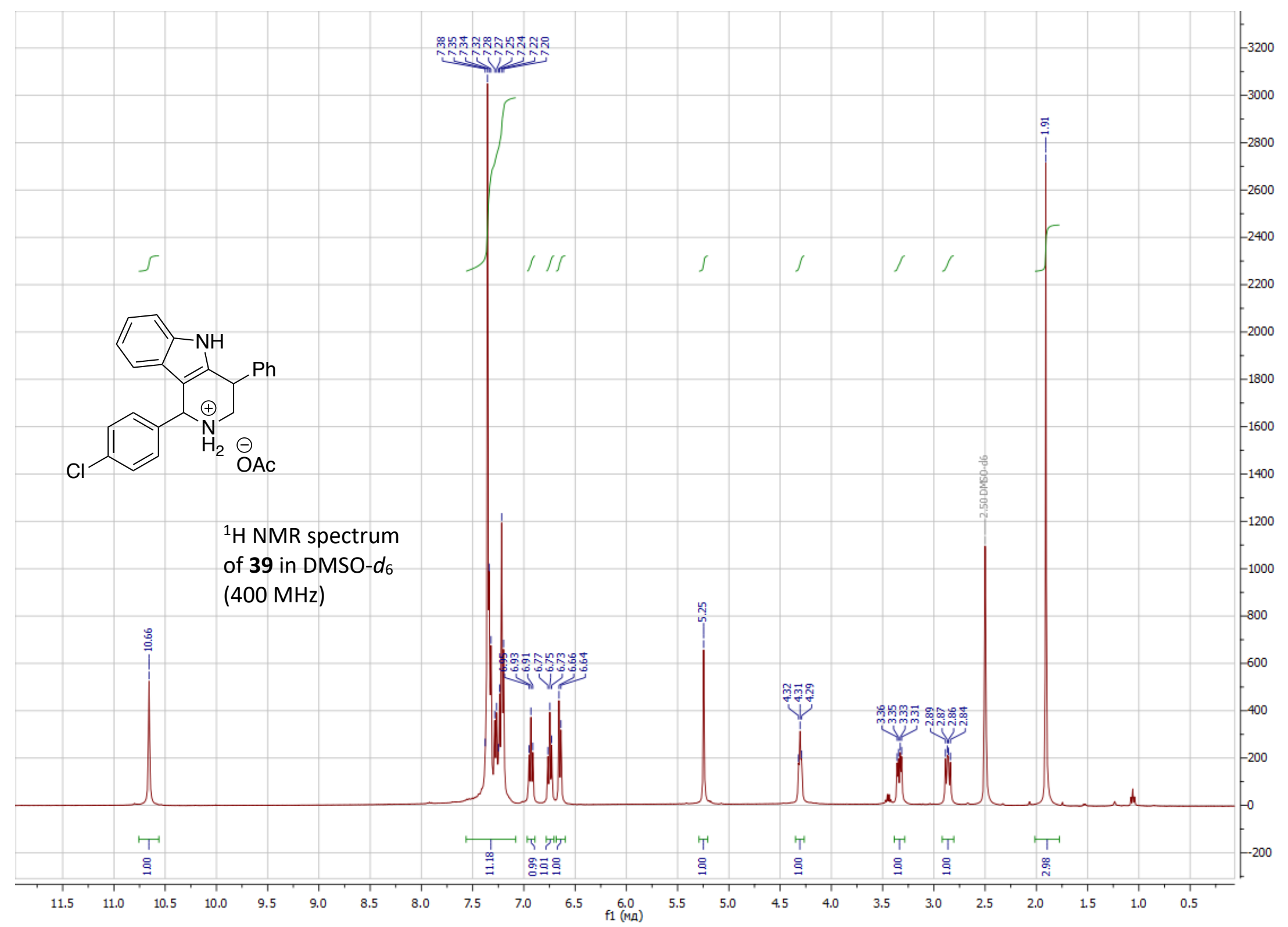




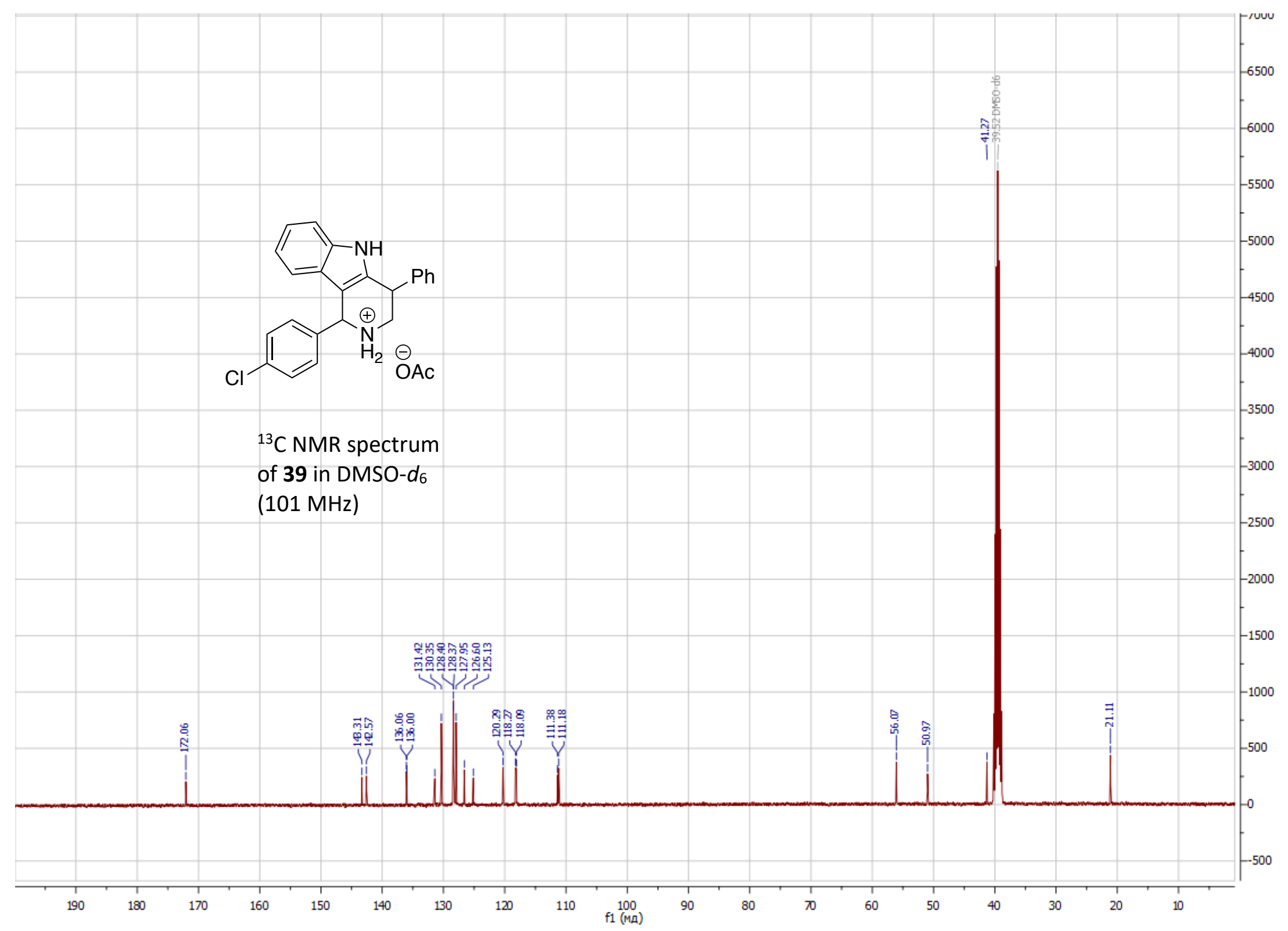




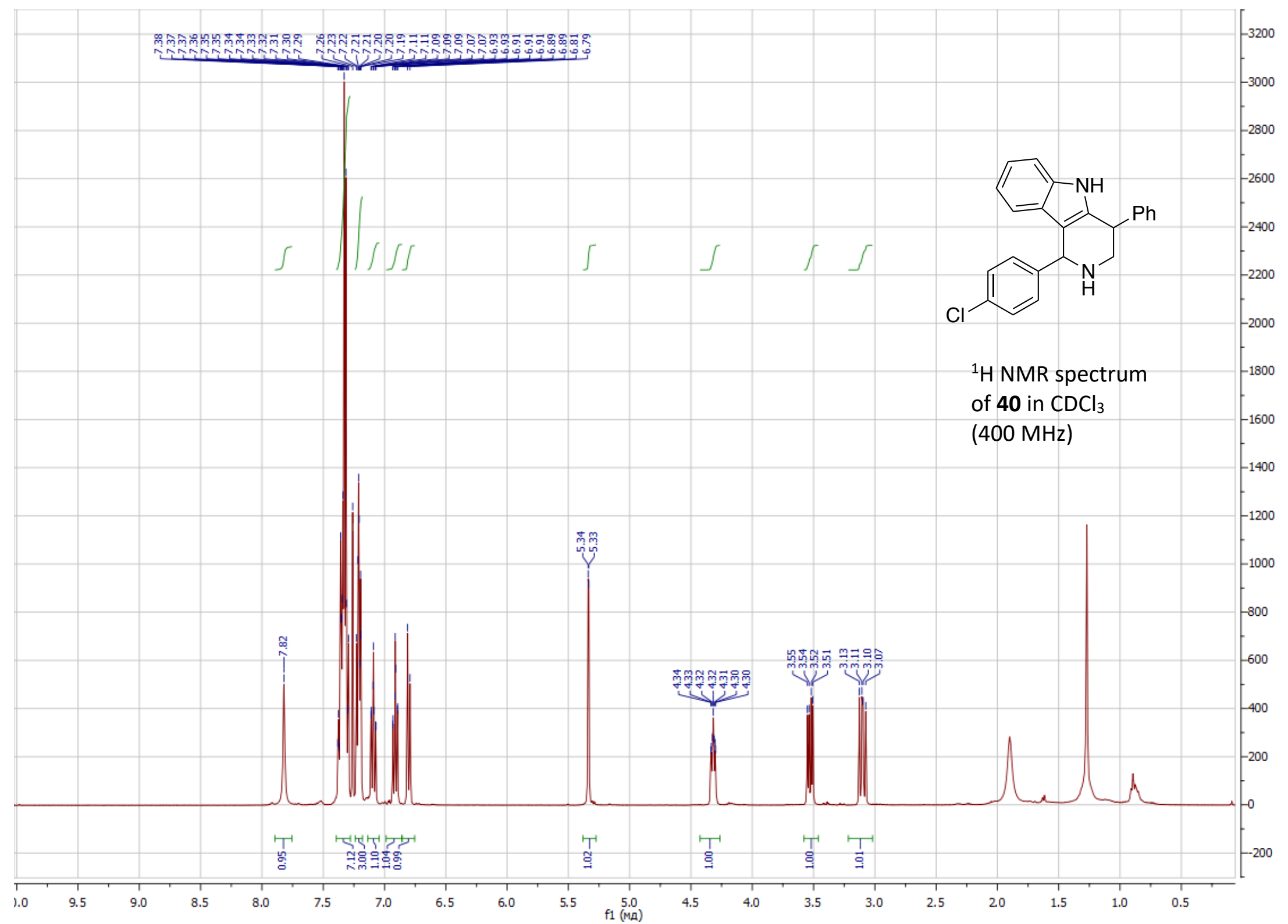




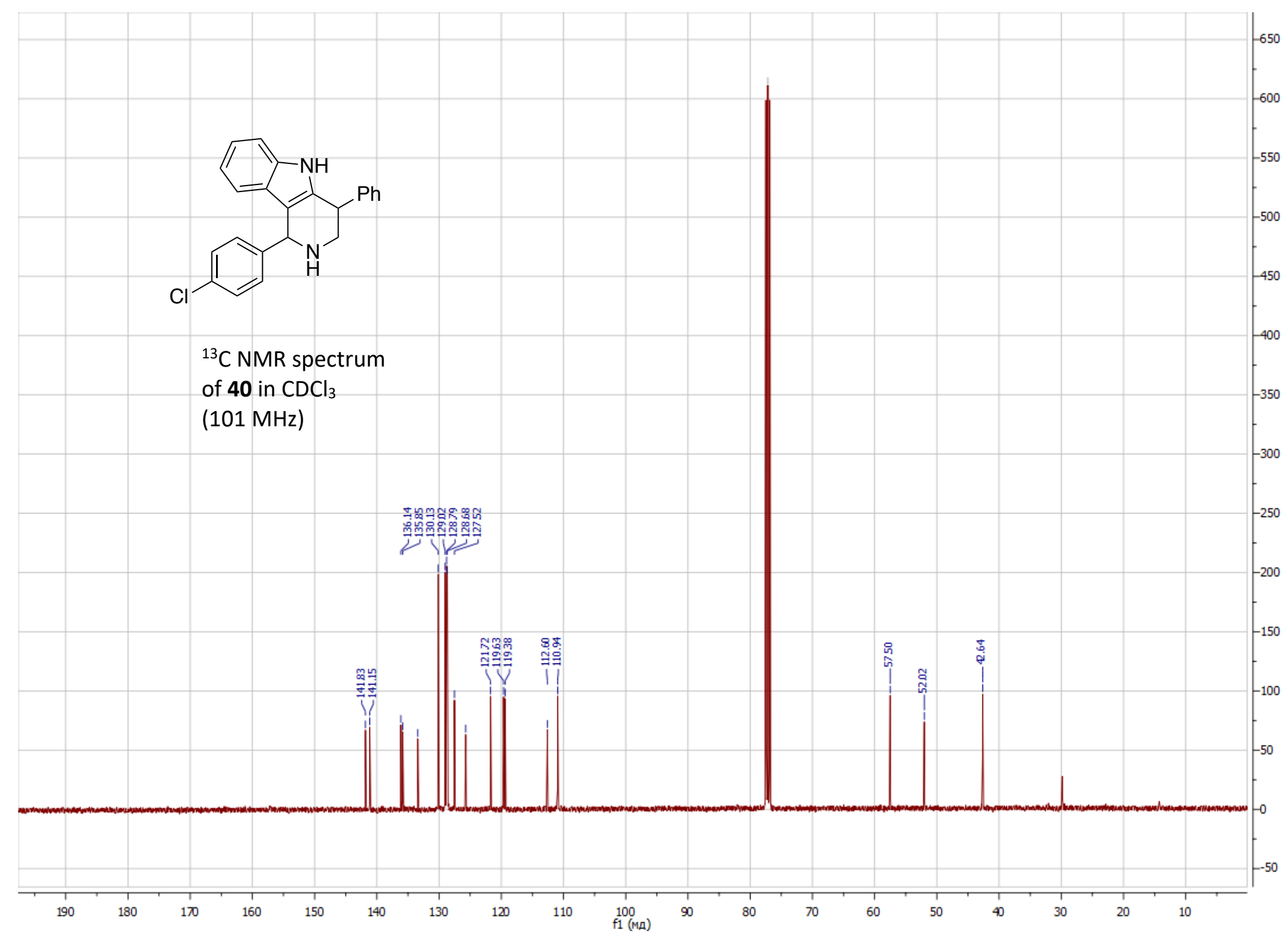




\section{HRMS Spectral Charts of 2-(1H-Indol-2-yl)acetamides 1}

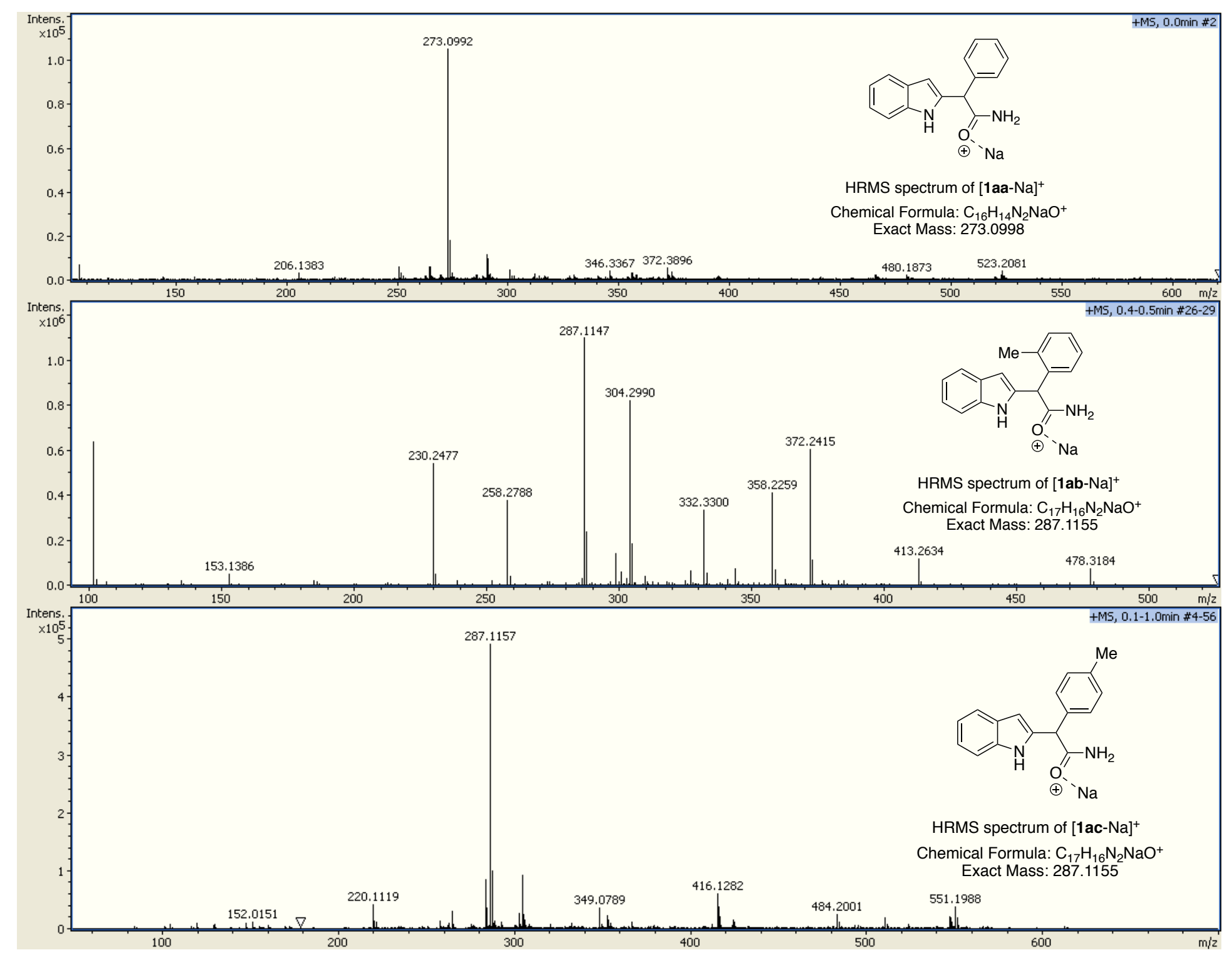




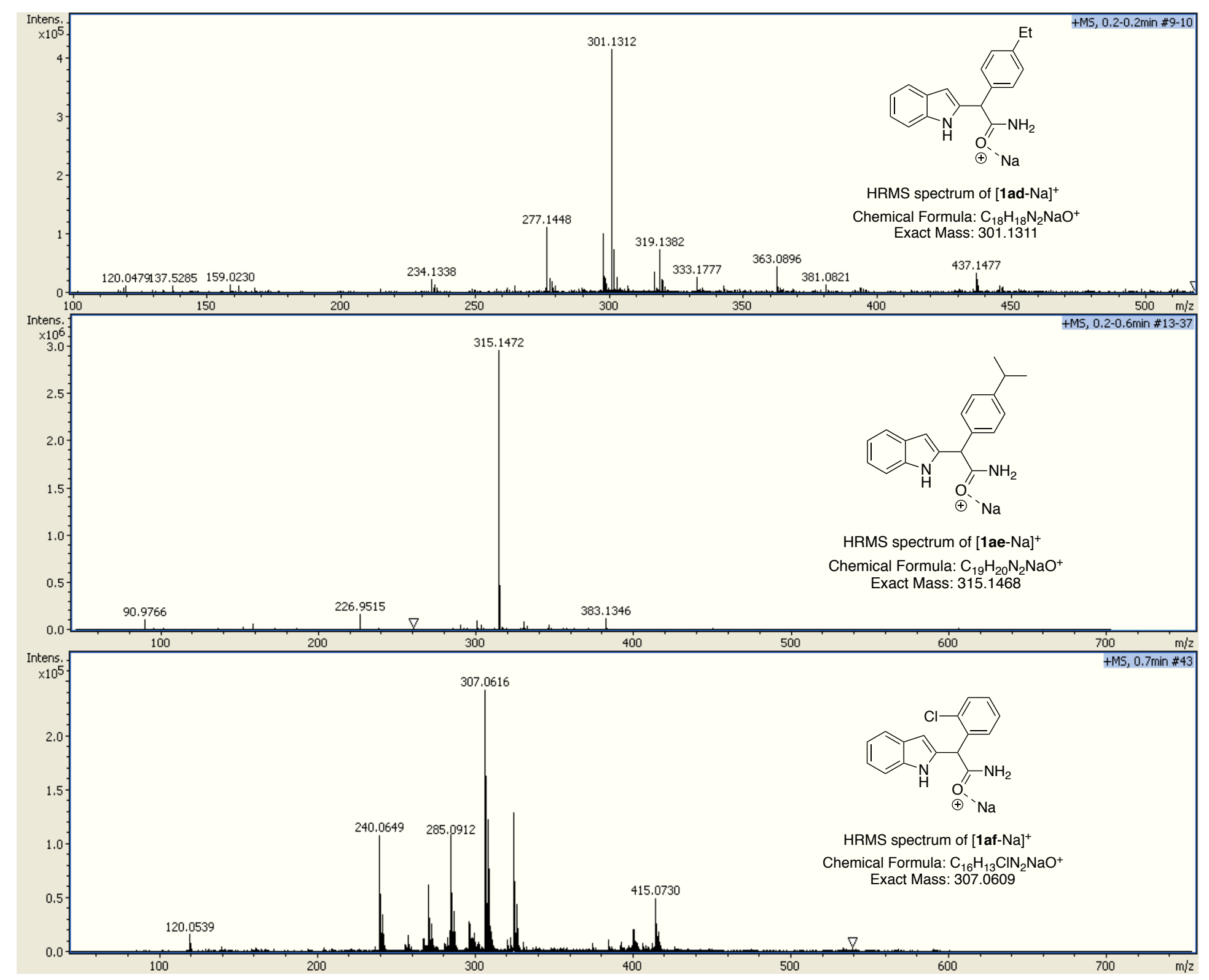




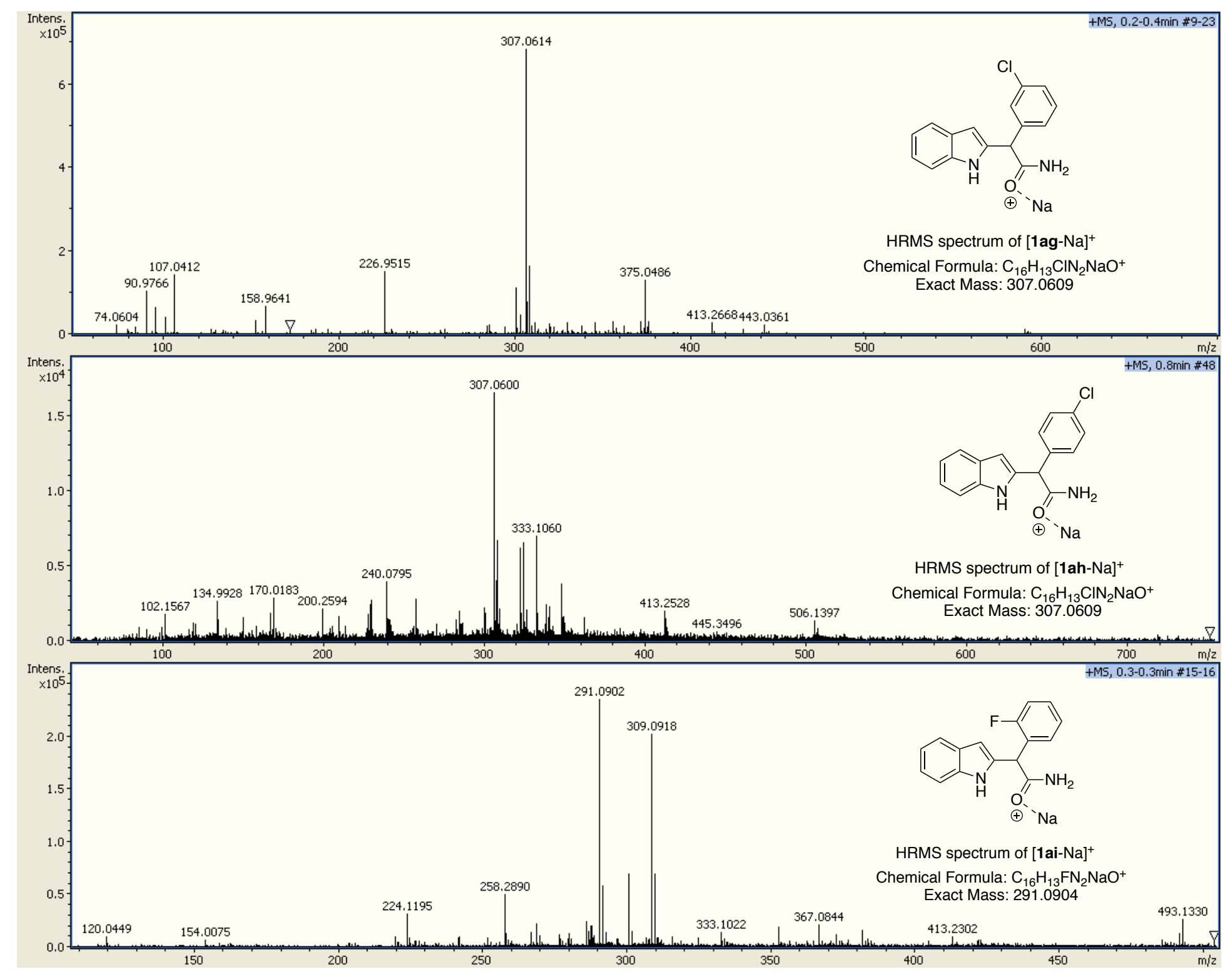




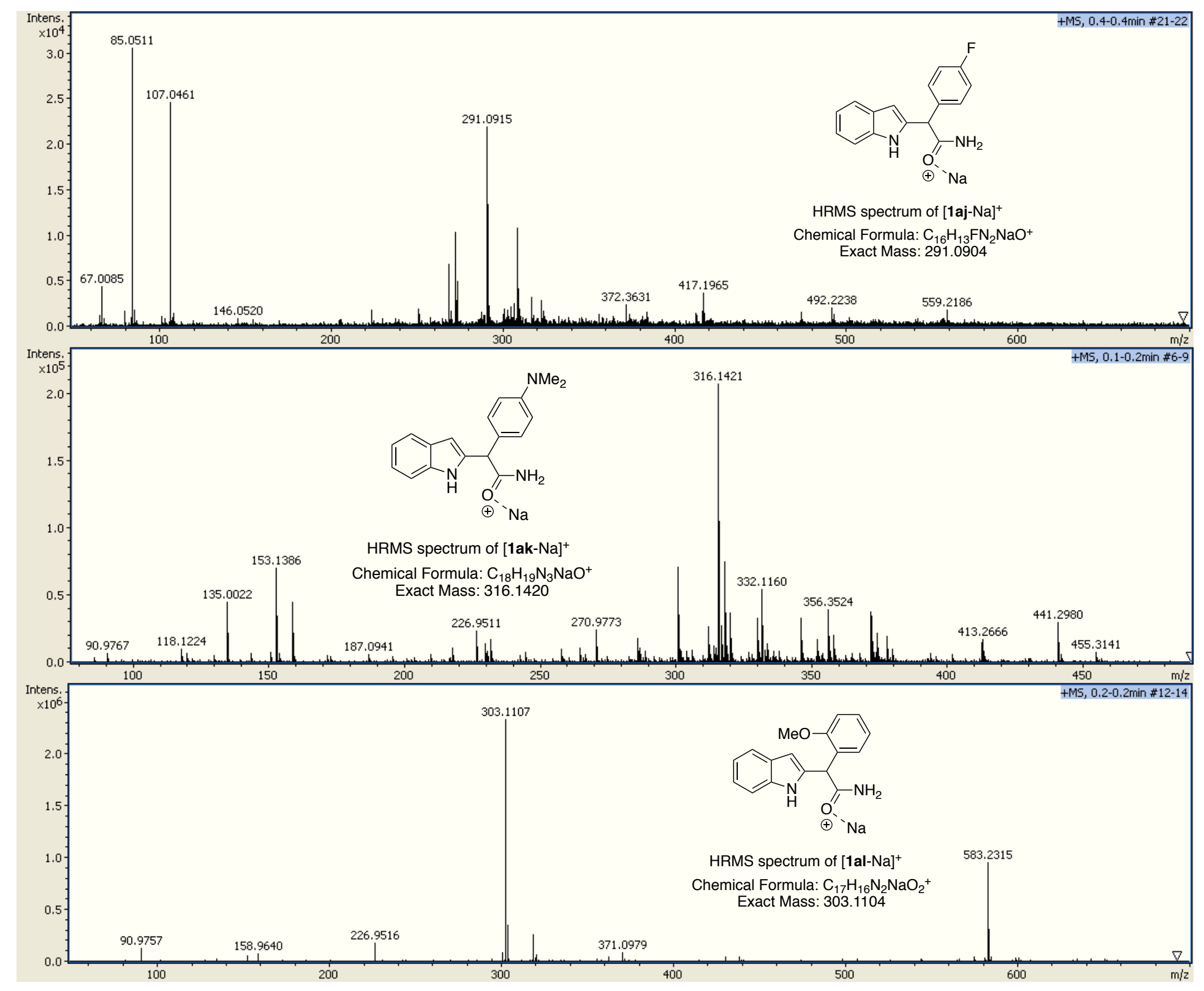




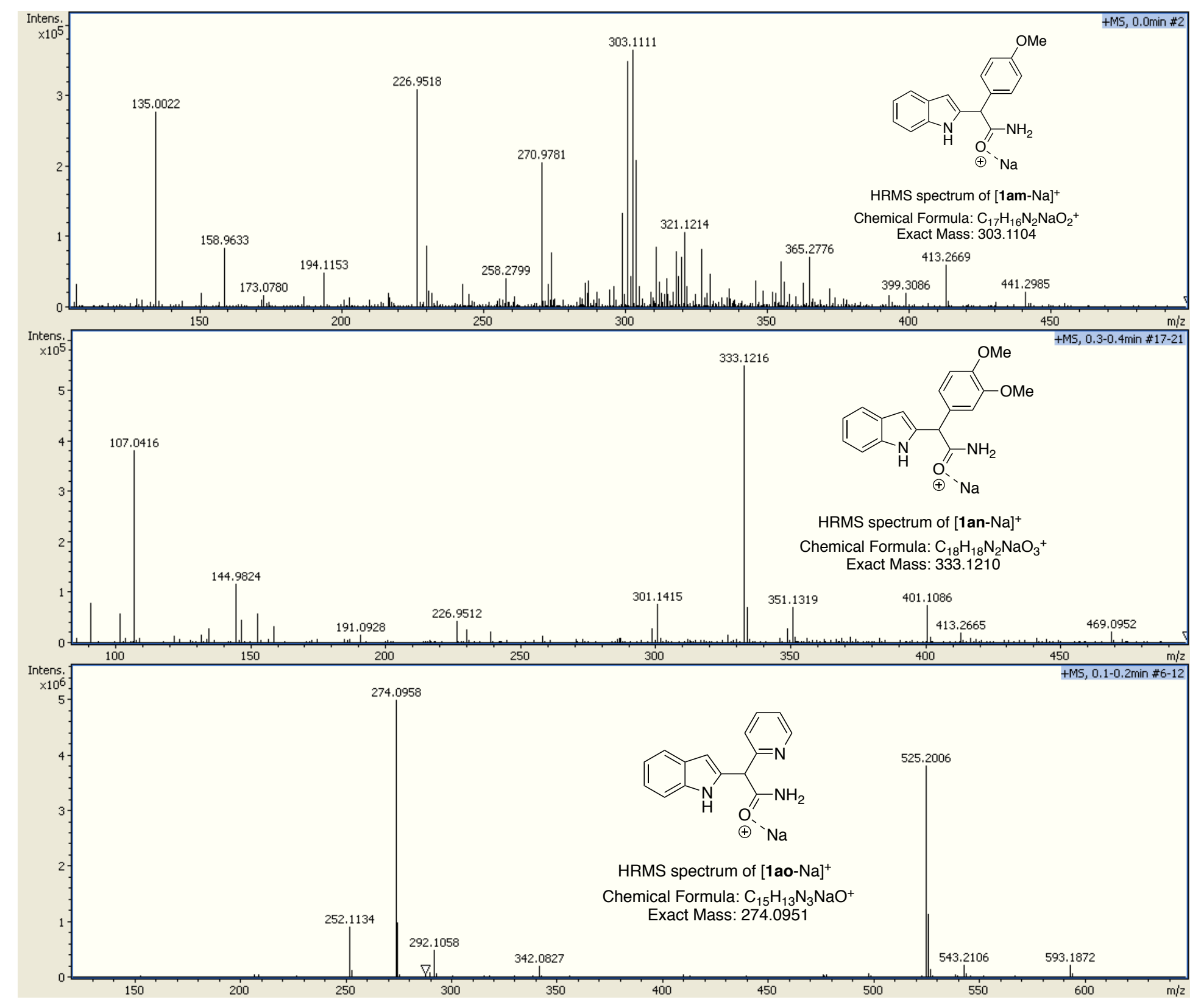




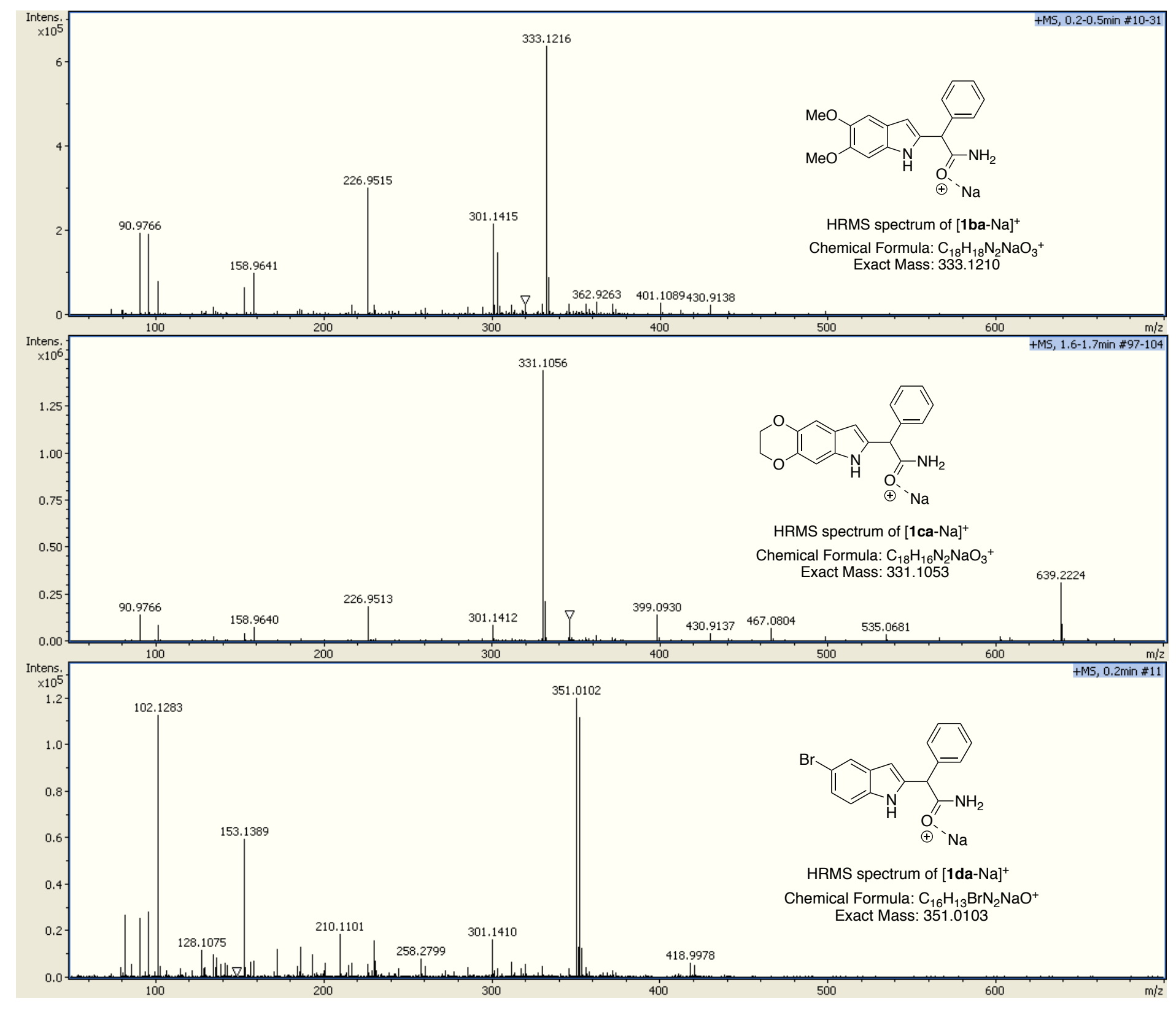




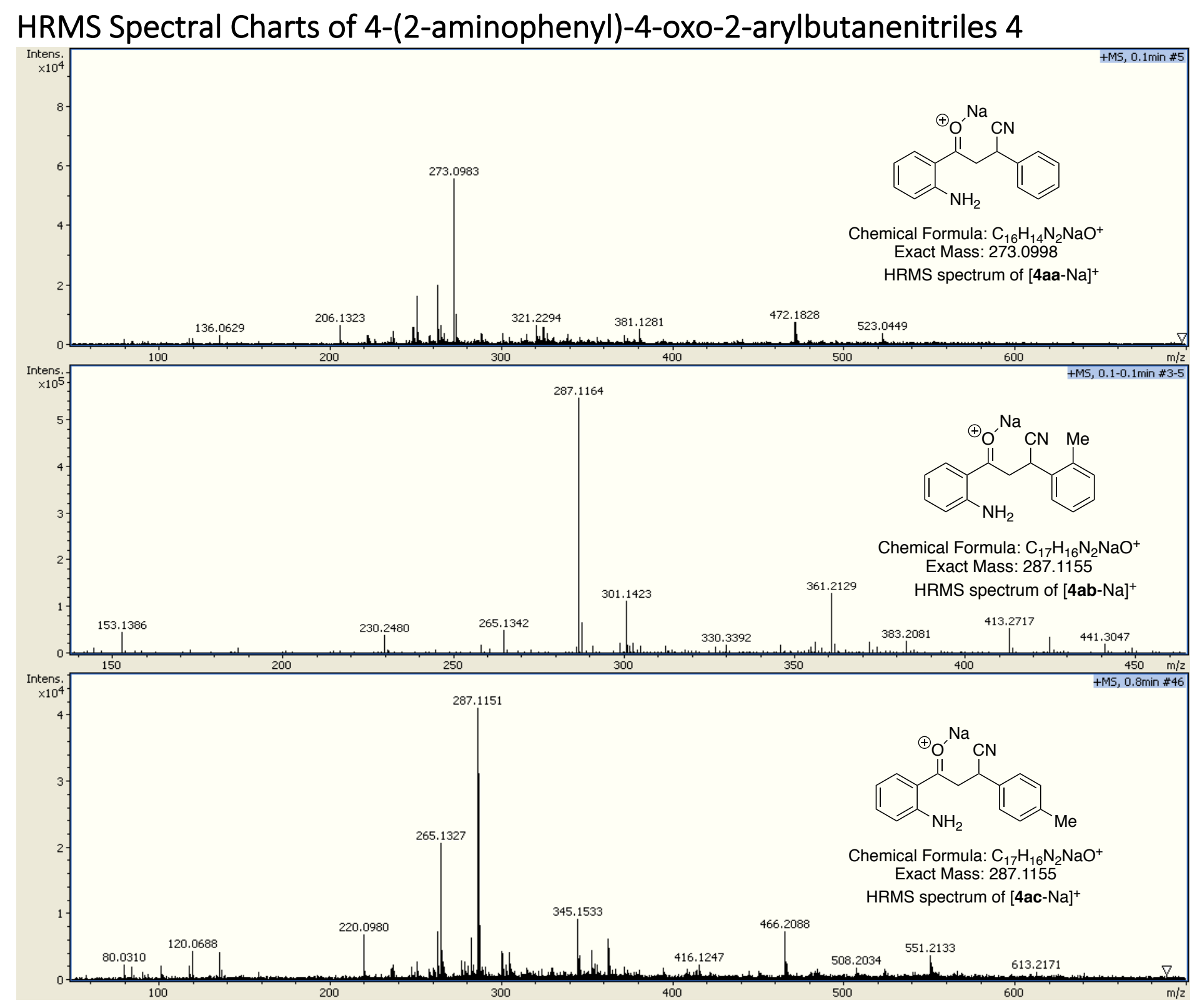




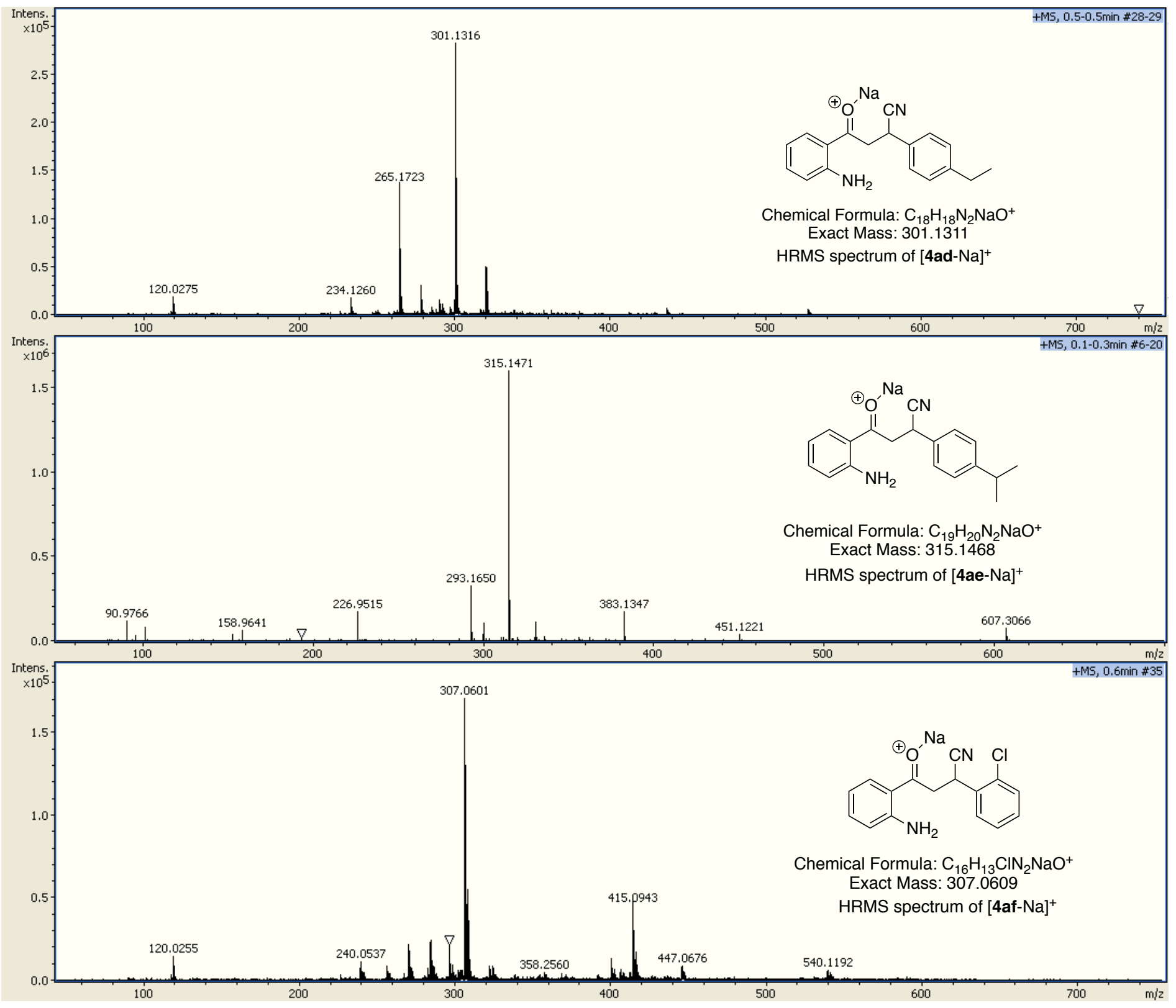

S173 


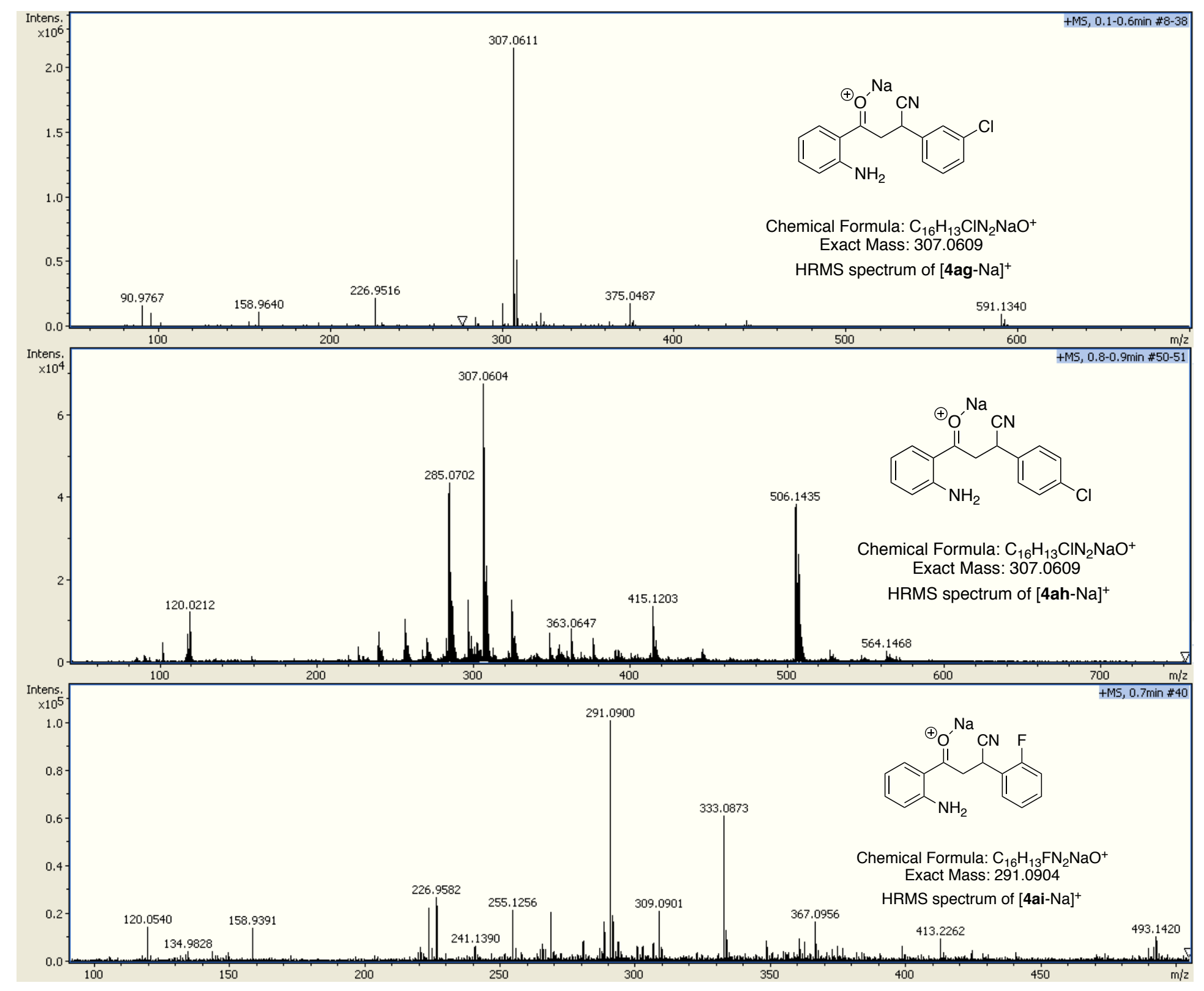




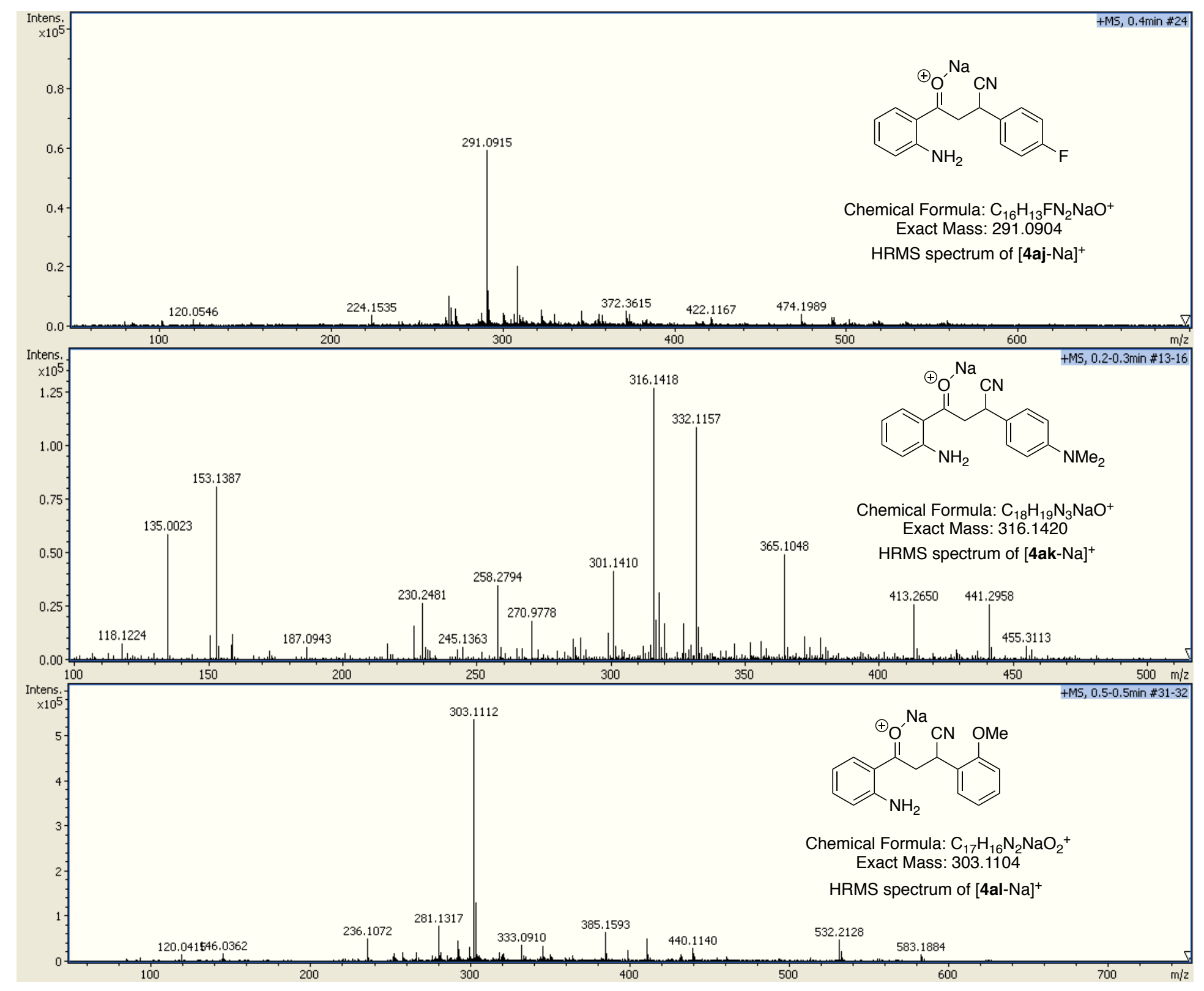




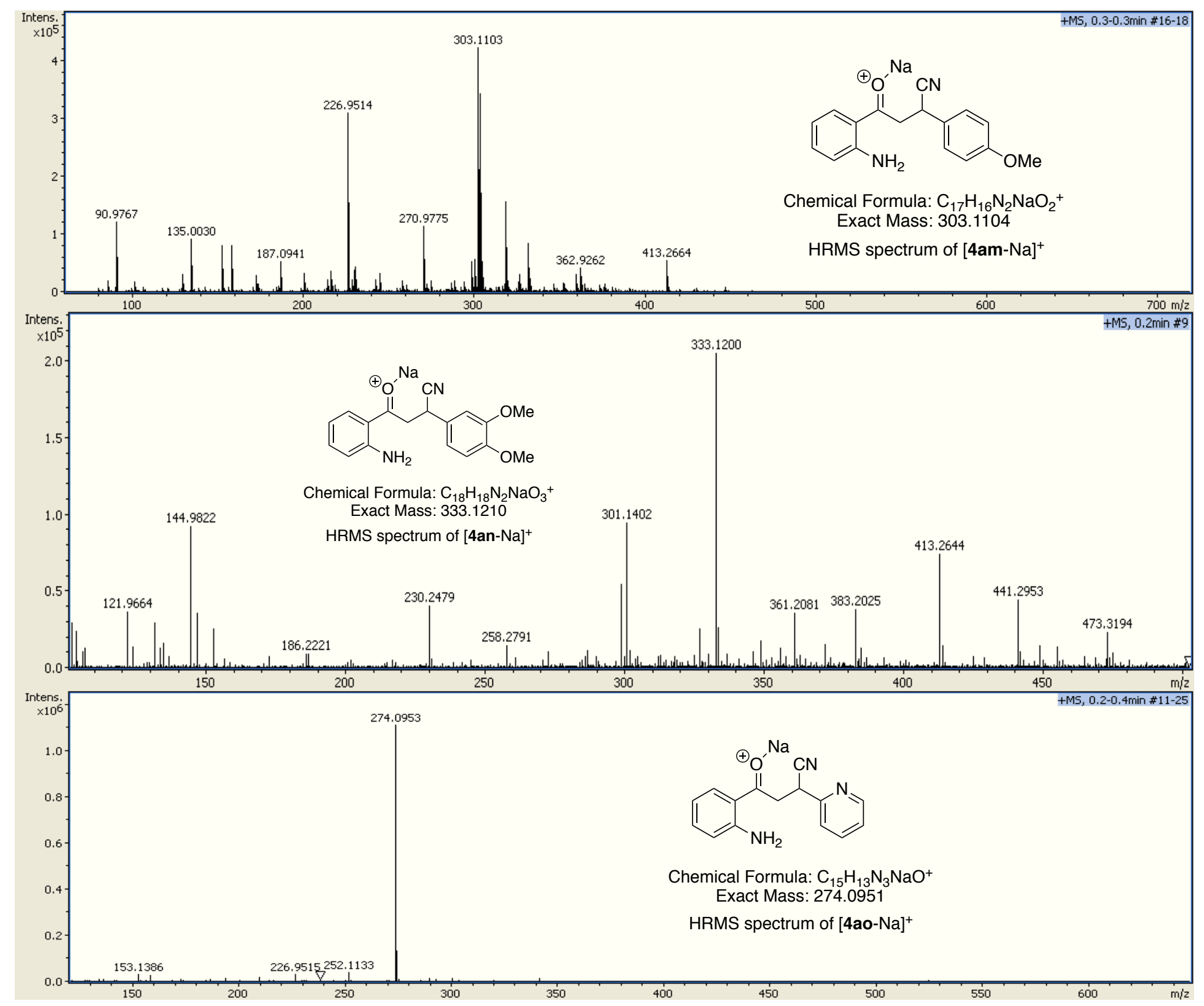




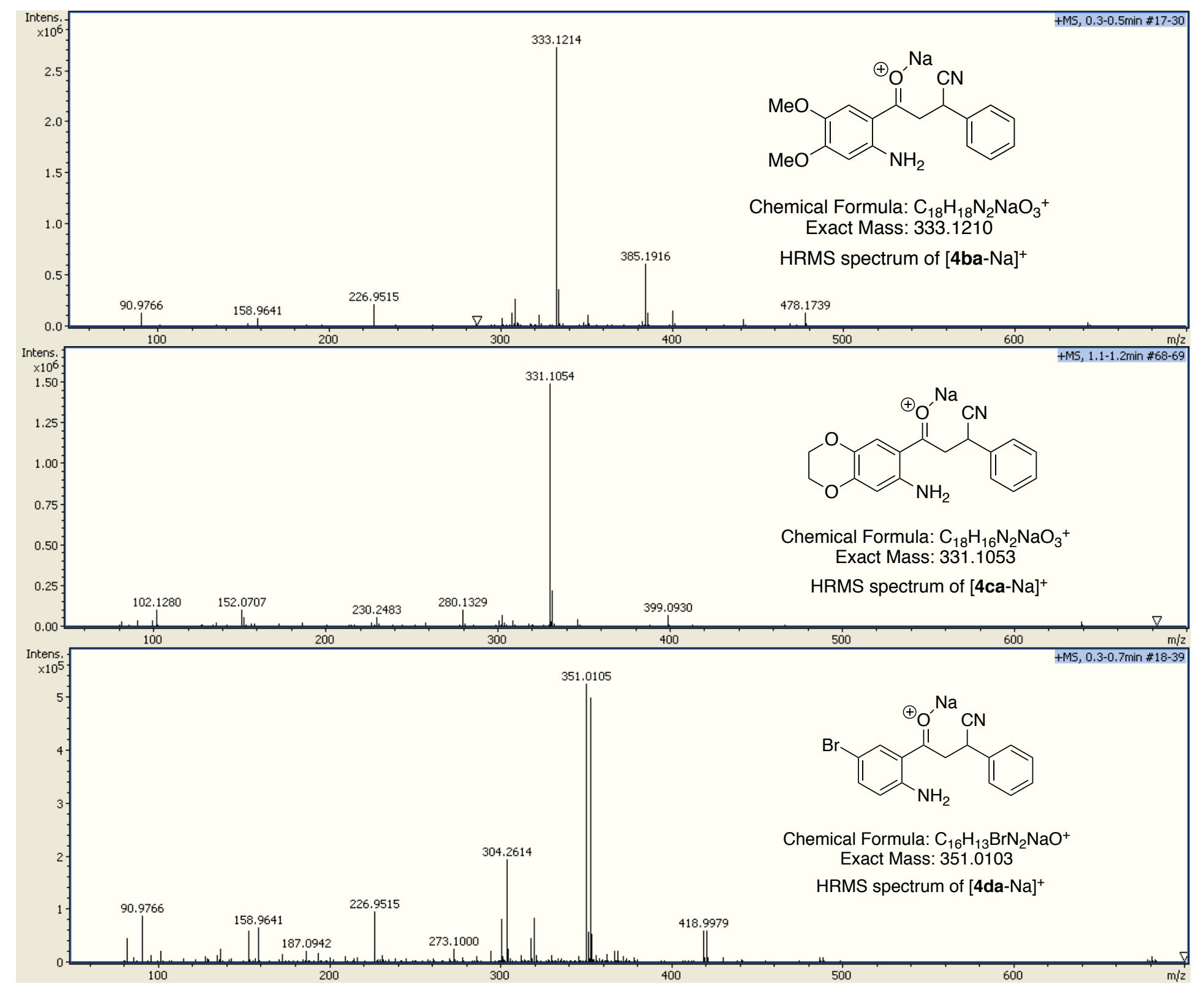




\section{HRMS Spectral Charts of 4-(2-nitrophenyl)-4-oxo-2-arylbutanenitriles 19}

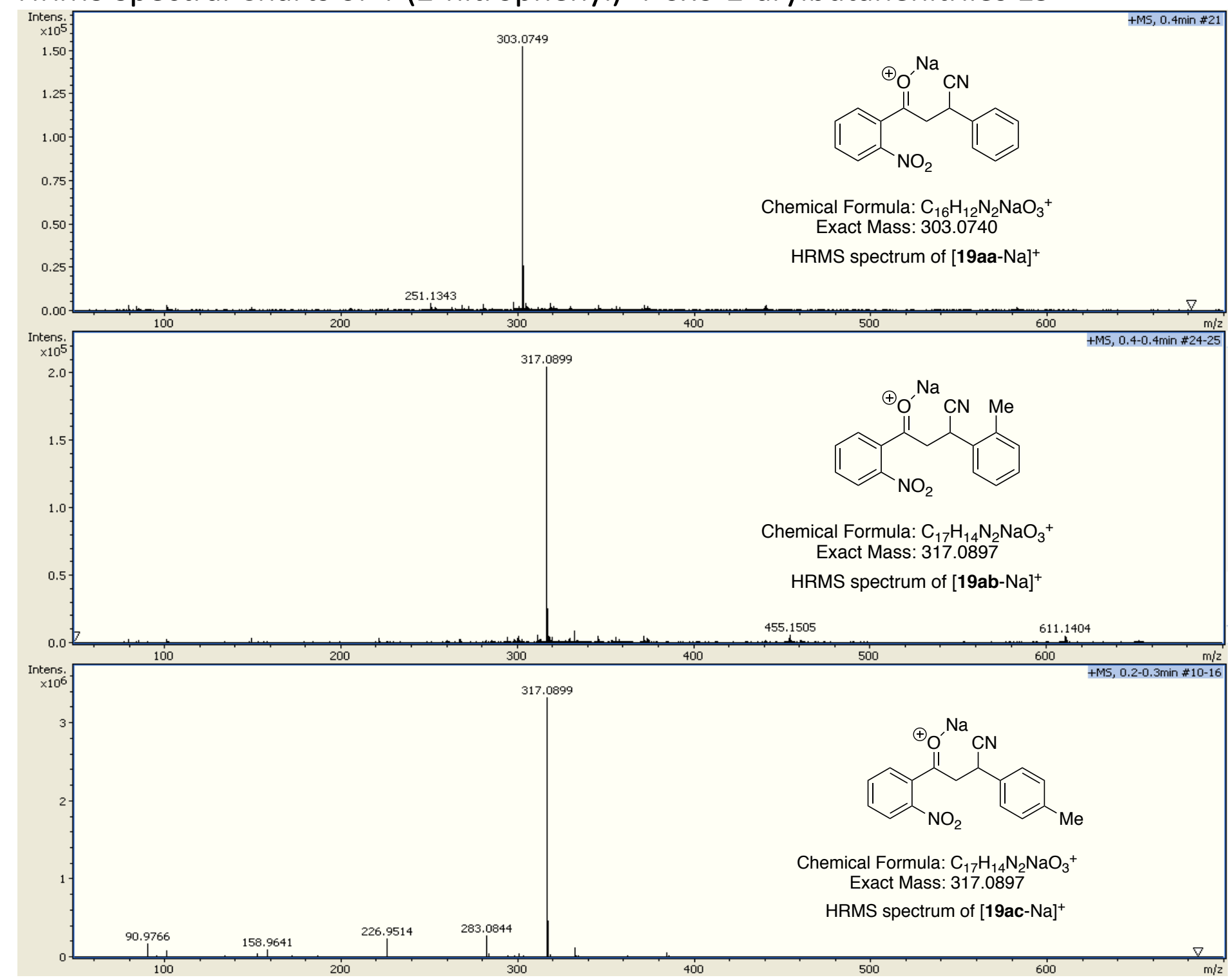




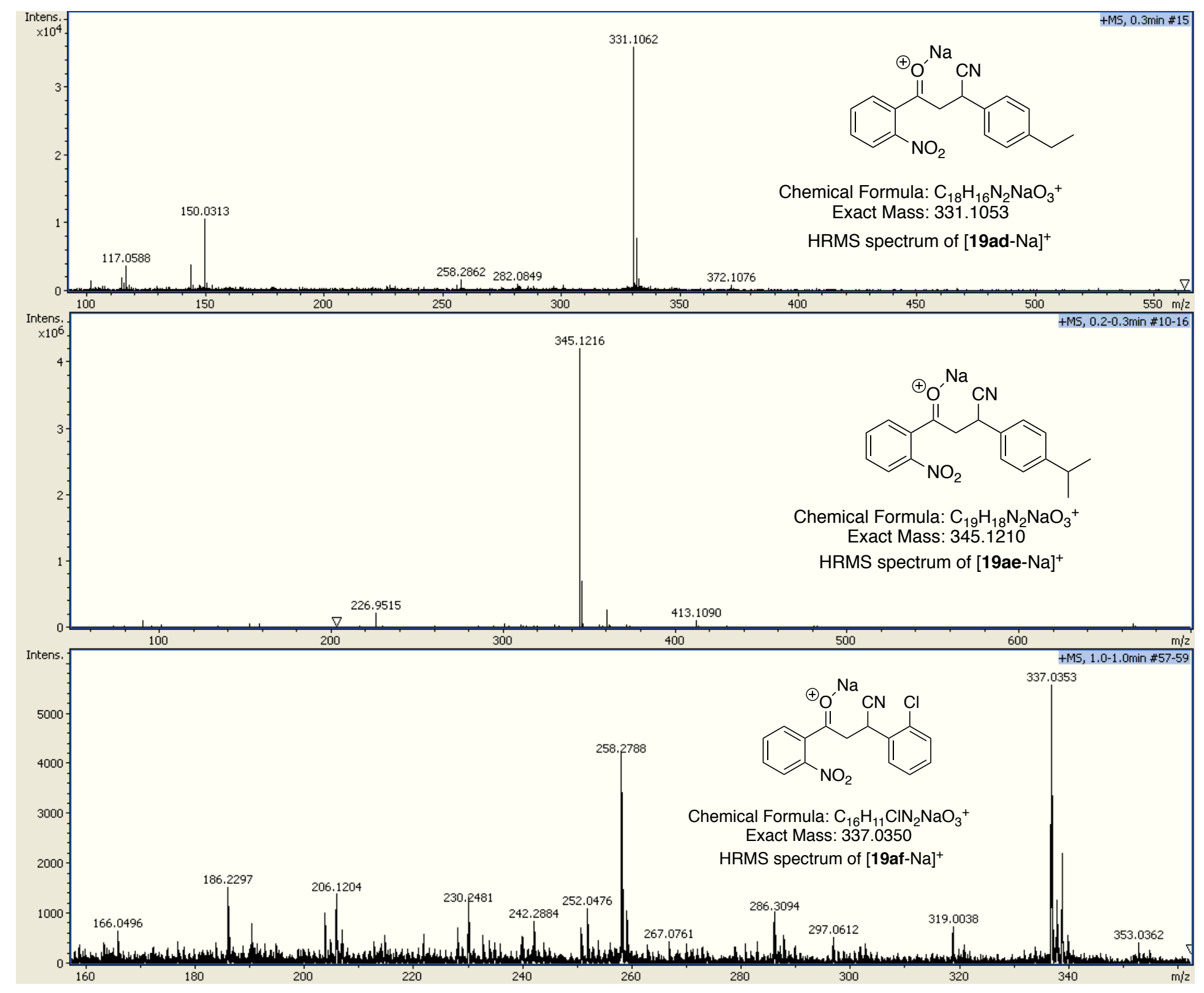




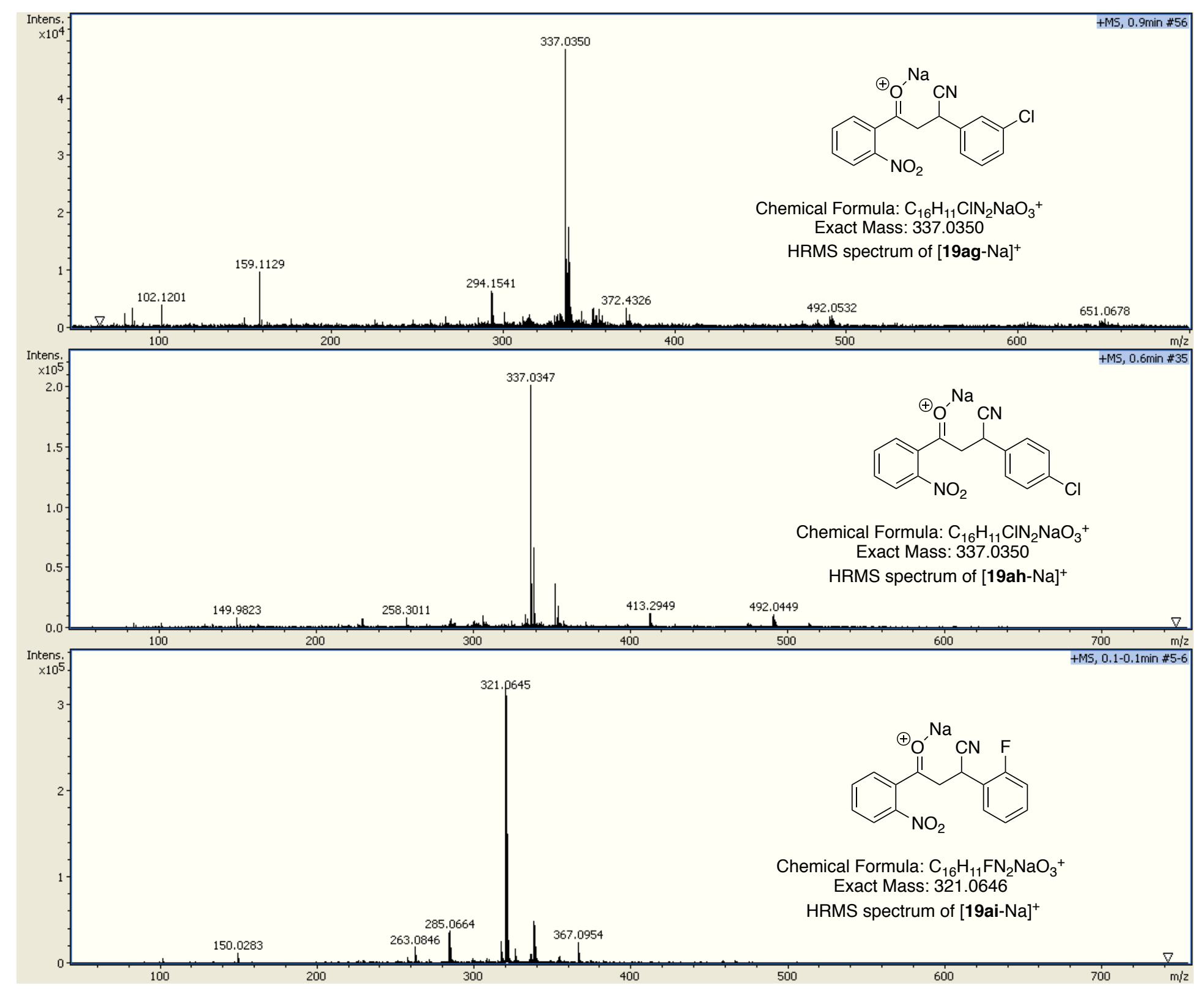




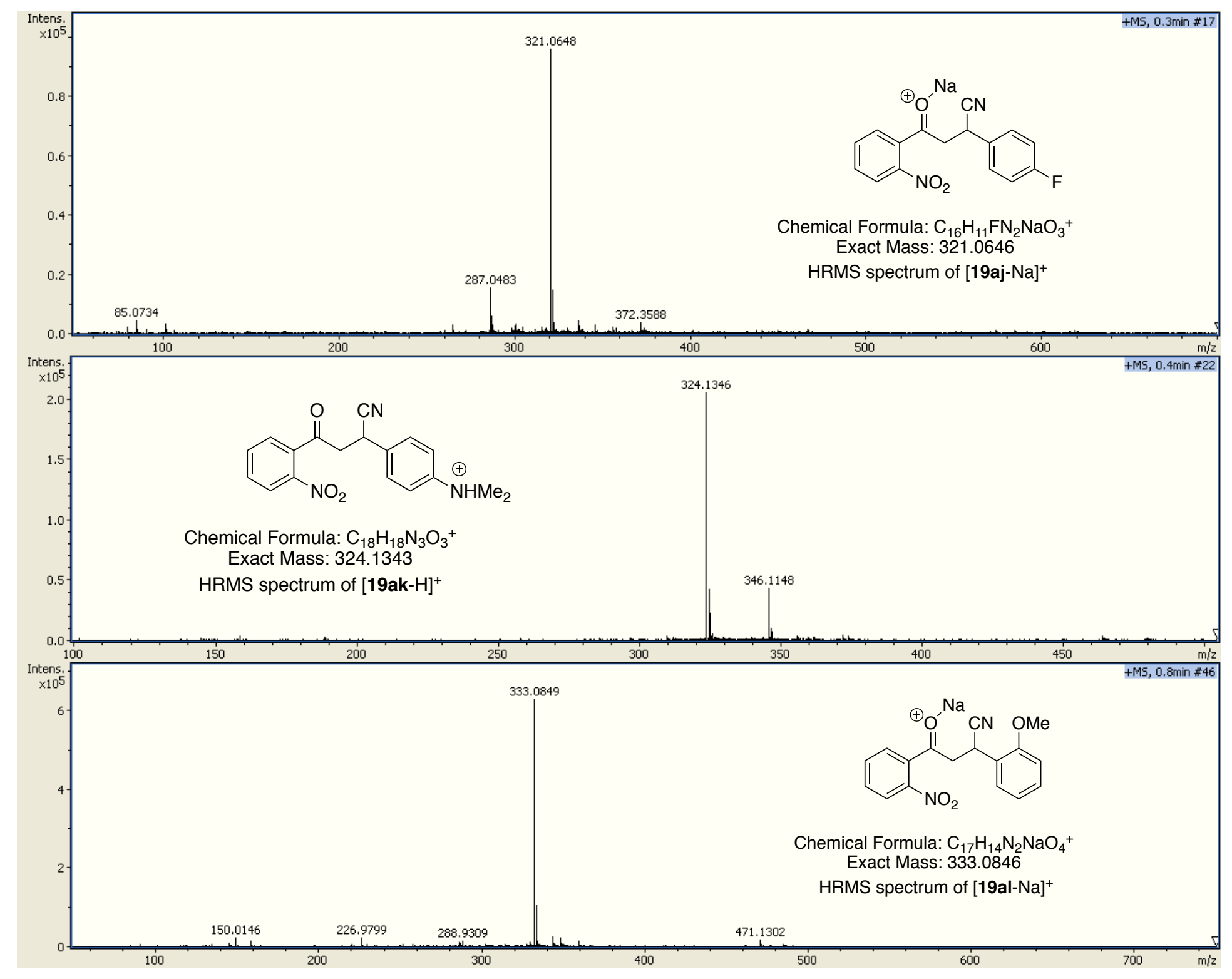




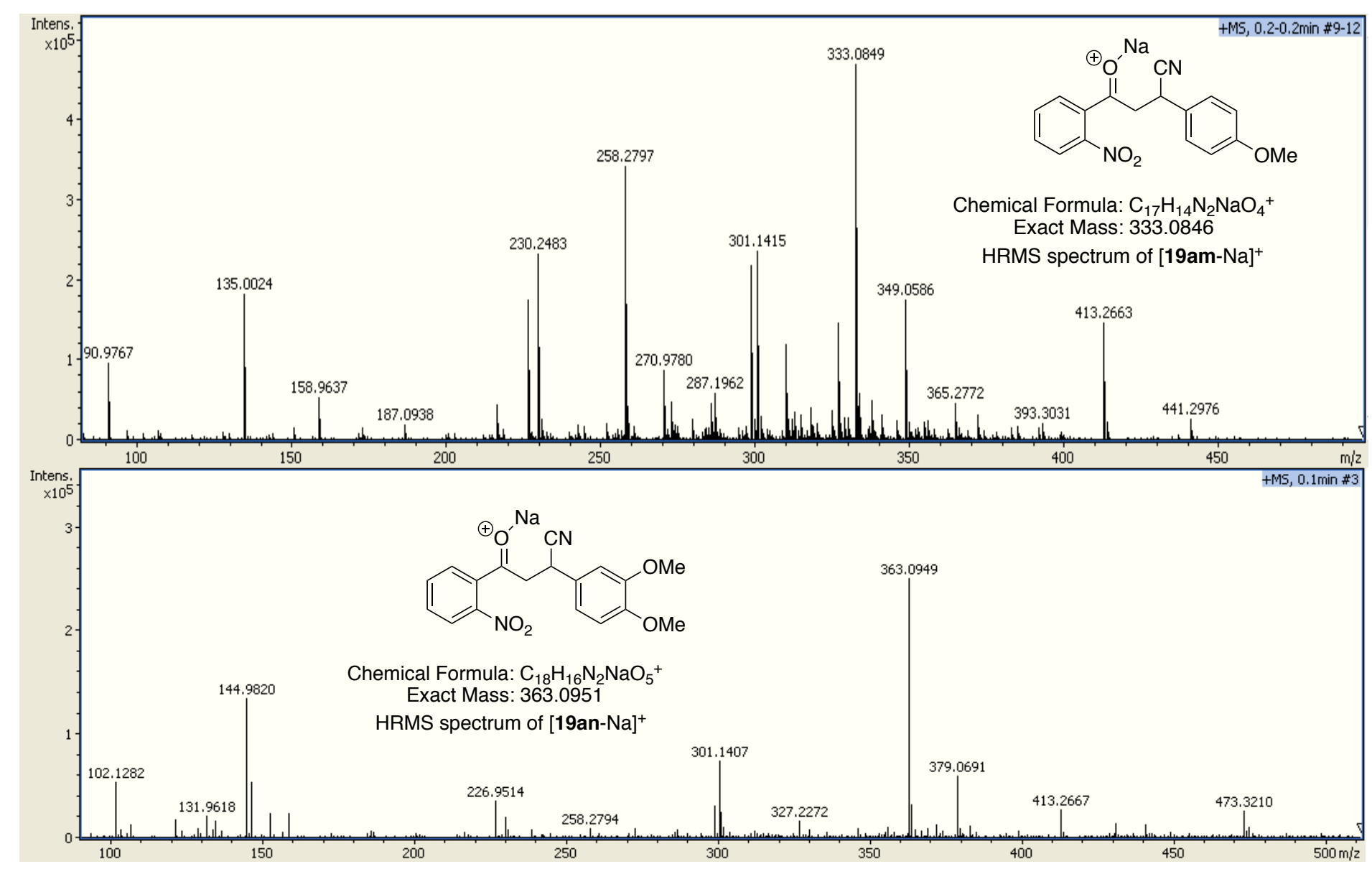




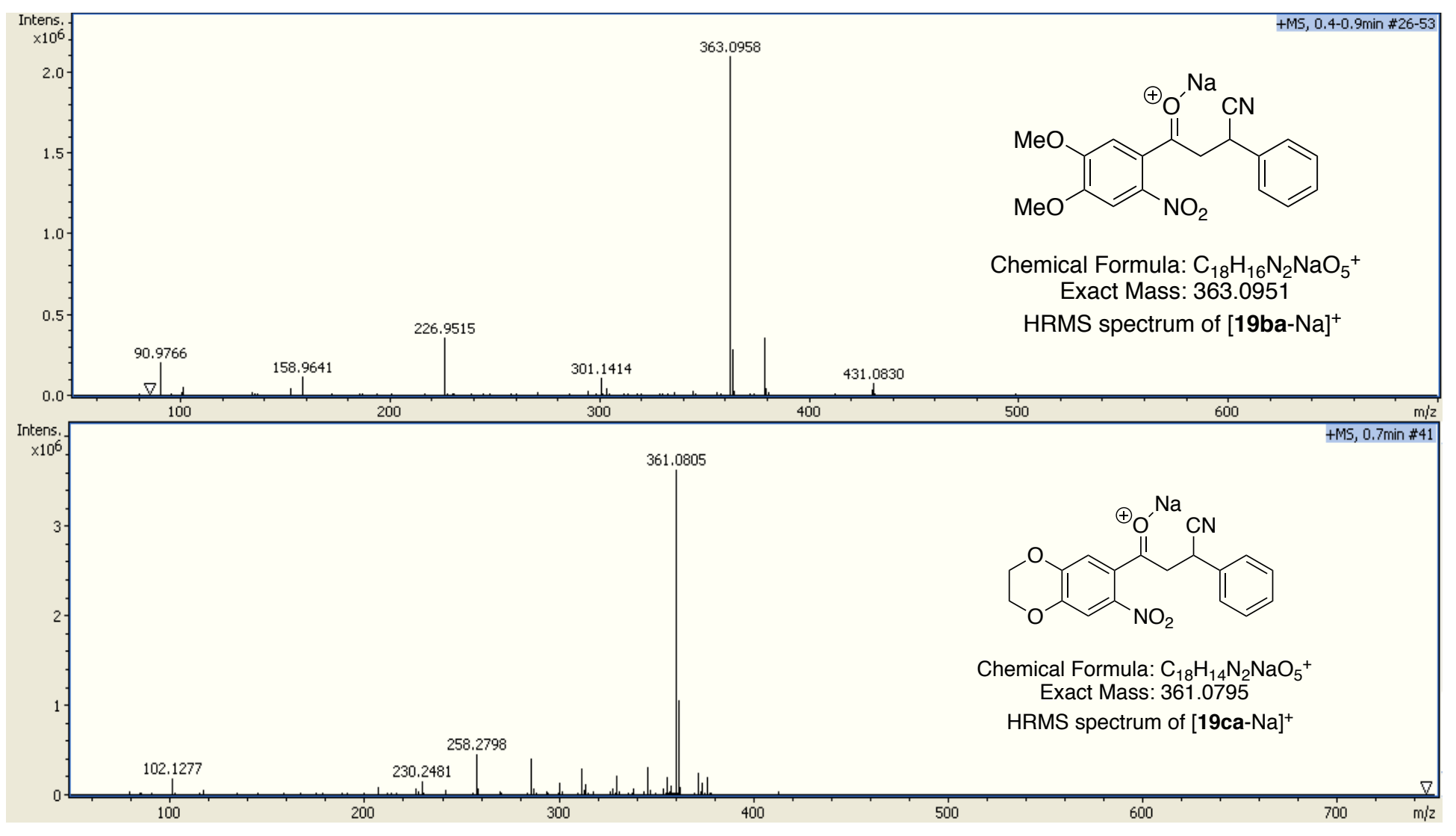




\section{HRMS Spectral Charts of (E)-1-(2-aminophenyl)-3-arylprop-2-en-1-ones 16}

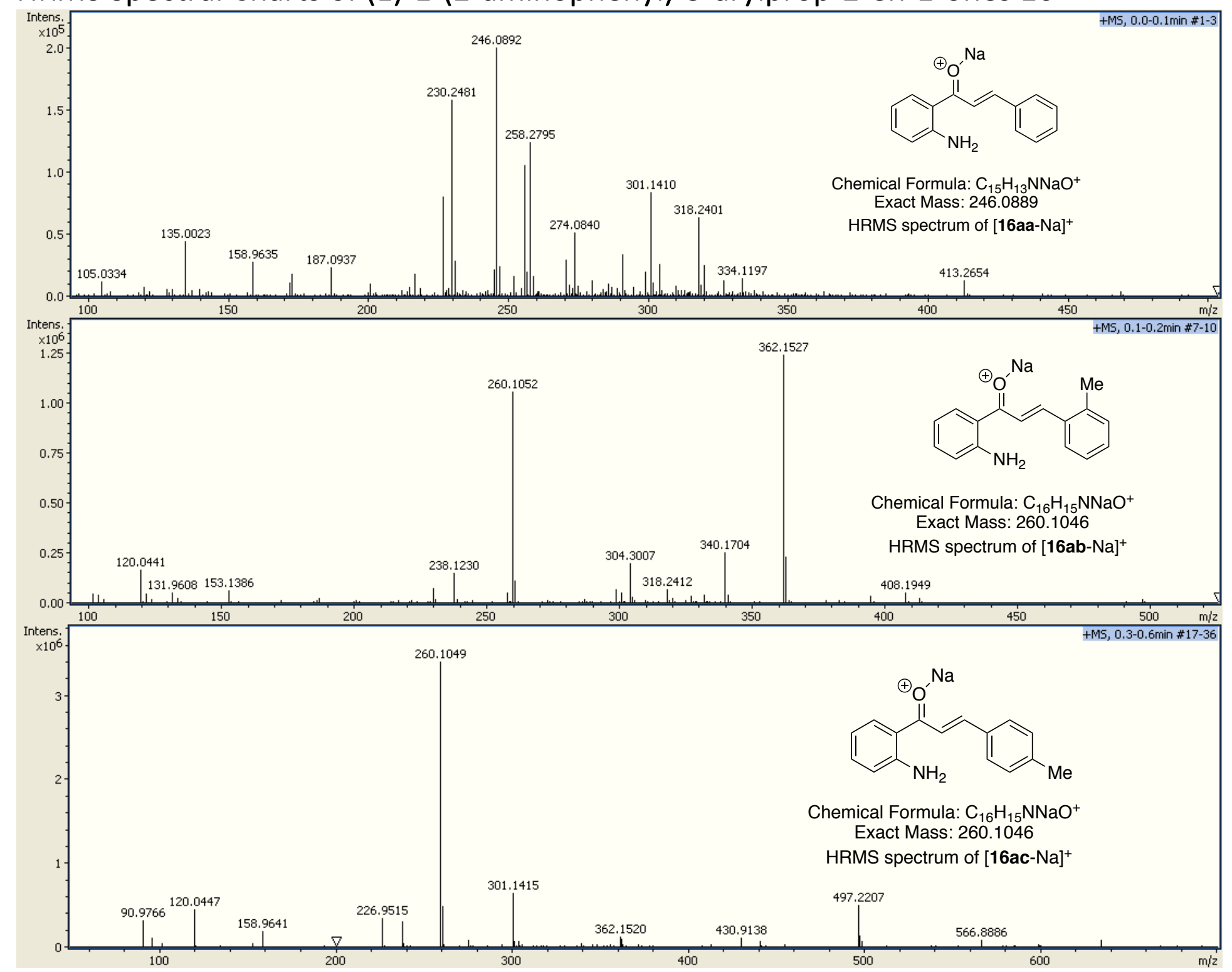




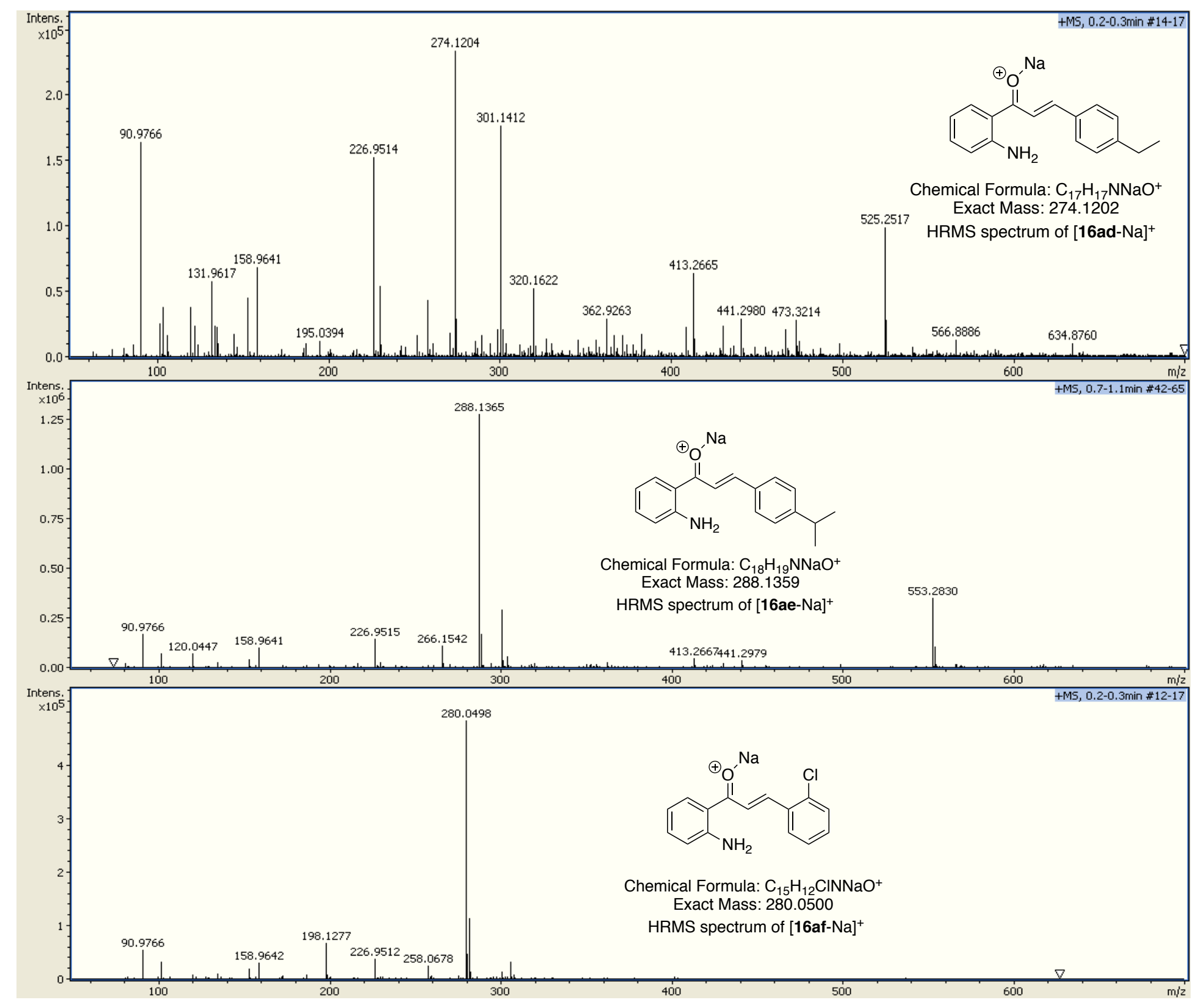




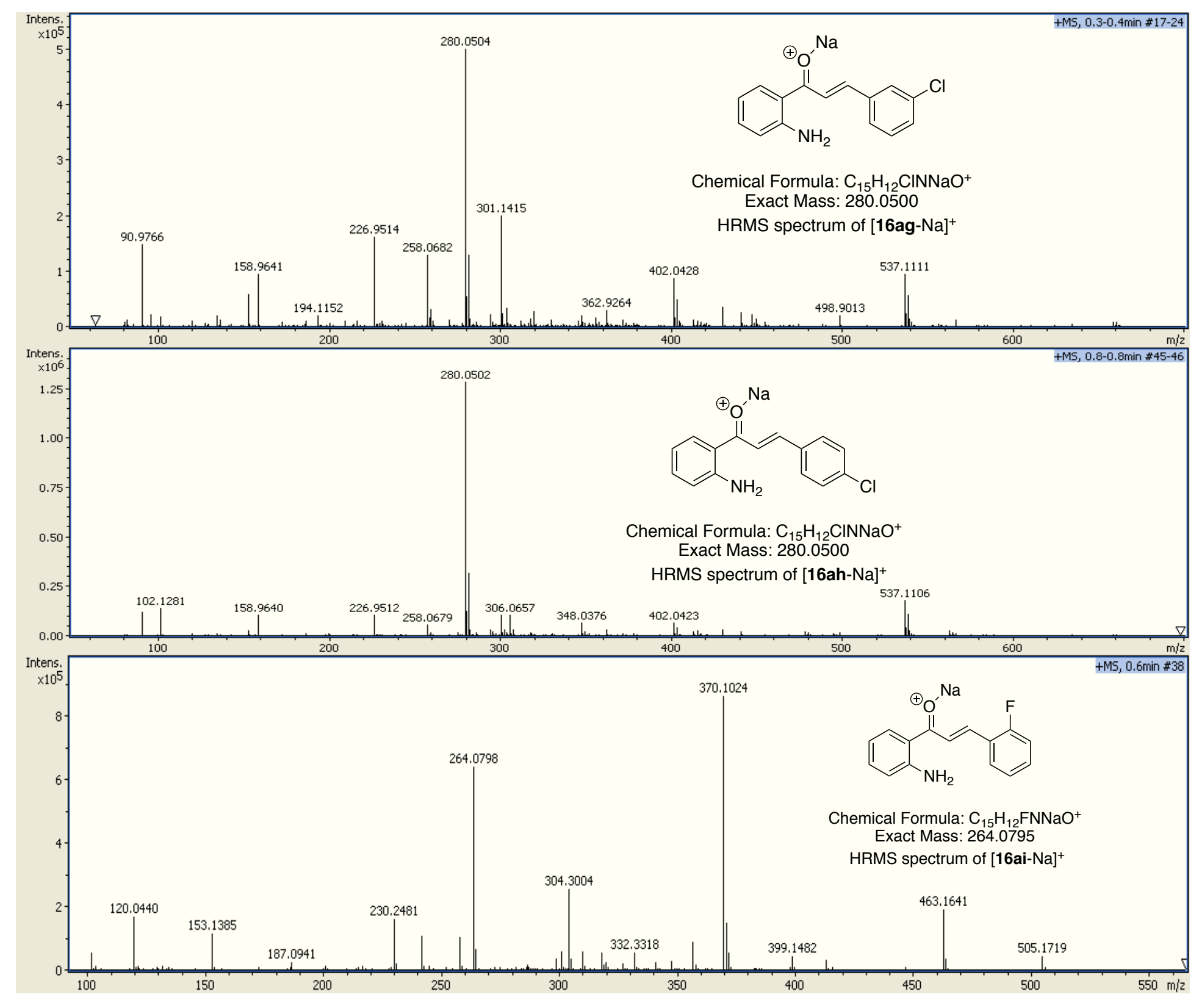




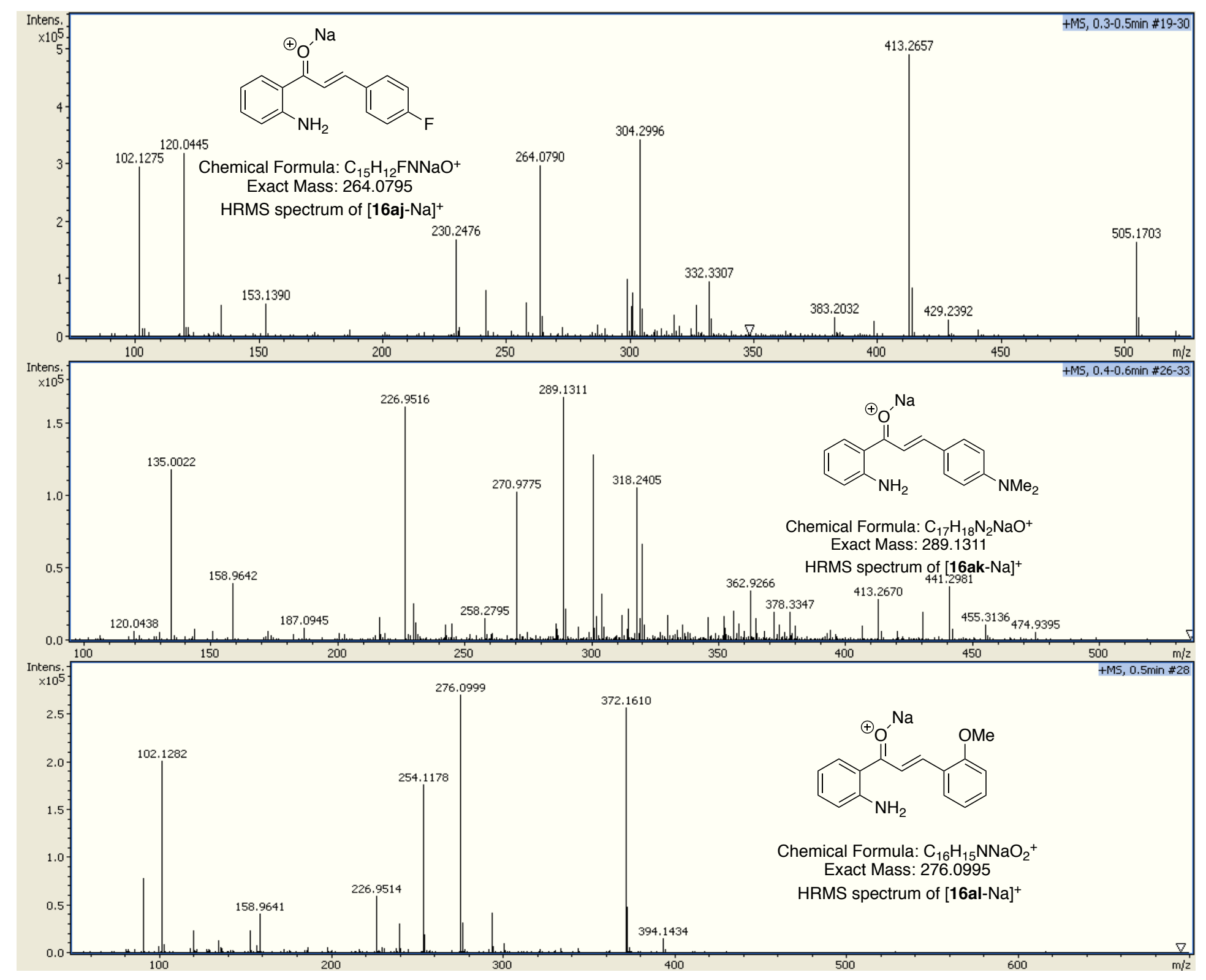




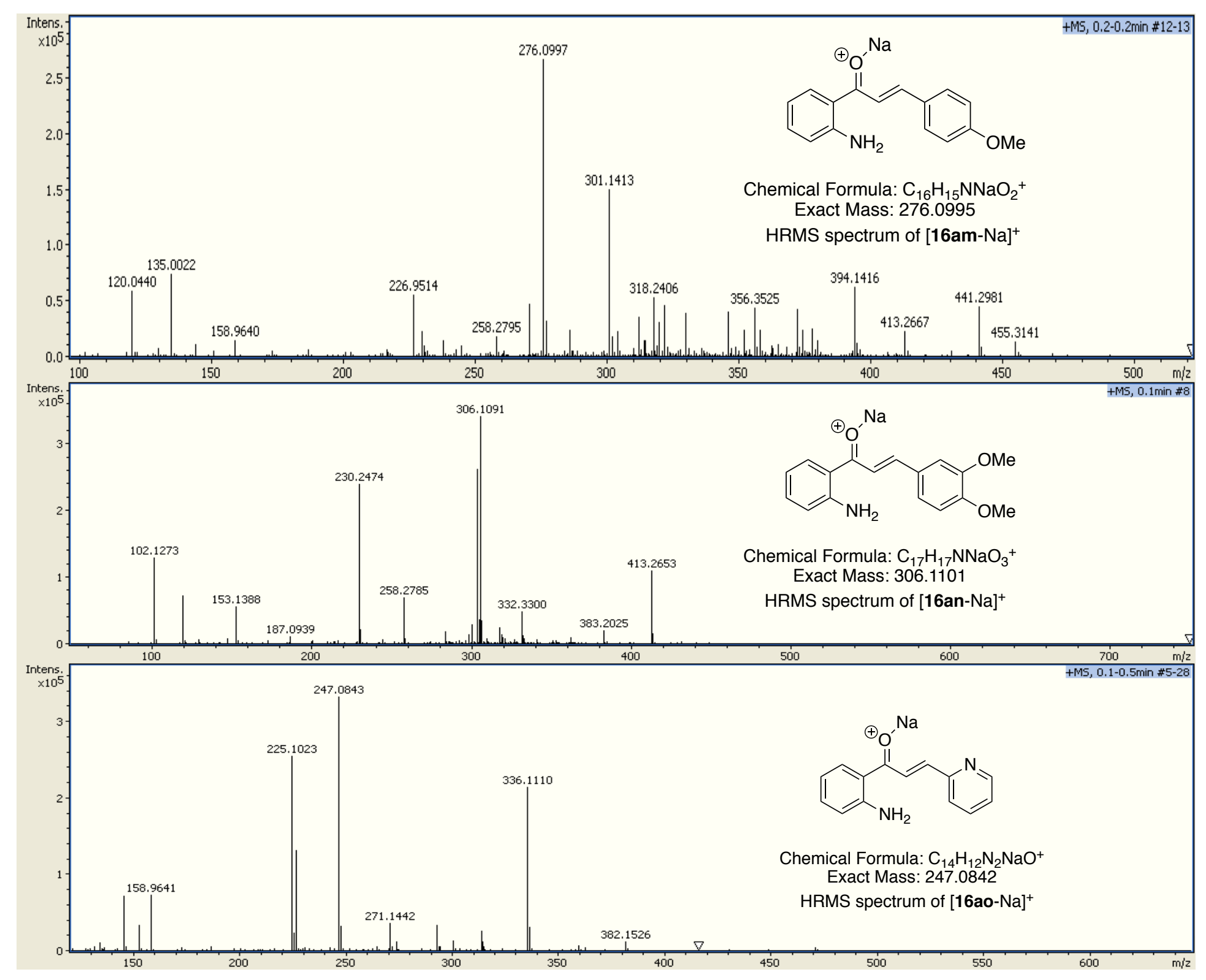




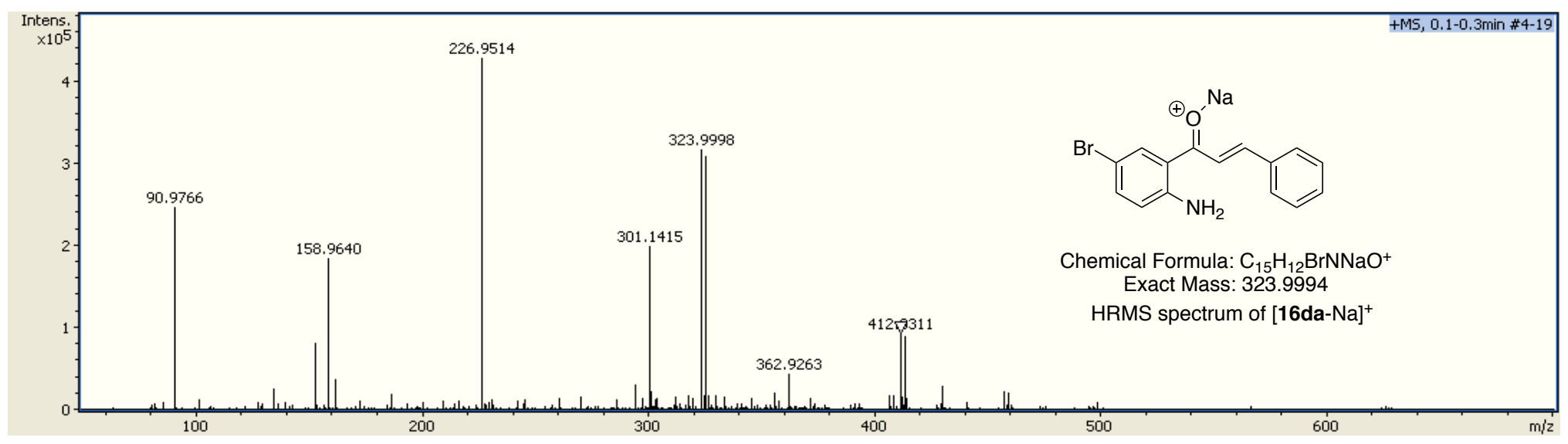

HRMS Spectral Charts of (E)-1-(2-nitrophenyl)-3-arylprop-2-en-1-ones 20

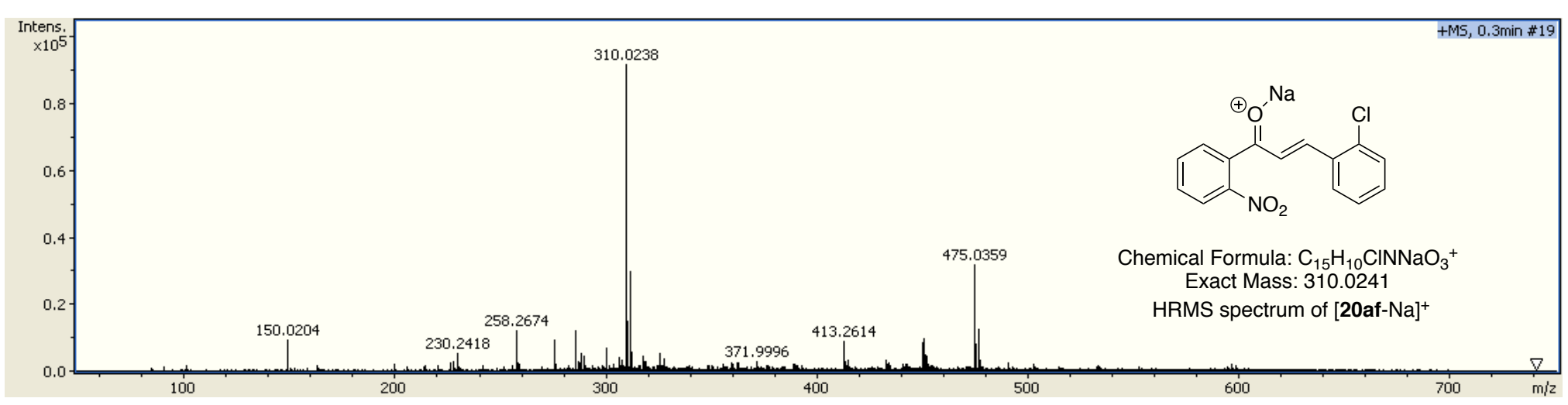




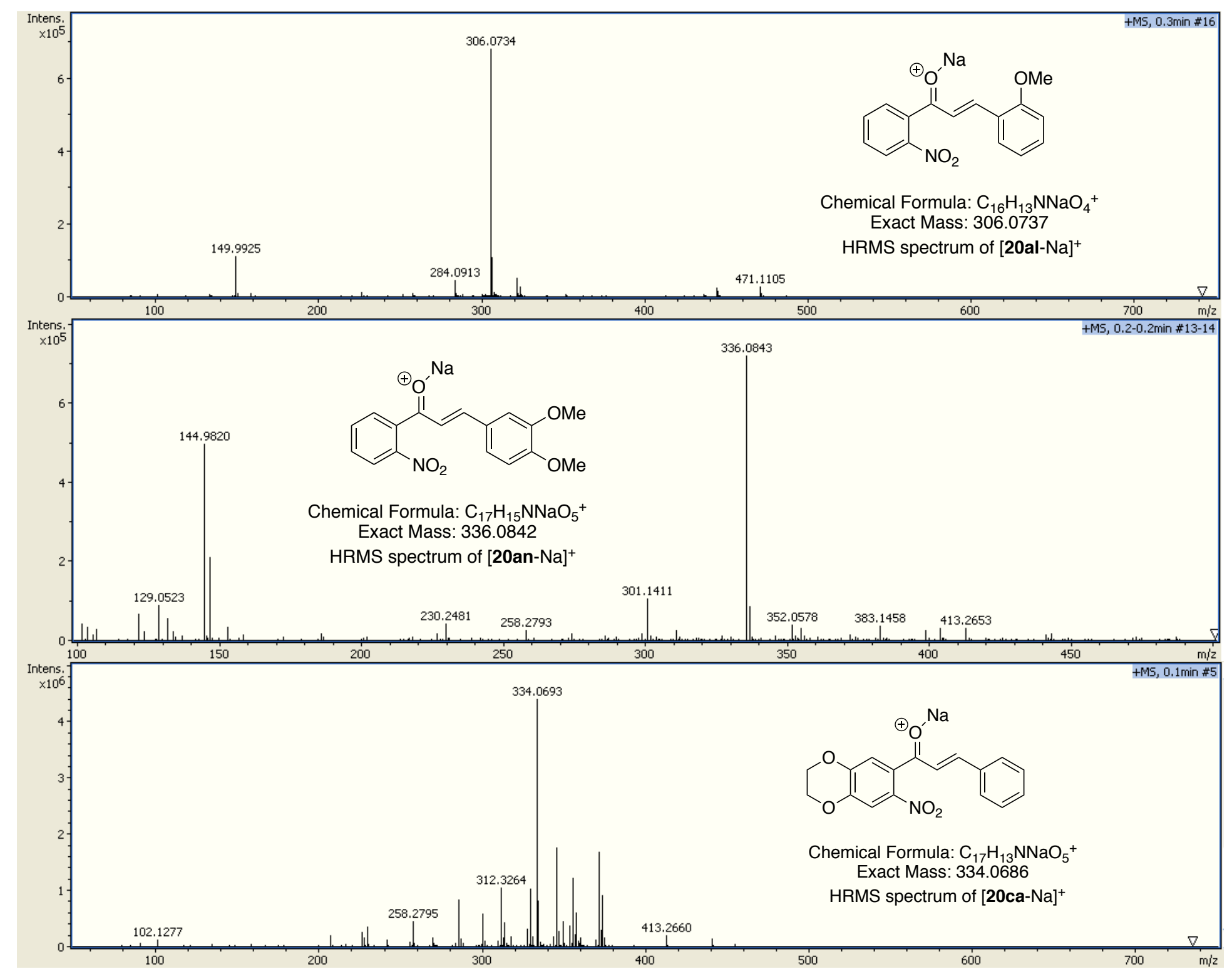



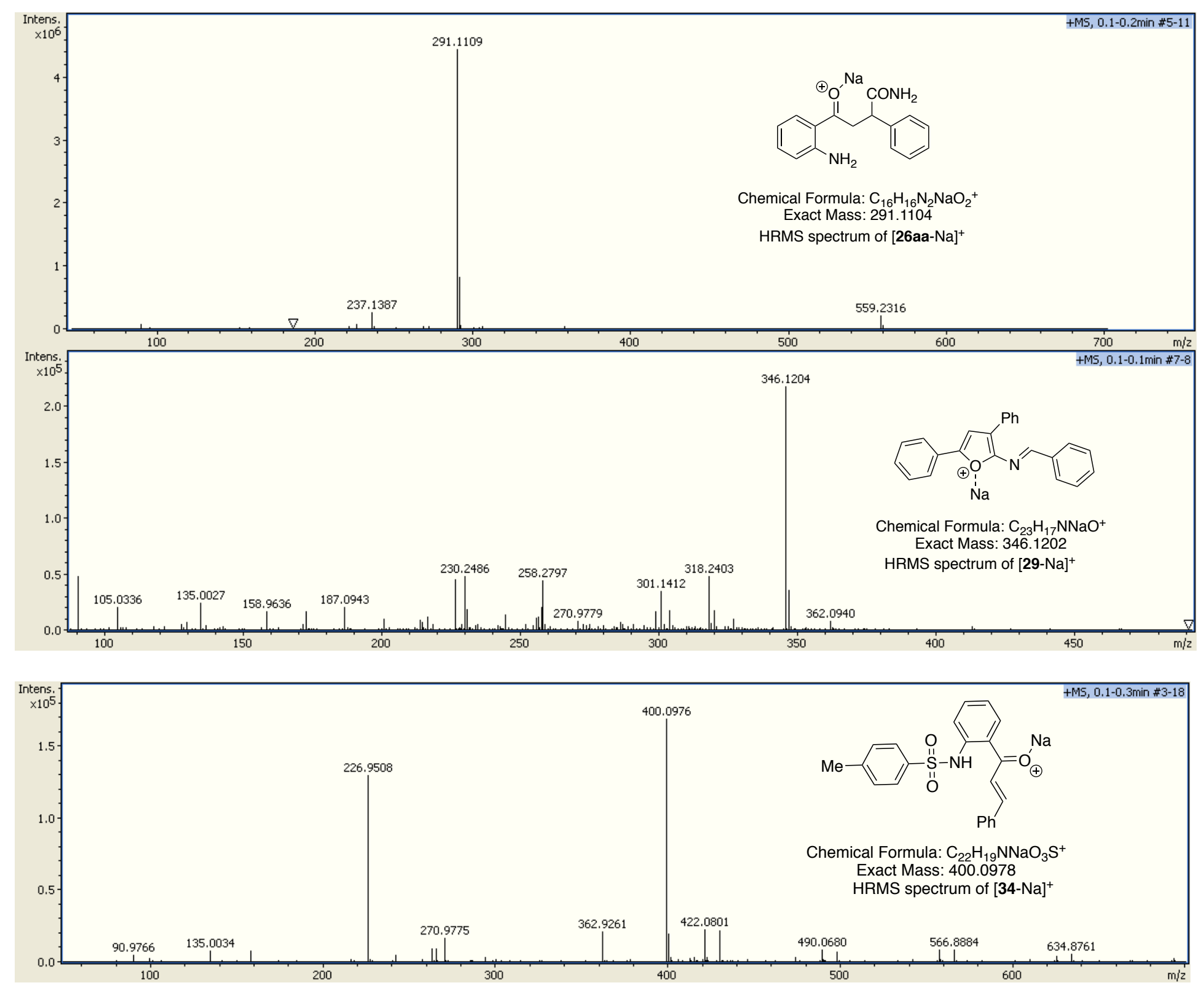

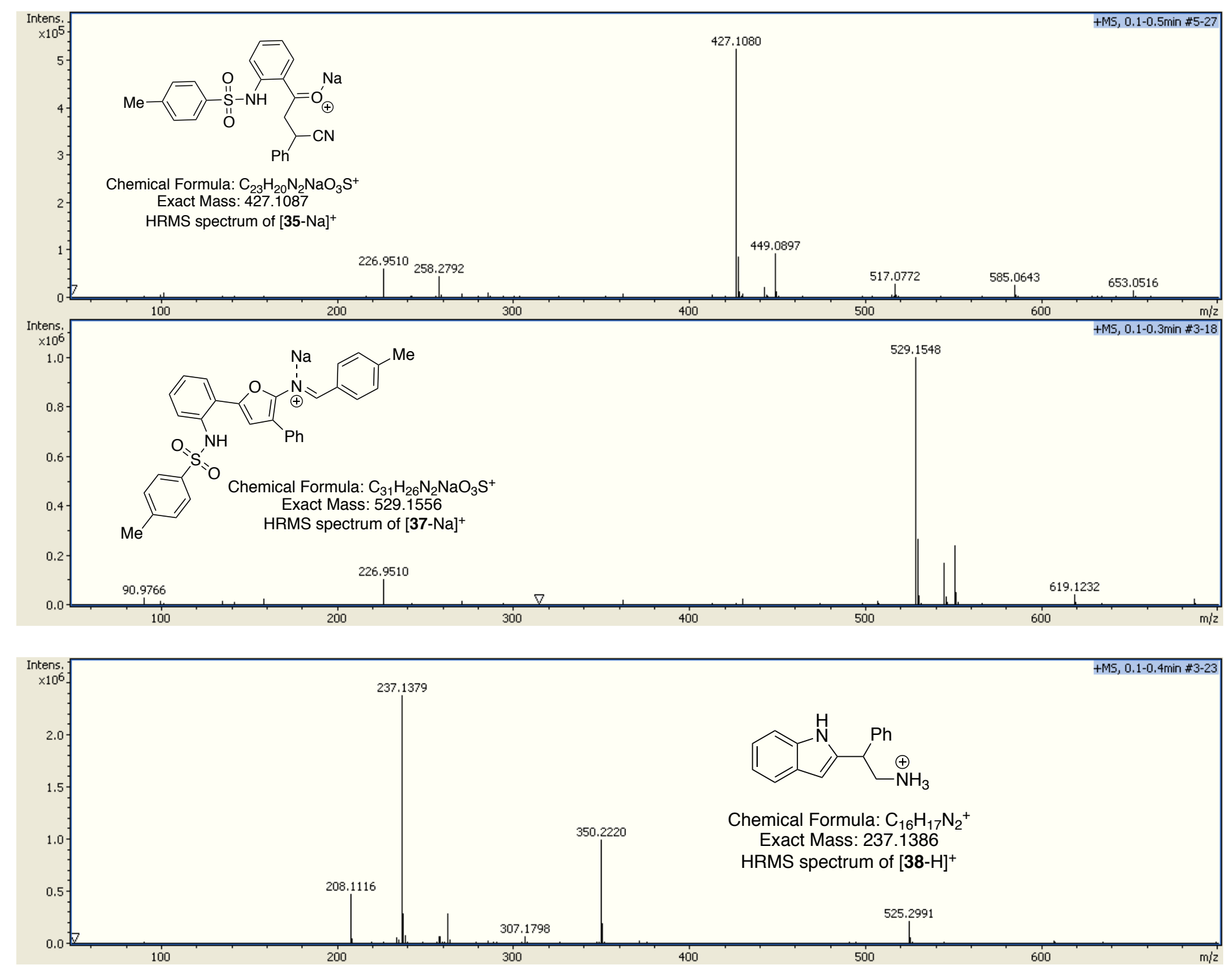


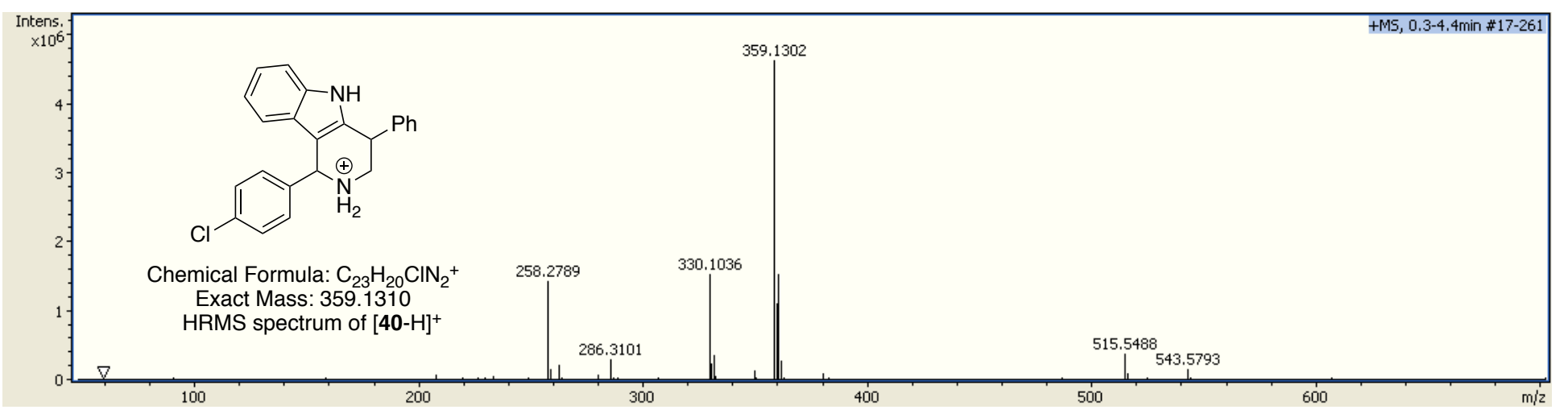


X-Ray Crystallography data for amine 38

\section{ORTEP Drawing}

Residue 1

Residue 2
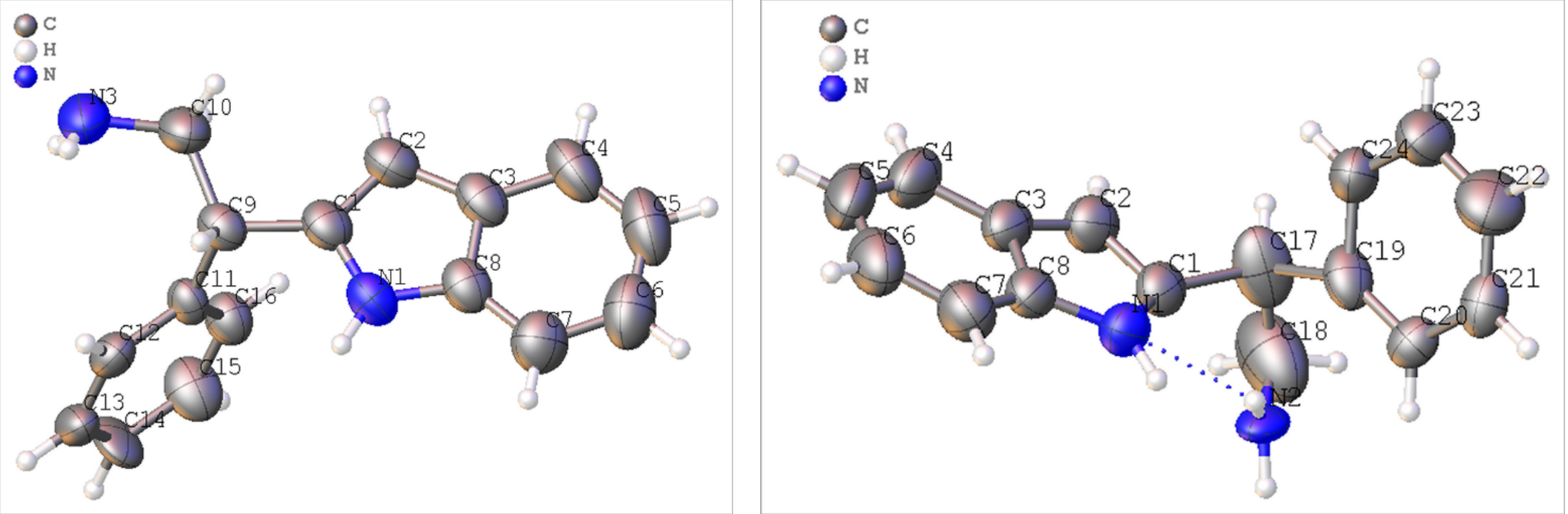

Sample Pictures of crystal

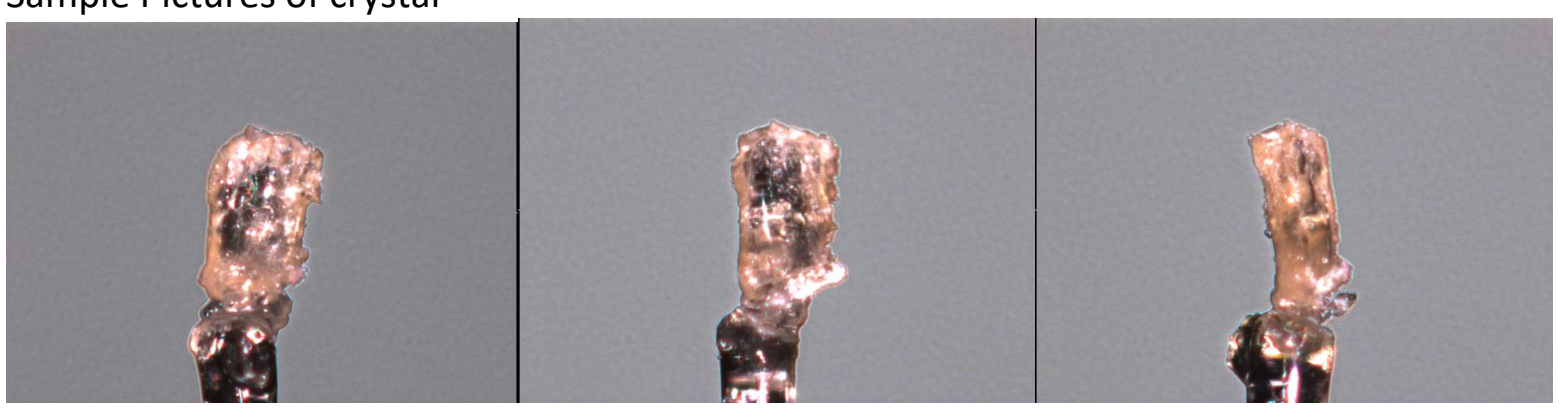


Table S1. Crystal data and structure refinement for 38.

\section{Identification code}

Empirical formula

Formula weight

Temperature/K

Crystal system

Space group

$\mathrm{a} / \AA$

$\mathrm{b} / \AA$

$\mathrm{c} / \AA$

$\alpha /{ }^{\circ}$

$\beta /{ }^{\circ}$

$\gamma /{ }^{\circ}$

Volume $/ \AA^{3}$

Z

$\rho_{\text {calc }} / \mathrm{cm}^{3}$

$\mu / \mathrm{mm}^{-1}$

$\mathrm{F}(000)$

Crystal size $/ \mathrm{mm}^{3}$

Radiation

$2 \Theta$ range for data collection $/{ }^{\circ}$

Index ranges

Reflections collected

Independent reflections

Data/restraints/parameters

Goodness-of-fit on $\mathrm{F}^{2}$

Final $\mathrm{R}$ indexes $[\mathrm{I}>=2 \sigma(\mathrm{I})]$

Final $\mathrm{R}$ indexes [all data]

Largest diff. peak/hole / e $\AA^{-3}$
ANNA1229 tryptamine 2

$\mathrm{C}_{16} \mathrm{H}_{16} \mathrm{~N}_{2}$

236.31

293(2)

orthorhombic

Pbca

$11.62377(13)$

$11.62379(12)$

$19.3359(2)$

90

90

90

2612.52(5)

8

1.202

0.550

1008.0

$0.421 \times 0.232 \times 0.121$

$\mathrm{CuK} \alpha(\lambda=1.54184)$

9.148 to 152.952

$-14 \leq \mathrm{h} \leq 14,-14 \leq \mathrm{k} \leq 12,-24 \leq 1 \leq 24$

31528

$2722\left[\mathrm{R}_{\text {int }}=0.0157, \mathrm{R}_{\text {sigma }}=0.0057\right]$

$2722 / 0 / 235$

1.045

$\mathrm{R}_{1}=0.0430, \mathrm{wR}_{2}=0.1305$

$\mathrm{R}_{1}=0.0466, \mathrm{wR}_{2}=0.1348$

$0.23 /-0.16$ 
Table S2. Fractional Atomic Coordinates $\left(\times 10^{4}\right)$ and Equivalent Isotropic Displacement Parameters $\left(\AA^{2} \times 10^{3}\right)$ for ANNA1229_tryptamine_2. U U is defined as 1/3 of of the trace of the orthogonalised UIJ tensor.

$$
\begin{aligned}
& \text {Attom }_{(1)} \\
& \mathrm{C}_{(11)} \\
& \mathrm{C}_{(12)} \\
& \mathrm{C}_{(13)} \\
& \mathrm{C}_{(14)} \\
& \mathrm{C}_{(15)} \\
& \mathrm{C}_{(16)} \\
& \mathrm{N}_{(3)} \\
& \mathrm{C}_{(8)} \\
& \mathrm{C}_{(3)} \\
& \mathrm{C}_{(1)} \\
& \mathrm{C}_{(2)} \\
& \mathrm{C}_{(9)} \\
& \mathrm{C}_{(7)} \\
& \mathrm{C}_{(4)} \\
& \mathrm{C}_{(10)} \\
& \mathrm{C}_{(5)} \\
& \mathrm{C}_{(6)} \\
& \mathrm{C}_{(17)} \\
& \mathrm{C}_{(18)} \\
& \mathrm{N}_{(2)} \\
& \mathrm{C}_{(19)} \\
& \mathrm{C}_{(24)} \\
& \mathrm{C}_{(23)} \\
& \mathrm{C}_{(22)} \\
& \mathrm{C}_{(21)} \\
& \mathrm{C}_{(20)}
\end{aligned}
$$

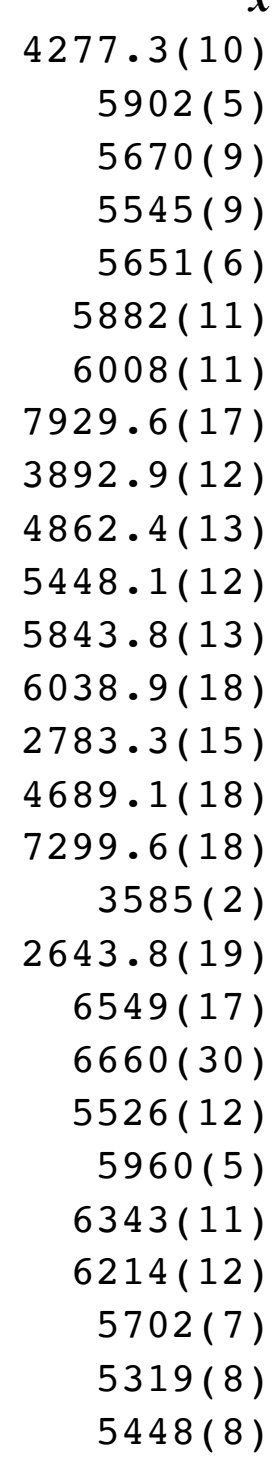

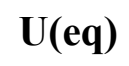


Table S3. Anisotropic Displacement Parameters $\left(\AA^{2} \times 10^{3}\right)$ for ANNA1229_tryptamine_2. The Anisotropic displacement factor exponent takes the form: $-2 \pi^{2}\left[h^{2} a * 2 U_{11}+2 h k a * b * U_{12}+\ldots\right]$.

\begin{tabular}{lr} 
Atom & \multicolumn{1}{c}{$\mathbf{U}_{11}$} \\
$\mathrm{~N}_{(1)}$ & $70.5(7)$ \\
$\mathrm{C}_{(11)}$ & $47(3)$ \\
$\mathrm{C}_{(12)}$ & $47(3)$ \\
$\mathrm{C}_{(13)}$ & $58(3)$ \\
$\mathrm{C}_{(14)}$ & $105(5)$ \\
$\mathrm{C}_{(15)}$ & $106(4)$ \\
$\mathrm{C}_{(16)}$ & $67(3)$ \\
$\mathrm{N}_{(3)}$ & $82.5(10)$ \\
$\mathrm{C}_{(8)}$ & $74.6(8)$ \\
$\mathrm{C}_{(3)}$ & $83.6(9)$ \\
$\mathrm{C}_{(1)}$ & $70.5(8)$ \\
$\mathrm{C}_{(2)}$ & $70.3(8)$ \\
$\mathrm{C}_{(9)}$ & $63.6(10)$ \\
$\mathrm{C}_{(7)}$ & $78.2(10)$ \\
$\mathrm{C}_{(4)}$ & $119.3(13)$ \\
$\mathrm{C}_{(10)}$ & $75.3(12)$ \\
$\mathrm{C}_{(5)}$ & $142.7(17)$ \\
$\mathrm{C}_{(6)}$ & $108.2(14)$ \\
$\mathrm{C}_{(17)}$ & $51(9)$ \\
$\mathrm{C}_{(18)}$ & $120(20)$ \\
$\mathrm{N}_{(2)}$ & $101(9)$ \\
$\mathrm{C}_{(19)}$ & $71(4)$ \\
$\mathrm{C}_{(24)}$ & $67(4)$ \\
$\mathrm{C}_{(23)}$ & $102(4)$ \\
$\mathrm{C}_{(22)}$ & $54(3)$ \\
$\mathrm{C}_{(21)}$ & \\
$\mathrm{C}_{(20)}$ & \\
& $120(7)$ \\
& \\
& $142)$ \\
&
\end{tabular}

$\mathbf{U}_{22}$

$46.4(6)$ $38(2)$

$58(2)$

$51(2)$

$27(3)$

$66(3)$

$49.8(19)$

$58.8(9)$

$49.6(7)$

$44.8(7)$

$44.6(6)$

$51.6(7)$

$44.9(7)$

75.3(10)

$47.9(7)$

$53.1(9)$

$60.2(9)$

$85.7(12)$

$67(10)$

$140(20)$

$50(6)$

$52(3)$

$47.6(18)$

$63(3)$

$94(7)$

44.7 ( 19)

$51(2)$
$\mathbf{U}_{33}$

$60.4(6)$

48 (3)

$56(2)$

77 (3)

$82(4)$

$50(2)$

$50.2(18)$

$86.8(11)$

$49.3(6)$

$51.7(6)$

$53.4(6)$

$66.3(8)$

$45.0(7)$

$75.1(9)$

$69.1(9)$

$75.4(11)$

$73.9(10)$

$77.2(10)$

$132(18)$

$120(20)$

$25(4)$

$59(4)$

$64(2)$

$63(3)$

$62(3)$

$72(3)$

$48.3(18)$
$\mathbf{U}_{23}$

$-1.0(5)$ $-8(2)$

$-5.1(16)$

$-13.7(17)$

$17(2)$

$10.0(18)$

$-2.4(13)$

$2.5(8)$

4.1 (5)

$4.2(5)$

$7.9(5)$

$6.1(6)$

$1.1(5)$

$4.7(8)$

$-3.8(7)$

$2.1(8)$

$-11.0(8)$

$-0.1(9)$

$23(11)$

31 (18)

0 (4)

$17(3)$

$-2.5(15)$

$8(2)$

15 ( 3$)$

-1.5 ( 15$)$

$-5.9(15)$
$\mathbf{U}_{13}$

$6.8(5)$

$5(2)$

$-12.6(17)$

$-27(2)$

$-17(3)$

9 (2)

$2.9(17)$

$-31.5(9)$

4.7 ( 5$)$

5.2 ( 6 )

$0.1(5)$

$2.1(6)$

$2.0(6)$

$5.2(7)$

$6.3(9)$

$-20.2(9)$

$1.3(11)$

$-3.9(10)$

$-12(10)$

$-31(18)$

14 ( 4 )

$-20(3)$

$5(2)$

$10(2)$

$-9(3)$

$-22(2)$

$-9.8(15)$
$\mathrm{U}_{12}$

$2.4(5)$

$0.6(18)$

$0.1(16)$

$11.1(17)$

$3(2)$

$-7(2)$

$-1.4(17)$

$2.5(7)$

$-3.7(6)$

$2.4(6)$

$1.3(5)$

$7.4(6)$

$5.1(7)$

$-11.4(8)$

$3.7(8)$

$10.4(8)$

$-25.8(10)$

$-35.4(11)$

$11(7)$

$-51(19)$

$1(5)$

$-10(3)$

$-4.1(18)$

$-1(2)$

$-2(5)$

$1.2(14)$

3. 7 (14) 
Table S4. Bond Lengths for ANNA1229_tryptamine_2.

\begin{tabular}{|c|c|c|c|c|c|}
\hline \multicolumn{2}{|c|}{ Atom Atom } & \multirow{2}{*}{$\begin{array}{c}\text { Length/Å } \\
1.3705(17)\end{array}$} & \multicolumn{2}{|c|}{ Atom Atom } & \multirow{2}{*}{$\begin{array}{c}\text { Length/Å } \\
1.5044(19)\end{array}$} \\
\hline $\mathrm{N}_{(1)}$ & $\mathrm{C}_{(8)}$ & & $\mathrm{C}_{(1)}$ & $\mathrm{C}_{(9)}$ & \\
\hline $\mathrm{N}_{(1)}$ & $\mathrm{C}_{(1)}$ & $1.3628(18)$ & $\mathrm{C}_{(1)}$ & $\mathrm{C}_{(17)}$ & $1.604(18)$ \\
\hline $\mathrm{C}_{(11)}$ & $\mathrm{C}_{(12)}$ & 1.3900 & $\mathrm{C}_{(9)}$ & $\mathrm{C}_{(10)}$ & $1.530(3)$ \\
\hline $\mathrm{C}_{(11)}$ & $\mathrm{C}_{(16)}$ & 1.3900 & $\mathrm{C}_{(7)}$ & $\mathrm{C}_{(6)}$ & $1.379(2)$ \\
\hline $\mathrm{C}_{(11)}$ & $\mathrm{C}_{(9)}$ & $1.485(5)$ & $\mathrm{C}_{(4)}$ & $\mathrm{C}_{(5)}$ & $1.377(3)$ \\
\hline $\mathrm{C}_{(12)}$ & $\mathrm{C}_{(13)}$ & 1.3900 & $\mathrm{C}_{(5)}$ & $\mathrm{C}_{(6)}$ & $1.388(3)$ \\
\hline $\mathrm{C}_{(13)}$ & $\mathrm{C}_{(14)}$ & 1.3900 & $\mathrm{C}_{(17)}$ & $\mathrm{C}_{(18)}$ & $1.43(5)$ \\
\hline $\mathrm{C}_{(14)}$ & $\mathrm{C}_{(15)}$ & 1.3900 & $\mathrm{C}_{(17)}$ & $\mathrm{C}_{(19)}$ & $1.715(17)$ \\
\hline $\mathrm{C}_{(15)}$ & $\mathrm{C}_{(16)}$ & 1.3900 & $\mathrm{C}_{(18)}$ & $\mathrm{N}_{(2)}$ & $1.40(4)$ \\
\hline $\mathrm{N}_{(3)}$ & $\mathrm{C}_{(10)}$ & $1.452(2)$ & $\mathrm{C}_{(19)}$ & $\mathrm{C}_{(24)}$ & 1.3900 \\
\hline $\mathrm{C}_{(8)}$ & $\mathrm{C}_{(3)}$ & $1.407(2)$ & $\mathrm{C}_{(19)}$ & $\mathrm{C}_{(20)}$ & 1.3900 \\
\hline $\mathrm{C}_{(8)}$ & $\mathrm{C}_{(7)}$ & $1.384(2)$ & $\mathrm{C}_{(24)}$ & $\mathrm{C}_{(23)}$ & 1.3900 \\
\hline $\mathrm{C}_{(3)}$ & $\mathrm{C}_{(2)}$ & $1.429(2)$ & $\mathrm{C}_{(23)}$ & $\mathrm{C}_{(22)}$ & 1.3900 \\
\hline $\mathrm{C}_{(3)}$ & $\mathrm{C}_{(4)}$ & $1.3981(19)$ & $\mathrm{C}_{(22)}$ & $\mathrm{C}_{(21)}$ & 1.3900 \\
\hline $\mathrm{C}_{(1)}$ & $\mathrm{C}_{(2)}$ & $1.3678(18)$ & $\mathrm{C}_{(21)}$ & $\mathrm{C}_{(20)}$ & 1.3900 \\
\hline
\end{tabular}


Table S5. Bond Angles for ANNA1229_tryptamine_2.

\begin{tabular}{|c|c|c|c|c|c|c|c|}
\hline \multicolumn{3}{|c|}{ Atom Atom Atom } & Angle ${ }^{\circ}$ & \multicolumn{3}{|c|}{ Atom Atom Atom } & \multirow{2}{*}{$\begin{array}{r}\text { Angle } /^{\circ} \\
110.4(3)\end{array}$} \\
\hline $\mathrm{C}_{(1)}$ & $\mathrm{N}_{(1)}$ & $\mathrm{C}_{(8)}$ & $109.53(11)$ & $\mathrm{C}_{(11)}$ & $\mathrm{C}_{(9)}$ & $\mathrm{C}_{(1)}$ & \\
\hline $\mathrm{C}_{(12)}$ & $\mathrm{C}_{(11)}$ & $\mathrm{C}_{(16)}$ & 120.0 & $\mathrm{C}_{(11)}$ & $\mathrm{C}_{(9)}$ & $\mathrm{C}_{(10)}$ & $112.8(3)$ \\
\hline $\mathrm{C}_{(12)}$ & $\mathrm{C}_{(11)}$ & $\mathrm{C}_{(9)}$ & $117.6(3)$ & $\mathrm{C}_{(1)}$ & $\mathrm{C}_{(9)}$ & $\mathrm{C}_{(10)}$ & $109.95(14)$ \\
\hline $\mathrm{C}_{(16)}$ & $\mathrm{C}_{(11)}$ & $\mathrm{C}_{(9)}$ & $122.4(3)$ & $\mathrm{C}_{(6)}$ & $\mathrm{C}_{(7)}$ & $\mathrm{C}_{(8)}$ & $117.80(17)$ \\
\hline $\mathrm{C}_{(13)}$ & $\mathrm{C}_{(12)}$ & $\mathrm{C}_{(11)}$ & 120.0 & $\mathrm{C}_{(5)}$ & $\mathrm{C}_{(4)}$ & $\mathrm{C}_{(3)}$ & $119.18(17)$ \\
\hline $\mathrm{C}_{(12)}$ & $\mathrm{C}_{(13)}$ & $\mathrm{C}_{(14)}$ & 120.0 & $\mathrm{~N}_{(3)}$ & $\mathrm{C}_{(10)}$ & $\mathrm{C}_{(9)}$ & $115.64(15)$ \\
\hline $\mathrm{C}_{(15)}$ & $\mathrm{C}_{(14)}$ & $\mathrm{C}_{(13)}$ & 120.0 & $\mathrm{C}_{(4)}$ & $\mathrm{C}_{(5)}$ & $\mathrm{C}_{(6)}$ & $121.30(16)$ \\
\hline $\mathrm{C}_{(14)}$ & $\mathrm{C}_{(15)}$ & $\mathrm{C}_{(16)}$ & 120.0 & $\mathrm{C}_{(7)}$ & $\mathrm{C}_{(6)}$ & $\mathrm{C}_{(5)}$ & $120.98(18)$ \\
\hline $\mathrm{C}_{(15)}$ & $\mathrm{C}_{(16)}$ & $\mathrm{C}_{(11)}$ & 120.0 & $\mathrm{C}_{(1)}$ & $\mathrm{C}_{(17)}$ & $\mathrm{C}_{(19)}$ & $100.7(9)$ \\
\hline $\mathrm{N}_{(1)}$ & $\mathrm{C}_{(8)}$ & $\mathrm{C}_{(3)}$ & $107.64(12)$ & $\mathrm{C}_{(18)}$ & $\mathrm{C}_{(17)}$ & $\mathrm{C}_{(1)}$ & $100(2)$ \\
\hline $\mathrm{N}_{(1)}$ & $\mathrm{C}_{(8)}$ & $\mathrm{C}_{(7)}$ & $130.03(14)$ & $\mathrm{C}_{(18)}$ & $\mathrm{C}_{(17)}$ & $\mathrm{C}_{(19)}$ & 105 (3) \\
\hline $\mathrm{C}_{(7)}$ & $\mathrm{C}_{(8)}$ & $\mathrm{C}_{(3)}$ & $122.31(13)$ & $\mathrm{N}_{(2)}$ & $\mathrm{C}_{(18)}$ & $\mathrm{C}_{(17)}$ & $97(2)$ \\
\hline $\mathrm{C}_{(8)}$ & $\mathrm{C}_{(3)}$ & $\mathrm{C}_{(2)}$ & 106.35 ( 11$)$ & $\mathrm{C}_{(24)}$ & $\mathrm{C}_{(19)}$ & $\mathrm{C}_{(17)}$ & $94.6(12)$ \\
\hline $\mathrm{C}_{(4)}$ & $\mathrm{C}_{(3)}$ & $\mathrm{C}_{(8)}$ & $118.41(15)$ & $\mathrm{C}_{(24)}$ & $\mathrm{C}_{(19)}$ & $\mathrm{C}_{(20)}$ & 120.0 \\
\hline $\mathrm{C}_{(4)}$ & $\mathrm{C}_{(3)}$ & $\mathrm{C}_{(2)}$ & $135.24(15)$ & $\mathrm{C}_{(20)}$ & $\mathrm{C}_{(19)}$ & $\mathrm{C}_{(17)}$ & $144.7(12)$ \\
\hline $\mathrm{N}_{(1)}$ & $\mathrm{C}_{(1)}$ & $\mathrm{C}_{(2)}$ & $109.17(12)$ & $\mathrm{C}_{(23)}$ & $\mathrm{C}_{(24)}$ & $\mathrm{C}_{(19)}$ & 120.0 \\
\hline $\mathrm{N}_{(1)}$ & $\mathrm{C}_{(1)}$ & $\mathrm{C}_{(9)}$ & $117.65(13)$ & $\mathrm{C}_{(22)}$ & $\mathrm{C}_{(23)}$ & $\mathrm{C}_{(24)}$ & 120.0 \\
\hline $\mathrm{N}_{(1)}$ & $\mathrm{C}_{(1)}$ & $\mathrm{C}_{(17)}$ & $142.3(8)$ & $\mathrm{C}_{(23)}$ & $\mathrm{C}_{(22)}$ & $\mathrm{C}_{(21)}$ & 120.0 \\
\hline $\mathrm{C}_{(2)}$ & $\mathrm{C}_{(1)}$ & $\mathrm{C}_{(9)}$ & $133.17(15)$ & $\mathrm{C}_{(20)}$ & $\mathrm{C}_{(21)}$ & $\mathrm{C}_{(22)}$ & 120.0 \\
\hline $\mathrm{C}_{(2)}$ & $\mathrm{C}_{(1)}$ & $\mathrm{C}_{(17)}$ & $106.0(10)$ & $\mathrm{C}_{(21)}$ & $\mathrm{C}_{(20)}$ & $\mathrm{C}_{(19)}$ & 120.0 \\
\hline $\mathrm{C}_{(1)}$ & $\mathrm{C}_{(2)}$ & $\mathrm{C}_{(3)}$ & $107.31(13)$ & & & & \\
\hline
\end{tabular}


Table S6. Hydrogen Atom Coordinates $\left(\AA \times 10^{4}\right)$ and Isotropic Displacement Parameters $\left(\AA^{2} \times 10^{3}\right)$ for ANNA1229_tryptamine_2.

$\begin{array}{lr}\text { Atom } & \boldsymbol{x} \\ \mathrm{H}_{(12)} & 5598.86 \\ \mathrm{H}_{(13)} & 5389.65 \\ \mathrm{H}_{(14)} & 5566.98 \\ \mathrm{H}_{(15)} & 5953.52 \\ \mathrm{H}_{(16)} & 6162.74 \\ \mathrm{H}_{(2)} & 6609.59 \\ \mathrm{H}_{(9)} & 5662.58 \\ \mathrm{H}_{(7)} & 2153.01 \\ \mathrm{H}_{(4)} & 5312.1 \\ \mathrm{H}_{(10 A)} & 7328.48 \\ \mathrm{H}_{(10 B)} & 7691.18 \\ \mathrm{H}_{(5)} & 3467.61 \\ \mathrm{H}_{(6)} & 1907.89 \\ \mathrm{H}_{(17)} & 7236.02 \\ \mathrm{H}_{(18 \mathrm{~A})} & 6852.69 \\ \mathrm{H}_{(18 \mathrm{~B})} & 7216.21 \\ \mathrm{H}_{(2 \mathrm{~A})} & 5042.64 \\ \mathrm{H}_{(2 \mathrm{~B})} & 5312.83 \\ \mathrm{H}_{(1)} & 3852(15) \\ \mathrm{H}_{(24)} & 6685.94 \\ \mathrm{H}_{(23)} & 6470.55 \\ \mathrm{H}_{(22)} & 5615.67 \\ \mathrm{H}_{(21)} & 4976.18 \\ \mathrm{H}_{(20)} & 5191.56 \\ \mathrm{H}_{(3 \mathrm{~A})} & 8090(20) \\ \mathrm{H}_{(3 \mathrm{~B})} & 7480(20) \\ & \end{array}$

$\begin{array}{rr}\boldsymbol{z} & \mathbf{U}(\mathbf{e q}) \\ 7300.82 & 64 \\ 6623.27 & 74 \\ 5430.61 & 86 \\ 4915.51 & 88 \\ 5593.05 & 67 \\ 6347.39 & 75 \\ 7414.91 & 61 \\ 6294.8 & 91 \\ 5766.53 & 95 \\ 7381.88 & 82 \\ 6688.87 & 82 \\ 5518.75 & 111 \\ 5765.74 & 108 \\ 6479.32 & 100 \\ 7668.77 & 153 \\ 7614.39 & 153 \\ 7281.91 & 70 \\ 8034.92 & 70 \\ 6836(10) & 84(5) \\ 5656.34 & 71 \\ 4928.87 & 91 \\ 5336.42 & 110 \\ 6471.45 & 68 \\ 7198.93 & 57 \\ 7252(12) & 103(9) \\ 7783(12) & 85(8)\end{array}$


Table S7. Atomic Occupancy for ANNA1229_tryptamine_2.

\begin{tabular}{lcllll} 
Atom & Occupancy & \multicolumn{1}{c}{ Atom } & Occupancy & Atom $^{\text {Occupancy }}$ \\
$\mathrm{C}_{(11)}$ & $0.51(3)$ & $\mathrm{C}_{(12)}$ & $0.51(3)$ & $\mathrm{H}_{(12)}$ & $0.51(3)$ \\
$\mathrm{C}_{(13)}$ & $0.51(3)$ & $\mathrm{H}_{(13)}$ & $0.51(3)$ & $\mathrm{C}_{(14)}$ & $0.51(3)$ \\
$\mathrm{H}_{(14)}$ & $0.51(3)$ & $\mathrm{C}_{(15)}$ & $0.51(3)$ & $\mathrm{H}_{(15)}$ & $0.51(3)$ \\
$\mathrm{C}_{(16)}$ & $0.51(3)$ & $\mathrm{H}_{(16)}$ & $0.51(3)$ & $\mathrm{N}_{(3)}$ & $0.900(4)$ \\
$\mathrm{C}_{(9)}$ & $0.900(4)$ & $\mathrm{H}_{(9)}$ & $0.900(4)$ & $\mathrm{C}_{(10)}$ & $0.900(4)$ \\
$\mathrm{H}_{(10 \mathrm{~A})}$ & $0.900(4)$ & $\mathrm{H}_{(10 \mathrm{~B})}$ & $0.900(4)$ & $\mathrm{C}_{(17)}$ & $0.100(4)$ \\
$\mathrm{H}_{(17)}$ & $0.100(4)$ & $\mathrm{C}_{(18)}$ & $0.100(4)$ & $\mathrm{H}_{(18 \mathrm{~A})}$ & $0.100(4)$ \\
$\mathrm{H}_{(18 \mathrm{~B})}$ & $0.100(4)$ & $\mathrm{N}_{(2)}$ & $0.100(4)$ & $\mathrm{H}_{(2 \mathrm{~A})}$ & $0.09(3)$ \\
$\mathrm{H}_{(2 \mathrm{~B})}$ & 0.1 & $\mathrm{C}_{(19)}$ & $0.49(3)$ & $\mathrm{C}_{(24)}$ & $0.49(3)$ \\
$\mathrm{H}_{(24)}$ & $0.49(3)$ & $\mathrm{C}_{(23)}$ & $0.49(3)$ & $\mathrm{H}_{(23)}$ & $0.49(3)$ \\
$\mathrm{C}_{(22)}$ & $0.49(3)$ & $\mathrm{H}_{(22)}$ & $0.49(3)$ & $\mathrm{C}_{(21)}$ & $0.49(3)$ \\
$\mathrm{H}_{(21)}$ & $0.49(3)$ & $\mathrm{C}_{(20)}$ & $0.49(3)$ & $\mathrm{H}_{(20)}$ & $0.49(3)$ \\
$\mathrm{H}_{(3 \mathrm{~A})}$ & $0.91(3)$ & $\mathrm{H}_{(3 \mathrm{~B})}$ & 0.9 & &
\end{tabular}




\section{Experimental}

Single crystals of $\mathrm{C}_{16} \mathrm{H}_{16} \mathrm{~N}_{2}$ (38) [ANNA1229_tryptamine_2] were prepared by slow evaporation of chloroform solution. A suitable crystal was selected and mounted by acrylic glue on the glass stick on a SuperNova, Dual, Cu at home/near, AtlasS2 diffractometer. The crystal was kept at 293(2) K during data collection. Using Olex $2,{ }^{\mathrm{S} 1}$ the structure was solved with the ShelXT ${ }^{\mathrm{S} 2}$ structure solution program using Intrinsic Phasing and refined with the ShelXL ${ }^{\mathrm{S} 3}$ refinement package using Least Squares minimization.

\section{Crystal structure determination of [ANNA1229_tryptamine_2]}

Crystal Data for $\mathrm{C}_{16} \mathrm{H}_{16} \mathrm{~N}_{2}(M=236.31 \mathrm{~g} / \mathrm{mol})$ : orthorhombic, space group Pbca (no. 61), $a=11.62377(13) \AA, b=11.62379(12) \AA, c=19.3359(2) \AA, V=$ $2612.52(5) \AA^{3}, Z=8, T=293(2) \mathrm{K}, \mu(\mathrm{CuK \alpha})=0.550 \mathrm{~mm}^{-1}$, Dcalc $=1.202 \mathrm{~g} / \mathrm{cm}^{3}, 31528$ reflections measured $\left(9.148^{\circ} \leq 2 \Theta \leq 152.952^{\circ}\right), 2722$ unique $(R$ int $=$ 0.0157 , $\left.\mathrm{R}_{\text {sigma }}=0.0057\right)$ which were used in all calculations. The final $R_{1}$ was $0.0430(\mathrm{I}>2 \sigma(\mathrm{I}))$ and $w R_{2}$ was 0.1348 (all data).

\section{Refinement model description}

\section{Details:}

Number of restraints - 0 , number of constraints - unknown.

1. Fixed Uiso

At 1.2 times of:

All C $(\mathrm{H})$ groups, All $\mathrm{C}(\mathrm{H}, \mathrm{H})$ groups, $\mathrm{All} \mathrm{N}(\mathrm{H}, \mathrm{H})$ groups

2. Others

Sof $(\mathrm{C} 17)=\operatorname{Sof}(\mathrm{H} 17)=\operatorname{Sof}(\mathrm{C} 18)=\operatorname{Sof}(\mathrm{H} 18 \mathrm{~A})=\operatorname{Sof}(\mathrm{H} 18 \mathrm{~B})=\operatorname{Sof}(\mathrm{N} 2)=1-\mathrm{FVAR}(1)$

Sof $(\mathrm{N} 3)=\operatorname{Sof}(\mathrm{C} 9)=\operatorname{Sof}(\mathrm{H} 9)=\operatorname{Sof}(\mathrm{C} 10)=\operatorname{Sof}(\mathrm{H} 10 \mathrm{~A})=\operatorname{Sof}(\mathrm{H} 10 \mathrm{~B})=\mathrm{FVAR}(1)$

Sof $(\mathrm{C} 19)=\operatorname{Sof}(\mathrm{C} 24)=\operatorname{Sof}(H 24)=\operatorname{Sof}(\mathrm{C} 23)=\operatorname{Sof}(\mathrm{H} 23)=\operatorname{Sof}(\mathrm{C} 22)=\operatorname{Sof}(\mathrm{H} 22)=\operatorname{Sof}(\mathrm{C} 21)=$

Sof $($ H21) $=$ Sof $($ C20 $)=$ Sof $($ H2O $)=1-F V A R(2)$

Sof $(\mathrm{Cl1})=\operatorname{Sof}(\mathrm{C12})=\operatorname{Sof}($ H12 $)=\operatorname{Sof}(\mathrm{C13})=\operatorname{Sof}(\mathrm{H13})=\operatorname{Sof}(\mathrm{C} 14)=\operatorname{Sof}(H 14)=\operatorname{Sof}(\mathrm{C} 15)=$

Sof $($ H15) $=$ Sof $(\mathrm{C1} 6)=\operatorname{Sof}($ H16) $=$ FVAR $(2)$

Sof $($ H3A $)=1-$ FVAR (3)

SOf $($ H2A $)=$ FVAR $(3)$

Fixed Sof: H2B (0.1) H3B (0.9)

3. a Ternary $\mathrm{CH}$ refined with riding coordinates:

C9 (H9), C17 (H17)

3.b Secondary $\mathrm{CH} 2$ refined with riding coordinates:

$\mathrm{C} 10$ (H10A, H10B), C18 (H18A, H18B)

3.C Aromatic/amide $\mathrm{H}$ refined with riding coordinates:

$\mathrm{C} 12$ (H12), C13(H13), C14(H14), C15(H15), C16(H16), C2(H2), C7(H7), C4(H4),

C5(H5), C6(H6), C24(H24), C23(H23), C22(H22), C21(H21), C2O(H2O)

3.d Fitted hexagon refined as free rotating group:

$\mathrm{C} 11(\mathrm{C} 12, \mathrm{C} 13, \mathrm{C} 14, \mathrm{C} 15, \mathrm{C} 16), \mathrm{C} 19(\mathrm{C} 24, \mathrm{C} 23, \mathrm{C} 22, \mathrm{C} 21, \mathrm{C} 20)$

3.e $\mathrm{X}=\mathrm{CH} 2$ refined with riding coordinates:

$\mathrm{N} 2(\mathrm{H} 2 \mathrm{~A}, \mathrm{H} 2 \mathrm{~B})$ 


\section{References Cited}

(S1) Dolomanov, O.V., Bourhis, L.J., Gildea, R.J, Howard, J.A.K. \& Puschmann, H. OLEX2: a Complete Structure Solution, Refinement and Analysis Program. J. Appl. Cryst. 2009, 42, 339-341.

(S2) Sheldrick, G.M. SHELXT - Integrated Space-Group and Crystal-Structure Determination. Acta Cryst. 2015, A71, 3-8.

(S3) Sheldrick, G.M. Crystal Structure Refinement with SHELXL. Acta Cryst. 2015, C71, 3-8. 